\title{
Theoretical Nanoscale Design of Self-Cleaning Coatings
}

\author{
A Thesis Submitted \\ in Fulfillment of the \\ Requirements for the degree of \\ DOCTOR OF PHILOSOPHY
}

George Yiapanis

B. App. Sci. Hons.

\author{
Applied Physics \\ School of Applied Sciences \\ College of Science, Engineering and Health \\ RMIT University \\ April, 2010
}




\section{DECLARATION OF CANDIDATURE}

I certify that except where due acknowledgment has been made, that the work is that of the candidate alone. This body of work has not been submitted previously, in whole or in part, to qualify for any other academic award. The content of this thesis is the result of work which as been carried out since the official commencement date of the approved research program. Any editorial work, paid or unpaid, carried out by a third party is acknowledged.

George Yiapanis 


\section{CONTENTS}

DECLARATION OF CANDIDATURE .................................................................... ii

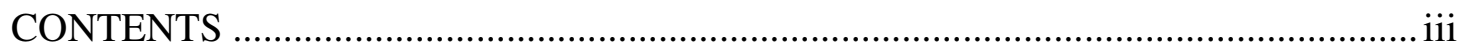

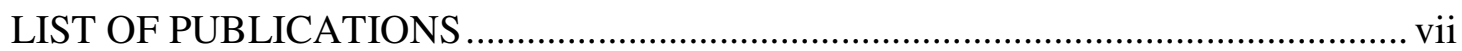

LIST OF CONFERENCE PRESENTATIONS ......................................................... viii

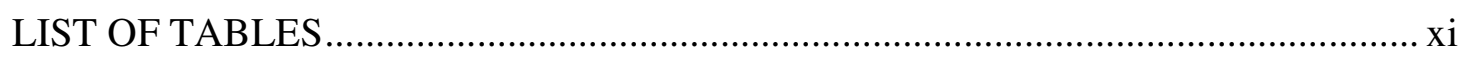

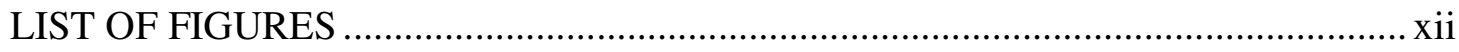

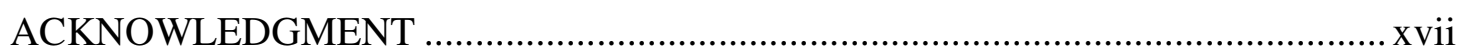

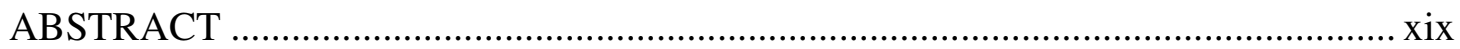

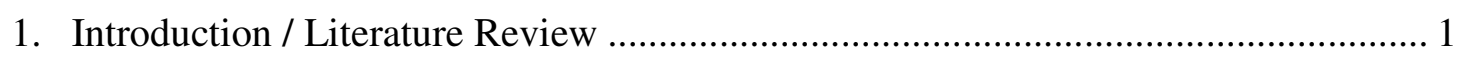

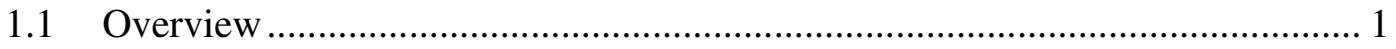

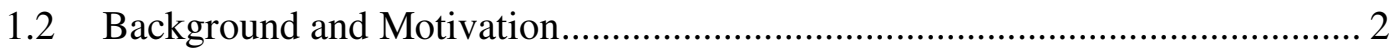

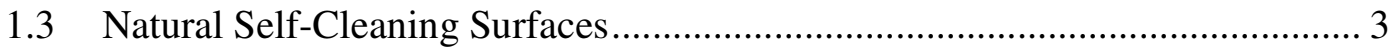

1.4 Parameters Affecting Adhesion and Wettability.......................................... 5

1.5 Modelling Studies of Polymer Surfaces and Interfaces ................................. 14

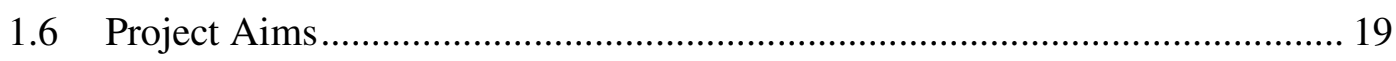

2. Principles of Atomistic Simulations of Large Systems ........................................... 20

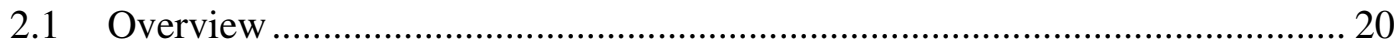

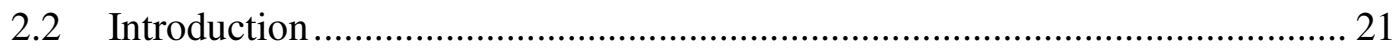

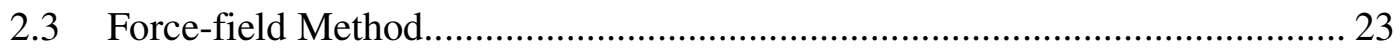

2.3.1 Force-field Energy Expression.............................................................. 23

2.3.2 Force-Field Parameterization ........................................................ 28

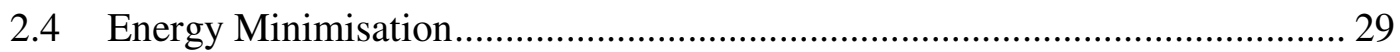

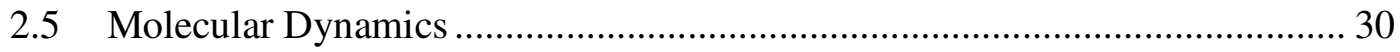

2.6 Periodic Boundary Conditions …............................................................... 32

2.7 Truncation of Non-Bonded Interactions ..................................................... 32

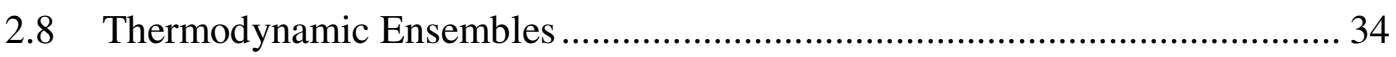




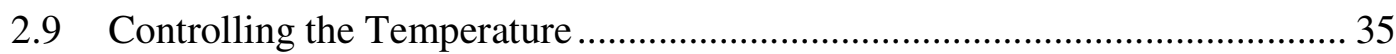

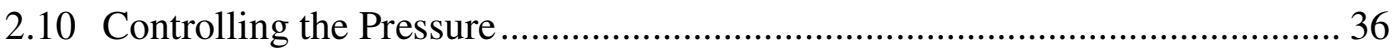

3. Molecular Models and Theoretical Procedures ......................................................... 37

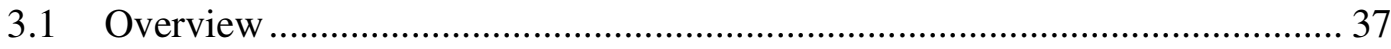

3.2 Polyester Surface Models............................................................................ 38

3.3 Models of Carbon Contaminants ............................................................... 40

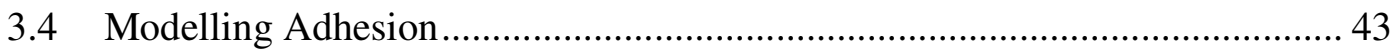

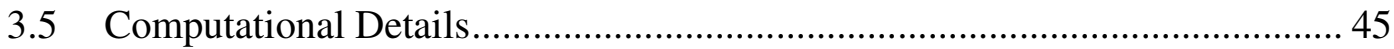

4. Effects of Surface Composition and Atomic Roughness on Adhesion ..................... 47

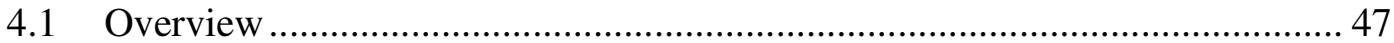

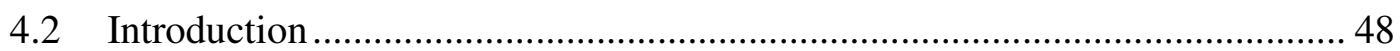

4.3 Molecular Models and Theoretical Procedures............................................. 50

4.3.1 Composition of Molecular Models ........................................................ 50

4.3.2 Theoretical Procedures.................................................................. 51

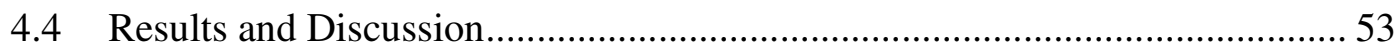

4.4.1 Rigid Interfaces $\left(\right.$ Systems $\mathrm{Nb}^{\text {rig }}$ ) ……............................................ 53

4.4.2 Energy Minimised Interfaces (Systems $N b^{\text {min }}$ ) ............................... 60

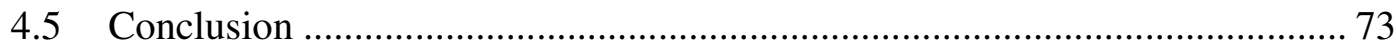

5. Effects of Aging on Adhesion between Polyester and Carbon................................. 75

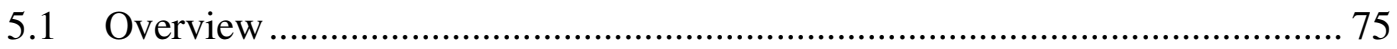

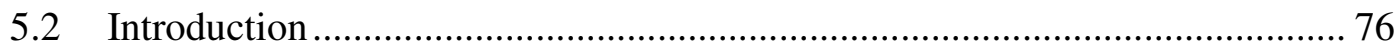

5.3 Molecular Models and Theoretical Procedures.............................................. 78

5.3.1 Composition of Molecular Models .................................................. 78

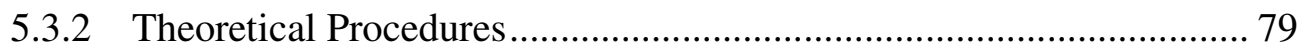

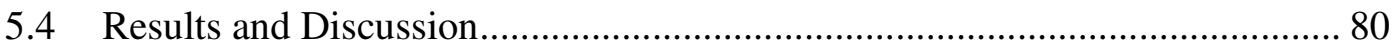

5.4.1 Polyester and Graphite Interfaces ...................................................... 80

5.4.2 Polyester and Amorphous Carbon Interfaces..................................... 83 
5.4.3 Discussion 86

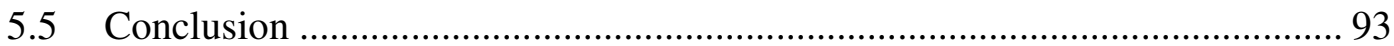

6. Polyester Surfaces and Fullerene in Aqueous Environment..................................... 94

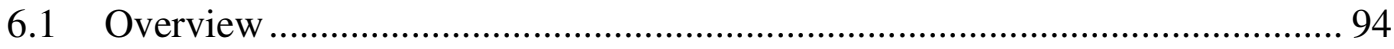

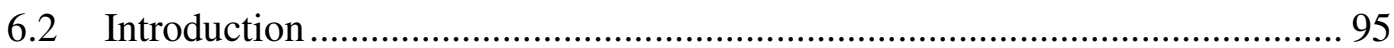

6.3 Molecular Models and Theoretical Procedures............................................... 96

6.3.1 Composition of Molecular Models ....................................................... 96

6.3.2 Theoretical Procedures................................................................ 100

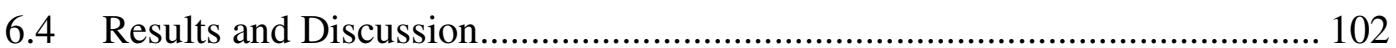

6.4.1 The Polyester and Water Interface ................................................... 102

6.4.2 Adhesion of $\mathrm{C}_{60}$ on Hydrated Unmodified Polyester......................... 108

6.4.3 Adhesion of $\mathrm{C}_{60}$ on Hydrated Modified Polyester ............................. 113

6.4.4 Effects of Water Layer Thickness on Polyester- $\mathrm{C}_{60}$ Adhesion........... 118

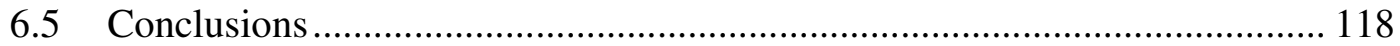

7. Effects of Surface Crosslinking on Adhesion and Hardness .................................. 119

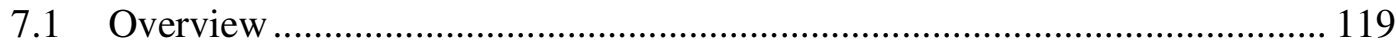

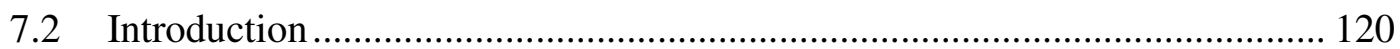

7.3 Molecular Models and Theoretical Procedures............................................ 122

7.3.1 Composition of Molecular Models ….............................................. 122

7.3.2 Modelling Surface Crosslinking of Polymers ................................... 124

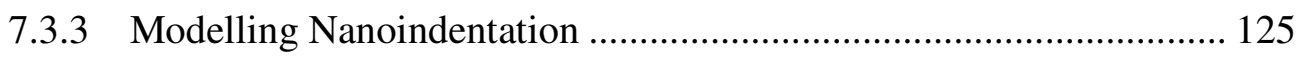

7.3.4 Computational Details................................................................ 128

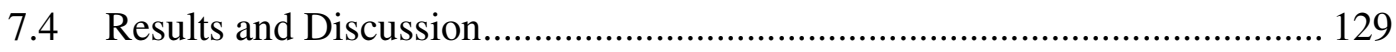

7.4.1 Surface Crosslinking of Polyester films........................................... 129

7.4.2 Force plots of polyester- $\mathrm{C}_{60}$ systems............................................. 132

7.4.3 Adhesion plots of polyester- $\mathrm{C}_{60}$ systems ...................................... 138

7.4.4 Hardness of polyester surfaces ....................................................... 141 


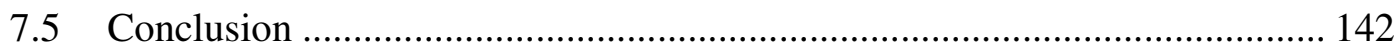

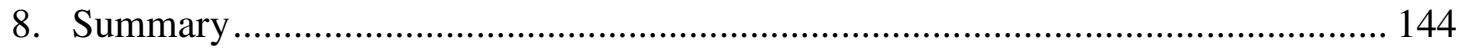

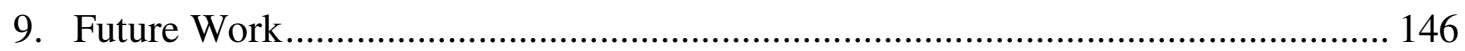

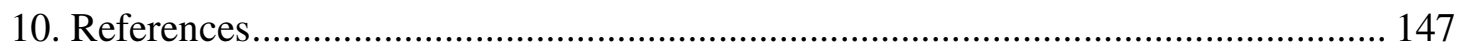




\section{LIST OF PUBLICATIONS}

YAROVSKY, I., HENRY, D. J., YIAPANIS, G. \& EVANS, E. (2006) Colorbond ${ }^{\circledR}$ meets nanotech: understanding coating interactions with the environment. Chemistry in Australia, 73, 11-13.

YIAPANIS, G., HENRY, D. J., EVANS, E. \& YAROVSKY, I. (2007)

Effect of Surface Composition and Atomic Roughness on Interfacial Adhesion between Polyester and Amorphous Carbon. J. Phys. Chem. C, 111, 3000-3009.

YIAPANIS, G., HENRY, D. J., EVANS, E. \& YAROVSKY, I. (2007) Effect of Aging on Interfacial Adhesion between Polyester and Carbon-Based Particles: A Classical Molecular Dynamics Study. J. Phys. Chem. C, 111, 6456-6472.

YIAPANIS, G., HENRY, D. J., EVANS, E. \& YAROVSKY, I. (2008) Molecular Dynamics Study of Polyester Surfaces and Fullerene Particles in Aqueous Environment. J. Phys. Chem. C, 112, 18141-18149.

YIAPANIS, G., EVANS, E., HENRY, D. J. \& YAROVSKY, I. (2010) Simulations of Nanoindentation of Polymer Surfaces: Effects of Surface Cross-Linking on Adhesion and Hardness. J. Phys. Chem. C, 114, 478-486.

YIAPANIS, G., HENRY, D. J., EVANS, E. \& YAROVSKY, I. (to appear 2011) Theoretical Nanoscale Design of Self-Cleaning Coatings in "Nanotechnology in Australia: Showcase of Early Career Research in Australia" edited by Kane, D. M., Micolich, A. P. and Rabeau, J. R. Pan Stanford Publishing. 


\section{LIST OF CONFERENCE PRESENTATIONS}

"YIAPANIS, G., HENRY, D. J., EVANS, E. \& YAROVSKY, I.

Simulations of Nanoindentation of Polymer Surfaces: Effects of Surface Crosslinking on Adhesion and Hardness. The $12^{\text {th }}$ Australian Molecular Modelling Conference, Gold Coast, 2009.

*YAROVSKY, I., YIAPANIS, G., HENRY, D. J. \& EVANS, E.

Nanoscale Design of Contamination Resistant Polymer Surfaces. International Symposium on Recent Development and Applications in Polymer Nanostructured Materials, Melbourne, 2009.

*YIAPANIS, G., HENRY, D. J., EVANS, E. \& YAROVSKY, I.

Fullerene Interactions with Polyester Surfaces in Water. The 30th Australasian Polymer Symposium, Melbourne, 2009.

*YAROVSKY, I., YIAPANIS, G., HENRY, D. J. \& EVANS, E.

Theoretical Nanoscale Design of Contamination Resistant Surfaces. WATOC, Sydney, 2008.

*YAROVSKY, I., YIAPANIS, G., HENRY, D. J. \& EVANS, E.

Theoretical Design of New Materials. Queen Mary University, London, 2008 (Invited Lecture).

*YAROVSKY, I., YIAPANIS, G., HENRY, D. J. \& EVANS, E.

Self Cleaning Surfaces: Design Principles from Simulation. Imperial College London, Thomas Young Centre for Materials Modelling and Simulation, 2008 (Invited Lecture).

${ }^{*}$ Presenter 
*YAROVSKY, I., YIAPANIS, G., HENRY, D. J. \& EVANS, E.

Theoretical Nanoscale Design of Novel Materials. Australian Institute for Bioengineering and Nanotechnology, University of Queensland, 2008 (Invited Lecture).

*HENRY, D. J., YIAPANIS, G., EVANS, E. \& YAROVSKY, I.

Nanocarbon Interaction with Coatings: An Insight from Molecular Dynamics Studies of Fullerene Interaction with Silica and Polyester. Organic and Physical Chemistry (OPC) Conference, Adelaide, 2007.

*YIAPANIS, G., HENRY, D. J., EVANS, E. \& YAROVSKY, I.

MD Study of Fullerene Interactions with Polyester Surfaces in Water. The $11^{\text {th }}$ Australian Molecular Modelling Conference, Melbourne, 2007 (Poster Presentation).

\section{Awarded Poster Prize.}

*YIAPANIS, G., HENRY, D. J., EVANS, E. \& YAROVSKY, I.

Design Principles for Clean Polyester Coatings. Workshop: Interaction Energies and the Structure of Surfaces and Nano-Structures, RMIT, Melbourne, 2007.

*YAROVSKY, I., YIAPANIS, G., HENRY, D. J. \& EVANS, E.

Theoretical Nanoscale Design of Self Cleaning Coatings. Professorial Fellowship, Japanese Government Centre of Excellence for Promotion of Science, Kyoto University, 2007 (Invited Lecture).

*YIAPANIS, G., EVANS, E., HENRY, D. J. \& YAROVSKY, I.

The Effect of Composition and Surface Roughness on Interfacial Adhesion between Polyester and Amorphous Carbon. The $10^{\text {th }}$ Australian Molecular Modelling Conference, Perth, 2006.

Awarded Student's Bursary.

${ }^{*}$ Presenter 
"YAROVSKY, I., HENRY, D. J., YIAPANIS, G. \& EVANS, E.

Atomistic Simulation of Interactions of Carbon Nano-particles with Organic Coatings. International Conference "Trends in Nanotechnology", France, 2006.

*YAROVSKY, I., HENRY, D. J., YIAPANIS, G. \& EVANS, E.

Nanoscale Design of Contamination Resistant Coatings, International Forum NanoEurope, St Gallen, Switzerland, 2006.

${ }^{*}$ Presenter 


\section{LIST OF TABLES}

Table 1.1 Surface Free Energy of Common Polymers Measured at $20^{\circ} \mathrm{C}$. ....................... 6

Table 3.1 Polyester Surfaces Examined ....................................................................... 40

Table 4.1 Adhesion Parameters for Rigid Interfaces (Systems $\mathrm{Nb}^{\mathrm{rig}}$ ) ........................... 53

Table 4.2 Adhesion Parameters for Energy Minimised Interfaces (Systems $\mathrm{Nb}^{\mathrm{min}}$ ) ....... 61

Table 5.1 Adhesion Parameters for Polyester/Graphite Interfaces (Systems Na)........... 81

Table 5.2 Adhesion Parameters for Polyester/Amorphous Carbon Interfaces

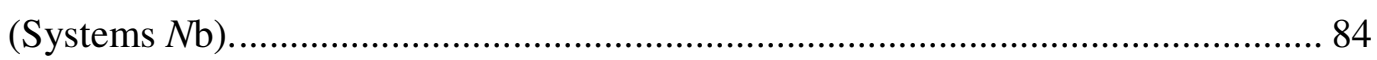

Table 6.1 Characteristic Features of Specific Regions of the Polyester Surfaces ........... 98

Table 6.2 Average Equilibrium Separation Values $\left(d_{a v}\right)$ between $\mathrm{C}_{60}$ and Hydrated

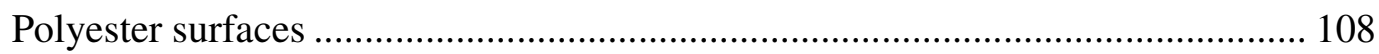

Table 7.1 Hardness Parameters for Polyester Surfaces ............................................... 141 


\section{LIST OF FIGURES}

Figure 1.1 Schematic illustration of self-cleaning surfaces. a) The Lotus effect combines natural hydrophobicity with a complex surface structure to ensure water droplets easily roll-off the surface carrying with them unwanted particulates. b) On a super-hydrophilic self-cleaning surface, water droplets

spread forming a thin film of water, lifting contaminants from the surface.

Figure 1.2 Illustration of a liquid drop on a solid surface forming a contact angle $\theta$. According to the Young-Dupré equation (eq. (1.1)), the contact angle is related to the surface and interfacial free energies $(\gamma)$ of the comprising media...... 5

Figure 1.3 Schematic illustrations of the Wenzel and Cassie-Baxter constructions, which determine the influence of roughness on the wettability of the surface.

Figure 2.1 Lennard Jones 9-6 potential for two isolated carbon atoms, comprising of a repulsive component, varying as the inverse ninth power of the interatomic distance, and an attractive component, varying as the inverse twelfth power of the distance

Figure 3.1 Chemical composition of the monomeric units used to construct the polyester coating model

Figure 3.2 Model of a nano-scale modified surface coating. Surface modifications are undertaken by replacement of hydrogen atoms, with fluorine (F), hydroxyl $(\mathrm{OH})$ or carboxyl $\left(\mathrm{CO}_{2} \mathrm{H}\right)$ groups. In this figure, the hydrogen, fluorine, oxygen and carbon atoms are represented by white, light blue, red and grey beads respectively. The fluorine modifications are employed to improve the carbon-shielding ability of the coating

Figure 3.3 Contaminant models. a, Graphite represents the surface of an ideal soot particle. $b$, The Amorphous Carbon $(\mathrm{AmCH})$ model is based on realistic coal derived contaminant and exhibits an irregular surface morphology analogous to tar-balls. c, The fullerene $\mathrm{C}_{60}$ model represents a soot particulate.

Figure 3.4 A schematic representation of the polyester/amorphous carbon interface, and the interfacial separation $d$. This interface represents a carbon contaminant interacting with a polyester surface coating. The interfacial separation is the distance between the boundaries of the polymer and carbon 
models. The boundaries were determined by averaging the $\mathrm{z}$ positions of atoms in the leading $2.5 \AA$ of the materials.

Figure 3.5 Schematic plot of the adhesion energy curve with the work of separation $\left(W_{\text {sep }}\right)$, equilibrium interfacial separation $\left(d_{0}\right)$ and interaction range $(l)$. The work of separation was evaluated by the depth of the potential well of the curve and is a measure of the strength of adhesion at the interface. The equilibrium separation $d_{0}$, is a measure of the typical spacing between surfaces, while the interaction range represents the range over which the two surfaces interact.

Figure 3.6 Conceptual illustration of the Contact Volume, $V_{c}$. It provides a measure of the effective contact between interacting surfaces.

Figure 4.1 Adhesion energy plots of modified polyesters interacting with amorphous carbon $(\mathrm{AmCH})$. The unmodified interface, polyester/ $\mathrm{AmCH}$, is also included as a reference point. The depth of the well in these curves provides a measure of the adhesion at the interface, and the greater the dip, the stronger the adhesion between coating (polymer) and contaminant $(\mathrm{AmCH})$. Adhesion plots show that low-level hydroxyl $(7 \mathrm{OH})$ surface modifications reduce adhesion between coating and contaminant.

Figure 4.2 Plots of the vdW energy between atoms as a function of their interatomic separation. These plots compare the $\mathrm{vdW}$ interactions between polymer surface atoms (hydrogen, fluorine, carbon and oxygen) with aromatic carbons of graphite (C'). 'Heavy' atoms such as F, C and O offer a relatively strong repulsion with aromatic carbon at short interatomic separations, while hydrogen $\left(\mathrm{H}\right.$ or $\left.\mathrm{H}^{*}\right)$ offers a relatively weak repulsion.

Figure 4.3 Adhesion energy plots for polymer/amorphous carbon interfaces highlighting the importance of rigidity as a preventative measure for surface contamination. The amorphous carbon surface represents a carbon contaminant, and the polyesters represent various paint coatings. The depth of the well in these plots provide a measure of the adhesion at the interface, and the greater the dip, the stronger the adhesion between coating and contaminant. The amorphous carbon contaminant is seen to adhere 
considerably weaker to the rigid and atomically rough polyester than the energy minimised polymers.

Figure 4.4 Schematic representation of the effects of relaxation on polyester/carbon interfaces. Relaxation against the graphite structure, results in the smoothing of the polymer coating. Relaxation against the amorphous carbon model, leads to interfacial interlocking. In both of these examples, structural irregularities at the interface are effectively eliminated.

Figure 4.5 Scale representation of the average separation between amorphous carbon and the polyester substituents, and the equilibrium separation, for rigid and energy minimised systems.

Figure 4.6 Snapshot of energy minimised Polyester75OH/AmCH interface, highlighting the possible hydrogen bonding regions and with the inclusion of donor-acceptor distances in $\AA$.

Figure 5.1 Snapshots of the polyester/graphite interface highlighting the repositioning of phenyl rings as a result of MD.

Figure 5.2 Concentration profile of carbon atoms in phenyl rings for various polyester models, as a function of the average separation from the graphite and amorphous carbon boundaries.

Figure 5.3 Scale representation of the average separation between carbon and polyester substituents, as well as the equilibrium separation for the relaxed interfaces; Polyester/graphite (Systems $\mathrm{Na}^{\mathrm{rel}}$ ) and Polyester/AmCH (Systems $N \mathrm{~b}^{\mathrm{rel}}$ ).

Figure 6.1 Contoured vdW slice of polyester model taken along the surface boundary in its initial configuration.

Figure 6.2 Schematic representation of the $\mathrm{C}_{60}$ particle interacting with a hydrated polyester surface, polyester/ $\mathrm{H}_{2} \mathrm{O} / \mathrm{C}_{60}$. The carbon nano-particle represents a soot particulate and the hydrated polymer film represents a wetted surface coating......

Figure 6.3 Concentration profiles of polyester $/ \mathrm{H}_{2} \mathrm{O}$ systems. Profiles of the polymer surfaces are displayed to the left-hand side of the graphs, while the water profiles are displayed toward the right-hand side. 
Figure 6.4 Radial distribution functions $\mathrm{g}(\mathrm{r})$ of surface atoms in polyester (indicated by a prime), with $\mathrm{O}$ and $\mathrm{H}$ atoms of water. Values of $\mathrm{g}(\mathrm{r})$ are normalized to the overall density (including vacuum space) of the unit cell.

Figure 6.5 Variation in the total number of hydrogen bonds per water molecule with average z position. Cut-off hydrogen bond distance of $2.5 \AA$ and cut-off hydrogen bond angle of $90^{\circ}$ were used as a criteria for H-bond calculation. ${ }^{194}$

Figure 6.6 Number of water molecules as a function of radial distance from the nearest polymer atom.

Figure 6.7 Snaphots of polyester/water systems highlighting hydrogen bonding configurations. For an improve clarity of the image, selected atoms have been omitted.

Figure 6.8 Approach curve for a typical Polyester/ $\mathrm{H}_{2} \mathrm{O} / \mathrm{C} 60$ system. Insets show snapshots of initial and final configurations of the system.

Figure 6.9 Selected radial distribution functions of carbon in polyester $\left(C^{\prime}\right)$ and carbon of $\mathrm{C}_{60}(\mathrm{C})$. These radial distribution functions provide an indication of the extent of interaction between the fullerene and hydrophobic segments of the polyester.

Figure 6.10 A close-up snapshot of fullerene particle inside a polyester layer. The image has been captured during molecular dynamics. It is representative of a typical equilibrium state and highlights a common cage-like formation between hydrophobic segments of the interface.

Figure 6.11 Selected radial distribution functions of carbon in polyester $\left(C^{\prime}\right)$ with carbon of $\mathrm{C}_{60}(\mathrm{C})$. These radial distribution functions provide an indication of the extent of interaction between the fullerene and hydrophobic segments of the polyester.

Figure 6.12 A cross-sectional slice taken through the vdW surface of the systems. The polyester surface is indicated in red, the water layer is indicated in blue and the fullerene particle is represented by the yellow, ball and stick structure. Areas of low color intensity along the slice, correspond to regions of reduced atomic concentration while more intense colors, represent regions of increased atomic concentration. (a) In the absence of surface modifications, 
the fullerene particle is seen to infiltrate the polymer layer. (b) In the case of the hydroxyl modified polymer, water situated underneath the $\mathrm{C}_{60}$ particle, reduces the extent of contact between $\mathrm{C}_{60}$ and the substrate.

Figure 7.1 Chemical composition of the di-isocyanate crosslinking molecules and the chemical formula of the related crosslinking reaction where $\mathrm{R}=\left(\mathrm{CH}_{2}\right)_{6}$ and $\mathrm{R}=\mathrm{C}_{10} \mathrm{H}_{8}$ for Hexamethylene di-isocyanate (HDI) and Isophorone diisocyanate (IPDI) crosslinkers respectively.....

Figure 7.2 Schematic diagram showing indentation of a polyester layer using a fullerene $\mathrm{C}_{60}$ particle. The graphene sheet mimics a rigid substrate and provides support during indentation.

Figure 7.3 (a) Percentage of reacted $\mathrm{OH}$ groups during crosslinking (b) Percentage of crosslinks formed during crosslinking (c) Percentage of dangling residues during crosslinking.

Figure 7.4 Plots of the normal force on the $\mathrm{C}_{60}$ particle versus the average distance between $\mathrm{C}_{60}$ and surface.

Figure 7.5 Overlayed snapshots of vdW contours of the unmodified polyester surface and $\mathrm{C}_{60}$ particle during indentation.

Figure 7.6 Overlayed snapshots of vdW contours of the Polyester15HDI surface and $\mathrm{C}_{60}$ particle during indentation

Figure 7.7 Snapshots of the equilibrated structures during loading highlighting two common forms of interfacial re-structuring: (a) conformational change of hexamethylene di-isocyanate (b) displacement of isophorone di-isocyanate........ 136

Figure 7.8 Overlayed snapshots of vdW contours of the Polyester14IPDI surface and $\mathrm{C}_{60}$ particle during indentation.

Figure 7.9 Adhesion energy plots for polyester- $\mathrm{C}_{60}$ systems 138 


\section{ACKNOWLEDGMENT}

This work would not have been made possible without the support, guidance, and collaboration of many people who deserve to be acknowledged.

Firstly, I would like to acknowledge my supervisor, Prof. Irene Yarovsky. Thank you for giving me the opportunity to work under your guidance. Thank you for all your support, patience, and understanding. Your drive and depth of knowledge have proved invaluable to this project, and you have made this experience possible, enjoyable and memorable.

I would also like to extend a very special thank you to my second supervisor, Dr. David Henry. I sincerely thank you for all your help and your contribution to this work. You're vast scientific knowledge never ceases to amaze me. For the duration of this project, you have answered all my questions, and I have learnt a great deal working under your guidance.

I would like to thank Dr. Evan Evans from BlueScope Steel Laboratories, Wollongong, for being one of the driving forces behind this project. Your ideas, suggestions and experimental support have greatly improved this project.

I would like to thank the Australia Research Council and Bluescope Steel for providing financial support for this project.

I would also like to acknowledge the National Computing Infrastructure (NCI) and the Victorian Partnership for Advanced Computing (VPAC) for providing computational facilities.

I would like to acknowledge Mr. Adrian Varano. Thank you for accepting to read this thesis. Your comments and suggestions have greatly improved this body of work.

I would like to say thank you to all the people in Molecular Modelling and Simulations Group at RMIT for all their helpful discussions, for making me laugh and creating such a great environment to work in. We have shared some great experiences, and you are all remarkable people.

On a personal note, I would like to thank my family for their understanding and patience. Thank you to my brother for being the one person I turn to when in need.

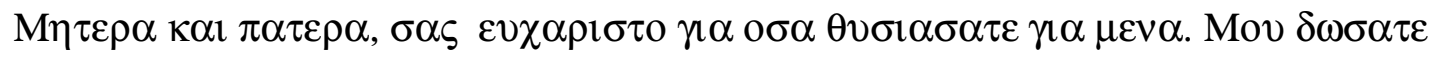




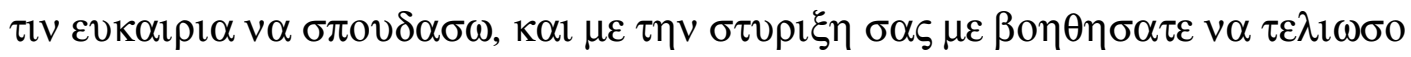

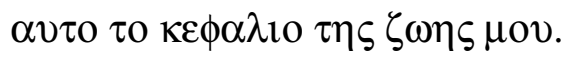

I would like to say a big thank you to my extended family (Nonna Elena and Iolanda, Nonno Frank and Vincenzo, Gessi and Marie). You have brought so much happiness in my life. Thank you for all the countless dinners, and laughs. Marie and Gessi, thank you for putting up with me for so long, and being my family away from family.

And last but definitely not least, I would like to thank my partner Olivia. It has been a long and difficult journey, and at the toughest times you have helped me rise above. You motivated me, and inspire me. Thank you. 


\begin{abstract}
This project involves atomistic level modelling of the interactions of carbonaceous contaminants with polyester based surfaces in order to study the mechanism by which airborne and rain-carried carbon particles adhere to polyester based paints, and identify key surface properties that may aid in the development of dirt repellent paint coatings. A detailed description of the motivation for this project, and a literature review on important experimental discoveries in the areas of polymer surface modification and computational modelling of polymer surface and polymer/carbon interfaces are presented in Section 1. Also, additional literature reviews are included in the introductory sections of Chapters 4 to 7 pertinent to the subject matter in question.

Classical molecular mechanics (force-field methods) is employed to describe the physical interactions between the polymer surfaces and carbon species, and molecular dynamics is used to obtain time and temperature-dependent properties of these polymer/carbon systems. A detailed description of the computational techniques is included in Section 2

A fully-atomistic polyester surface model is used to simulate the paint surface, and a range of carbon particles (graphite, amorphous carbon and fullerene $\mathrm{C}_{60}$ ) are used to simulate the airborne contaminants. The strength of adhesion between polymer and contaminant is characterised by a procedure that simulates the instantaneous separation of the polymer/carbon interface, allowing determination of the work of separation. This property is related to the ideal thermodynamic work of adhesion. In this project we explore nanoscale modifications (hydrophobic and hydrophilic treatments) of the polyester surface that potentially reduce the strength of adhesion (work of separation) between polymer and carbon adding value to polymer coatings where contamination protection is desirable. We have included a detailed description of the polymer surface models, organic contaminants, nanoscale modifications and the procedure used to evaluate interfacial adhesion in Section 3.

An important property utilized by natural stay-clean plants, such as the Lotus leaf, is nanoscale surface roughness. In Section 4 of this study, we investigate the effects of atomic-scale roughness combined with chemical surface modifications on adhesion between polymer and amorphous carbon (an industrial char derived contaminant).
\end{abstract}


Modifications to the polyester surfaces include the addition of hydroxyl $(\mathrm{OH})$, carboxyl $(\mathrm{COOH})$, and fluorine $(\mathrm{F})$ functional groups at varying levels of surface coverage. Also, in Section 4, we employ a procedure that approximates relaxation (energy-minimisation) and achieves restructuring of the modified surfaces. As we will show, surface reorganisation has an important impact on the effectiveness of our surface modifications.

Aging is an important phenomenon in polymer science, whereby treated coatings tend to recover to their natural state after some time. To investigate the effects of aging on our functionalised polyester surfaces, in Section 5, we undertake molecular dynamic (MD) simulations of carbon/polyester interfaces at $400 \mathrm{~K}$ (just above the glass transition temperature of polyester). We show that physico-chemical changes of the polymer surfaces that occur during aging, significantly impact on the strength of adhesion between polymer and carbon contaminant.

We extend our investigations of contaminant adhesion to include an aqueous environment by modelling the interaction of fullerene (an ideal model of a soot derived particulate) with polyester surfaces in water (Section 6). A series of MD simulations are undertaken with the fullerene positioned at distinct locations above the hydrated polymer surfaces. In this section, we show that a specific combination of physical and chemical properties can prevent fullerene from adhering to polyester in water.

Finally, we develop a computational methodology to chemically crosslink the surface of fully atomistic polymer models (Section 7). Surface crosslinked polyesters are constructed using hexamethylene and isophorone di-isocyanate crosslinkers. In this section, we also introduce a methodology to simulate nanoindentation of the crosslinked films, whereby a fullerene is used to probe the hardness of different regions of the polymer surface. Through this in-silico nanoindentation experiment, we not only determine the surface hardness of the polymer film but also calculate the work of adhesion between polymer and fullerene. This enables us to explore the effects of surface hardness on adhesion between polymer and carbon.

From the knowledge gained, we have developed design principles which we believe will lead to the development of novel dirt-repelling paint surfaces. These principles are summarized in the Section 8 of this study. Our future work is presented in the final section of this thesis. 


\section{Chapter 1}

\section{Introduction / Literature Review}

\subsection{Overview}

In this chapter, we detail the motivation for this research, highlighting the need for the development of self-cleaning polymer coatings. We present the two main categories of self-cleaning surfaces that are encountered in nature and which have provided the inspiration for many novel stay-clean materials; super-hydrophobic and superhydrophilic surfaces. In Section 1.4, through a detailed review of experimental literature, we present the critical properties which influence the hydrophobic and hydrophilic nature of the polymer surface. We detail some of the novel approaches and difficulties encountered in tailoring polymer surfaces to achieve specific properties. In Section 1.5, we review the previous research in the atomistic modelling of polymer surfaces and interfaces. Lastly, the project aims are presented in Section 1.6. 


\subsection{Background and Motivation}

Building facades are often exposed to extreme and fluctuating climates for long periods and this can often result in staining and discolouring of the paint surface. ${ }^{1,2}$ One major cause of discolouration is in the form of airborne and rain-carried carbonaceous particles that adhere to the paint coating. ${ }^{2}$ Carbonaceous particles arise in the atmosphere from biomass and biofuel combustion, ${ }^{3}$ and these particles can exhibit varying sizes that range from microns to nanometres. Polymers are generally most tacky at temperatures around their glass transition temperature, and under these conditions the particles can embed themselves into the polymer paint coating. This type of adhesion is persistent and the surface stains cannot be easily removed. This discolouration is a major problem for manufactures of painted building products and a solution to the problem, would be of very high commodity. In addition to targeting the issue of dirt-contamination, a solution to this problem would aid significantly the development of self-cleaning polymer coatings. These are highly sought after materials that can be used in an extensive range of applications, such as semiconductor, pharmaceutical and ophthalmic products. ${ }^{4}$ The emergence of nanotechnology has provided a potential avenue for the fabrication of selfcleaning coatings which has further invited interest in their design and application.

This research was supported by the Australian Research Council Linkage scheme with Bluescope Steel, as the industry partner. Bluescope Steel are one of the world's largest manufactures of pre-painted steel materials. The company is most recognisable for their Colorbond ${ }^{\circledR}$ products, which has revolutionised Australian building design. Colorbond ${ }^{\circledR}$ is a multi-coated system with a cured polyester based top-coat which has been specifically designed to deliver outstanding anti-corrosion performance, maximum resistance to chipping, peeling and cracking, and high solar reflectance which helps reduce the building's energy load. It is of general belief that the performance of this coating would be further enhanced with the development of a mechanism that targets dirt resistance. It is imperative that the development of such a mechanism ensures that the current properties of the coating are maintained. Therefore, an innovative approach must be employed that modifies the existing paint coating, imparting dirt-shedding properties to the surface, while at the same time, maintaining current properties of the bulk. In this 
project, we employ a combination of molecular mechanics and dynamics to theoretically investigate surface modification of polyester coatings for improved contamination resistance. We develop key concepts that will hopefully aid the synthesis of a stay-clean polymer surface.

a) Super-hydrophobic self-cleaning surface b) Super-hydrophilic self-cleaning surface

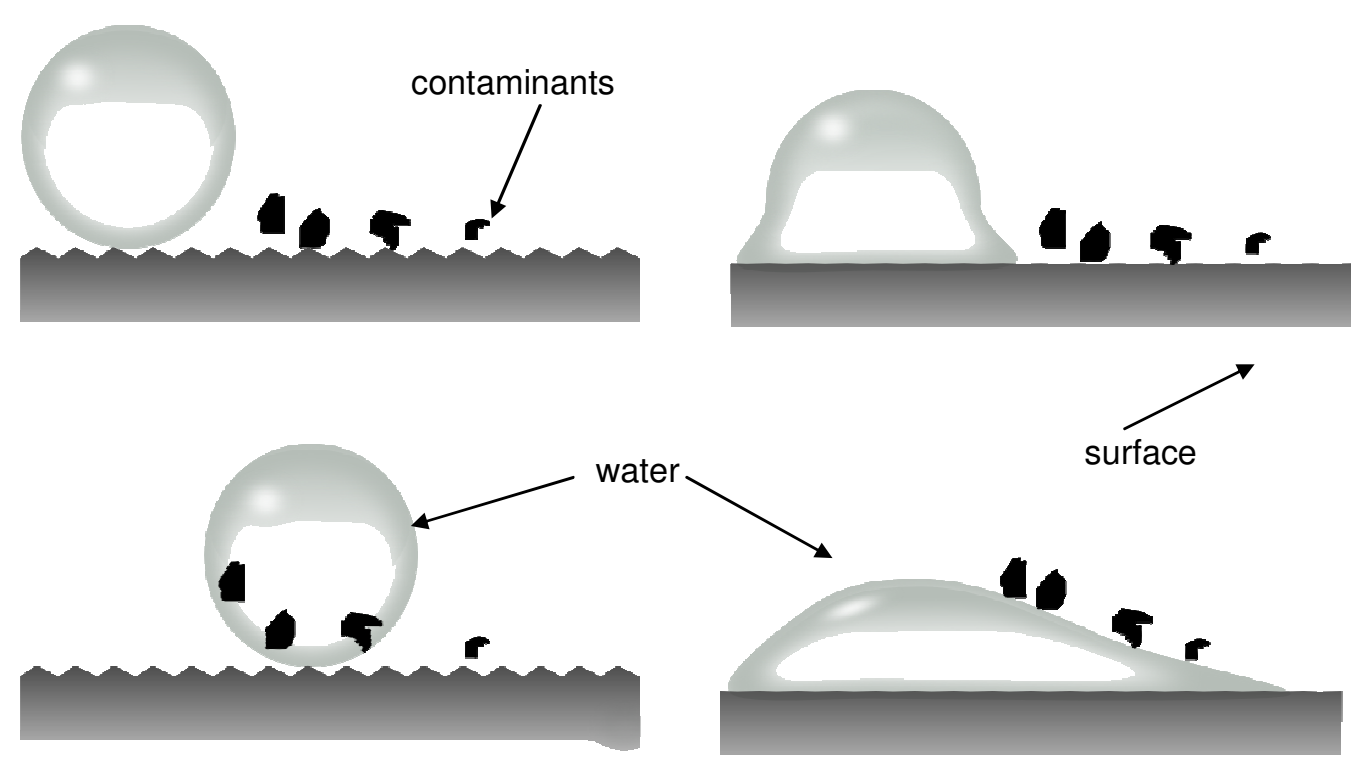

Figure 1.1 Schematic illustration of self-cleaning surfaces. a) The Lotus effect combines natural hydrophobicity with a complex surface structure to ensure water droplets easily roll-off the surface carrying with them unwanted particulates. b) On a super-hydrophilic self-cleaning surface, water droplets spread forming a thin film of water, lifting contaminants from the surface.

\subsection{Natural Self-Cleaning Surfaces}

In recent years, there has been a considerable amount of work on the development of contamination resistant coatings. For example, Lotusan ${ }^{\circledR}$ is a commercially available silicon based paint, that employs the concept of super-hydrophobicity (very low affiliation with water) to remain clean. ${ }^{4,5}$ The criteria that characterise superhydrophobic surfaces are a very high water contact angle, defined as the angle between a water drop and the surface and a low roll off angle, defined as the inclination angle at which a water drop rolls off the surface. ${ }^{6}$ The most well known example of natural super-hydrophobicity occurs on the leaves of the Lotus flower (Nelumbo nucifera). ${ }^{7}$ It is 
this flower that has provided the inspiration for the development of many self-cleaning surfaces such as Lotusun ${ }^{\circledR}$. The Lotus plant is a species of flower that typically grows in swamps and shallow waters in eastern Asia and eastern North America. Despite its 'dirty' habitat, the leaves of the Lotus flower remain clean. From the leaves protrude papillose epidermal cells of micro-scale length, which in turn are covered by nanoscale epicuticular wax structures. The wax structures are naturally hydrophobic, and in combination with the multiple-length scale surface roughness ${ }^{8}$ ensure water droplets do not penetrate in between the grooves of the leaf. In fact, the contact angle observed at the leaf of the lotus plant exceeds $150^{\circ} .^{9}$ A slight inclination in the leaf causes the water drops to roll of the surface, carrying with them any particles of dirt. This stay-clean mechanism is commonly referred to as the Lotus Effect and is schematically represented in Figure 1.1a (previous page).

Another category of self-cleaning coatings is super-hydrophilic surfaces ${ }^{4}$ which in contrast to their hydrophobic counterparts, exhibit a strong affiliation with water and are therefore characterised by very low water contact angles (less than $30^{\circ}$ ). While superhydrophobic coatings are inspired by the self-cleaning properties of plant leaves, hydrophilic coatings have parallels with photosynthesis, using sunlight to drive chemistry. The world's first self-cleaning window, Pilkington Active ${ }^{\mathrm{TM}},{ }^{10}$ has a smooth hydrophilic coating based on Titania $\left(\mathrm{TiO}_{2}\right)$, as does PPG's Sunclean ${ }^{\mathrm{TM}}$. Titania acts to clean the window in sunlight through two distinct mechanisms. The first, is that exposure to ultraviolet (UV) light causes the breakdown of dirt (photocatalysis). The second mechanism is that UV radiation cause oxidation which in turn induces sites on the surface that favour water adsorption. Water droplets thus spread, forming a thin film of water on top of the coating, lifting contaminants from the surface. ${ }^{11}$ The hydrophilic self-cleaning effect is schematically shown in Figure 1.1b. This hydrophilic surface approach has been applied to fabricate a soil resistant exterior paint coating suitable for use on military aircraft. ${ }^{2}$ This particular coating is a transparent silica (silicon dioxide) film that is applied to an existing paint surface, rendering it hydrophilic. It is proposed that good soil resistance is achieved by the fact that atmospheric particles adhere less strongly to the hydrophilic surface, enabling much easier clean up. Whilst this silica film leads to promising results, such stay-clean systems suffer from the obvious disadvantage 
of requiring an additional coating to be applied which can be time consuming and very costly. The most ideal solution and cost-effective approach, is to incorporate a dirtshielding mechanism built into the existing polymer paint coating.

\subsection{Parameters Affecting Adhesion and Wettability}

Understanding surface properties such as wettability and adhesion is critical for controlling the stay-clean mechanism of polymer surfaces. Adhesion and surface wettability are related by the surface free energy $(\gamma)$ which is the excess energy at the material's surface compared to its bulk, measured in vacuum. Since all media attract each other, formation of a surface is energetically unfavourable and so the surface free energy is always positive. It is equal to the energy required to increase the surface by a unit area, and has conventional units of $\mathrm{mJ} \mathrm{m}^{-2}$.

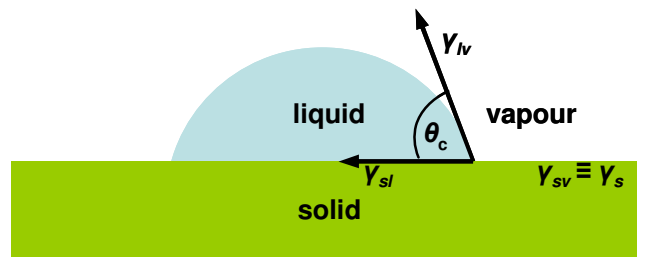

Figure 1.2 Illustration of a liquid drop on a solid surface forming a contact angle $\theta$. According to the Young-Dupré equation (eq. (1.1)), the contact angle is related to the surface and interfacial free energies $(r)$ of the comprising media.

The link between surface free energy and wettability first arose from the work of Thomas Young ${ }^{12}$ in the nineteenth century. He devised the theory that the contact angle $\left(\theta_{c}\right)$ of a drop of liquid on a solid surface (Figure 1.2) is dictated by the surface energies through the following expression known as Young's equation:

$$
\gamma_{s l}+\gamma_{l v} \cos \theta_{c}=\gamma_{s v}
$$

where $\gamma_{s v}$ and $\gamma_{l v}$ are the surface energies of the solid and liquid in vapour respectively, and $\gamma_{s l}$ is the interfacial energy between liquid and solid. Young's equation provides a means for experimentally determining the surface energy of solid materials through contact angle measurements. Highly hydrophobic materials, which form water contact angles well above $90^{\circ}$ are usually characterised by relatively low surface free energies. On the other hand, hydrophilic materials which form water contact angle below $60^{\circ}$ 
exhibit relatively high surface free energies. Presented in Table 1.1, are the surface free energy values of several polymers.

Table 1.1 Surface Free Energy of Common Polymers Measured at $20^{\circ} \mathrm{C}$.

\begin{tabular}{|c|c|}
\hline Polymer & Surface Free Energy, $\gamma\left(\mathrm{mJ} \mathrm{m}^{-2}\right)$ \\
\hline Poly(tetrafluoroethylene) (PTFE) $)^{13-18}$ & $19-20$ \\
\hline Polyethylene $(\mathrm{PE})^{17,19,20}$ & $33-37$ \\
\hline Polycarbonate $(\mathrm{PC})^{21,22}$ & $40-43$ \\
\hline Poly(ethylene terephthalate) $(\mathrm{PET})^{20,23-26}$ & $43-45$ \\
\hline Polyglycine (Nylon 2$)^{20,27}$ & $50-51$ \\
\hline Poly(2-hydroxyethyl methacrylate) $)^{28,29}$ & $55-58$ \\
\hline
\end{tabular}

The majority of polymers are hydrophobic with surface energy values in the range of approximately 20 to $50 \mathrm{~mJ} \mathrm{~m} \mathrm{~m}^{-2}$. Moderately hydrophobic polymers such as polycarbonate and poly(ethylene terephthalate) exhibit water contact angle close to $80^{\circ}{ }^{18,30}$ while poly(tetrafluoroethylene), regarded as the benchmark of highly hydrophobic polymers, forms contact angles as high as $105^{\circ} .{ }^{17,31}$ On the other end of the scale, there exist several polymers which are relatively more soluble in water such as polyglycine and poly(2-hydroxyethyl methacrylate), exhibiting higher surface free energy values (see Table 1.1).

In addition to wettability, surface energy is also regarded as fundamental to understanding adhesion. In practice, ideal thermodynamic adhesion is defined by the work of adhesion $\left(W_{a d h}\right)$. This is the free energy change, or reversible work required to separate an interface into two free surfaces in vacuum, ${ }^{13}$ and is valid for both solid and liquid interfaces. The work of adhesion is mathematically expressed by the Dupré equation, ${ }^{32}$

$$
W_{a d h}=\gamma_{1}+\gamma_{2}-\gamma_{12}
$$

where $\gamma_{1}$ and $\gamma_{2}$ are the surface free energies of materials 1 and 2, and $\gamma_{12}$ is the interfacial energy between the two materials. This fundamental relationship suggests that 
by changing the surface free energy and/or wettability, we can alter the material's adhesive properties. Jarvis and Pethica studied the influence of surface wettability on adhesion. ${ }^{33}$ For their work, adhesion was measured between a hydrophobic diamond tip and silicon surfaces of varying wettability, at a humidity of $40 \%$, using atomic-forcemicroscopy (AFM). Their results showed that a hydrophobic treated silicon surface exhibits a strong adhesion with the hydrophobic tip, highlighting the strong affiliation between hydrophobic elements. However, in the case of a hydrophilic silicon oxide surface, the formation of a thin water layer on the silicon film results in a relatively weak adhesion between tip and sample. Bingeli and Mate $^{34}$ also showed via AFM measurements, that adhesion between surfaces can be controlled by altering the surface wettability of the materials. Frisbie et al. ${ }^{35}$ fabricated surfaces comprising of selfassembled-monolayers (SAMs) of hydrophobic $\mathrm{CH}_{3^{-}}$or alternatively hydrophilic $\mathrm{COOH}$ - terminated end groups. They investigated the strength of adhesion of each modified surface with a similarly functionalised AFM tip. They found that the adhesive interaction between the functionalised tip and sample exhibited the following trend: $\mathrm{COOH} / \mathrm{COOH}>\mathrm{CH}_{3} / \mathrm{CH}_{3}>\mathrm{COOH} / \mathrm{CH}_{3}$. Thus, the interaction between hydrophilic groups $(\mathrm{COOH})$, which were capable of forming hydrogen bonds, was stronger than between hydrophobic groups $\left(\mathrm{CH}_{3}\right)$ or mixed groups.

The most commonly used method to adjust the surface energy of a material and render it hydrophobic or hydrophilic is through chemical surface modification i.e., chemically changing the surface constitution of the material. Fluorine is considered the material of choice for improving the hydrophobicity of polymer surfaces. ${ }^{36,37}$ The small size, high electronegativity, low polarizability of fluorine atoms and strong fluorinefluorine repulsion results in weak intermolecular attraction between fluorinated polymer chains and thus, relatively low surface energies. The most well known example of fluorinated polymers is poly(tetrafluoroethylene) (PTFE), which due to its strong hydrophobicity, is used for non-stick coatings in cookware (Teflon®).

Plasma source modification is the most commonly used method to implant fluorinecontaining groups to the surface of polymers, improving their hydrophobicity. ${ }^{38-42}$ In a recent article, ${ }^{43}$ plasma source ion implantation was used to modify the surface of ethylene-vinyl alcohol $(\mathrm{EVOH})$ copolymers. In high humidity environments, these 
copolymers naturally adsorb a large amount of water, and so fluorine modification was undertaken with the hope of reducing water retention. Results from this study showed that $\mathrm{CF}_{4^{-}}$plasma treatment did in fact improve the hydrophobicity of the polymer surface, due to the formation of fluorine-containing functional groups $\mathrm{CF}, \mathrm{CF}_{2}$ and $\mathrm{CF}_{3}$.

However, incorporation of fluorine groups to polymer surfaces does not always result in an increase in hydrophobicity, with several studies ${ }^{44-48}$ showing that direct fluorination can often increase the surface wettability of polymers. In direct fluorination the surface is exposed to an $F_{2}$ gas mixture. The level of fluorine content attained with this method is much lower than the traditional plasma treatment, and provided that the untreated polymer is sufficiently non-polar to begin with, direct fluorination can often result in an increase surface polarity, and therefore an increase in the surface energy and wettability of the polymer film. Also, depending on the $F_{2}$ gas mixture and the secondary reactions than can occur under humid conditions, direct fluorination can also lead to the formation of highly polar groups such as $\mathrm{COOH}$, further increasing the wettability of the film. In one study ${ }^{48}$ poly(phenylene oxide) films were fluorinated with varying fluorine concentration feed. For low level modifications (low fluorine concentration), the surface energy of the polymer was shown to increase compared to the untreated film due to the slight increase in polarity of the film. XPS results showed that during modification, hydrogen surface atoms were substituted with fluorine atoms. Fluorine has a higher electronegativity that the original hydrogen that is replaced. Thus, one would expect the fluorination would indeed result in an improvement in the polarity of the film. However, as the fluorine concentration was further increased, the surface energy of the film began to decrease highlighting that wettability can be controlled by adjusting the concentration of fluorine-containing groups.

Among the reviewed literature, a commonly used approach to increase the surface free energy of polymer films and improve the hydrophilic character is by increasing the surface content of highly polar groups such as $\mathrm{OH}, \mathrm{COOH}$, or nitrogen containing functional groups like $\mathrm{C}-\mathrm{N}$ or $\mathrm{C}=\mathrm{N}$. These groups can hydrogen bond with surrounding water molecules, and increase water uptake. In the work of Vandencansteele et al. ${ }^{49,50}$ polytetrafluoroethylene (PTFE) and polyvinyl fluorine (PVF) surfaces were exposed to nitrogen plasma leading to an increase in the hydrophilicity of the surfaces. These 
changes were directly related to the amount of nitrogen polar groups, grafted to the surface. Unexpectedly, when the PTFE surface was exposed to $\mathrm{O}_{2}$ plasma, ${ }^{49}$ a reduction in the surface hydrophilicity was noted, attributed to the fact that no oxygen surface grafting actually took place, and that oxygen plasma induced only etching of the surface. As will be shown later, etching and changes in surface roughness, can have a significant impact of the free energy of the surface. In another example, ${ }^{43}$ oxygen $\left(\mathrm{O}_{2}\right)$ plasma was used to modify the surface of ethylene-vinyl alcohol (EVOH). In this case, the oxygen plasma treatment did improve the hydrophilic character of the surface, as indicated by contact angle measurements, due to the conversion of $\mathrm{C}-\mathrm{H}$ bonds in the polymer to more polar groups, such as $\mathrm{C}-\mathrm{O}, \mathrm{C}=\mathrm{O}$ and $\mathrm{C}=\mathrm{C}-\mathrm{O}$. Furthermore, the hydrophilically treated EVOH polymer showed an increased strength of adhesion with acrylic tape owing to the incorporation of the surface oxygen species. In a recent article, ${ }^{51}$ a polymer film of varying hydrophilicity was fabricated by partially masking the film during Ar plasma treatment. This introduced a wettability gradient along the surface marked by a varying contact angle of $150^{\circ}$ for the untreated area down to $0^{\circ}$ for the highly-modified region. The large increase in wettability was associated, with the production of radicals on the material surface, followed by formation of unstable species. These unstable compounds produced various oxygen-based polar functionalities (hydroxyl and carboxyl groups) by reaction with additional oxygen in the air, increasing the hydrophilic character of the surface.

Another important parameter requiring careful consideration with regards to adhesion and wettability is surface roughness. Mizes et al. ${ }^{52}$ used AFM to investigate the relationship between surface roughness and adhesion, and found that the slightest variation in nanoscale surface roughness caused fluctuations in adhesion strength between surfaces. The lotus leaf, which is regarded as the 'holy grail' of self-cleaning surfaces (see Section 1.3), utilizes its nano-scale and micro-scale roughness to ensure dirt particles do not penetrate in between its surface grooves. 


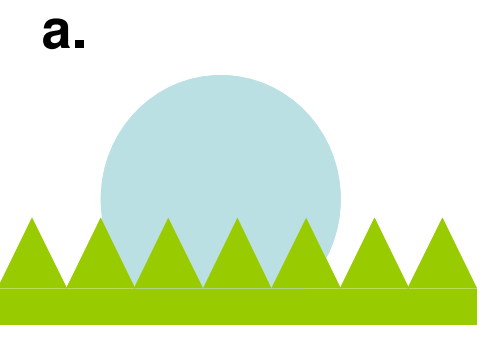

Wenzel's Model b.

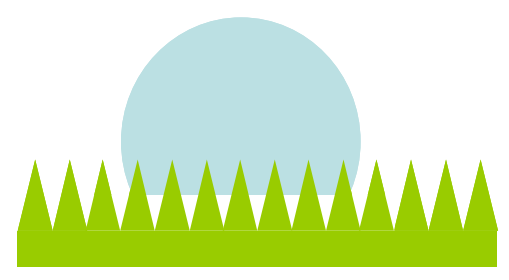

Cassie-Baxter Model

Figure 1.3 Schematic illustrations of the Wenzel and Cassie-Baxter constructions, which determine the influence of roughness on the wettability of the surface.

The fundamental relationship between surface roughness and wettability was first introduced by Wenzel, ${ }^{53}$ with the following relationship:

$$
\cos \Phi=r \cos \theta_{c}
$$

where $r$ is the roughness factor defined as the ratio of true surface area to projected surface area $(\mathrm{r}>1), \Phi$ is the new contact angle as a result of the roughened surface, and $\theta_{c}$ is Young's contact angle formed on the chemically equivalent smooth surface (i.e., intrinsic contact angle). This relationship assumes that the grooves or voids on the surface are accessible to the opposing liquid (as shown in Figure 1.3a) It can be seen from eq. (1.3) that if the intrinsic contact angle $\left(\theta_{c}\right)$ is less than $90^{\circ}$, i.e., the surface is initially hydrophilic, roughening its exterior will reduce the contact angle and promote further wetting. ${ }^{54}$ Conversely, if the surface is naturally hydrophobic, (i.e., contact angle $\theta_{c}$, is larger than $90^{\circ}$ ) then roughening the surface will in fact reduce wetting and enhance the materials hydrophobicity. Wenzel's relationship can hold true for moderately rough surfaces exhibiting protrusion of micro-scale to nano-scale length. Jung and Bhushan ${ }^{55}$ measured the static contact angle of water with roughened hydrophilic poly(methylmethacrylate) (PMMA) and hydrophobic polystyrene (PS) surfaces. The roughness factor $(r)$ was determined for each surface, and the data showed that the PMMA surface became more hydrophilic with increasing $r$, while for the PS surface, it became more hydrophobic, in accordance with Wenzel's theory (eq.(1.3)).

Wenzel's construction however, becomes increasingly unconvincing when the scale of surface roughness approaches the molecular level. ${ }^{56}$ Cassie and Baxter ${ }^{57}$ extended 
Wenzel's treatment to accommodate finer surface roughness, where the voids are not entirely accessible to the liquid and so interfacial air gaps are present (Figure 1.3b). The contact angle is now given by:

$$
\cos \phi=f_{s l} r \cos \theta_{c}+f_{s l}-1
$$

where $f_{s l}$ is the fraction of the solid surface area that is wetted by the liquid. We note that usually $f_{s l}<1$ and in Wenzel's case, $f_{s l}=1$. Close inspection of eq. (1.4) shows that in the Cassie-Baxter regime, there is an improvement in hydrophobicity if the surface is roughened, irrespective of the contact angle of the original smooth surface $\left(\theta_{c}\right)$. Hence, hydrophobic surfaces can be made superhydrophobic by introducing fine-scale roughness that reduces the contact area between liquid and solid (as in the case of the Lotus leaf). Alternatively, hydrophilic films, can be made super-hydrophilic by nanoscale smoothing of the surface. ${ }^{58}$

A lot of effort has gone to improving liquid repellence by combining natural hydrophobicity with geometric factors, which the lotus leaf employs so well to remain clean. Coulson et al. ${ }^{56}$ described an approach whereby oxygen plasma was used to roughen the surface of naturally hydrophobic PTFE substrates giving rise to ultra-waterrepellence with contact angles exceeding $150^{\circ}$. Similarly, Shiu et al. ${ }^{59}$ treated a Teflon ${ }^{\circledR}$ film with oxygen plasma and obtained a superhydrophobic surface with a contact angle of $168^{\circ}$. Inoue et al. ${ }^{60}$ employed Ar-ion bombardment to fabricate a super-hydrophobic PTFE surface causing a contact angle change from $103^{\circ}$ to $170^{\circ}$.

These examples demonstrate the applicability of Cassie's theorem. However, a drawback of the Cassie-Baxter or Wenzel constructions is that they assume a homogenous roughness, when in fact surfaces are naturally heterogeneous i.e., exhibit multiple length-scale roughness. It has been revealed ${ }^{7,8,61}$ that the surface heterogeneity of the Lotus leaf, in the form of double-length pillars, not only enhances the leaf's hydrophobicity, but allows dirt carrying droplets to easily roll-off its surface by reducing the contact angle hysteresis as well as the adhesion between water and surface. Roughness of varying length-scales is of paramount importance in applications where self-cleaning is desired. In recent years, considerable effort has been undertaken in synthesizing polymer surfaces that replicate the multiple-length topography of the lotus 
leaf, and increase the mobility of water droplets on the exterior of the materials. Zhang et al. ${ }^{62}$ fabricated multiple length roughness on a perfluoropolyether surface, using a pretextured aluminium surface as a mould (template). The polymer surface, which is naturally hydrophobic, exhibited a change to superhydrophobic after texturing, evident by the high contact angles $\left(170^{\circ}\right)$, and low contact angle hysteresis. Ming et al. ${ }^{63}$ prepared a dual-length roughened polymer surface, by covalently grafting onto the polymer, silica raspberry-like nano-particles, functionalised with amine groups. The roughened surface was subsequently chemically modified with a layer of poly(dimethylsiloxane) (PDMS). The chemical modification combined with the multilength surface topography resulted in a superhydrophobic film of high contact angle and low roll off angle.

One of the biggest obstacles facing synthetic construction of stay-clean surfaces is that of stability. The mobility of polymer chains enables polymer surfaces to respond to interfacial forces adapting their surface chemical structure to their environment. By rotational and translational motions of the chain segments, the interfacial free energy $\left(\gamma_{12}\right.$ from eq. (1.2)) between the polymer and its environment can be minimised. ${ }^{64,65}$ The attractive or repulsive interaction force between polymer and its contacting phase determines the entity of polymer chain rearrangement, which is driven by the difference in the surface energy of the polymer surface and the contacting medium. Specifically, in a non-polar environment such as air, polymers minimise the density of polar groups at the surface, ${ }^{66}$ whereas in an aqueous environment it is energetically more favourable for the polymer surface to become enriched in polar groups and reduce the density of nonpolar groups. ${ }^{67}$ Modification of a polymer surface typically creates a surface that is not at equilibrium. Freshly modified polymer surfaces are therefore unstable and lose, within hours or days, partially or completely, the properties conferred by surface treatments.

For instance, Zhang et al. ${ }^{68}$ investigated the anti-fouling behaviour of smooth and nanolength roughened hydrophobic silica surfaces. While initially, the roughened samples exhibited superior anti-fouling behaviour to their smooth equivalents, after a period of time immersed in water ( $>1$ month), the anti-fouling properties of all samples significantly deteriorated due to a loss in hydrophobicity. In this article no specific detail was given regarding the reason for hydrophobic deterioration, however one can assume 
that the chemistry or structure of the surface changes due to either reorganisation of the existing surface or attachment of new molecules from solution. In another study, ${ }^{69}$ superhydrophobic polymer surfaces with contact angles as large as $165^{\circ}$ were engineered by combining hydrophobic surface chemistry and nano-roughness. A double length roughness was achieved by incorporation of micro and nanoscale particles which help reduce the contact angle hysteresis, and as a result water drops roll off the surface at very low slide off angles (below $1^{\circ}$ ). However these 'stay-clean coatings' were shown to exhibit poor stability, due to poor adhesion with the substrate, and could easily be removed by hand. Attempts were made to improve the stability of the coatings using various binders, however, these were met with limited success. Even the durability of self-cleaning silicon based surfaces, like Lotusan ${ }^{\circledR}$, are affected after long term exposure in natural environments. For instance, Zimmerman et al. ${ }^{70}$ investigated the durability of superhydrophobic a silicone coating by exposing these surfaces for at least 12 months of outdoor weathering. Results showed that the roll of angle is particularly sensitive to outdoor weathering with the weathered samples displaying an increased contact angle hysteresis. This change has been shown to deteriorate the long-term self-cleaning ability of the coating.

Attempts have been undertaken to fabricate superhydrophobic surfaces which are durable in air and water. Zimmeraman et al. ${ }^{70}$ showed that surface stability of silicone coatings can be improved though annealing of the films. Lee et al. $^{71}$ engineered nanoscale rough PTFE surfaces which exhibited high contact angles, and low roll off angles using a pre-textured aluminium surface as a template similar to Zhang et al. ${ }^{62}$ In this case, the super-hydrophobic PTFE surfaces were unaffected by aging in air and water as a result of curing during nanoroughening. The curing process inevitably resulted in crosslinking of the polymer film leading to increased rigidity and stability of the surface. In another study, ${ }^{72}$ stable super-hydrophilic surfaces were engineered, consisting of composite thin films of $\mathrm{TiO}_{2}$ and $\mathrm{SiO}_{2}$. By embedding $\mathrm{TiO}_{2}$ nanocrystallites into the material, its hydrophilicity persisted for up to eight weeks in ambient conditions, but eventually the hydrophilic character of the surface did deteriorate as a result of adhesion of carbon contaminants onto the surface of the film. 
Despite the relatively extensive experimental observations, it is difficult to elucidate the precise properties required to fabricate stay-clean polymer coatings. To determine such properties one requires a thorough understanding of the nature of interactions that occur between the contaminants and polymer coating. In this project, we use atomistic simulation to gain insight into the detailed interactions of polymer surfaces with carbon contaminants.

\subsection{Modelling Studies of Polymer Surfaces and Interfaces}

The technique most often used to model atomistic structure and properties of polymeric materials is the empirical force-field method. This is because ab-initio methods are too computationally expensive to model large systems that are typically associated with polymers. The force-field approach has extensively been tested for organic materials, including synthetic polymers, predicting properties such as cohesive energies, ${ }^{73}$ surface $^{74}$ and interfacial energies, ${ }^{75}$ elasticity $^{76}$ and diffusion in quantitive agreement with experiment. In the force-field approach, the energy of the system is modelled by a potential that describes the bonded interactions between atoms, associated with variations in bond lengths and angles, and non-bonded interactions such as van der Waals and electrostatic. The specific mathematical forms of the potential terms can vary in different force-fields and are specific for each type of interaction. The detailed description of one of the more advanced and comprehensive force-fields to date

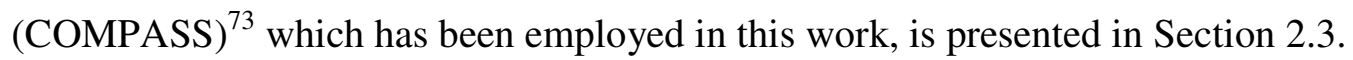

Generally speaking, force-fields can be categorised according to the specific phase or class of materials they has been developed for. Generic force-fields such as $\mathrm{UFF}^{77}$ and Dreiding $^{78}$ are designed to cover molecules of a large combination of elements in the periodic table. In the UFF force-field, simple functional forms are used for the diagonal terms of the force-constant matrix while in the Dreiding force-field simple harmonic potentials are used to describe valence interactions. Because of the generality of parameterization, these force-fields are normally expected to yield reasonable predictions of molecular structure but erroneous predictions of vibrational frequencies, ${ }^{73}$ which somewhat restricts their potential application. 
Some of the more developed force-fields include those originally designed for biological applications. The most commonly used examples include AMBER, ${ }^{79}$ CHARMM $^{80}$ and OPLS. ${ }^{81}$ In newer versions of these force-fields, there has been a shift in focus to predict condensed-phase properties of polymers. Jorgensen and co-workers published OPLS/AMBER force field ${ }^{81}$ in which the authors extended their well-known OPLS force-field approach from a united atom model to an explicit all-atom force field. However similar to the category of a generic force-field, these potentials maintain simple functional forms and lack cross-coupling terms and high-order (cubic and quartic) force constants which are often required for a more accurate prediction of vibrational frequencies. For this reason class II force-fields were developed such as MM3, ${ }^{82-84}$ MM4, ${ }^{85-87}$ CFF93, ${ }^{88-90}$ PCFF and COMPASS ${ }^{73}$ that comprise of cross-terms. The parameters for these class II force-fields were derived using quantum ab-initio data or high-quality experimental data. With the great flexibility of the functional forms and immense quantity of data used to accurately parameterize these force-fields, the calculation errors are within the experimental precision. For a particular application, a force-field has to be chosen based on comprehensive tests of its ability to predict known properties of the system in question. An example of such testing can be found in the work Todorova et al. ${ }^{91}$

An early example of the use of empirical force-field methods to study polymeric properties, include an investigation on the restructuring of a poly(ethylene)terephthale in solution. ${ }^{92}$ The investigation was undertaken to help elucidate some experimentally observed trends such as polymer swelling and contact angle hysteresis. The rotation isomeric state model (RIS) ${ }^{93}$ was employed to obtain minimum energy conformations of the polymer in solution as a function of torsion angles, according to a Boltzmann distribution. The results obtained from this early model, are in agreement with experiment and indicate that the polymer preferentially adopts an extended conformation in solution, possibly due to hydrogen bonding between ester groups of the polymer and water.

One of the most influential studies involving the atomistic modelling of polymeric materials to date, is the work of Yarovsky and Evans ${ }^{94}$. In this particular study, a methodology based on force-field techniques was presented and used to construct 
realistic models of cured polymer coatings. The methodology involved firstly combining the resin component of the paint with the crosslinker component in a unit cell, and then equilibrating the mixture by undertaking molecular dynamic simulations. In accordance with the proposed crosslinking reaction and by employing an inter-atomic cut-off distance, the most probable reactive sites between the components were subsequently identified. In particular, groups separated by a shorter distance than the user-defined cutoff radius, were considered most probable to react. The groups identified were chemically reacted with new bonds formed, and the system re-equilibrated. This process was repeated iteratively until the maximum crosslinking density was achieved. Experimentally observed shrinkage of the resins was reproduced in the models and the barrier properties and adhesion of the coatings were estimated. Since then, this method or derivatives of it have been utilised to model an array of fully atomistic polymer materials. $^{76,95-106}$

Force-field methods have also been used to determine the surface free energies and work of adhesion of various hydrocarbon based polymers. ${ }^{107}$ The primary difference between the polymers studied was the type of side groups on the backbone chain. The purpose of this study was to investigate the effect of these side groups on interfacial adhesion between polymer surfaces. Firstly, amorphous constructs of polymers were generated from a single parent polymer chain in a cell under periodic boundary conditions. Molecular dynamics (MD) was subsequently used to equilibrate these polymer structures. From these amorphous arrangements, thin films were generated by extending the unit cell length in the $\mathrm{z}$ direction, followed by re-equilibration. The surface tension was then determined by the energy difference between the bulk and its equivalent film structure. Polymer-polymer interfaces were formed by bringing together the thin films, from which the work of adhesion was evaluated. While values of surface tension and work of adhesion were not in quantitative agreement with known experimental values, this article demonstrates the usefulness of molecular mechanics/dynamics to isolate contributing factors to polymer adhesion, such as sidegroup chemistry.

A number of computer simulation techniques based on molecular mechanics have been developed, capable of probing the interface between a polymer and a carbon 
surface in atomistic detail. The polymer/carbon interface is of specific interest to this study because it represents a first approximation of a typical coating/contaminant interface. For example, in the study of Mansfield and Theodorou, ${ }^{108,109}$ the interface between polypropylene and a graphite basal plane (001) was investigated. In this method multiple equilibrium states of the system were generated, each state consisting of a film of polypropylene sandwiched between two graphite planes with periodic boundary conditions in the $\mathrm{x}$ and $\mathrm{y}$ direction. The states were acquired by firstly obtaining an initial guess state structure using Monte Carlo methods. The energy minimisation algorithm was then applied to each structure, to attain minimum energy configurations (see Section 2.4 for further detail on energy minimisation). Subsequently, the models were used to estimate the work of adhesion between graphite and polypropylene, as defined by eq. (1.2). However, the work of adhesion was not measured directly, but determined using a known value of the surface energy of the polymer. Specifically, the graphite plane was displaced by a small amount towards the polymer film, and the change in potential energy per unit area was measured. This value was then combined with a known value of the surface tension of polypropylene to determine the work of adhesion which was found to be in good quantitative agreement with experiment. In a similar manner, Attwood et al., ${ }^{110}$ calculated the adsorption energy of epoxy and amine molecules with an oxidised graphite plane. Epoxy and amine are major components of paint resins. Adsorption energies of these two molecules were evaluated using forcefield techniques. Each molecule was placed close to the carbon fibre surface, and the structure energy minimised with the carbon model kept fixed. The adsorption energy was then calculated by subtracting the energy of the isolated molecule from the energy of the molecule in its absorbed state (graphite and adsorbate composite). Values of adsorption energies revealed that the epoxy molecule was more strongly attracted to the graphite plane than the amine hardener, in agreement with micromechanical testing. ${ }^{11}$ However, the calculated adsorption energy values do not directly match those attained by experiment. In this example, the chosen force-field was Dreiding, ${ }^{78}$ which has been parameterized to cover a wide range of organic and main group in-organic molecules; however, it is considered only moderately accurate in predicting geometries and conformational energies. An alternative force-field, is the $\mathrm{MM}+{ }^{112}$ which has been used 
in two studies, to determine the interfacial characteristics between carbon nanotubes and polymers in relation to fiber reinforced materials. ${ }^{113,114}$ In these studies, various composite systems were constructed comprising of polystyrene $\left(-\mathrm{CH}_{2} \mathrm{CHC}_{6} \mathrm{H}_{6}\right)_{\mathrm{n}}$ in close contact with graphene and carbon nanotube materials. The free energy of each system was evaluated as the components of the system were pulled apart (e.g., as the carbon nanotube was pulled from a polystyrene matrix). The energy difference between the initial composite system and the isolated components (the completely pulled-out configuration) was taken as the adhesion strength. In both studies, the adhesion strength was shown to arise from van der Waals and electrostatic interactions. In the work of Liao and $\mathrm{Li},{ }^{113}$ it was revealed that the adhesion strength between polystyrene and a carbon nanotube is function of material deformation and coefficient of thermal expansion. In the study of Wong et al., ${ }^{114}$ it was shown that the non-uniformity of the carbon nanotube surface results in mechanical interlocking with the polymer, resulting in a strong interfacial adhesion. The MM+ force-field was also used in a molecular dynamics study to investigate the interaction of conjugated polymers (polymers containing aromatic carbons) with carbon nanotube structures. ${ }^{115}$ The purpose of this work was to determine specific monomer structures that influence the wrapping of the polymer around the nanotube models. For polymers which have aromatic rings in the backbone chain, these aromatic rings were shown to gradually align parallel to the carbon surface in agreement with XPS studies. Compared to the Dreiding force-field, the $\mathrm{MM}+$ force-field more accurately predicts the conformational energies for the vast majority of organic compounds. However, for conjugated and particularly for heteroaromatic systems such as polyester, $\mathrm{MM}+$ is found to struggle in predicting the correct bond lengths and atomic coordinates. ${ }^{112}$

In comparison, COMPASS ${ }^{73}$ (Condensed-phase Optimized Molecular Potentials for Atomistic Simulation Studies) force-field has been more extensively parameterized, covering a wider range of organic as well as inorganic compounds, and predicting conformational energies and structural parameters to a higher accuracy. The COMPASS force-field is actually an extension of the PCFF force-field which was successfully employed by Yarovksy et al. ${ }^{94}$ to construct realistic models of paint coatings. The COMPASS force-field is ideally suited for modelling synthetic organic polymer 
coatings. More recently, the COMPASS force-field was used to investigate the interactions of carbon with polyester and silica surfaces. ${ }^{95,98,116}$ In one example, ${ }^{95}$ the carbon model used was in the form of an ideal graphite basal plane. Values of adhesion strength were attained by monitoring the change in free energy as two surfaces (e.g. graphite and polyester) were brought in close contact to form an interface. Findings of this study reveal that polyester exhibits a significantly stronger adhesion with graphite compared to silica in qualitative agreement with experiment. ${ }^{81,84}$ Also the adhesion strength and equilibrium distance between graphene planes were evaluated, and found to be in excellent quantitative agreement with experiment.

\subsection{Project Aims}

The aim of this project is to apply atomistic modelling techniques to examine the mechanism by which airborne and rain-carried carbon particles adhere to polyester surfaces, and to develop innovative ways to reduce the surface contamination. The specific aims of the project are categorised below:

- Investigate the nature of the interaction of carbon contaminants with polyester coatings.

- Understand the mode of action of currently available dirt-repelling treatments.

- Determine the role of hydrophobic and hydrophilic surface treatments for contamination protection of polymer coatings.

- Investigate the role of atomic-scale surface roughness in the protection against contamination.

- Examine the influence of aging and hydrophobic recovery on the contamination relief mechanism of treated polymer coatings.

- Examine the role of surface stability and surface crosslinking with regards to self-cleaning.

- Identify design parameters for contamination protection of polymer coatings that are applicable to painted products such as Colorbond®. 


\section{Chapter 2}

\section{Principles of Atomistic Simulations of Large Systems}

\subsection{Overview}

In this work we use atomistic simulations to provide insight into the interaction of carbon contaminant particles with polyester based surfaces at the atomic-level, and derive design principles that can be used synthesize stay-clean polymer coatings. The computational technique used to model our systems of interest is the force-field method. In this section we will detail some of the principles behind the force-field method, such as the potential energy expression and the concept of atom types. We will describe the validation process of the selected force-field, demonstrating its applicability to accurately model organic surfaces and interfaces including those related to this work. We will detail the energy minimisation process used to obtain states of minimum energy. We will also show how molecular dynamic simulations may be used to monitor the time evolution of molecular systems, generating trajectories from which time-dependent properties may be extracted. Finally, we will describe the procedures that have been employed to maintain the temperature and pressure in our simulations. 


\subsection{Introduction}

Atomistic simulation is a general term used to describe the technique involved in modelling the behavior of atoms in a material and is invariably associated with computational chemistry. Atomistic modelling combines computational simulations with fundamental laws of physics and chemistry, to provide important information about the system or material regarding its structure, conformation, energy and reactivity at the atomic level. Atomistic simulation is often used in tandem with experimentally derived data to provide insight into material structure and properties at the atomic-level. While results obtained from atomistic simulations can accurately match those obtained from experiment, a clear advantage of atomistic simulations is its ability to investigate systematic changes including extreme conditions that would otherwise be impossible to achieve experimentally. For example in atomistic simulations, one can constrain the arrangement of atoms of a material during cleavage, and obtain a measure of surface free energy that is independent of surface reconstruction. Therefore, by maintaining other influential factors a constant, atomistic simulation enables us to isolate and investigate specific features of a material. Atomistic simulations are carried out by solving equations of quantum or classical physics.

In quantum physics, the time independent Schrödinger equation describes the quantum state of the system, and solving this equation can provide molecular, atomic or sub-atomic level information about the material. The time independent Schrödinger equation of a system of particles, takes the form:

$$
\mathbf{H} \Psi(\mathbf{r})=E \Psi(\mathbf{r})
$$

where $\mathbf{H}$ is the Hamiltonian operator acting on the wave function $\Psi$, and $\mathrm{E}$ is the total energy of the system. The wave function is dependent on the position vector $\mathbf{r}$ of each particle, and the square of the wave function gives the probability of locating the particles. Solution to eq. (2.1) gives a set of eigenvalues corresponding to energy states of each particle. Schrödinger's equation is universal in that it describes all matter from biological macromolecules such as proteins, enzymes and nucleic acids, to metals and semiconductors to synthetic materials such as plastics. While Schrödinger's equation can 
be solved for a hydrogen atom, for larger systems a number of approximations are required.

The Born-Oppenheimer approximation is the cornerstone of molecular mechanics modelling. It assumes that the mass of the nuclei are significantly larger than the electrons and as a consequence, electrons are considered to adjust very fast to a change in nuclear position. In fact, from the electron "point of view", the nuclei are stationary. The implication of the Born-Oppenheimer approximation is that it allows for the motion of the electrons to be separated from the motion of the nuclei. Therefore, eq. (2.1) is solved by "freezing" the position of the nuclei and determining the energy for a given set of nuclei coordinates. Quantum mechanical modelling techniques based on eq. (2.1), such as ab-initio methods or DFT enable electronic properties and reactivity information of the system to be determined.

In contrast to quantum mechanical methods, classical approaches do not attempt to solve Schrödinger's equation. Rather, the energy of the molecular system is evaluated by a combination of potentials that describe bonded and non-bonded interactions occurring between atoms or molecules within the system. This particular approach is referred to as molecular mechanics or the force-field method. In the force-field approach, the form of the pair potentials are based on classical laws of physics such as Hooke's law, and any electronic information is provided through parameters or constants in the potential energy expression. This approach is again based on the Born-Oppenheimer approximation. The constants in the potential energy expression are determined by fitting to experimental data, or calculated through higher level ab-initio calculations. In contrast to quantum mechanical methods, the force-field approach is computationally less expensive with the trade off that it cannot provide properties directly dependent upon the electronic characteristics of atoms, such as chemical bond formation or breaking. The force-field approach can however describe physical interaction in the system, and reproduce structural, conformational, thermophysical and vibrational properties quite well.

Before embarking on a detailed description of the modelling technique used in this study, it may be useful to take a wider look at fundamental features that describe the system in question. These features effectively determine the technique that will be used 
to simulate the material. The first feature relates to the property of interest. For example, to obtain a measure of the chemical binding energy between two materials, a quantum mechanical based technique is required. However, for physical interactions such as adhesion, the application of classical based techniques will most often suffice. The next feature is the type and number of particles in the system which may pose limitations on what we are able to model. The third feature is the starting condition, detailing the initial position of the particles and their starting velocities. The starting condition strongly affects which part of phase space (position and velocity values) will be sampled. Sampling the complete phase space (all possible values of positions and velocities) is usually not possible due to limits of computer power. The last feature that is required to model our system is the interaction between particles and how this interaction varies with time (dynamic equation). This dynamical equation depends on the mass and velocity of the particles, and can adopt different mathematical forms depending on the type of particles.

The models used in this work are fully atomistic, comprising of approximately 3000 particles. These systems are far too large to be modelled by ab-initio methods or density functional theory (DFT). Alternative approaches based on Schrodinger's equation, include semi-empirical methods, which allow for relatively large models to be simulated at the electronic level by fitting several parameters to experimental data. However physical interactions are the dominant interactions within our systems, as opposed to chemical, and these types of interactions are best studied using the force-field method. In comparison to ab-initio or semi-empirical methods, the force-field approach can calculate physical properties of fully atomistic models in a fraction of the computer time. Also, longer simulation times can be investigated using the atomistic force-field approach to obtain a more accurate measure of time-dependent properties.

\subsection{Force-field Method}

\subsubsection{Force-field Energy Expression}

The force-field potential is used to evaluate the energy of the system and is expressed as a function of the nuclear coordinates. This expression can take many forms depending on the force-field type chosen. In general, the force-field energy expression is a 
combination of inter-atomic potentials summed across all pairs of atoms, that model the interaction of the system due to processes such as stretching and compressing of bonds, the opening and closing of bond angles, rotations about single bonds, as well as Coulombic and van der Waals forces. The force-field chosen in this work, COMPASS (Condensed-phase Optimised Molecular Potentials for Atomistic Simulation Studies), ${ }^{73}$ has the following functional form:

$$
\begin{aligned}
& V=\sum_{b}\left[k_{2}\left(b-b_{o}\right)^{2}+k_{3}\left(b-b_{o}\right)^{3}+k_{4}\left(b-b_{o}\right)^{4}\right]+ \\
& \sum_{\theta}\left[k_{2}\left(\theta-\theta_{o}\right)^{2}+k_{3}\left(\theta-\theta_{o}\right)^{3}+k_{4}\left(\theta-\theta_{o}\right)^{4}\right]+ \\
& \sum_{\phi}\left[k_{1}(1-\cos \phi)+k_{2}(1-\cos 2 \phi)+k_{3}(1-\cos 3 \phi)\right]+ \\
& \sum_{\xi} k_{2} \xi^{2}+\sum_{b, b^{\prime}} k\left(b-b_{o}\right)\left(b^{\prime}-b_{o}^{\prime}\right)+\sum_{b, \theta} k\left(b-b_{o}\right)\left(\theta-\theta_{o}\right)+ \\
& \sum_{b, \phi}\left(b-b_{o}\right)\left[k_{1} \cos \phi+k_{2} \cos 2 \phi+k_{3} \cos 3 \phi\right]^{(6)} \\
& \sum_{\theta, \phi}\left(\theta-\theta_{o}\right)\left[k_{1} \cos \phi+k_{2} \cos 2 \phi+k_{3} \cos 3 \phi\right]+ \\
& \sum_{\theta, \theta} k\left(\theta^{\prime}-\theta_{o}^{\prime}\right)\left(\theta-\theta_{o}\right)+\sum_{\theta, \theta_{,}^{\prime} \phi} k\left(\theta-\theta_{o}\right)\left(\theta^{\prime}-\theta_{o}^{\prime}\right) \cos \phi+ \\
& \quad(9) \quad[(10) \\
& \sum_{i, j} \frac{q_{i} q_{j}}{4 \pi \varepsilon_{o} r_{i j}}+\sum_{i, j}\left[\varepsilon_{i j}\left[\left(\frac{r_{i j}^{o}}{r_{i j}}\right)^{9}-3\left(\frac{r_{i j}^{o}}{r_{i j}}\right)^{6}\right]\right.
\end{aligned}
$$

The variables and parameters of this function are described below: This function can be divided into two categories. The first category includes the valence terms (terms 1-10), which represent the energy contribution due to changes in the internal coordinates of the system, such as the bond length, bond angle and torsion angle. The second category includes the non-bond interaction terms (terms 11 and 12), which describe interactions between non-bonded parts of the system or bonded parts with two or more intervening atoms. Non-bonded interactions include electrostatic (term 11) and van der Waals interactions (term 12). 
The first term in the force-field equation describes the energy changes due to deviations in bond length $(b)$ from the reference value $\left(b_{0}\right)$. This type of deformation commonly referred to as bond stretching, is modelled using a polynomial extension of Hooke's Law where $k$ is the spring constant.

The second term, referred to as angle bending, adopts a similar functional form to the bond stretching term and describes the energy contribution due to deviation of valence angles $(\theta)$ from their reference values. The valence angle is the angle formed between atoms A-B-C in which $\mathrm{A}$ and $\mathrm{C}$ are both bonded to $\mathrm{B}$.

The bond stretching and angle bending deformations (first and second terms) are often described as 'hard' degrees of freedom. This is because substantial energy is required to cause changes in bond length or valence angles. A far greater contribution to the relative energy of the system is achieved through deviation of the torsion angle $(\phi)$. In a chain of atoms A-B-C-D, the torsion angle is the angle between the plane containing atoms $\mathrm{ABC}$ and the plane containing $\mathrm{BCD}$. Torsional potentials are almost always expressed as a cosine series expansion, as shown by the third term is the force-field expression. Here $k$ corresponds to the height of the torsional barrier and gives an indication of the energy required for rotatation around the corresponding bond.

The out-of-plane bending term (the fourth term of the force-field equation) describes the energy required to achieve correct geometrical alignment of atoms. For example, without the out-of-plane term, the oxygen atom in cyclobutanone $\left(\mathrm{C}_{4} \mathrm{H}_{6} \mathrm{O}\right)$ is incorrectly predicted to lie out of the plane of the ring, adopting a valence angle close to its reference value of $120^{\circ}$. Experimentally, the oxygen atom should lie in the plane of the cyclobutane ring. The inclusion of the out-of-plane term ensures the correct alignment is achieved during equilibration. In equation (2.2), the out-of-plane bending term is characterized by the angle between the plane of the molecule and the out-ofplane bond $(\xi)$. A value of $0^{\circ}$ corresponds to the atom being in plane.

Terms 5-10 in equation (2.2) are referred to as cross terms, and describe the coupling interaction between internal coordinates i.e., they reflect the change of valence and torsional angles a well as bond lengths due to neighboring distortions. For example, as a valence angle is decreased it is found that the adjacent bonds stretch to reduce the increased interaction caused by the angle bending. Cross terms are often required to 
achieve optimal performance and specifically to predict vibrational frequencies and structural variations associated with conformational changes. The force-field chosen in this study comprises six cross terms that are a function of two internal coordinates, and one cross term that is a function of three internal coordinates. These are: stretch-stretch (term 5), stretch-bend (term 6), stretch-torsion (term 7), bend-torsion (term 8), bendbend (term 9) and bend-bend-torsion (term 10).

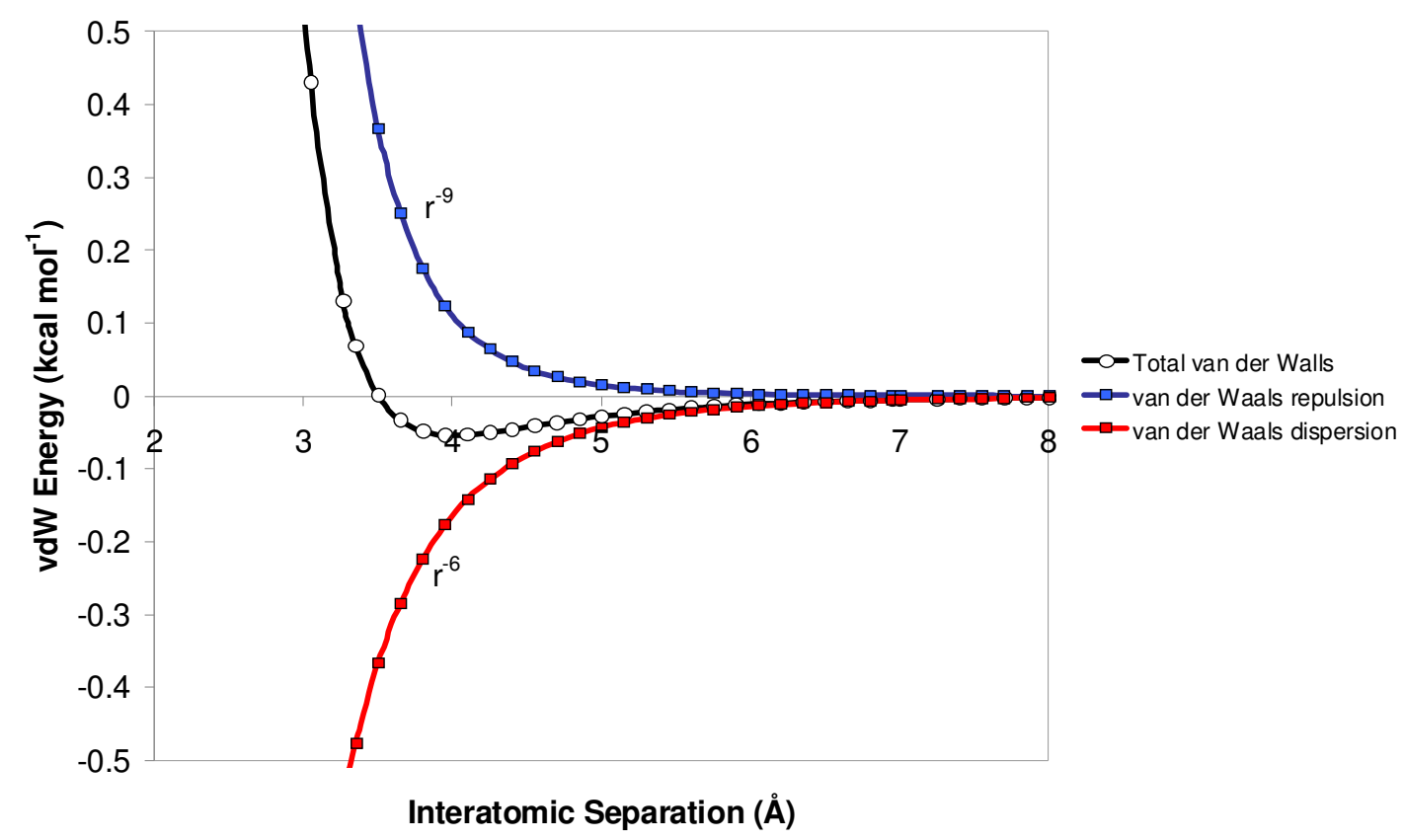

Figure 2.1 Lennard Jones 9-6 potential for two isolated carbon atoms, comprising of a repulsive component, varying as the inverse ninth power of the interatomic distance, and an attractive component, varying as the inverse twelfth power of the distance.

The non-bonded terms (electrostatic and van der Waals) are usually represented mathematically as two-body interactions. In equation (2.2), the electrostatic interaction (term 11) is modeled using Coloumb's law, where $q_{i}$ and $q_{j}$ are the point charges of atoms $i$ and $j$ respectively, and $r_{i j}$ is the inter-atomic distance. The charge $q$ is restricted to the centre of each atom and its assigned value chosen carefully to reproduce the electrostatic properties of the molecule/atomic environment. It represents the partial atomic charge of the atom.

The van der Waals (vdW) interaction energy is described by term 12, and modelled using the Lennard Jones 9-6 potential. An example of a graphical representation of this 
potential for two isolated carbon atoms is shown in Figure 2.1. It can be seen from the graph that the vdW potential approaches zero at an infinite inter-atomic separation. As the separation is reduced, the energy decreases passing through a minimum. The energy then rapidly increases as the separation decreases further. The vdW interaction arises from a combination of attractive and repulsive forces which are also shown in Figure 2.1 by the red and blue lines respectively. The attractive forces are referred to as dispersive interactions and are long range acting. These dispersive forces arise due to instantaneous dipoles that occur during fluctuations in the electron clouds. Even if the molecule has no permanent dipole moment, the motion of the electrons will create a slightly uneven distribution at a given time. The dipole moment will induce a charge polarization in the neighboring particle, creating an attraction that varies as the inverse sixth power of the distance between the two particles. The induced dipole-dipole interaction is the dominant dispersive interaction however there are other contributions to the dispersive force, such as induced dipole-quadrupole and quadrupole- quadrupole, which are not considered in the expression of the vdW potential. However, the expression used in term 12 , which varies as the inverse sixth power of the distance separating the particles, is shown to agree well with experiment, as it successfully describes the attractive contribution to the vdW interaction. ${ }^{117}$ The repulsive forces due to $\mathrm{vdW}$ interactions arise from the fact that Pauli exclusion principle prohibits any two electrons in the same orbital or internuclear region from having the same spin. Therefore when two nonbonding atoms are brought close to each other, there is a reduced electron density in the internuclear region. This results in repulsion between the incompletely shielded nuclei. While this repulsive interaction is most commonly modeled by a form that varies as the inverse twelfth power of the inter-atomic distance, such a form is often considered to be too 'hard' in the repulsive region and so a 'softer' potential that varies as the inverse ninth power of the distance is used in COMPASS. ${ }^{118}$

The functional form of the COMPASS force-field does not contain explicit representation of hydrogen bonds. It considers these within its van der Waals and electrostatic parameters for an improved force-field fit to experimental data. ${ }^{73}$ 


\subsubsection{Force-Field Parameterization}

The foundation of the force-field method is that the units that constitute a molecule can be structurally similar in different molecules. For example, all $\mathrm{C}-\mathrm{H}$ bonds exhibit a similar length, in the range of 1.06-1.10 $\AA$. The C-H vibrations are also similar (2900$3300 \mathrm{~cm}^{-1}$ ) across different molecules. If the $\mathrm{C}-\mathrm{H}$ bonds are further divided into groups, with those attributed to single-, double-, or triple-bonded carbon, the structural variation within each of these groups becomes even smaller. In force-field methods, the idea of atoms being structurally similar in different molecules is implemented through different atom types. Each atom type is assigned its own set of parameter values. The atom type depends on the atomic element and its chemical environment i.e., the chemical bonding it is involved in, and denoted with either a number or a letter code. For example, in the COMPASS force-feld, ${ }^{73}$ a hydrogen that is bonded to nitrogen is denoted by $\mathrm{h} 1 \mathrm{n}$.

In a force-field calculation, each atom is assigned its own set of parameter values, which describe the chemical or physical bonding that the particular 'atom type' is involved in. Force-field parameterization refers to the calculation of these parameter values. It involves none other, than assigning numerical values to the constants of the force-field energy expression of eq. (2.2). Force-field parameters can be derived by fitting to experimental data, empirical parameterization, and/or from electronic structure calculations, ab-initio parameterization.

Parameterization of the COMPASS force-field, was undertaken by $\operatorname{Sun}^{73}$ and involved a two stage hybrid procedure. In the first stage, partial charges and valence parameters (e.g. bond length, spring constants) were derived by fitting to ab-initio data. The atomic partial charges were derived based on ab-initio calculation of the electrostatic potential energy (ESP). The ESP is the potential arising from the force that is acting on a unit of positive charge. The valence parameters were determined from abinitio energies including first and second derivative of the total energies. During this first stage, the vdW parameters were fixed to a set of initial values taken from the polymer consistent force-field (PCFF). In the second stage of the procedure, empirical optimization was undertaken where emphasis was on refining the values of the parameters to yield good agreement with experimental data. During empirical optimization, the valence parameters were adjusted based on the following properties: 
molecular structures (bond lengths and bond angles), molecular dipole moments, vibrational frequencies, and conformational energies. Refinement of valence parameters was performed on isolated molecules i.e., molecules in their gas phase. The vdW parameters were also adjusted based on density and cohesive energy values of molecular liquids i.e., molecules in their condensed phase.

A critical feature of a general force-field is that its parameters are compatible among the different functional groups. The concept is referred to as transferability and the COMPASS force-field has been parameterized for a wide range of organic compounds such as alkanes and esters, but also for inorganic molecules and materials. In the interest of this study, the COMPASS force-field has been used to model condensed phase polymeric system and tested for a wide range of organic / inorganic interfaces. ${ }^{76,95,116,119}$ Further details of the parameterization of the COMPASS force-field can be found in the study of Sun. ${ }^{73}$

\subsection{Energy Minimisation}

The term 'energy surface' is often used to describe the way in which the potential energy of the system varies as a function of its coordinates. For a large system of $N$ number of atoms, the energy surface can be rather complex, as the potential will be a function of $3 \mathrm{~N}$ Cartesian coordinates. One of the most interesting aspects of the energy surface is the minimum energy points, as these correspond to stable states of the system. There may be a very large number of minima on the energy surface, and the one with the lowest energy is known as the global energy minimum. To attain a configuration that corresponds to a closest energy minimum, an algorithm known as energy minimisation is used. ${ }^{120,121}$ Another way to look at this, is that energy minimisation results in minimum potential configuration attained by the most efficient movement of the atoms in the system.

The derivative of the energy function with respect to the atomic coordinates, the energy gradient, provides important information useful in the energy minimisation process. In particular, the direction of the gradient indicates where a minimum is located, and the magnitude of the gradient indicates how steep the energy slope is. The energy of the system is then reduced by stepping in the negative direction of the gradient (i.e. the direction of the net force). The energy eventually reaches a minimum when the first 
derivative is zero and the second derivative is positive. There is a vast number of energy minimisation methods available. ${ }^{120,121}$ First order methods use the first derivatives of the potential whereas second order methods use both first and second derivates. In some cases, non-derivative methods may also be used. In this study, the first order derivative minimisation methods of steepest descent and conjugate gradient are used.

In these methods, steps are taken in the negative direction of the energy gradient. Iterations are then made in this path, to locate an energy point that is lower in potential than the previous point. This process is commonly referred to as a line search. Once the point of lowered energy is located, the next direction in the algorithm is determined. In the steepest descent method, this direction is always perpendicular to the previous direction, whilst in the conjugate gradient method the direction is the conjugate of the previous direction. The local energy minimum is eventually attained when the convergence criterion is met. While the steepest descent is robust and ideal for quickly relieving high energy strains in the system, it forces a right angle turn at each point, even though this may not be the best route to the local energy minimum. At times this can result in an unnecessary number of steps being performed. In the case of the conjugate gradient method, its route does not exhibit the oscillatory behaviour of the steepest descent method. For this reason, when stringent minimisation and high convergence criterion is required, the conjugate gradient method is often a preferred option, as the local energy minimum can be attained in far fewer steps.

\subsection{Molecular Dynamics}

To simulate the motion of atoms in a system and investigate its structural evolution, the molecular dynamics approach is employed. In molecular dynamics, the system is allowed to evolve at a finite temperature according to the Newton's second law of motion:

$$
\mathbf{F}=-\frac{d V}{d \mathbf{r}}=m \frac{d^{2} \mathbf{r}}{d t^{2}}=m \mathbf{a}
$$

Here $V$ is the potential energy determined by the force-field energy expression of eq. (2.2), $\mathbf{r}$ is the vector that contains $\mathrm{x}, \mathrm{y}$ and $\mathrm{z}$ coordinates of the particles, and $\mathbf{F}$ and $\mathbf{a}$ are the force and acceleration vectors respectively. To begin a dynamics simulation, we 
require a set of initial coordinates and velocities, and of course the interaction potential. For a short period of time, the interaction within the system may be considered constant. This period is known as the time step, during which the interaction between atoms are computed and combined with the current position and velocities to generate new atomic positions and velocities. The atoms are then moved to their new position, and a set of updated positions and velocities are determined. By undertaking a large number of time steps, a molecular dynamic trajectory is generated, and the time behavior of the system is obtained.

In comparison to the energy minimisation process, molecular dynamics (MD) allows for a larger portion of the energy surface to be explored. Also, MD allows for time dependent properties to be determined, since atomic movement is generated according to Newton's second law of motion. There are many example of where MD has been used to probe the dynamics of many organic and inorganic interfaces, including those of interest to this work. ${ }^{75,76,94,97,98,115,122-124}$

There are a number of algorithms used to numerically solve Newton's equation of motion and determine new positions and velocities. In this study, the velocity Verlet algorithm $^{125}$ is used which is a variant of the Verlet method. ${ }^{126}$ In the velocity Verlet algorithm the position (r) and velocity ( $\mathbf{v})$ vectors after time step $\Delta t$ are defined as follows:

$$
\begin{gathered}
\mathbf{r}_{(t+\Delta t)}=\mathbf{r}_{(t)}+\mathbf{v}_{(t)}(\Delta t)+\frac{1}{2} \mathbf{a}_{(t)}(\Delta t)^{2} \\
\mathbf{v}_{(t+\Delta t)}=\mathbf{v}_{(t)}+\frac{1}{2} \Delta t\left(\mathbf{a}_{(t)}+\mathbf{a}_{(t+\Delta t)}\right)
\end{gathered}
$$

The position (equation (2.4)) is evaluated as a Taylor series expansion. To calculate the new velocity $\mathbf{v}_{(t+\Delta t)}$ from equation (2.5), values of the acceleration at times $(t)$ and $(t+$ $\Delta t)$ are required concurrently $\left(\mathbf{a}_{(t)}\right.$ and $\left.\mathbf{a}_{(t+\Delta t)}\right)$. Therefore, the velocity Verlet method is implemented as a three stage process. Firstly, the new position vector $\mathbf{r}_{(t+\Delta t)}$, is determined according to the equation (2.4). In this equation, the initial values of the velocities $\mathbf{v}_{(t)}$ are randomly chosen from a Maxwell-Boltzmann distribution, and the acceleration $\mathbf{a}_{(t)}$, determined from the derivative of the potential (equation (2.3)). 
Secondly, the atomic positions are propagated to their new coordinates and again with the use of the interaction potential (equation (2.3)), the new values of the acceleration are determined $\left(\mathbf{a}_{(t+\Delta t)}\right)$. Finally, values of $\mathbf{a}_{(t)}$ and $\mathbf{a}_{(t+\Delta t)}$ are used to determine the new velocity vector $\mathbf{v}_{(t+\Delta t)}$. The velocity Verlet algorithm assumes that the acceleration is dependent on the position of the particles and not on their velocities.

\subsection{Periodic Boundary Conditions}

A system's size is required to be large enough so that the macroscopic properties calculated from simulation match those of experiment. Periodic boundary conditions ${ }^{120,121}$ in theory allow for a system of "infinite" size to be modelled. Firstly, particles are enclosed in a box known as a unit cell. This unit cell therefore contains all "unique" atoms of the system. This cell is then replicated by rigid translation in all three Cartesian directions ( $\mathrm{x} y$, and $\mathrm{z}$ ) forming an infinite sized system. For example, assuming a particle is positioned at vector $\mathbf{r}$ in a unit cell, when periodic boundary conditions are applied, the particle is replicated infinitely in the following manner;

$$
\mathbf{r}_{\text {image }}=\mathbf{r}+l \mathbf{a}+m \mathbf{b}+n \mathbf{c}
$$

where $l, m$ and $n$, are integers ranging from $-\infty$ to $+\infty$, and $\mathbf{a}, \mathbf{b}$ and $\mathbf{c}$ are the vectors corresponding to the unit cell. The vector $\mathbf{r}_{\text {image }}$ from Equation (2.6), represents the coordinates of the "image particles". Effectively, each particle is now considered to be interacting not only with other particles within the unit cell, but also with image particles in neighbouring boxes. If a particle leaves the unit cell during molecular dynamic simulations, it will be replaced by an image particle that enters from the opposite side. The number of particles within the unit cells thus remains constant. The use of such periodic boundary conditions also eliminates surface effects near boundaries which can lead to erroneous results. This is in fact the greatest advantage of implementing periodic boundary conditions.

\subsection{Truncation of Non-Bonded Interactions}

One of the most important aspects of a molecular mechanics or dynamics simulation is the calculation of the non-bonded interactions that arise from electrostatic and vdW 
forces. ${ }^{120,121}$ The evaluation of these interactions can be computationally expensive and time consuming because of the large number of pair-wise interactions that need to be accounted for. In comparison, bonded interactions are far less computationally demanding to evaluate. For example, in a single linear alkane chain $\mathrm{CH}_{3}\left(\mathrm{CH}_{2}\right)_{18} \mathrm{CH}_{3}$, the non-bonded contributions alone account for $83 \%$ of computational effort. The greatest contribution to $\mathrm{vdW}$ and electrostatic interactions arises from neighbouring particles, and it is for this reason, that long-range non-bonded interactions are often neglected or approximated. One common method that can be used to reduce the computational cost associated with evaluation of non-bond interactions is to apply a minimum image convention. In this method, the non-bonded interactions are computed based on the fact the each particle interacts with the closest image of the remaining particles in the system. In addition, an atom based cut-off may be implemented, whereby non-bonded interactions are evaluated to a specific inter-atomic separation known as the cut-off distance. Beyond this point non-bonded interactions are neglected.

The introduction of a cut-off distance alone does not lead to a significant computational saving, since in theory all inter-atomic distances must be computed prior to the decision on whether to include the contribution or not. A further increase in computational efficiency can by achieved during molecular dynamics (MD) by introducing a nearest neighbor list of atom pairs. From a given starting geometry, a list is prepared comprising of atom pairs that are separated within the cut-off distance plus a small buffer zone or buffer width. When the energy is evaluated according to the forcefield expression of eq. (2.2)), only the non-bonded interactions from atom pairs on the list are evaluated, which avoids calculation between all pairs of atoms. When the geometry of the system changes during MD, the non-bonded list must be updated. However, the list is not updated after every step, i.e. in time step intervals, but updated after a suitable time interval which allows atoms to move more than the buffer width.

When atom based cut-off methods are implemented, it is vital that contributions larger the cut-off distance are not neglected. This is because the energy function can otherwise become discontinuous, which may cause problems in energy minimisation and molecular dynamic simulations. Often, a long range correction or smoothing function is employed to alleviate such effects by smoothly 'turning off' interactions between atoms 
separated by a distance larger than the cut-off criteria. Typically, smoothing functions are in the form of polynomials and attempt to correctly connect the vdW and electrostatic interaction energy to zero.

An alternative method to the atom based cut-off approach is the Ewald summation technique. ${ }^{127}$ The Ewald approach provides a decaying potential which results in rapid convergence of the non-bonded interactions (electrostatic and $\mathrm{vdW}$ ) at long range. The main principle behind this method involves multiplying the non-bonded energy expression by a convergence function. The resulting expression then becomes a sum of two terms. The first term, represents the short range contributions that converge rapidly as a result of the inclusion of the convergence function. The second term represents the long-range contributions which converge very slowly. However, by taking the Fourier transform of the second term, the resulting expression converges much faster. How fast this potential decays is governed by the convergence parameter set by the user.

\subsection{Thermodynamic Ensembles}

An ensemble is a collection of a large number of systems, each constructed to replicate the thermodynamics state or 'environment' of the system whose properties we are interested in. ${ }^{128}$ In this study, two ensembles are employed; the canonical ensemble, and the isothermal-isobaric ensemble. In the canonical ensemble the thermodynamic state of the system of interest is described by a fixed volume $\mathrm{V}$, fixed number of molecules $\mathrm{N}$, and fixed temperature $\mathrm{T}$. Therefore, during molecular dynamics in the canonical ensemble (NVT), the system under periodic boundary conditions is evolved at constant volume and temperature. ${ }^{120,121}$ Alternatively, for molecular dynamics in the isothermal-isobaric ensemble (NPT), the pressure and temperature are kept fixed. This NPT ensemble is important in obtaining a correct density of the system. Details of the algorithms used to control the temperature and pressure are provided in Sections 2.9 and 2.10 respectively. 


\subsection{Controlling the Temperature}

The temperature $(T)$ is an important concept in a molecular dynamics simulation, as it specifies the thermodynamic state of the system. It is related to the time average of the kinetic energy $\langle K\rangle$;

$$
\langle K\rangle=\left\langle\sum_{i=1}^{N} \frac{m_{i} v_{i}^{2}}{2}\right\rangle=\frac{3}{2} N_{f} k_{B} T
$$

where $N$ is the number of particles in the system, $N_{f}$ is the number of degrees of freedom, $m$ and $v$ are the mass and velocities of the particles respectively, and $k_{B}$ is the Boltzmann constant. In a molecular dynamics simulation, the initial velocities are generated so as to produce a Maxwell-Boltzmann distribution at the desired temperature. However as the simulation continues, the temperature does not remain constant since kinetic and potential energy are exchanged. To maintain a constant temperature, the velocities need to be adjusted accordingly. In the direct velocity scaling approach ${ }^{129}$ the target temperature is maintained, by simply multiplying the velocities by a factor $\lambda$ corresponding to the desired temperature change. In the Berendsen approach, ${ }^{130}$ the multiplication factor $\lambda$ is associated with the heat-exchange between the system and a heat bath. The Berendsen scaling method is a more gentle approach compared to direct velocity scaling, as the velocities are scaled such that the rate of change of temperature is proportional to the difference in temperature between the bath and the system. Nevertheless, both scaling methods do not generate rigorous canonical averages whereby the probability that a configuration of energy $E$, is proportional to the Boltzmann factor. An alternative method to velocity scaling that generates rigorous canonical ensembles is the Andersen temperature control method. ${ }^{131}$ This method is based on stochastic collisions between particles. The velocities of the particles are adjusted to produce a predefined collision frequency. Effectively, a series of constant energy states are generated whose distribution of energies match a Gaussian function. In this work, the Andersen thermostat has been used to control the temperature in the canonical (NVT) and isothermal-isobaric (NPT) ensembles. 


\subsection{Controlling the Pressure}

Simulations in the isothermal-isobaric ensemble NPT, are most relevant to experimental conditions, because most experiments are carried out at constant temperature and pressure. To maintain a system at constant pressure, the pressure needs to be adjusted by changing the volume of the simulation cell. The magnitude of the volume change $(\partial V)$ is related to the change in pressure $(\partial P)$ via the isothermal compressibility $(\kappa)$;

$$
\kappa=-\frac{1}{V}\left(\frac{\partial V}{\partial P}\right)_{T}
$$

A substance of high compressibility exhibits a large volume change at fixed pressure. Many of the methods used for pressure control are analogues to those used for temperature control. In this work we employ the Berendsen barostat ${ }^{130}$ to control the pressure during simulations in the isothermal-isobaric ensemble. The pressure is maintained at a constant value by multiplying the volume of the cell by a scale factor $(\lambda)$. However in practice it is actually the atomic coordinates which are scaled by a factor $\lambda^{1 / 3}$. The scale factor is defined by the following equation;

$$
\lambda=1-\kappa \frac{\delta t}{\tau_{p}}\left(P_{b a t h}-P(t)\right)
$$

where $\tau_{p}$ is the coupling constant, and $P$ and $P_{b a t h}$ are the pressure values of the system and bath, respectively. The constant $\kappa$ is often combined with the relaxation constant $\tau_{p}$ as a single constant. 


\section{Chapter 3}

\section{Molecular Models and Theoretical Procedures}

\subsection{Overview}

One of the major challenges in using computer simulations to model materials and gain accurate insight into the mechanics or dynamics of the system is the construction of the models themselves. In this section, we will present the models that were carefully selected to emulate realistic paint coatings and commonly encountered atmospheric dirt particulates. We will describe the various coating modifications that were undertaken to test the resistance of the surface against contaminant adhesion. These modifications were inspired by none other than the natural self-cleaning ability of plant leaves. Finally, we will examine the method used to calculate the strength of adhesion between coating and contaminant. 


\subsection{Polyester Surface Models}

The surface coating was simulated using a cured polyester model based on the work of Yarovsky et al. ${ }^{94}$ The chemical structure of the monomeric units of a single polyester resin molecule, are shown in Figure 3.1. A single resin molecule is composed of 15 BEPD (2-Butyl-2ethyl-1,3-propanediol), 2 TMP (Trimethylolpropane) and 16 IPA (Isophthalic acid) repeat units. Three polyester resin molecules were combined with butoxymethyl melamine crosslinker to create a model of a cured top-coat with a resin:crosslinker molecular weight ratio of 80:20. The density of the polymer model $\left(1.14 \mathrm{~g} \mathrm{~cm}^{-3}\right)$ was adopted from experiment. The polyester was packed in a periodic unit cell of dimensions of $40 \times 40 \times \sim 15 \AA$, exhibiting a surface area of $16 \mathrm{~nm}^{2}$. The total number of atoms in the polymer unit cell was equal to 2454. A detailed description of the procedure used to construct the model is described in the theoretical study of Yarovsky et al. ${ }^{94}$<smiles>CCCCC(CC)(CO)CO</smiles>

$$
\begin{aligned}
& \text { 2-Butyl-2-ethyl-1,3-propenediol } \\
& \text { (BEPD) } \\
& \quad \mathrm{C}_{9} \mathrm{H}_{20} \mathrm{O}_{2} \\
& \text { Mol. Wt.: } 160.2558
\end{aligned}
$$

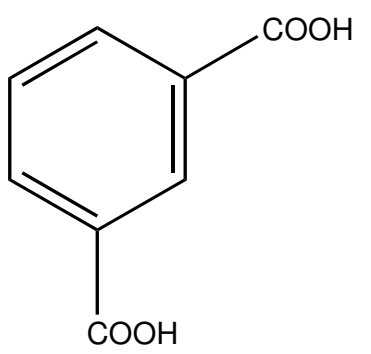

Isophthalic acid (IPA)<smiles>CCC(CO)(CO)CO</smiles>

Trimethylolpropane (TMP)

$$
\mathrm{C}_{6} \mathrm{H}_{14} \mathrm{O}_{3}
$$

Mol. Wt.: 134.1748

Figure 3.1 Chemical composition of the monomeric units used to construct the polyester coating model. 


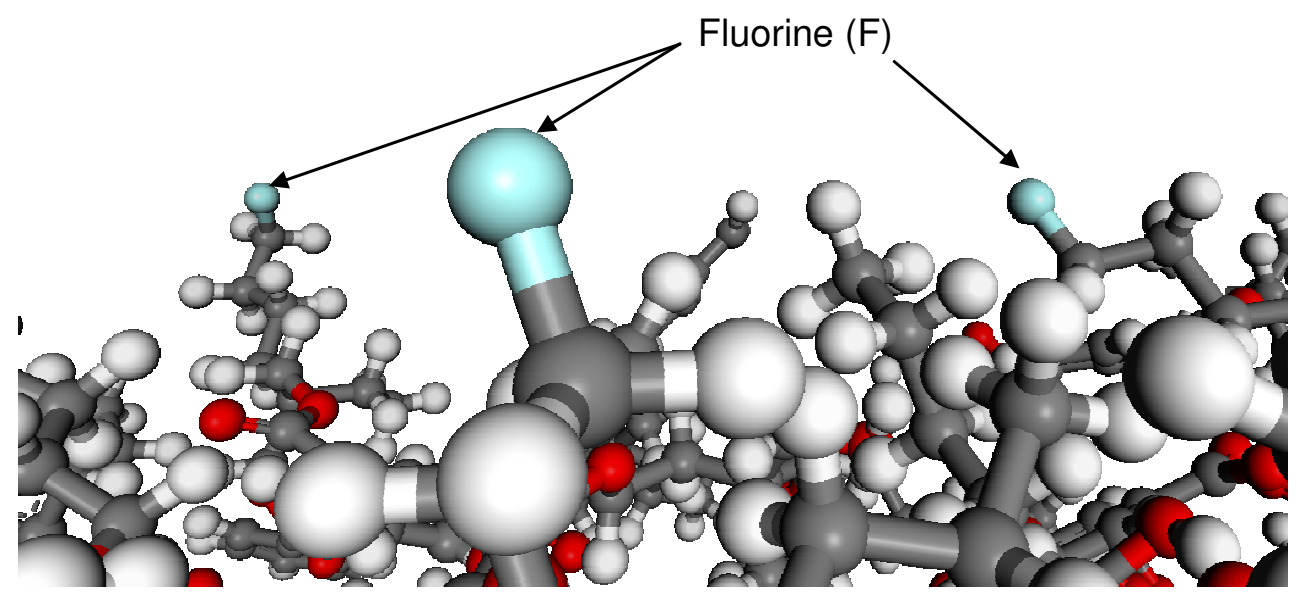

Figure 3.2 Model of a nano-scale modified surface coating. Surface modifications are undertaken by replacement of hydrogen atoms, with fluorine $(\mathrm{F})$, hydroxyl $(\mathrm{OH})$ or carboxyl $\left(\mathrm{CO}_{2} \mathrm{H}\right)$ groups. In this figure, the hydrogen, fluorine, oxygen and carbon atoms are represented by white, light blue, red and grey beads respectively. The fluorine modifications are employed to improve the carbon-shielding ability of the coating.

Modified polyester surfaces denoted PolyesternX were constructed by substituting the protruding hydrogen atoms along the polyester surface, with fluorine $(X=F)$, carboxyl $\left(\mathrm{X}=\mathrm{CO}_{2} \mathrm{H}\right)$, and hydroxyl $(\mathrm{X}=\mathrm{OH})$ groups. A schematic illustration of a fluorine modified polyester is shown in Figure 3.2. The protruding hydrogen atoms were selected randomly by eye from the visualizer.

While fluorine modification was undertaken to mimic hydrophobic Teflon-like surfaces, ${ }^{37,49,50}$ hydroxyl and carboxyl modifications were used to improve the film's hydrophilicity in accordance with UV/ozone treatments. ${ }^{132-134}$ Different levels of modification were investigated by varying the number $(n)$ of functional groups on the surface of the polyester. The introduction of 7, 15, and 75 functional groups per unit cell achieved a surface coverage of $0.4,0.9$, and 4.7 residues per $\mathrm{nm}^{2}$, respectively. Modified polyester surfaces Polyester7X and Polyester15X were constructed by replacing one hydrogen atom in each of the 7 and 15 most accessible methyl groups, respectively. The accessible methyl groups were selected randomly by eye. Two types of modified polyesters with a surface coverage of 4.7 residues per $\mathrm{nm}^{2}$ were prepared (Polyester(25x3)X and Polyester75X), that is, the same quantity of functional groups (75) was introduced to the surfaces of these systems, but their distribution across the surface varied. For the Polyester(25x3)X systems, three hydrogen atoms in each of the 
25 most accessible methyl groups of the surface were replaced with fluorine or hydroxyl groups. This modification produced surfaces in which a moderate number $\left(1.6\right.$ per $\left.\mathrm{nm}^{2}\right)$ of perfluorinated and perhydroxylated methyl groups have been formed on the surface. Alternatively, for Polyester75X systems, the 75 most accessible hydrogens were substituted with $\mathrm{F}$ (Polyester75F) or $\mathrm{OH}$ (Polyester75OH) groups, regardless of their chemical environment. Therefore, in the Polyester $75 \mathrm{X}$ systems, the functional groups are distributed more uniformly across the surface (i.e., they are less localized than for Polyester(25x3)X). Each modified polyester surface was subsequently energy minimised. A summary of the polyester surfaces examined is presented in Table 3.1.

Table 3.1 Polyester Surfaces Examined

\begin{tabular}{|c|c|c|}
\hline & surface, $N$ & surface modelled \\
\hline 1 & polyester & unmodified polyester \\
\hline 2 & Polyester7F & fluorinated polyester $\left(0.4 \mathrm{~F} \mathrm{~nm}^{-2}\right)$ \\
\hline 3 & Polyester7OH & hydroxylated polyester $\left(0.4 \mathrm{OH} \mathrm{nm}^{-2}\right)$ \\
\hline 4 & Polyester7 $\mathrm{CO}_{2} \mathrm{H}$ & carboxylated polyester $\left(0.4 \mathrm{CO}_{2} \mathrm{H}\right.$ per $\left.\mathrm{nm}^{-2}\right)$ \\
\hline 5 & Polyester15F & fluorinated polyester $\left(0.9 \mathrm{~F}\right.$ per $\left.\mathrm{nm}^{-2}\right)$ \\
\hline 6 & Polyester $15 \mathrm{OH}$ & hydroxylated polyester $\left(0.9 \mathrm{OH}\right.$ per $\left.\mathrm{nm}^{-2}\right)$ \\
\hline 7 & Polyester $15 \mathrm{CO}_{2} \mathrm{H}$ & carboxylated polyester $\left(0.9 \mathrm{CO}_{2} \mathrm{H} \mathrm{nm}^{-2}\right)$ \\
\hline 8 & Polyester(25x3)F & fluorinated polyester $\left(4.7 \mathrm{~F} \mathrm{~nm}^{-2}, 1.6 \mathrm{CF}_{3}\right.$ per $\left.\mathrm{nm}^{-2}\right)$ \\
\hline 9 & Polyester $(25 \times 3) \mathrm{OH}$ & hydroxylated polyester $\left(4.7 \mathrm{OH} \mathrm{nm}^{-2}, 1.6 \mathrm{C}(\mathrm{OH})_{3} \mathrm{~nm}^{-2}\right)$ \\
\hline 10 & Polyester75F & fluorinated polyester $\left(4.7 \mathrm{~F} \mathrm{~nm}^{-2}\right)$ \\
\hline 11 & Polyester75OH & hydroxylated polyester $\left(4.7 \mathrm{OH} \mathrm{nm}^{-2}\right)$ \\
\hline
\end{tabular}

\subsection{Models of Carbon Contaminants}

Atmospheric carbonaceous particles are often the product of biomass and fossil fuel burning. A range of particle types, with distinct properties, can result depending on the nature of the fuel and the combustion conditions. For example, soot particles are 
spherical with diameters of 20 to $30 \mathrm{~nm}$ and comprise of an onion-shell structure composed of approximately 7 to 10 graphitic $\left(\begin{array}{lll}0 & 0 & 1\end{array}\right)$ planes, often surrounding an amorphous core. ${ }^{135-137}$ In comparison tar balls are amorphous carbonaceous spherules with diameters typically between 30 and $500 \mathrm{~nm}$ distinguished from soot by their morphology and lack of turbostratic microstructure. ${ }^{3}$ For this investigation, three carbon models are used to simulate the contaminant particles and surfaces: graphite, amorphous carbon $(\mathrm{AmCH})$ and fullerene $\left(\mathrm{C}_{60}\right)$. These carbon models are illustrated in Figure 3.3.

\section{a. Graphite}

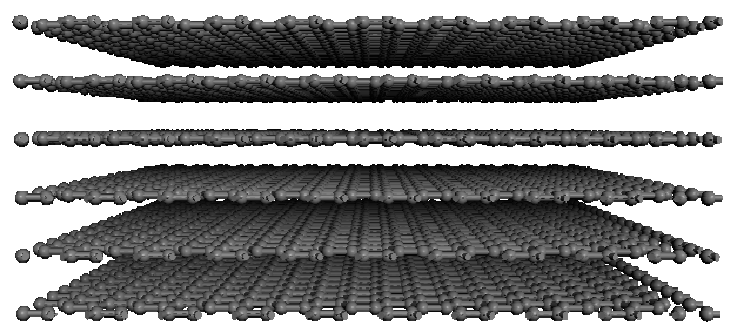

c. Fullerene, $\mathrm{C}_{60}$

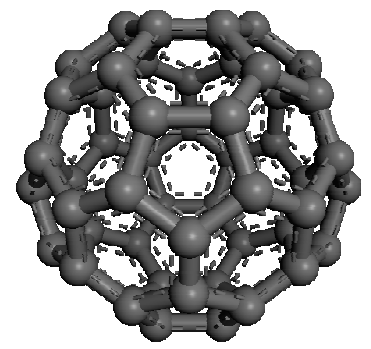

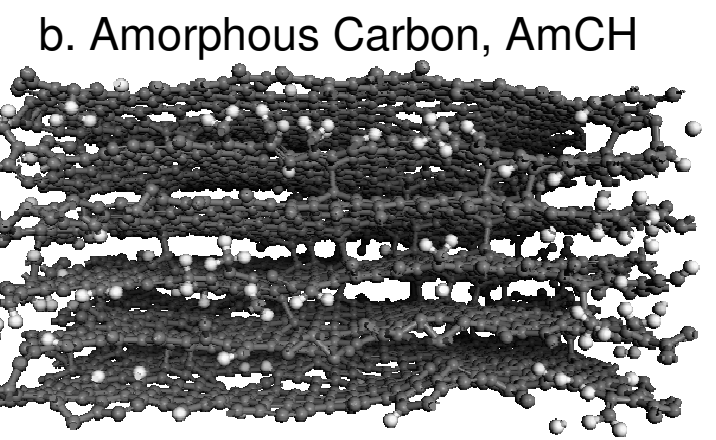

Figure 3.3 Contaminant models. a, Graphite represents the surface of an ideal soot particle. b, The Amorphous Carbon (AmCH) model is based on realistic coal derived contaminant and exhibits an irregular surface morphology analogous to tar-balls. $c$, The fullerene $C_{60}$ model represents a soot particulate.

The graphite model, comprising of six ideally flat graphitic (001) layers ${ }^{138}$ is used to model an ideal soot-particle surface. ${ }^{135-137}$ The average spacing between sheets is $3.5 \AA$. The constituent atoms of graphite are $\mathrm{sp}^{2}$ hybridized carbon atoms and to maintain overall neutrality they do not carry any partial charge. The graphite model is packed in a unit cell of dimensions of 40 x 40 x $17 \AA$, exhibiting a surface area of $16 \mathrm{~nm}^{2}$.

The amorphous carbon model $(\mathrm{AmCH})$ used in this study is comprised of six disordered and buckled graphite-like sheets composed mainly of aromatic carbon atoms, 
with some interstitial bonding by $\mathrm{sp}^{3}$ hybridized carbons. The model exhibits an average inter-layer spacing of $3.5 \AA$ as in the case of graphite. It is based on a previously constructed 3-D periodic model of an amorphous industrial char. ${ }^{139}$ This model was generated via Hybrid Reverse Monte Carlo (HRMC) simulations ${ }^{140}$ using experimental pair correlation functions obtained via electron diffraction techniques. ${ }^{141}$ The amorphous carbon model is therefore consistent on a nano-scale with the surface of a realistic coalderived carbonaceous particle. The model was subsequently resized to $40 \AA \times 40 \AA \times$ $\sim 20 \AA$, so that its dimensions are comparable to that of the polyester surface models and hydrogenated where necessary. The final structure which exhibits a surface area of 16 $\mathrm{nm}^{2}$ is referred to as hydrogenated amorphous carbon $(\mathrm{AmCH})$. In contrast to the graphite model, the amorphous carbon surface is structurally irregular, and its constituent atoms carry partial atomic charges.

The $\mathrm{C}_{60}$ fullerene model exhibits a spherical graphitic like structure and therefore provides a convenient model of a soot particulate. In a similar manner to graphite, the $\mathrm{C}_{60}$ model comprises of $\mathrm{sp}^{2}$ hybridized carbon atoms, which do not carry any partial atomic charge. The surface area of the model is approximately $3 \mathrm{~nm}^{2}$.

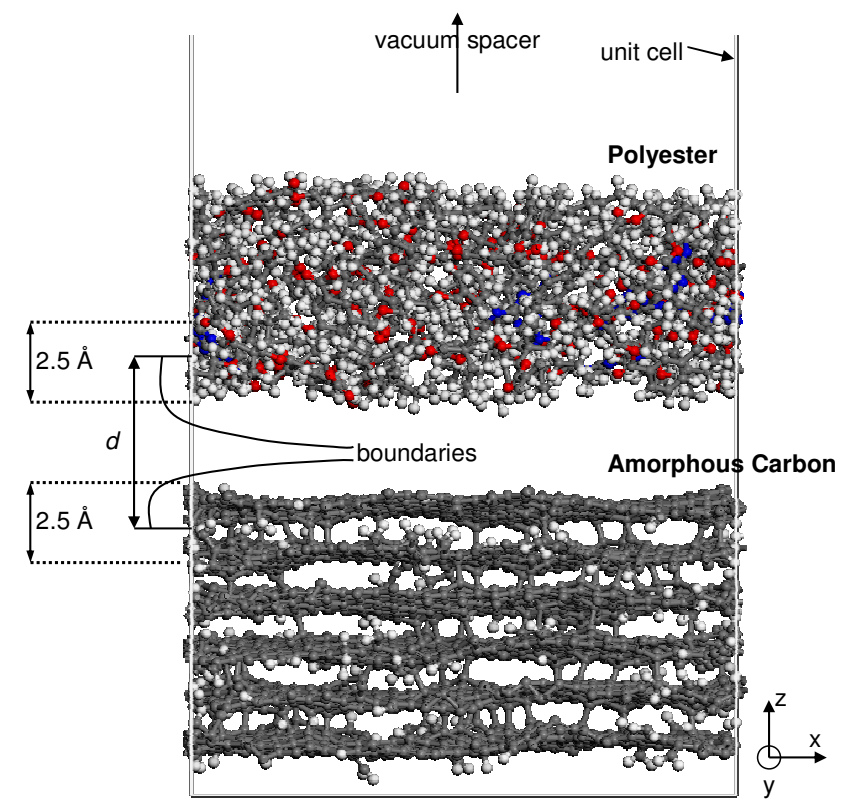

Figure 3.4 A schematic representation of the polyester/amorphous carbon interface, and the interfacial separation $d$. This interface represents a carbon contaminant interacting with a polyester surface coating. The interfacial separation is the distance between the boundaries of the polymer and carbon models. The boundaries were determined by averaging the $z$ positions of atoms in the leading $2.5 \AA$ of the materials. 


\subsection{Modelling Adhesion}

Having chosen appropriate models to represent the surface coatings and contaminant particles, we employed a procedure to evaluate the interaction between the polymer surface and carbon contaminant. The procedure involves firstly forming a polymer / carbon interface by combining a single polymer surfaces with each carbon model in a periodic unit cell. An example of this is shown in Figure 3.4 (previous page), illustrating a polyester coating interacting with the amorphous carbon contaminant model in a unit cell. As shown in this figure, the unit cell was extended in the $\mathrm{z}$ direction to ensure that adjacent systems do not interact along the $z$ axis when 3-D periodic boundary conditions are imposed. When these conditions are imposed, the system mimics a 2-D interface along the $\mathrm{x}$ and $\mathrm{y}$ dimensions.

Interfaces were formed both in vacuum and in aqueous environment, and all contaminant models tested. The methods used to probe these interfaces varied slightly, depending on the system in question. These methods are presented in detail in the following chapters. Here however, we present a basic method to determine the strength of adhesion between two infinite surfaces interacting in vacuum. The method involves evaluating the total energy of the system as a function of the separation between surfaces $(E(d))$. Specifically, we monitor the energy as the isolated surfaces are brought into close contact to form the interface or alternatively as the interface is separated into two free surfaces. ${ }^{142}$ The adhesion energy $\left(E_{a d h}(d)\right)$ is obtained from the relationship

$$
E_{a d h}(d)=\frac{E(d)-E(\infty)}{A}
$$

where $E(\infty)$ is the total energy at infinite separation, and $A$ is the cross-sectional area of the interface. A plot of adhesion energy as a function of interfacial separation (Figure 3.5) enables us to determine interfacial properties that characterise the adhesion between surfaces. The most important property is the work of separation $\left(W_{\text {sep }}\right)$, calculated by the depth of the potential well of the adhesion curve. The work of separation, as defined by Finnis, ${ }^{143}$ is the work required to separate an interface instantaneously into two free surfaces. This property is related to non-bonded interactions (van der Waals and electrostatic) but does not take into account additional energy due to diffusion, surface 
segregation, relaxation, and/or reconstruction during cleavage of the interface. In practice, the concept of ideal thermodynamic adhesion is defined by the work of adhesion $\left(W_{a d h}\right)$, directly linked to the surface free energies of the individual components $\left(\sigma_{1}\right.$ and $\left.\sigma_{2}\right)$ and the interfacial energy $\left(\sigma_{12}\right)$ by the Dupré equation. ${ }^{32}$

$$
W_{a d h}=\sigma_{1}+\sigma_{2}-\sigma_{12}
$$

The work of adhesion represents the energy required per unit area, to separate an interface into two free surfaces, in a realistic cleavage experiment. In Section 7.3.3 we show how the work of adhesion can be computed through nanoindentation simulations. Nonetheless, here we present the work of separation, which serves to approximate the adhesion strength for a given interface. Using the adhesion energy plot (Figure 3.5), we can determine the equilibrium interfacial separation $d_{0}$, by the position at which the minimum energy occurs, and the width at half-maximum of the potential well, the interaction range $l$, which provides an indication of the range over which the two surfaces interact.

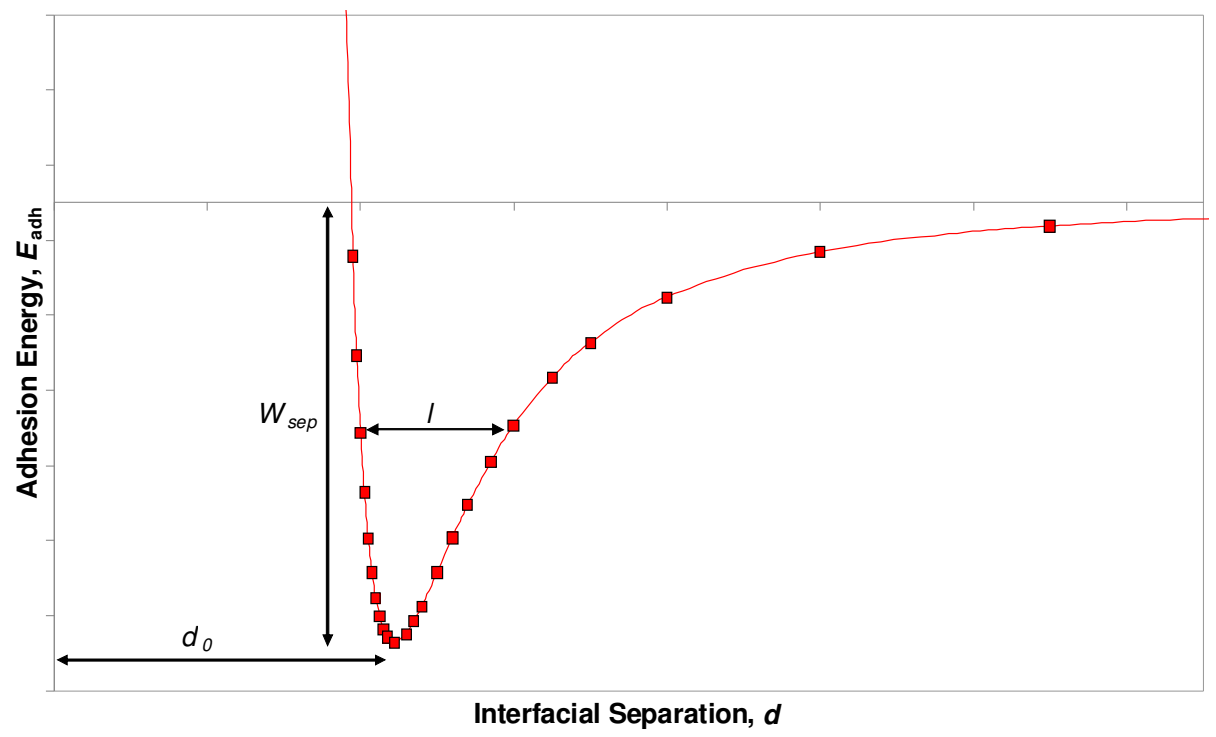

Figure 3.5 Schematic plot of the adhesion energy curve with the work of separation $\left(W_{\text {sep }}\right)$, equilibrium interfacial separation $\left(d_{0}\right)$ and interaction range (I). The work of separation was evaluated by the depth of the potential well of the curve and is a measure of the strength of adhesion at the interface. The equilibrium separation $d_{0}$, is a measure of the typical spacing between surfaces, while the interaction range represents the range over which the two surfaces interact. 
Another measure that can be used to characterize the interaction of the individual surfaces upon formation of the interface is the contact volume $\left(V_{c}\right)$, defined as

$$
V_{c}=V(\infty)-V\left(d_{0}\right)
$$

where $V(\infty)$ is the sum of the occupied volumes of the individual surfaces at infinite separation, and $V\left(d_{0}\right)$ is the occupied volume of the interface at equilibrium interfacial separation. The occupied volumes are calculated as the volume encapsulated by the solvent accessible surface, obtained with a probe of $1.4 \AA$ radius, using the Connolly procedure. ${ }^{144}$ The contact volume $\left(V_{c}\right)$ therefore reflects the extent to which the surfaces come into direct contact at the interface (Figure 3.6). It is important to note that the absolute value of the contact volume depends on the size of the probe and is therefore arbitrary.

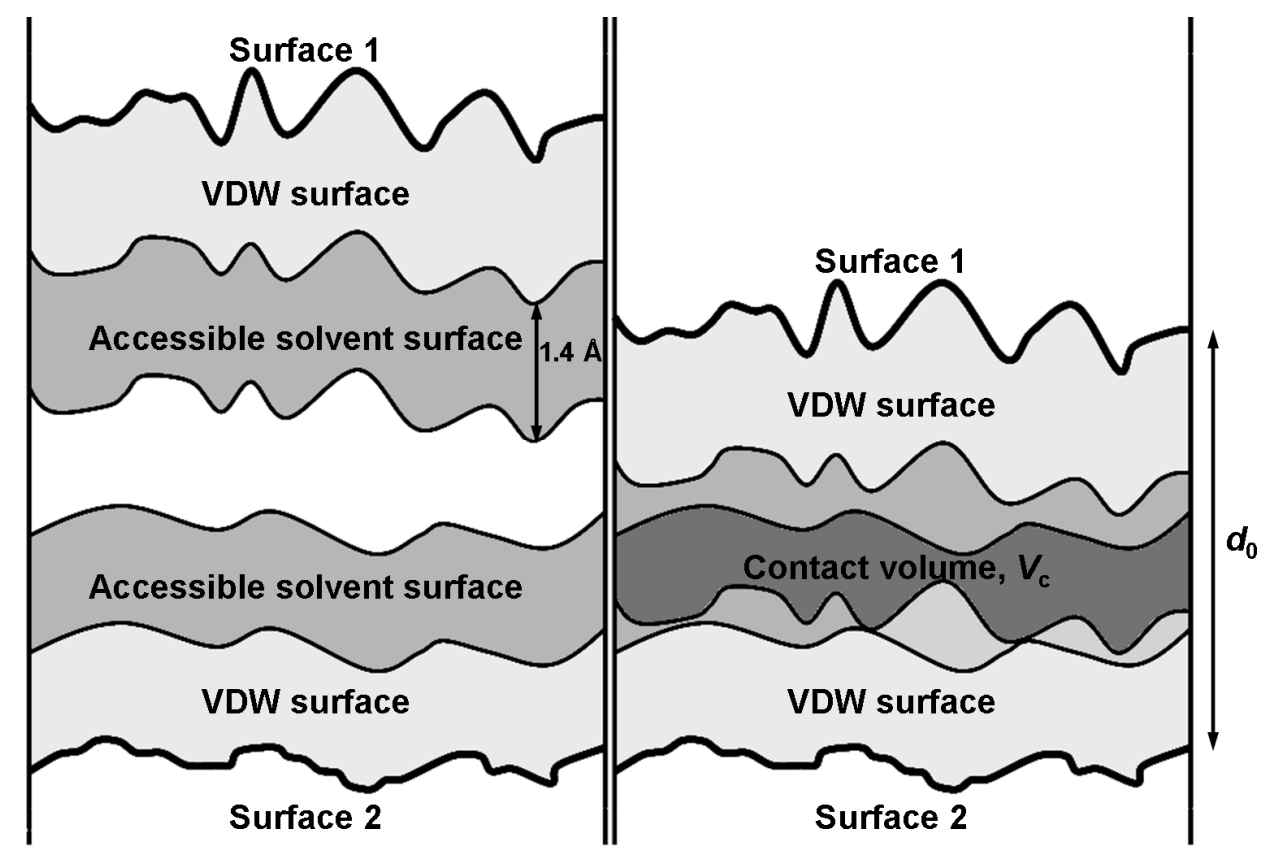

Figure 3.6 Conceptual illustration of the Contact Volume, $V_{c}$. It provides a measure of the effective contact between interacting surfaces.

\subsection{Computational Details}

All calculations were performed using the COMPASS force-field ${ }^{73}$ with the force-field parameters assigned automatically. However, the computational procedures and settings varied slightly depending on the nature of systems, the consideration of surface relaxation and the inclusion of solvent. For this reason, additional details of settings and 
procedures are included in subsequent chapters, pertinent to the particular system in question. Nonetheless, for all single-point energy calculations, the Ewald method was selected to evaluate non-bonded interactions with a convergence limit of $4.0 \times 10^{-4} \mathrm{~kJ}$ $\mathrm{mol}^{-1}$. For the generation of solvent accessible surfaces, the Connolly procedure ${ }^{144}$ was employed, with a grid interval of $0.35 \AA$ and a solvent probe of radius $1.4 \AA$.

Although all surfaces were created to be approximately equal in thickness (18 $)$ ), the boundary of the interface was difficult to identify due to the atomic roughness in the surface profiles. The amorphous nature of the silica, amorphous carbon, and polyester surfaces presented an ambiguity in assigning the exact surface boundary; therefore, for the carbon and unmodified polyester models, it was arbitrarily calculated as the $x-y$ plane positioned at the average $z$ coordinates of all atoms in the leading $2.5 \AA$ of the first bulk atom. For the silica and modified polyesters, where the surface substituents increase the surface roughness, the boundary was determined by the $x-y$ plane positioned at the average $z$ for all atoms comprising the surface modifications plus the atoms in the leading $2.5 \AA$ of the bulk (Figure 3.4). In our initial study, ${ }^{116}$ the leading bulk atom was taken to be the first non-substituent atom and was generally a hydrogen atom. We then modified this slightly to define the leading edge of the bulk to commence at the first non-substituent heavy atom $(\mathrm{O}$ or $\mathrm{C}$ ). This gives a greater consistency across all systems and substituent densities when the interface is composed of two atomically rough surfaces. However, as a result, the $d_{0}$ values reported here are inherently longer by $\sim 0.5$ $\AA$ as compared to those obtained using the initial definition of the polyester surface boundary. ${ }^{116}$ It should be noted, however, that although $d_{0}$ is directly dependent on the definition used for the surface boundaries, the calculated $W_{\text {sep }}$ and interfacial contact volume $\left(V_{c}\right)$ are not. 


\section{Chapter 4}

\section{Effects of Surface Composition and Atomic Roughness on Adhesion}

\subsection{Overview}

In Chapter 3, we described in detail, the polyester and carbon models selected to represent our coating and contaminant materials respectively. In this chapter, we begin our theoretical investigation on the interactions between polyester and amorphous carbon, our coal derived contaminant model. We will take an in-depth look at the effects of atomic-scale roughness and chemical surface composition on adhesion between polyester and amorphous carbon. We will test our various surface modifications of polyester, based on a hydrophilic or alternatively a hydrophobic surface treatment. We will show that these modifications not only impart chemical changes to the surface of the polymer but accentuate its atomic-scale roughness. The initial effects of surface reorganisation will also be investigated by employing energy minimisation techniques that approximate relaxation (see Section 2.4). This surface re-organisation will be shown to be strongly dependent on the structure of the carbon plane, the chemical type, and surface density of modifiers. We will demonstrate that the surface modifiers reorganise during minimisation, which leads to significant differences in their effects on adhesion. 


\subsection{Introduction}

Fabrication of hydrophilic coatings is of great importance for use as potential stay-clean coatings. These coatings remain clean due to principles similar to those used to design contaminant-resistant titanium dioxide windows (see Section 1.3). The main principle is to introduce sites on the surface that favour water uptake and promote the formation of a thin film of water on top of the coating. This film of water prevents contaminants from embedding into the surface thus keeping the coating clean.

A number of experimental studies have investigated hydrophilic modifications of the surface of polyester materials including poly(ethylene terephthalate) (PET) by chemical treatment. ${ }^{145-148}$ For example, Allman et al. ${ }^{146}$ reported a hydrophilic antisoiling agent used for polyester fibers. This protective finish is comprised of polyglycol moieties containing hydroxyl groups available for hydrogen bonding with water. Sufficient hydrophilicity is imparted to the polyester fabric so that aqueous detergent systems can readily wash contaminants away. Similarly, Reeves et al. ${ }^{145}$ reported a carboxymethyl cellulose finish that also imparts hydrophilicity and provides soil resistance to polyester based fabrics. A more recent study includes the work of Montazer et al., ${ }^{148}$ where polyester fabrics were treated in alkaline solution of sodium hydroxide $(\mathrm{NaOH})$ at temperatures greater than $60^{\circ} \mathrm{C}$. Results from this study showed that alkali treatment of polyester caused a significant decrease in the time taken for complete water adsorption due to the incorporation of hydrophilic groups such $-\mathrm{OH}$ and $-\mathrm{COOH}$. Alternative approaches for hydrophilic treatment of polyester surfaces include, oxidation by UV/ozone ${ }^{132,134,149,150}$ and plasma. ${ }^{149,151-160}$. In several of these studies, the increase in hydrophilicity of the polyester is attributed to creation of polar groups at the surface. These groups are formed as a result of oxygen species which attack the C-C and C-H pendant groups of the surface rather than the ester groups in the polymer chain. These surface treatment lead not only to the formation and/or introduction of polar hydrophilic groups, but can also lead to surface crosslinking, ${ }^{134,150,157-160}$ and even changes in surface morphology. ${ }^{154,160}$

An alternative class of self-cleaning surface is super-hydrophobic coatings. The benchmark model for a superhydrophobic self-cleaning surface is provided by the leaves 
of the Lotus plant. The surface of the leaf is characterised by a high contact angle and low roll-off angle (low contact angle hysteresis) which means that water droplets can easily roll off the leaf carrying with them undesirable particulates (see Section 1.3 for a complete description on the stay-clean mechanism of the lotus leaf).

One of the most widely used methods for improving hydrophobicity of selected polymeric surfaces is through surface fluorination. For polymer films with initially high surface energy, the incorporation of fluorine groups to the surface generally reduces the surface energy of the film. It is this reduction in surface energy which results in an increase in water contact angle, and a reduction in the interaction between water and surface, rendering the polymer hydrophobic (water repellent). Indeed many experimental studies have shown that fluorine modifications of polymer based surfaces can increase water repellence. ${ }^{36,38-42}$ In one example, Selli et al. ${ }^{39}$ investigated the possibility of imparting water repellence and soil-resistance properties to various fabrics including PET fibers through surface fluorination using cold sulfur hexafluoride $\left(\mathrm{SF}_{6}\right)$ plasma treatment. Plasma treatment led to the implantation of fluorine atoms on the surface of the polymer, and this resulted in increased water repellence as indicated by the increase in water absorption time after treatment. In another investigation, plasma modification of a PET fibre surface was shown to increase the water contact angle from $79^{\circ}$ to $101^{\circ}$ indicating an increase in hydrophobicity. ${ }^{42}$

The control of chemical groups on a surface is an important aspect for the successful application of stay-clean coatings. Another important factor that needs to be considered here is the surface morphology and specifically the fine-scale surface roughness of the coating. Surface roughness can have an appreciable impact on interfacial adhesion, depending on the size and spacing of the surface protrusions. Zhou at al. ${ }^{161}$ investigated the effects of nano-scale surface roughness on the adhesion of spherical polymer particles on various substrates by means of AFM and centrifugal detachment experiments. Their results showed, the vdW adhesion force can be significantly reduced by minimising the density between the two adhering materials. This was achieved by introducing an appropriate surface roughness based on RMS (root-mean-squared) value and peak-to-peak distance. Similarly, Rabinovich et al. ${ }^{162,163}$ found that the adhesion between surfaces or between a particle and a surface can be reduced by minimising the 
real area in contact and increasing the distance between the bulk surfaces. However, Israelachvilli ${ }^{164}$ has shown that while surface roughness can reduce adhesion between stiff copolymer films, for more flexible rough surfaces the opposite behaviour can occur, whereby interdigitation and chain entanglement can result in higher interfacial adhesion energy. This observation is again believed to be related to the real contact area in question.

In this section, we construct simple models of surface oxidised polyesters by introducing hydroxyl $(\mathrm{OH})$ or carboxyl $\left(\mathrm{CO}_{2} \mathrm{H}\right)$ functional groups to the polymer surface, while fluorine atoms are introduced to model fluorinated polyester films. Subsequently, each polymer layer is placed against an amorphous carbon model (a realistic representation of industrial char) and the resultant adhesion strength between the carbon and polymer in vacuum is calculated by means of adhesion modelling (technique described in Section 3.4). It has been suggested ${ }^{2,95}$ that due to the hydrophilic nature of silica surfaces ${ }^{165}$ they may be less susceptible to adhesion of carbonaceous materials than organic polymers such as polyester. In fact polyester is often coated with thin silica films $s^{2,165}$ to render the material hydrophilic, and impart soil resistant properties to the surface. Therefore, to investigate the mechanism of the soil resistance and establish a benchmark, we also examined the interaction of a model surface of silica with carbon. Finally, we employ energy minimisation (see Section 2.4) to determine the initial effects of relaxation, on the surface structure and chemical composition of the modified polyester films.

\subsection{Molecular Models and Theoretical Procedures}

\subsubsection{Composition of Molecular Models}

The unmodified polyester surface (Polyester) and modified polyesters (Polyster $n \mathrm{X}$ ) were prepared as described in Section 3.2. In the case of silica, we used a realistic model of a vitreous silica surface, based on the work of Garofalini and co-workers ${ }^{166-169}$ which has been modified as reported previously ${ }^{170,171}$ to produce a hydroxylated amorphous silica surface. The silica model was prepared with surface hydroxylation of $2.2 \mathrm{OH}$ groups per $\mathrm{nm}^{2}$ (35 hydroxyl groups per unit cell area, Silica35), which represents a partially hydrated silica surface. Zhuravlev ${ }^{172}$ reported that a completely hydrated silica surface 
had a silanol density of $\sim 5.0 \mathrm{OH}$ groups per $\mathrm{nm}^{2}$. For all subsequent simulations, the surface silanol groups remained free to move, while the underlying silica was held in a fixed geometry.

The carbon contaminant was modelled using a hydrogenated amorphous carbon structure $(\mathrm{AmCH})$ comprised of six disordered and buckled graphite-like sheets. It is based on a previously constructed 3-D periodic model of an amorphous industrial char. ${ }^{139}$ A detailed description of the model can be found in Section 3.3.

All surface models were created to be approximately the same size with an area of $40 \AA \mathrm{x} 40 \AA$ ( $x$ and $y$ directions) and a thickness of approximately $18 \AA$ ( $z$ direction). A total of 12 interface systems were formed by combining amorphous carbon with all 11 polyester surfaces and silica. The systems are denoted Systems $N \mathrm{~b}$, where " $N$ " represents the polymer surface from Table 3.1, and "b" represents the carbon model from Section 3.3 (in this case, amorphous carbon). A schematic representation of a typical interface has previously been displayed in Figure 3.4. Initially, a vacuum spacer was inserted between the surfaces to represent the interfacial separation of the system. The simulation box was further extended to $300 \AA$ in the $z$ direction to ensure that adjacent systems do not interact along the $z$ axis when 3-D periodic boundary conditions are imposed. As a result, quasi 2-D periodic interfaces were created, where infinite surfaces in contact were mimicked.

\subsubsection{Theoretical Procedures}

Following formation of each system, the adhesion energy was monitored as a function of interfacial separation, as described in detail in Section 3.4. Interfacial properties such as work of separation $\left(W_{\text {sep }}\right)$, and equilibrium separation $\left(d_{0}\right)$, were evaluated helping characterize the strength of interaction between polymer and carbon.

The effect of surface roughness on interfacial adhesion was investigated by employing two different computational procedures. In the first procedure, (Procedure A) the systems were held rigid and stiff (Systems $N b^{\text {rig }}$ ). The adhesion energy of these systems was then monitored as the idealistic rigid polyester models were brought from infinite separation toward the amorphous carbon surface to form the interface. 
Suppressing relaxation during interfacial formation gives an approximate value for the work of separation $\left(W_{\text {sep }}^{a p p}\right)$.

In the second procedure, (Procedure B) local relaxation of the interfaces was undertaken by taking the rigid structure at the equilibrium separation from Procedure A, and then energy minimising the polyesters over the fixed carbon surface. This process leads the energy of the interface structure to its nearest local energy minimum (see Section 2.4). The resulting systems are referred to as minimised interfaces and denoted $\mathrm{Nb}^{\mathrm{min}}$. Adhesion energy curves were also obtained from these minimised interfaces by determining the adhesion energy at different interfacial separations $\left(E_{a d h}(d)\right)$ as the polyesters were withdrawn from the interface without further relaxation.

We also attempted to isolate chemical and structural contributions to the adhesion energy of the interface. ${ }^{173}$ The adhesion energy can be considered as the sum of structural contributions $\left(E_{\text {struc }}\right)$ and chemical contributions $\left(E_{\text {chem }}\right)$

$$
E_{\text {adh }}(d)=E_{\text {struc }}(d)+E_{\text {chem }}(d)
$$

The energy components were acquired by (a) isolating the modified polyester models (PolyesternX) for each interface in its energy minimised configuration, (b) replacing the $\mathrm{F}, \mathrm{CO}_{2} \mathrm{H}$, or $\mathrm{OH}$ substituents with hydrogen atoms, with redetermination of the partial atomic charges to maintain neutrality, and (c) revaluating the work of separation for these hydrogenated interfaces $\left(W_{\text {sep }}{ }^{H}\right)$. This is equivalent to obtaining the $W_{\text {sep }}$ of the unmodified interface (System 1b) "locked" in the structural configuration of each of the energy minimised Polyester $n \mathrm{X}$ interfaces. The structural component $\left(\Delta W_{\text {sep }}^{\text {struc }}\right)$ is then the difference in $W_{\text {sep }}$ values of the original unsubstituted (unmodified) interface and that of hydrogenated systems, i.e.

$$
\Delta W_{\text {sep }}^{\text {struc }}=W_{\text {sep }}^{H}-W_{\text {sep }}^{\text {Sys.1 } b^{\min }}
$$

The parameter $\Delta W_{\text {sep }}^{\text {struc }}$ represents the difference in $W_{\text {sep }}$ values attributable to structural variations in the polyester structure. The chemical component $\left(\Delta W_{\text {sep }}^{\text {chem }}\right)$ is defined as the difference in work of separation values between the hydrogenated system and the chemically modified interface, i.e. 


$$
\Delta W_{\text {sep }}^{\text {chem }}=W_{\text {sep }}-W_{\text {sep }}{ }^{H}
$$

Consequently, the overall change in the work of separation resulting from modification $\left(\Delta W_{\text {sep }}\right)$ can now be considered as a sum of structural $\left(\Delta W_{\text {sep }}^{\text {struc }}\right)$ and chemical changes $\left(\Delta W_{\text {sep }}^{\text {chem }}\right)$.

\subsection{Results and Discussion}

\subsubsection{Rigid Interfaces (Systems $\mathrm{Nb}^{\text {rig }}$ )}

Table 4.1 presents key interaction parameters for all rigid interfaces (Systems $N b^{\text {rig }}$ ), including approximate work of separation $\left(W_{\text {sep }}{ }^{a p p}\right)$, equilibrium interfacial separation $\left(d_{0}\right)$, interaction range $(l)$, atomic density of the polymer $\left(\rho_{2}\right)$, and contact volume $\left(V_{c}\right)$.

Table 4.1 Adhesion Parameters for Rigid Interfaces (Systems $\mathrm{Nb}^{\text {rig }}$ )

\begin{tabular}{rlccccc}
\hline System & $\begin{array}{c}W_{\text {sep }}^{\text {app }} \\
\left(\mathrm{mJ} \mathrm{m}^{-2}\right)\end{array}$ & $\begin{array}{c}d_{0} \\
(\AA)\end{array}$ & $\begin{array}{c}l \\
(\AA)\end{array}$ & $\begin{array}{c}\rho_{2} \\
\left(\mathrm{~atm} \mathrm{~nm} \mathrm{~m}^{-3}\right)\end{array}$ & $\begin{array}{c}V_{c} \\
\left(\AA^{3}\right)\end{array}$ \\
\hline $1 \mathrm{~b}^{\text {rig }}$ & polyester / AmCH & 116.0 & 4.22 & 2.00 & 88.2 & 1842 \\
$2 \mathrm{~b}^{\text {rig }}$ & Polyester7F/AmCH & 109.9 & 4.36 & 2.04 & 86.4 & 1766 \\
$3 \mathrm{~b}^{\text {rig }}$ & Polyester7OH/AmCH & 102.7 & 4.46 & 2.07 & 80.5 & 1634 \\
$4 \mathrm{~b}^{\text {rig }}$ & Polyester7CO $2 \mathrm{H} / \mathrm{AmCH}$ & 106.0 & 4.47 & 2.06 & 80.9 & 1644 \\
$5 \mathrm{~b}^{\text {rig }}$ & Polyester15F/AmCH & 112.4 & 4.35 & 2.03 & 85.8 & 1792 \\
$6 \mathrm{~b}^{\text {rig }}$ & Polyester15OH/AmCH & 105.2 & 4.44 & 2.07 & 81.0 & 1703 \\
$7 \mathrm{~b}^{\text {rig }}$ & Polyester15CO $\mathrm{H} / \mathrm{AmCH}$ & 110.7 & 4.44 & 2.04 & 80.7 & 1722 \\
$8 \mathrm{~b}^{\text {rig }}$ & Polyester(25x3)F/AmCH & 114.7 & 4.47 & 2.03 & 83.3 & 1867 \\
$9 \mathrm{~b}^{\text {rig }}$ & Polyester(25x3)OH/AmCH & 112.8 & 4.54 & 2.06 & 85.2 & 1830 \\
$10 \mathrm{~b}^{\text {rig }}$ & Polyester75F/AmCH & 113.2 & 4.52 & 2.02 & 86.2 & 1916 \\
$11 \mathrm{~b}^{\text {rig }}$ & Polyester75OH/AmCH & 117.1 & 4.45 & 2.04 & 86.5 & 1947 \\
$12 \mathrm{~b}^{\text {rig }}$ & Silica35/AmCH & 86.9 & 4.82 & 2.15 & 57.6 & 1127
\end{tabular}




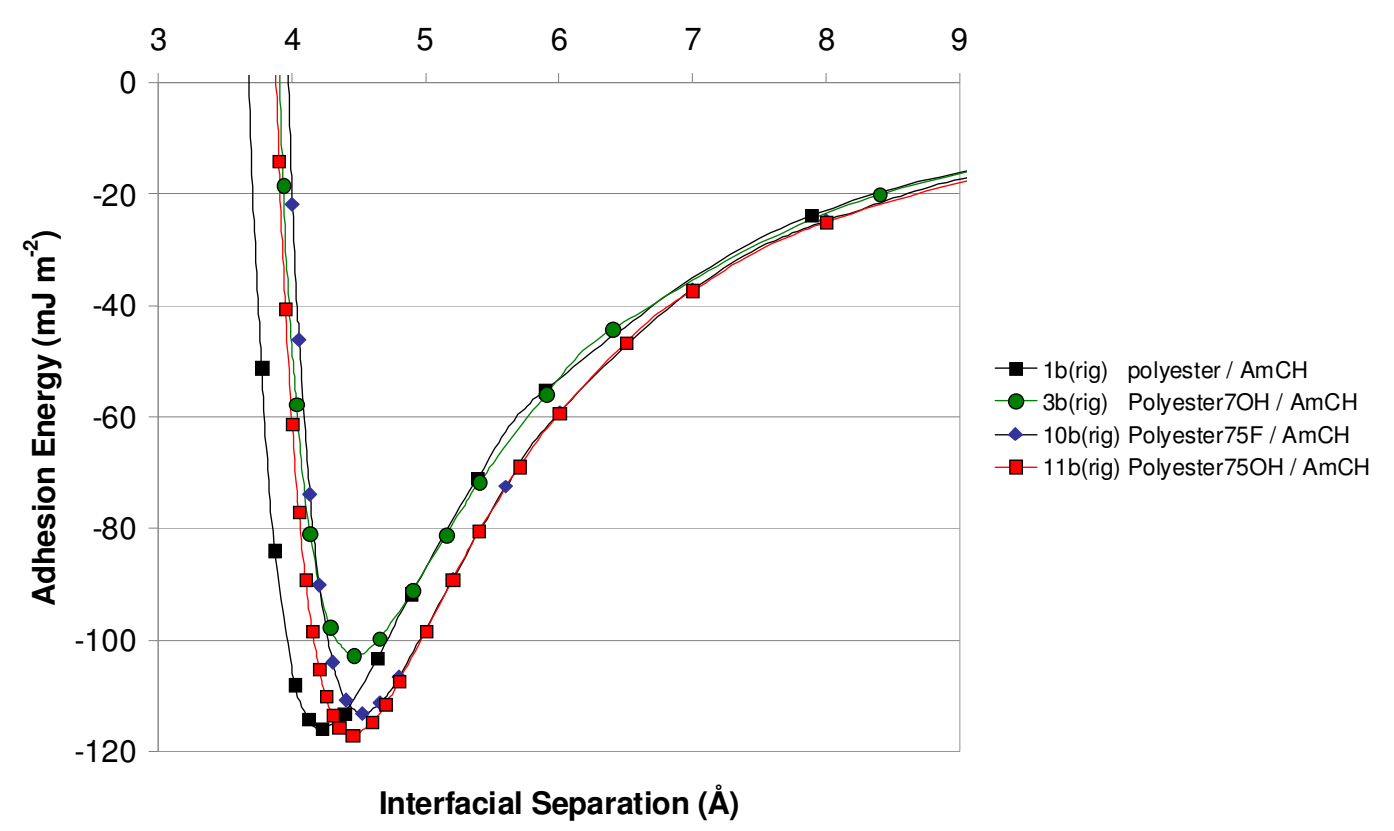

Figure 4.1 Adhesion energy plots of modified polyesters interacting with amorphous carbon $(\mathrm{AmCH})$. The unmodified interface, polyester/ $\mathrm{AmCH}$, is also included as a reference point. The depth of the well in these curves provides a measure of the adhesion at the interface, and the greater the dip, the stronger the adhesion between coating (polymer) and contaminant $(\mathrm{AmCH})$. Adhesion plots show that low-level hydroxyl (7OH) surface modifications reduce adhesion between coating and contaminant.

Figure 4.1 shows representative interfacial adhesion curves of selected rigid systems. According to the approximate work of separation parameter $\left(W_{\text {sep }}{ }^{a p p}\right)$ of Table 4.1, Silica35/AmCH interface (System $12 \mathrm{~b}^{\mathrm{rig}}$ ) exhibits a weaker interfacial adhesion $(\sim 25 \%)$ compared to the polyester/AmCH interface (System $\left.1 b^{\text {rig }}\right)$. It can also be seen from Table 4.1 that the equilibrium interfacial separation $d_{0}$, between silica and amorphous carbon model is greater than the polyester/AmCH system. The silica surface used in this present study is atomically rough, reducing the effective contact at the interface and decreasing the $\mathrm{vdW}$ interaction between the interacting surfaces. Supporting this is the observation that the contact volume $V_{c}$, of the Silica35/AmCH interface is $\sim 39 \%$ less than for the polyester/AmCH interface. The weaker adhesive interaction between silica and carbon as compared to polyester and carbon is consistent with experiment ${ }^{2,174}$ and provides a qualitative measure of the validity of the COMPASS force-field used to characterize these interfacial interactions. It is also in agreement with 
our previous study that showed that silica exhibits a weaker interfacial adhesion with graphite than polyester. ${ }^{116}$

Under this rigid regime (Procedure A), where the polyester model is artificially kept to an atomically rough surface profile, surface modification is generally shown to reduce interfacial interaction between polyester and amorphous carbon. Observation of Table 4.1 reveals that the majority of modified systems exhibit reductions in interfacial adhesion $\left(W_{\text {sep }}^{a p p}\right)$ and contact volume $\left(V_{c}\right)$ relative to the base interface (polyester/AmCH). In conjunction with this, the modified interfaces exhibit a larger equilibrium interfacial separation $\left(d_{0}\right)$ and interaction range $(l)$ than the polyester/AmCH system.

It can be seen from Table 4.1 that the magnitude of these adhesive reductions may vary depending on the chemical type and level of modification undertaken. Low-level functionalisation (Polyester7X interfaces, 0.4 residues per $\mathrm{nm}^{2}$ ) is shown to lead to a $\sim 5$ $11 \%$ reduction in interfacial adhesion, relative to the base interface. For models with surface functionalisation of 0.9 residues per $\mathrm{nm}^{2}$ (Polyester $15 \mathrm{X}$ interfaces), the reduction in the work of separation is slightly smaller $(\sim 3-9 \%)$. High levels of functionalisation (Polyester(25 x 3)X and Polyester75X systems) exhibit a negligible reduction in adhesion ( 1-3\%), and in the case of the Polyester75OH/AmCH system, a slight increase in $W_{\text {sep }}{ }^{a p p}$ is predicted. Overall, low-level hydroxylation of the polyester surface leads to the largest reduction in adhesion to amorphous carbon $(\sim 11 \%)$. These observations suggest that only a small number of surface modifiers are required to significantly reduce adhesion $\left(W_{\text {sep }}{ }^{a p p}\right)$ to amorphous carbon under this rigid regime, and increasing the level of modification for the rigid polyester surface does not lead to further reductions in adhesion. This was also observed in the case of the modified polyester and graphite interfaces. ${ }^{116}$

Clearly, there are a number of competing factors that contribute to the overall interaction between the surfaces. The amorphous carbon model used in this study differs from the previously used graphite, in that its constituent carbon atoms carry partial atomic charges. However, analysis of the non-bonded contributions indicate that these variations in $W_{\text {sep }}{ }^{a p p}$ are attributed primarily to changes in the vdW interaction energy at the interface, as electrostatic interactions contribute a negligible amount to the overall 
work of separation $(<1.2 \%)$. The low contribution of the coulomb forces to the interaction between polymers and graphite-like structures has also been identified through the theoretical work of Yang et al. ${ }^{115}$ They found that it was vdW interactions that drive physisorption of conjugated polymers, like polyester, onto carbon nanotubes.

The vdW interaction energy between two macroscopic infinite surfaces can be characterized using the Hamaker relation ${ }^{13}$

$$
W\left(d_{0}\right) \propto-\pi^{2} C_{12} \rho_{1} \rho_{2}
$$

where $W\left(d_{0}\right)$ is the vdW interaction energy at interfacial separation $d_{0}, C_{12}$ is the coefficient of atom-atom pair potential and reflects the chemical composition of the surfaces, and $\rho_{1}$ and $\rho_{2}$ are the atomic densities of surfaces 1 and 2, respectively. A reduction in $\rho_{2}$ can lessen the overall $\mathrm{vdW}$ interfacial interaction energy, provided that the remaining properties are kept constant. Inspection of Table 4.1 reveals that the Polyester7X and Polyester15X modified surfaces exhibit a $\sim 2-9 \%$ reduction in atomic density $\left(\rho_{2}\right)$ relative to the base polyester. In contrast, functionalised polyesters with higher concentrations of surface substituents (Polyester75X or Polyester(25 x 3)X surfaces) exhibit a $\sim 3-6 \%$ reduction in atomic density relative to the untreated model. These trends generally accompany the observed variations in $W_{\text {sep }}{ }^{a p p}$; that is, modified polymers with relatively low atomic density, display a reduced strength of adhesion with carbon. Reductions in atomic density arise from the greater protrusion of the functional groups $\left(\mathrm{OH}, \mathrm{CO}_{2} \mathrm{H}\right.$, and $\left.\mathrm{F}\right)$ as compared with the hydrogen atoms that they replace. In simple terms, the presence of surface substituents accentuates the fine-scale roughness of the polyester model.

However, the atomic density alone is not sufficient to accurately describe all trends in adhesion $\left(W_{\text {sep }}{ }^{a p p}\right)$. For example, the Polyester7F surface exhibits a larger atomic density than the Polyester15F model, yet leads to weaker adhesion with amorphous carbon. Also, the Polyester75OH/AmCH interface exhibits a stronger work of separation value than the base system despite the fact that Polyester75OH exhibits a lower atomic density than the base polyester. Therefore, we must look at additional factors that contribute to these trends. 


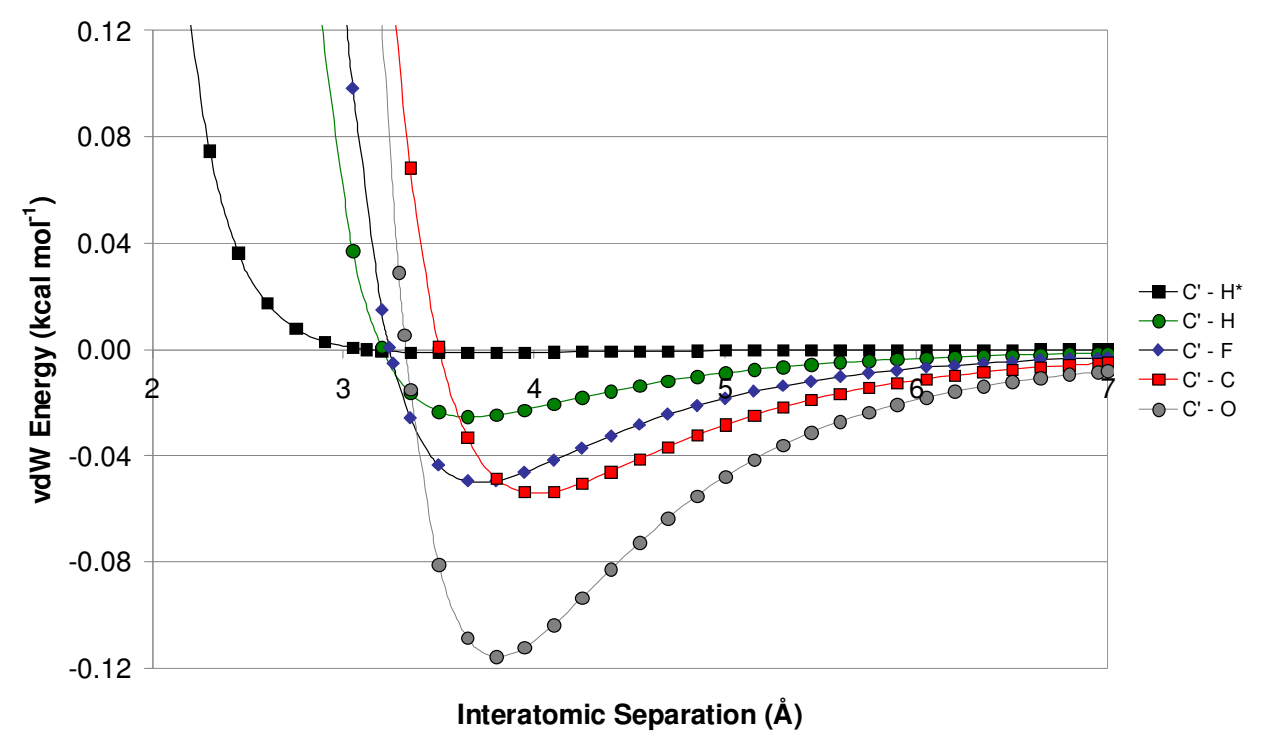

Figure 4.2 Plots of the vdW energy between atoms as a function of their interatomic separation. These plots compare the vdW interactions between polymer surface atoms (hydrogen, fluorine, carbon and oxygen) with aromatic carbons of graphite (C'). 'Heavy' atoms such as F, C and $\mathbf{O}$ offer a relatively strong repulsion with aromatic carbon at short interatomic separations, while hydrogen $\left(\mathrm{H}\right.$ or $\left.\mathrm{H}^{\star}\right)$ offers a relatively weak repulsion.

Modifications were undertaken by replacement of terminal hydrogens $(H)$ that protrude on the surface of the base polyester, with fluorine $(\mathrm{F})$, carboxyl $\left(\mathrm{CO}_{2} \mathrm{H}\right)$, and hydroxyl $(\mathrm{OH})$ residues. Therefore, to account for variations in interfacial adhesion, it is important to look at the strength of $\mathrm{vdW}$ interactions between these surface atoms and the aromatic carbons $\left(C^{\prime}\right)$ that constitute the majority of atoms along the surface of the amorphous carbon model. Interatomic potential curves of the C'-O, C'-C, C'-F, and C'$\mathrm{H}$ atom pairs are shown in Figure 4.2. It is clear from these plots that all polyester surface atoms exhibit a short-range repulsion and long-range attraction with aromatic carbon. Carbon exhibits the strongest short-range repulsion, followed by oxygen, fluorine, and hydrogen. On the other hand, oxygen exhibits the strongest long-range attraction. Of the atom types examined here, $\mathrm{H}^{*}$ (hydrogen located in hydrophilic functional groups, $\mathrm{OH}$, and $\mathrm{CO}_{2} \mathrm{H}$ ) has the weakest repulsive and dispersive component with aromatic carbon. However, the most important feature of Figure 4.2 is that the hydrogen $(\mathrm{H})$, which makes up a large percentage of the base polyester surface atoms, exhibits a weaker repulsive and dispersive component than fluorine, oxygen, and carbon. 
Replacement of these $\mathrm{H}$ atoms with functional groups containing heavier substituents should therefore lead to a stronger interaction between polyester and amorphous carbon i.e., stronger repulsion at short interfacial separations and stronger attraction at large interfacial separations. Adhesion energy plots presented in Figure 4.1 reveal that at large interfacial separations ( $>5 \AA$ ), modified systems indeed exhibit a stronger attraction to amorphous carbon compared to the unmodified system. As a consequence of this, functionalised interface systems display slightly larger peak width values $(l)$ than the base interface.

Clearly the presence of surface modifiers perturbs the vdW interaction at the interface. Such chemical modifications can lower adhesion between polymer and carbon depending on the position of the modifiers relative to the carbon model. Modification was carried out, by replacement of the most accessible sites on the surface first. In addition to this, the polyester models were held rigid. The consequence of these two conditions is that at equilibrium separation $\left(d_{0}\right)$, the majority of surface modifiers for low-level functionalised polymers (Polyester7X) are located in close proximity and within $\sim 3.5 \AA$ from the nearest carbon atom of the contaminant model. From this distance, the heavy substituents offer a stronger repulsion to carbon compared to the light hydrogen atoms that they replace, reducing the overall strength of adhesion. This chemical effect combined with the accentuated roughness of protruding substituents noted earlier, leads to relatively large reductions in adhesion for low-level functionalised systems. Hence, the Polyester7X systems display the largest reductions in $W_{\text {sep }}{ }^{a p p}$ and $V_{c}$ and the largest increase in $d_{0}$ relative to the unmodified interface. However, the strength of the repulsive interaction is reduced as the functional groups are positioned deeper into the polymer and therefore further from the carbon surface. If the group is placed in one of the polymer surface cavities, a flattening of the polymer surface occurs, and if the functional group is located far enough from the carbon particle, the attractive contribution may increase. The additional residues present in the Polyester15X models as compared to the Polyester7X surfaces are indeed positioned progressively deeper toward the bulk of the polyester. As a result, the Polyester15X models exhibit a stronger interfacial adhesion (increased work of separation values) than the Polyester7X models. However, it appears that the initial $7 \mathrm{X}$ groups of the Polyester15X model exhibit a 
strong repulsion with amorphous carbon, which is sufficient to hold the surfaces apart to the same extent as the Polyester7X models. Therefore, there appears to be little change in the $d_{0}$ values of the Polyester15X/AmCH interfaces relative to the Polyester7X/AmCH systems). This is an extremely important point, as it reflects the complex balance between the repulsion of the high substituents and the attraction of the deeper substituents. For the Polyester75X and Polyester(25 x 3)X interfaces, this complex saturation effect is further enhanced, as these models exhibit a stronger adhesion to amorphous carbon as compared to their Poylester15X counterparts, and in all these cases, there is not only an increase in $W_{\text {sep }}$ but also a slightly larger equilibrium separation $d_{0}$. Overall, the results presented here lead to the same qualitative trends as observed in the case of graphite. ${ }^{116}$ In particular, the combination of chemical and physical effects (atomic-scale surface roughness) from the introduction of modifiers has been shown to significantly reduce adhesion to carbon surfaces (graphite or amorphous carbon) by reducing the overall $\mathrm{vdW}$ interaction at the interface. For atomically rough polymer surface, we only require a small number of surface substituents for optimal adhesive resistance. The carbon surface acts as a hard wall and when combined with the rigid atomically rough modified polyester surface experiences a strong repulsion at close interfacial separation, similar to the observation made by Israelachvili and co-workers. ${ }^{164}$

One note of difference between the polymer/amorphous carbon systems and their graphite equivalents, ${ }^{116}$ is that surface modifications appear to be more effective in reducing interfacial adhesion with graphite than with amorphous carbon. For example, the introduction of $\mathrm{F}, \mathrm{CO}_{2} \mathrm{H}$, or $\mathrm{OH}$ functional groups at a surface coverage of 0.4 residues per $\mathrm{nm}^{2}$ (Polyester7X models), results in a $\sim 5-11 \%$ reduction in adhesion between polyester and amorphous carbon. In the case of graphite, these modifications lead to a $\sim 16-22 \%$ adhesive reduction. As graphite is atomically smooth, it provides an equivalent interaction across the entire contact area of the interface. In comparison, the amorphous carbon consists of less ordered, buckled graphitic layers resulting in an irregular contoured surface, which inevitably leads to asymmetric interactions across the interfacial contact area. Interactions of protruding modifiers of the polymer with convex regions of the amorphous carbon will generally lead to similar repulsive interactions as with graphite. However, interactions of the polymer modifiers with concave regions of 
the amorphous carbon will generally lead to reduced repulsive interactions and/or attractive interactions at the interface. Therefore, the irregular surface profile of the amorphous carbon reduces the effectiveness of surface modification under artificially rigid conditions.

\subsubsection{Energy Minimised Interfaces (Systems $N b^{\text {min }}$ )}

Figure 4.3 displays adhesion curves of selected interfaces from Procedures A and B. Table 4.2 in the following page, presents the key interaction parameters for all interfaces studied using Procedure B. The polyester models were allowed to relax against the rigid amorphous carbon surface, using an energy minimisation technique that leads the structure to the nearest local minimum.

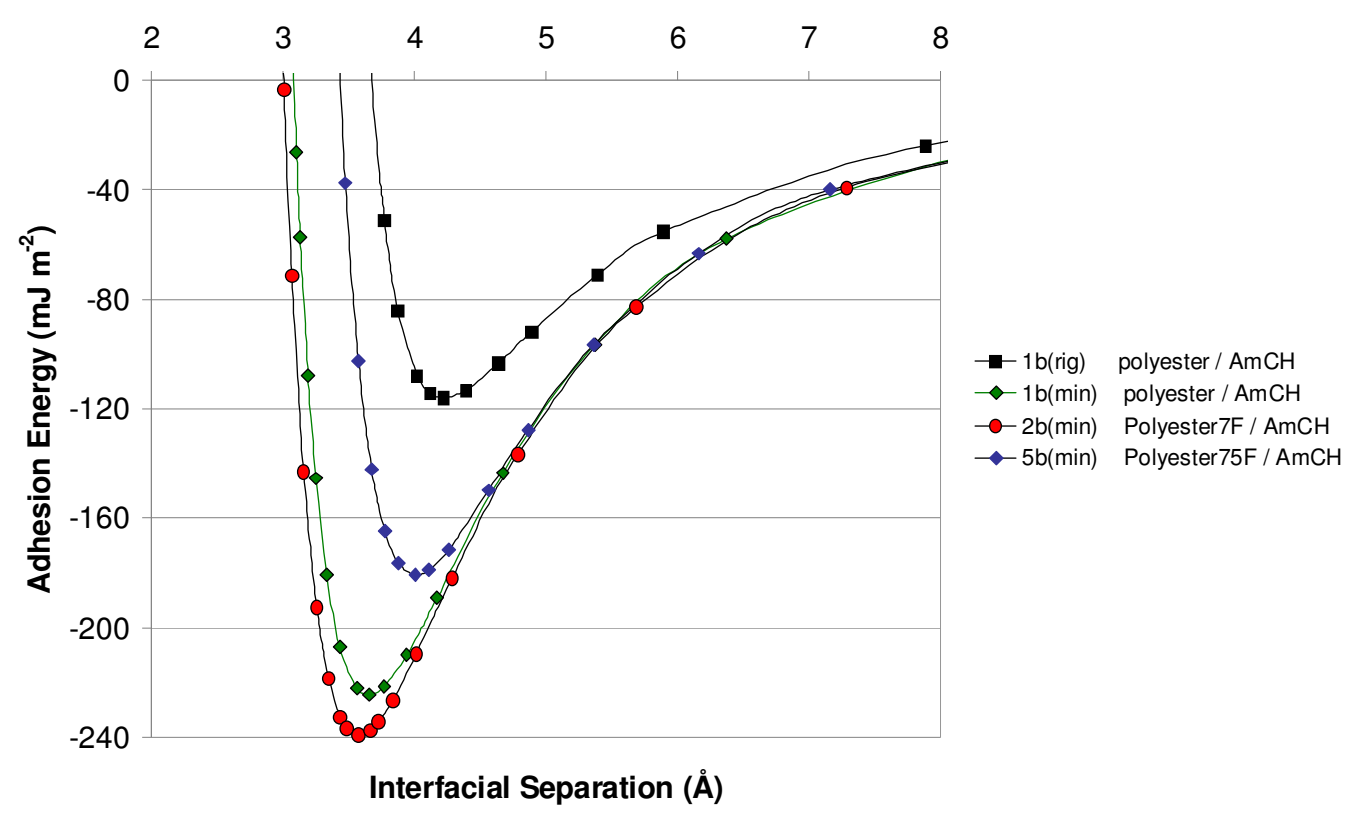

Figure 4.3 Adhesion energy plots for polymer/amorphous carbon interfaces highlighting the importance of rigidity as a preventative measure for surface contamination. The amorphous carbon surface represents a carbon contaminant, and the polyesters represent various paint coatings. The depth of the well in these plots provide a measure of the adhesion at the interface, and the greater the dip, the stronger the adhesion between coating and contaminant. The amorphous carbon contaminant is seen to adhere considerably weaker to the rigid and atomically rough polyester than the energy minimised polymers. 
Table 4.2 Adhesion Parameters for Energy Minimised Interfaces (Systems $\mathrm{Nb}^{\mathrm{min}}$ )

\begin{tabular}{|c|c|c|c|c|c|c|c|c|c|}
\hline & System & $\begin{array}{c}W_{\text {sep }} \\
\left(\mathrm{mJ} \mathrm{m}^{-2}\right)\end{array}$ & $\begin{array}{c}d_{0} \\
(\AA)\end{array}$ & $\begin{array}{l}l \\
(\AA)\end{array}$ & $\begin{array}{c}\rho \\
\left(\operatorname{atm} \mathrm{nm}^{-3}\right)\end{array}$ & $\begin{array}{c}V_{c} \\
\left(\AA^{3}\right)\end{array}$ & $\begin{array}{c}W_{\text {sep }}{ }^{H} \\
\left(\mathrm{~mJ} \mathrm{~m}^{-2}\right)\end{array}$ & $\begin{array}{l}{ }^{\mathrm{iv}} \Delta W_{\text {sep }}^{\text {chem }} \\
\left(\mathrm{mJ} \mathrm{m}^{-2}\right)\end{array}$ & $\begin{array}{c}{ }^{\mathrm{v}} \Delta W_{\text {sep }}{ }^{\text {struct }} \\
\left(\mathrm{mJ} \mathrm{m}^{-2}\right)\end{array}$ \\
\hline $1 b^{\min }$ & polyester/AmCH & 224.5 & 3.65 & 1.90 & 84.6 & 3599 & & & \\
\hline $2 b^{\min }$ & Polyester7F/AmCH & 239.3 & 3.58 & 1.91 & 84.1 & 3704 & 238.4 & 0.9 & 13.9 \\
\hline $3 b^{\min }$ & Polyester7OH/AmCH & 216.5 & 3.73 & 1.90 & 83.7 & 3504 & 213.2 & 3.3 & -11.3 \\
\hline $4 b^{\min }$ & Polyester $7 \mathrm{CO}_{2} \mathrm{H} / \mathrm{AmCH}$ & 239.1 & 3.54 & 1.90 & 79.3 & 3673 & 233.0 & 6.1 & 8.5 \\
\hline $5 b^{\min }$ & Polyester15F/AmCH & 228.1 & 3.60 & 1.91 & 84.2 & 3633 & 225.0 & 3.1 & 0.5 \\
\hline $6 b^{\min }$ & Polyester15OH/AmCH & 217.9 & 3.57 & 1.91 & 81.3 & 3528 & 211.2 & 6.7 & -13.3 \\
\hline $7 b^{\min }$ & Polyester $15 \mathrm{CO}_{2} \mathrm{H} / \mathrm{AmCH}$ & 243.5 & 3.63 & 1.91 & 83.9 & 3708 & 224.4 & 19.1 & -0.1 \\
\hline $8 b^{\min }$ & Polyester(25×3)F/AmCH & 205.9 & 3.84 & 1.92 & 84.1 & 3298 & 196.8 & 9.1 & -27.7 \\
\hline $9 b^{\min }$ & Polyester(25×3)OH/AmCH & 210.0 & 3.77 & 1.93 & 83.7 & 3256 & 187.5 & 22.5 & -37.0 \\
\hline $10 b^{\min }$ & Polyester75F/AmCH & 180.8 & 4.00 & 1.94 & 82.2 & 2888 & 171.8 & 9.0 & -52.7 \\
\hline $11 b^{\min }$ & Polyester75OH/AmCH & 220.0 & 3.78 & 1.94 & 81.7 & 3418 & 194.2 & 25.8 & -30.3 \\
\hline
\end{tabular}

\footnotetext{
iv $\Delta W_{\text {sep }}^{\text {chem }}=W_{\text {sep }}-W_{\text {sep }}{ }^{H}$

${ }^{\mathrm{v}} \Delta W_{\text {sep }}^{\text {struc }}=W_{\text {sep }}{ }^{H}-W_{\text {sep }}^{\text {Sys. } 1 b^{\mathrm{min}}}$
} 
It is clear from Table 4.2 and Figure 4.3 that energy minimisation leads to a significant increase in interfacial adhesion $\left(W_{\text {sep }}\right)(\sim 94 \%)$, for the base system (polyester/AmCH) relative to its equivalent rigid interface. In conjunction with this, there is a corresponding decrease in the equilibrium separation $(\sim 0.6 \AA)$ and a rise in the interfacial contact volume ( $95 \%)$. In comparison, the local relaxation procedure of the polyester/graphite interface led to an increase in adhesion ( $\left.W_{\text {sep }}\right)$ of only $\sim 65 \%$.

These same trends are also observed for the modified systems (PolyesternX/AmCH interfaces). Local relaxation of the PolyesternX/AmCH interfaces leads to significant increases in $W_{\text {sep }}(\sim 60-126 \%)$ and $V_{c}(\sim 51-123 \%)$ and decreases in $d_{0}(\sim 0.5-0.9 \AA)$, as compared to their rigid counterparts. The corresponding increases in $W_{\text {sep }}$ in the case of graphite were again comparably smaller. ${ }^{116}$

Clearly, the polyester models undergo significant reorganisation during minimisation. Reductions in $d_{0}$ suggest that for all interfaces examined, the polymers migrate toward the amorphous carbon surface, as also observed in the case of graphite. ${ }^{116}$ This leads to a significant increase in adhesion and contact volume at the interface. However, it appears that the extent of this reorganisation and its effects on interfacial adhesion differ significantly between graphite and amorphous carbon interfaces (i.e., differs according to the environment in which the polymer models are allowed to relax).

It can be seen from Table 4.2 that following minimisation, several of the modified polyesters now exhibit an increase in adhesive interaction with amorphous carbon, as compared to the minimised base polyester. In most cases, this is accompanied by a corresponding rise in contact volume $\left(V_{c}\right)$ and a reduction in $d_{0}$. The minimised Polyester7F and Polyester15F interfaces (Systems $2 b^{\text {min }}$ and $5 b^{\min }$ ), exhibit $\sim 7$ and $\sim 2 \%$ increases in $W_{\text {sep }}$, respectively, as compared to the minimised base interface. The equivalent carboxylated interfaces (Systems $4 b^{\text {min }}$ and $7 b^{\text {min }}$ ) lead to $a \sim 7$ and $8 \%$ increase in $W_{\text {sep }}$, respectively. In these instances, modifications that initially led to significant reductions in interfacial adhesion between polyester and carbon under a rigid regime, in fact promote interfacial adhesion following local relaxation. In contrast, for the minimised Polyester7OH and Polyester15OH surfaces, hydroxylation still leads to a reduction in adhesion to amorphous carbon $(\sim 3-4 \%)$; however, the magnitude of the 
reduction is smaller than that observed under a rigid regime. These results differ from the previously reported graphite interfaces, ${ }^{116}$ where low levels of all three modifications continued to provide some reduction in adhesion $(\sim 5-10 \%)$, following local relaxation.

For modified polyesters with higher concentration of surface substituents we observed a rather different trend. In particular, the minimised Polyester(25 x 3)F and Polyester75F interfaces exhibit $\sim 8$ and $\sim 19 \%$ reductions in $W_{\text {sep }}$ respectively, relative to the minimised base interface. These modified interfaces also display a reduction in interfacial contact volume $(\sim 8-20 \%)$ and an increase in $d_{0}(\sim 0.2-0.4 \AA)$. Under rigid constraints, the same systems were shown to lead to a $\sim 1-2 \%$ reduction in $W_{\text {sep }}{ }^{a p p}$. Likewise, the Polyester75OH and Polyester(25 x 3)OH models now lead to $2-6 \%$ reduction in adhesion with the amorphous carbon model, whereas under rigid constraints, the same systems exhibited a $\sim 1 \%$ increase and a $\sim 3 \%$ reduction, respectively. Therefore, as previously postulated ${ }^{116}$ high level functionalisation is required to observe a larger reduction in adhesion for the locally relaxed interface models.

Interestingly, atomic density values $\left(\rho_{2}\right)$ displayed in Table 4.2, do not correlate well with observed trends in $W_{\text {sep }}$. Moreover, for some polyester models, relaxation against the amorphous carbon surface results in an increase in atomic density, while for others, a reduction is observed. In comparison, relaxation of the polymer models against the graphite surface has generally been shown to result in an increase in the atomic density of the polymer structures, indicating an overall flattening or 'smoothing' of the surfaces (i.e., flattening of protruding substituents).

Table 4.2 also reveals that for some polymer/AmCH interfaces, there is a poor correlation between $d_{0}$ and $W_{\text {sep }}$. For example, the Polyester15OH/AmCH interface exhibits a weaker interfacial adhesion as compared to the base interface, yet displays a smaller $d_{0}$ value. The Polyester $15 \mathrm{CO}_{2} \mathrm{H} / \mathrm{AmCH}$ interface exhibits a significantly stronger interfacial adhesion than the Polyester7 $\mathrm{CO}_{2} \mathrm{H} / \mathrm{AmCH}$, yet the $d_{0}$ values for the former system is slightly larger.

Irregularities in $d_{0}$ and $\rho$ trends, indicate that there are variations in the degree of polymer surface reorganisation, depending on the chemical nature of the surface residues, the concentration of chemical modifiers, and the environment in which the 
polymer models are allowed to relax (i.e., the nature of the opposing carbon surface). However, these irregularities may also be a direct consequence of the definition used to determine the surface boundaries of the polymer models. For example, for the Polyester15OH/AmCH interface, the reference polymer bulk atom is protruding from the polymer surface and is in close proximity to the amorphous carbon surface boundary. Therefore, in this case, the interface exhibits a notably low $d_{0}$ value, irrespective of its relatively strong interfacial adhesion. Since the calculated $W_{\text {sep }}$ value is independent of the definition used to determine the surface boundaries of the polyesters, we will generally focus our attention to trends in interfacial adhesion.

During energy minimisation, the polyesters reorganise against the fixed carbon model, conforming to its surface profile. Structural irregularities at the interface are eliminated, leading to an increase in contact between surfaces, as indicated by the change in contact volume, $V_{c}$. This rise in interfacial contact is accompanied by a rise in interfacial adhesion $\left(W_{\text {sep }}\right)$. Since the irregular surface of the amorphous carbon provides a greater surface area than graphite, relaxation of the polymer against the former results in a larger interfacial contact area and a more significant increase in interfacial adhesion compared to the equivalent graphite system. A schematic representation of the reorganization occurring at both polymer/carbon interfaces is shown in Figure 4.4. The relationship between contact area and adhesion is thoroughly investigated by the experimental work of Israelachvili and co-workers, ${ }^{164}$ who note that the entanglement of surface segments at the interface, which is directly related to the real contact area, can contribute to higher interfacial adhesion energies. Mizes et al. ${ }^{52}$ used AFM to investigate the relationship between polymer adhesion and submicron surface roughness, and they too found adhesion to be dependent on the direct contact area between tip and sample. They showed that increasing the contact area between interacting surfaces results in an increased strength of adhesion.

The irregular topography of the amorphous carbon structure appears to also impact on the reorganisation of the substituents themselves. Concave areas along the amorphous carbon surface provide vacant regions into which local surface modifiers can relocate to minimise their overall repulsive interactions with both the carbon model and the bulk of the polyester. Bhattacharjee et al. ${ }^{175}$ investigated the interaction energy between rough 
surfaces by using a theoretical model based on the Derjaguin-Landau-Verwey-Overbeek theory. Their results showed that the vdW interaction energy between two rough surfaces is energetically favourable in a configuration whereby the protrusions from one surface intrude into depressions in the second surface. This is commonly referred to as interdigitation or interlocking. For the polyester/graphite interfaces, interdigitation is not apparent, as surface modifiers are confined between the bulk of the polyester and the flat surface of the graphite. This is not the case for the polyester/amorphous carbon interfaces and as a consequence, the energy minimised Polyester7X and Polyester15X surfaces presented here, do not exhibit the notable reductions in adhesion observed previously with graphite.

Rigid Configurations

(a)
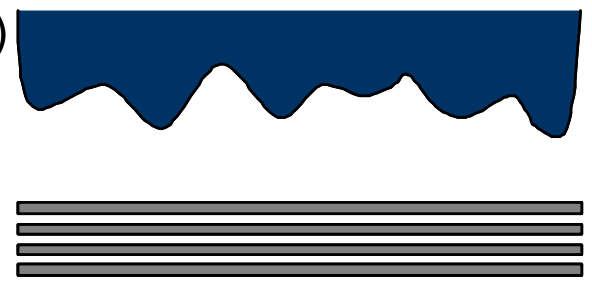

graphite (b)
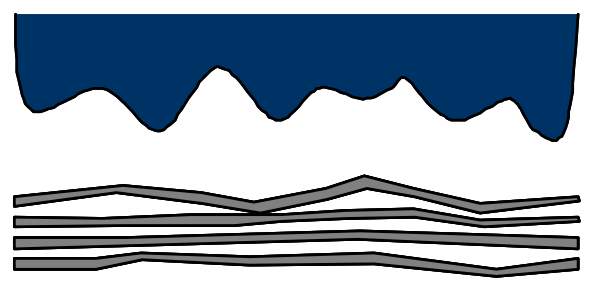

amorphous carbon

Relaxed Configurations

(c)

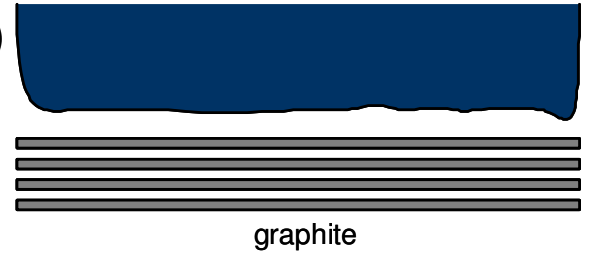

(d)

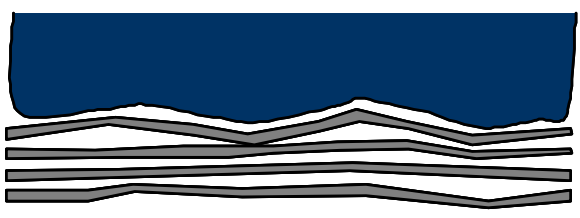

amorphous carbon

Figure 4.4 Schematic representation of the effects of relaxation on polyester/carbon interfaces. Relaxation against the graphite structure, results in the smoothing of the polymer coating. Relaxation against the amorphous carbon model, leads to interfacial interlocking. In both of these examples, structural irregularities at the interface are effectively eliminated.

To help determine just how effective our nanoscale surface modifications are under an energy minimised regime, we evaluated the chemical and structural energy contributions for each modified, energy minimised interface. The chemical contribution $\Delta W_{\text {sep }}{ }^{\text {chem }}$, provides a measure of the differences in adhesion values among the modified coatings, that arise from changes in the chemical constitution of the surfaces rather than from changes in the configuration of the surfaces. Positive values of $\Delta W_{\text {sep }}{ }^{\text {chem }}$, indicate 
an increase in the strength of interaction between carbon contaminant and polyester, while negative values indicate a reduction in the interaction strength. The structural contribution $\Delta W_{\text {sep }}^{\text {struc }}$ provides a measure of the difference in adhesion values arising solely from changes in the configuration of the coatings. Positive values of $\Delta W_{\text {sep }}$ struc signal an increase in strength of the interaction between polymer and carbon while negative values signal a reduction in the interaction strength. Both parameters were obtained by evaluating the work of separation for the hydrogenated interfaces $\left(W_{\text {sep }}{ }^{H}\right)$. The hydrogenated surfaces are functionalised polymers whose modifications have been essentially removed and replaced with hydrogen atoms. For a full description of these parameters refer to Section 4.3.2.

Table 4.2 reveals that the work of separation values for the hydrogenated interfaces $\left(W_{\text {sep }}{ }^{H}\right)$ are smaller than for their corresponding functionalised systems $\left(W_{\text {sep }}\right)$. Isolation of the chemical effects $\left(\Delta W_{\text {sep }}^{\text {chem }}\right)$ indicates for the minimised systems, the substituents contribute to a net increase in adhesion i.e., the effective residue-carbon interaction is attractive for all surface types and levels of chemical modification. This is an important observation, as it highlights the fact that protruding surface modifiers reorganise to energetically favourable regions along the polymer surface, resulting in a reduction in the repulsive interactions and/or an increase in attractive interactions at the interface.

It can be seen from Table 4.2 that the magnitude of $\Delta W_{\text {sep }}^{\text {chem }}$ rises, with increasing level of modification, suggesting that for polyesters with relatively high surface concentration of modifiers, the residue-carbon attraction is stronger compared to their low-level modified equivalents. In simple terms, the magnitude of $\Delta W_{\text {sep }}^{\text {chem }}$ is larger for these highly modified systems due to the same saturation effect we observed in Procedure A. Furthermore, $\Delta W_{\text {sep }}{ }^{\text {chem }}$ is shown to be dependent on the chemical nature of the substituents, with hydroxylated and carboxylated interfaces exhibiting a stronger residue-carbon interaction $\left(\Delta W_{\text {sep }}^{\text {chem }}\right)$ compared to their fluorinated counterparts. This is to be expected, as oxygen atoms in hydroxyl and carboxyl functional groups exhibit a stronger dispersive interaction with aromatic carbon relative to fluorine (Figure 4.2).

In the case of the structural component $\left(\Delta W_{\text {sep }}{ }^{\text {struc }}\right)$, Table 4.2 shows that for the majority of modified systems $\Delta W_{\text {sep }}^{\text {struc }}$ is negative, indicating that for these interface systems, the structural conformation of the polymer leads to reduced interfacial 
adhesion. In particular, for highly functionalised interfaces (Systems $8 b^{\text {min }}$ to $11 b^{\text {min }}$ ), variations in the structural conformation of the underlying polymer lead to significant reductions in adhesion $(\sim 12-23 \%)$, while for the Polyester7OH and Polyester15OH interfaces, they lead to reductions of only $\sim 5-6 \%$. Hence, the high density of the substituents $\left(4.7\right.$ residues per $\mathrm{nm}^{2}$ ) appears to help retain greater rigidity at the surface of the polymer, as compared to lower levels of surface coverage ( 0.4 or 0.9 residues per $\left.\mathrm{nm}^{2}\right)$, counteracting the previous chemical effects. The structural component $\left(\Delta W_{\text {sep }}{ }^{\text {struc }}\right)$ also differs according to the chemical nature of the functional groups introduced to the polyester surface. For the Polyester7OH system, $\Delta W_{\text {sep }}$ struc leads to a reduced adhesion, while for the equivalent carboxylated and fluorinated systems, it leads to increased adhesion, indicating that hydroxyl surface modifiers retain greater surface disorder and rigidity than fluorine or carboxyl residues. This also appears to be the case for the Polyester15X systems but is not particularly evident for highly functionalised interfaces (Polyester(25 x 3)X and Polyester75X interfaces).

To quantify the overall observations, a scale representation of each PolyesternX/AmCH system in its rigid and energy minimised configuration is presented in the following page, Figure 4.5. Illustrated graphically in the diagram, are the $d_{0}$ values for each of the modified interfaces, along with the average separation of the seven highest substituents and average separation of the remaining surface modifiers from the amorphous carbon boundary.

From Figure 4.5, the level of fine-scale roughness of the various modified polyester models can be approximated by the position of the highest substituents, relative to the $d_{0}$ value of the interface (i.e., the separation of the seven highest modifiers from the predetermined polyester surface boundary). For rigid interfaces (Systems $N b^{\text {rig }}$ ), the highest substituents are located at a separation of $\sim 1.5-2.2 \AA$ from the polymer boundary. 


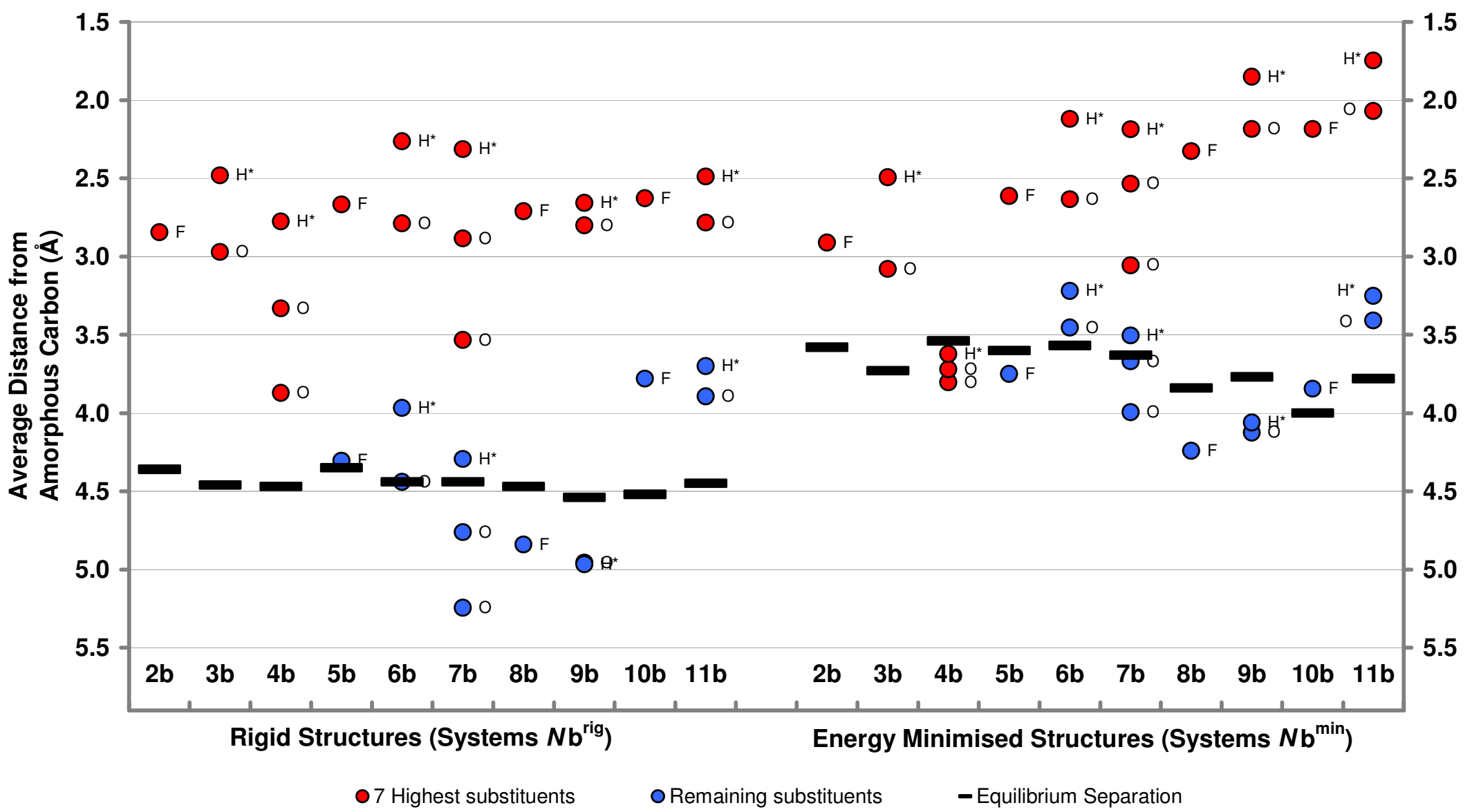

Figure 4.5 Scale representation of the average separation between amorphous carbon and the polyester substituents, and the equilibrium separation, for rigid and energy minimised systems. 
In comparison, Figure 4.5 shows that following minimisation, the highest substituents are positioned slightly closer to the polyester surface boundary, at a distance of 0.7-2.0 $\AA$ away from the polymer indicating a certain degree of surface flattening or compression. This surface flattening is primarily a result of the overall migration of the underlying polymer toward the carbon surface (reductions in $d_{0}$ ) rather than the movement of surface residues away from the carbon surface. In fact, we have observed that several substituents actually move away from the polymer boundary (toward the amorphous carbon surface) because the presence of cavities along the carbon surface provides vacant regions into which the local surface atoms (including substituents) can relax. Nonetheless, the physical effect of accentuated fine-scale roughness that helps reduce the effective contact between interacting surfaces (polyester and amorphous carbon) is diminished by relaxation. Moreover, the repulsive interaction of protruding modifiers with the amorphous carbon surface is diminished due to local repositioning of substituents. Taken as a whole, the configuration of the minimised PolyesternX/AmCH interface, results in a significantly stronger interfacial adhesion compared to their equivalent rigid structure.

We have shown that the degree of polymer surface reorganisation is dependent on the chemical nature of the surface residues and the total number of functional groups affixed at the surface of the polyester (i.e., on the surface density of the modifiers). It can be seen from Figure 4.5 that the minimised Polyester7OH model (System $3 b^{\text {min }}$ ) maintains a certain degree of atomic roughness along its surface as compared to its fluorinated or carboxylated equivalents (Systems $2 b^{\min }$ and $4 b^{\min }$, respectively), i.e., the average distance from the seven highest $\mathrm{OH}$ groups to the predetermined polyester boundary $\left(d_{0}\right)$ is greater than that exhibited by the $\mathrm{F}$ or $\mathrm{CO}_{2} \mathrm{H}$ residues. It can be suggested that due to the smaller overall size of fluorine substituents as well as the observed flattening of the acid groups (best illustrated by System $4 b^{\text {min }}$, Figure 4.5) they do not produce the same surface roughness effects as the $\mathrm{OH}$ substituents. In the case of acid groups $(\mathrm{COOH})$, the carboxylic $\mathrm{C}-\mathrm{OH}$ groups show significant movement toward the bulk of the polyester, while the carboxylic $\mathrm{C}=\mathrm{O}$ oxygens show a slight movement away from the bulk (i.e., toward the carbon surface). In accordance with this observation, Inagaki et al. ${ }^{154}$ noted that relaxation of plasma modified PET surfaces 
under ambient conditions where characterised by the movement of the newly formed C$\mathrm{O}$ groups away from the topmost layer and to the movement of $\mathrm{C}=\mathrm{O}$ groups in the ester residues towards the topmost layer.

As we have already demonstrated, the observed local rearrangements of substituents causes variations in surface roughness, which can be directly related to the differences in adhesive strength between polyester and amorphous carbon. Figure 4.5 shows that following relaxation, protruding surface modifiers for the Polyester15X models (Systems $5 b^{\mathrm{min}}$ to $7 \mathrm{~b}^{\mathrm{min}}$ ) are located further from the polymer boundary as compared to the equivalent Poylester7X interfaces (Systems $2 b^{\text {min }}$ to $4 b^{\text {min }}$ ), indicating an increase in atomic roughness. For the Polyester75X and Polyester(25 x 3)X interfaces (Systems $8 \mathrm{~b}$ to $11 \mathrm{~b}$ ), this increase in atomic roughness is further enhanced, as the average distance from the seven highest groups to the polyester is even greater than that exhibited by their Poylester15X counterparts. Not surprisingly, the driving force associated with the local relaxation of high-density surface residues is more demanding as compared to relaxation of low-density surface modifiers. This is in agreement with the observed variations in $\Delta W_{\text {sep }}^{\text {struc }}$. We compare these results with an experimental study conducted by Selli et al. ${ }^{39}$ reporting on the stability of $\mathrm{SF}_{6}$ plasma treated PET surfaces. They observed that the samples treated under relatively high radio frequency power exhibited a greater amount of fluorine content immediately after treatment as compared to the lowfrequency treated samples. These same samples also exhibited a smaller decrease in the $\mathrm{F} / \mathrm{C}$ ratio during storage in humid air and maintained greater hydro-repellence properties than the less modified low-RF treated samples. Kim et al. ${ }^{40}$ reported on the durability of hydrophobic improvement of polymer surfaces, achieved by plasma source ion implantation. They showed that the long-term stability of the hydrophobic surfaces improves with increased fluorine content. Similarly, we have shown that the highly fluorinated surfaces are more rigid than their equivalent low functionalised models, maintaining more atomically rough surface profiles following relaxation, and would therefore be expected to retain greater hydrophobicity.

Surfaces which maintain an atomically rough morphology would be expected to exhibit a relatively weak adhesion with amorphous carbon. However, due to the overall attractive residue-carbon interaction $\left(\Delta W_{\text {sep }}{ }^{\text {chem }}\right)$ for the minimised systems, these 
structural effects on adhesion are partially offset. This is also illustrated graphically in Figure 4.5 by the average separation of the deeper modifiers (remaining substitutions). It can be seen that following minimisation, the average separation of the deeper substituents from the model carbon surface is $\sim 3.2-4.2 \AA$. In agreement, analysis of the force-field parameters (see Figure 4.2) reveals that the effective vdW interaction between aromatic carbon and substituent atom types $(\mathrm{C}, \mathrm{H}, \mathrm{O}$, and $\mathrm{F})$ is attractive for inter-atomic separations larger than $\sim 3.2 \AA$. Also, due to the notably strong vdW attraction between oxygen and aromatic carbons, this saturation effect is particularly pronounced for surfaces with the highest number of hydroxyl residues (Polyester $(25 \mathrm{x}$ 3) $\mathrm{OH}$ and Polyester75OH). Consequently, these models do not exhibit the notable reductions in adhesion to amorphous carbon, demonstrated by their fluorinated counterparts (Polyester(25 x 3)F or Polyester75F), or by the low-level hydroxylated surface (Poylester7OH).

An additional factor that also needs to be taken into account, particularly for the Polyester $75 \mathrm{OH}$ and Polyester $(25 \times 3) \mathrm{OH}$ models, is that there is an added complexity of intramolecular hydrogen bonding. Hydroxyl groups located at the outer-surface of these polymers can form hydrogen bonds with adjacent polar species. This is shown in the next page (Figure 4.6), where we have taken a snapshot of the minimised Polyester75OH/AmCH interface and highlighted the possible regions of hydrogen bonding. The hydrogen bond criterion was defined by a hydrogen-acceptor distance of $2.5 \AA$ or less, and a hydrogen bond angle of $90^{\circ}$ or more. The hydrogen bond angle was measured between the donor-hydrogen and hydrogen-acceptor vectors. It can be seen from this image that hydroxyl groups on the surface can form hydrogen bonds with adjacent polar species, resulting in the flattening of the hydrogen atoms of the $\mathrm{OH}$ residues. The image shows a significant number of hydrogen bonds formed simply due to the availability of hydrogen bonding pairs. However, when the same criteria were used to check for hydrogen bonding in the minimised Polyester7OH/AmCH, only one possible hydrogen bond was observed. ${ }^{108}$ 


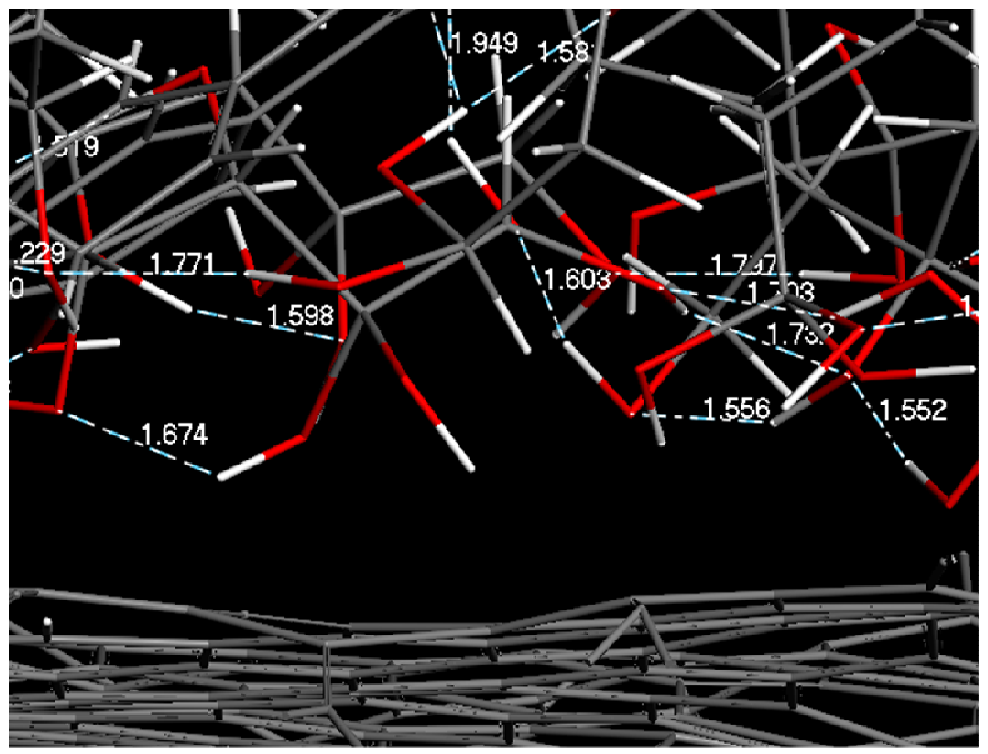

Figure 4.6 Snapshot of energy minimised Polyester75OH/AmCH interface, highlighting the possible hydrogen bonding regions and with the inclusion of donor-acceptor distances in Å.

We have also observed that the flattening due to hydrogen bonding is more probable for the Polyester75OH surface as compared to the Polyester(25 x 3) OH model. This is because for the Polyester75OH surface, the functional groups are distributed more uniformly across the upper region of the polyester surface, whereas for the Polyester(25 $\mathrm{x} 3) \mathrm{OH}$ model, the $\mathrm{OH}$ groups are more localized and are distributed across deeper layers of the surface. This may help explain why the flatter surface (Polyester75OH) leads to a lower value of $\Delta W_{\text {sep }}^{\text {struc }}$ (i.e. makes a weaker contribution to the overall reduction in interfacial adhesion). Such flattening effects are not present in the fluorinated systems, and therefore, Polyester75F exhibits a greater atomic surface roughness than both Polyester $75 \mathrm{OH}$ and Polyester $(25 \times 3) \mathrm{OH}$, thus leading to the weakest observed adhesion with carbon.

Overall, these observations highlight the additional complexity arising from hydrogen bonding, which can affect the degree of surface reorganisation and the adhesive strength between polyester and amorphous carbon surfaces. In agreement with these findings, several experimental studies have shown that specific interactions such as hydrogen bonding play an important role in controlling the physical and chemical surface properties of a material. ${ }^{176-178}$ Kuo et al. ${ }^{176}$ found that by increasing the intermolecular hydrogen bonding of co-polymers (i.e., increasing the hydrogen bonding 
between polymer chains) one can increase the surface free energy. Similar studies have also shown that increasing the degree of intermolecular hydrogen bonding of a polymeric material tends to increase its surface energy. ${ }^{178,179}$ This rise in surface free energy is more often than not, accompanied by a reduction in the degree of surface roughness (i.e. a flattening of the surface protrusions as we have observed in the case of the Polyester75OH model). An example of this worth highlighting is work undertaken by Sun et al. ${ }^{177}$ who investigated the reversible switching between superhydrophobicity and superhydrophilicity, of a poly(N-isopropylacrylamide) (PNIAPAAm) surface. They found that at temperatures below $\sim 33^{\circ} \mathrm{C}$, intermolecular hydrogen bonds between polymer chains become dominant, lowering the surface free energy, reducing the level of surface roughness and rendering the polymer hydrophilic.

\subsection{Conclusion}

The interfacial adhesion of modified polyester and amorphous carbon surfaces was investigated using force-field techniques. Modifications include the addition of a varying number of hydroxyl, carboxyl, or fluorine groups. Adhesion calculations between rigid surfaces indicate that accentuated atomic surface roughness, caused by protruding modifiers, significantly reduces adhesion with amorphous carbon. Rigid and atomically rough polyester surfaces require only a small number of modifiers for optimal adhesive resistance to carbon.

To approximate relaxation of the polyester surface, the energy minimisation technique was employed. Relaxation of the polyester surfaces against the carbon structure leads to a significant increase in interfacial adhesion. During energy minimisation, surface modifiers reorganise to energetically favourable regions along the polymer surface. The degree of reorganisation is strongly dependent on the structural topography of the carbon model and also the chemical type and surface concentration of functional groups initially introduced to the polymer surface. Highly functionalised surfaces appear to be more rigid than their equivalent low-level functionalised counterparts and hence maintain more atomically rough surface profiles, which should contribute to lower adhesion. However, high-level modification is also accompanied by a significant increase in the dispersive chemical contribution to the work of separation, 
which largely cancels the effects of increased atomic roughness. Nevertheless, under this locally relaxed regime, the presence of surface modifiers is still predicted to reduce adhesion between polyester and amorphous carbon. In the next chapter of this study we attempt to achieve fully relaxed states across all functionalised systems including graphite and amorphous carbon based interfaces, by employing molecular dynamics. This will also enable us to further explore the aging process of these modified polyester surfaces. 


\section{Chapter 5}

\section{Effects of Aging on Adhesion between Polyester and Carbon}

\subsection{Overview}

In this section we will use classical molecular dynamics to examine how aging of functionalised polyester surfaces perturbs the adhesive strength at the polymer and carbon interface. We will examine an array of polymer/carbon interfaces, consisting of fluorine, carboxyl and hydroxyl treated polyesters combined with amorphous carbon as well as graphite surface contaminant models. In this section, we will show that the energy minimisation technique used in the previous chapter provides only a 'snapshot' of the initial stages of the relaxation process. In comparison, molecular dynamic simulations achieve further restructuring of the interfaces, resulting in the systems converging to similar values of interfacial adhesion and separation. We will demonstrate that during molecular dynamics, carbon moieties from the bulk of the polymer migrate towards the boundary of the film and in close proximity to the carbon surface analogous to hydrophobic recovery. Meanwhile surface substituents continue to reorientate and move away from the interface towards the bulk polymer. We will demonstrate that for surface modification to be effective, i.e., reduce interfacial adhesion with carbon, the polyester surfaces need to maintain both their rigidity and atomic roughness. 


\subsection{Introduction}

Methods of treating polyester surfaces such as UV/ozone, and plasma modification produce chemical groups on the surface which altar the surface properties of the original polymer films. While the surface properties are altered immediately after treatment, the use of such methods is hindered by the effects of aging. This is process whereby treated surfaces recover their primitive properties through the reorganisation of surface polymer chains, tending to reduce the interfacial tension with the opposing phase (for more detail, refer to Section 1.4). The induced modification of the surface is therefore not permanent as the surface tends to recover to the untreated state during storage. The reversibility of surface modification is known consequence of the fact plasma and UV/ozone surface treatments modify a very thin (a few nanometres) polymer surface layer, resulting in instability of the induced chemical groups. Aging is a complicated process with surface stability dependent on many factors such as molecular weight of the surface chemical groups, environment of storage, strength of interaction between bulk and surface, and the method of initial modification.

Several early studies ${ }^{180-182}$ have investigated the effects of aging on modified poly(ethylene terephthalate) (PET) surfaces. Modification of the PET films was undertaken either by UV/ozone or by corona discharge methods to improve wettability. Results showed that during storage, the migration/reorientation of low-molecularweight-oxidised species from the surface of the polymer towards the bulk, leads to a rapid increase in contact angle and recovery of the untreated film's surface properties. The reason for the formation of low-molecular-weight species is due to polymer chain scission (fragmentation of polymer chains) occurring during modification, and the general belief is that such species exhibit significant mobility due to their small size and low molecular weight. But even in these early studies, evidence indicates that aging is a complex process, which involves a combination of migration/reorientation of lowmolecular-weight species, reorientation of high-molecular-weight species and migration or diffusion of species from the bulk to the surface.

In a more recent study, ${ }^{39}$ the effect of storage environment on various fluorine modified polyesters was investigated. The plasma-treated films were stored in humid air 
as well as water, and their fluorine surface composition and water-repellency stability was monitored during storage. The results presented indicate that fluorinated polyesters are also susceptible to aging in humid air or water. The surface tension between the polymer and the contacting phase is reduced with the migration or folding of the hydrophobic polymer moieties toward the inside of the polymer.

Riccardi et al. ${ }^{160}$ treated polyester fabrics using plasma, with the aim of permanently increasing their hydrophilicity. Water adsorption on the fabrics did in fact improve initially with treatment due to the introduction of oxygen-enriched polar groups. The treated surfaces were subsequently stored under air and water for 10 days and in both of these environments, the modified fabrics exhibited a reduction in the oxygen surface content, i.e., the treated polyester surfaces underwent hydrophobic recovery in both hydrophobic and hydrophilic aging environments. In the case of air-storage, the aging was attributed to folding of surface polymer chains and reorientation of polar groups away from the surface, tending to decrease interfacial tension. In the case of waterstorage though, oxygen-containing polar moieties would be expected to preferentially remain at the interface in contact with polar water. The explanation given for this observed phenomenon was attributed in part to the fact that the oxygen present on the surface was in fact low-molecular-weight-oxidised species which could be easily dissolved by water, and thus removed from the treated polymer surface.

Several researchers have succeeded in creating stable plasma-modified polyester surfaces. In a recent study by Jacobs et al., ${ }^{183}$ plasma modification of PET films was undertaken in a $95 / 5 \% \mathrm{He} / \mathrm{CF}_{4}$ mixture at sub-atmospheric pressure. This treatment resulted in an improvement in the hydrophobicity of the film, as indicated by contact angle measurements, due to incorporation of fluorine-containing functional groups. After treatment, the films were stored for up to 20 days to examine the effects of aging on the plasma-treated surfaces. Results showed that the contact angle did not change during storage, but remained stable indicating permanent hydrophobic improvement. Similarly, Kim et al. ${ }^{40}$ modified polyester films such as PET using plasma-source-ion-implantation (PSII) to improve water repellency. XPS analysis revealed that PSII treatment resulted in the incorporation of fluorinated compounds such as $\mathrm{CF}_{3}$ and $\mathrm{CF}_{2}$ to the surface of the polymer, increasing its hydrophobicity. Examination of aging effects shows that PSII 
treatment results in a stable modified surface layer that remains unaffected during storage. This stability is believed to be as result of the large number of functional groups introduced to the surface as well as the significant thickness of the modified layers. From the above literature and our results from the previous section, it appears that high level functionalisation may help to reduce the mobility of the polymer chains at the surface.

There are various chemical reactions and physical alterations that also occur during modification of polymeric surface resulting in polymer chain scission, etching, crosslinking, post-treatment reactions and changes in glass transition temperature (Tg). Such alterations further complicate a complete understanding of the aging process. Research aimed at elucidating these issues is still continuing. Here, we employ classical molecular dynamic simulations to model the effects of aging modified polyester surfaces against carbon structures. We investigate how physical and chemical surface changes that occur during aging can affect the adhesion strength at the polymer/carbon interface.

\subsection{Molecular Models and Theoretical Procedures}

\subsubsection{Composition of Molecular Models}

The untreated polyester surface (polyester) and modified polyesters (PolysternX), were constructed as described previously (Chapter 3.1). In this section, two models were used to simulate the carbon contaminant surfaces: (a) graphite; (b) amorphous carbon. The graphite model, comprising of six ideally flat graphitic (001) layers ${ }^{138}$ is used to model an ideal soot-particle surface. ${ }^{135-137}$ The amorphous carbon model (AmCH) which consists of six buckled graphite-like sheets, is consistent on a nanoscale with the surface of amorphous industrial char, ${ }^{139}$ and due to its irregular surface topography, represents a realistic tar-ball surface. A detailed description of the carbon models has also been included in Section 3.3.

A total of 22 interfaces were formed by combining each polymer surface with each carbon surface. Two base systems, polyester/graphite and polyester/AmCH, were constructed by combining the untreated polymer with graphite and amorphous carbon, respectively. The modified interfaces, denoted as PolyesternX/graphite and PolyesternX/AmCH, were constructed by combining each treated polymer with the 
respective carbon models. Each system was enclosed in a unit cell with dimensions $40 \AA$ $\times 40 \AA \times 300 \AA$. Extension of the cell in the $\mathrm{z}$ direction ensures that adjacent interfaces do not interact along the z-axis, when periodic boundary conditions are applied. As a result, infinite $2 \mathrm{D}$ surfaces in contact were simulated. We have included an illustrative example of a polyester/AmCH interface (Figure 3.4).

\subsubsection{Theoretical Procedures}

Adhesion calculations were performed and interfacial parameters describing the interactions between the carbon and polyester surfaces were determined (see Section 3.4). We also evaluated the chemical and structural contribution to the adhesion strength at the interface, $W_{\text {sep }}^{\text {chem }}$ and $W_{\text {sep }}^{\text {struct }}$ respectively, through the process described in Section 4.3.1. In the previous chapter we used energy minimisation to approximate surface relaxation. Here, relaxation of the surface was undertaken by performing molecular dynamics simulations on each minimised interface structure in the NVT ensemble, for $1 \mathrm{~ns}$, with the temperature maintained at $400 \mathrm{~K}$ (above the $\mathrm{Tg}$ of the polyester) using the Andersen thermostat. ${ }^{131}$ This molecular dynamics procedure leads each structure to equilibrium and to a steady value of adhesion energy. The resulting polymer/graphite and polymer/AmCH systems are termed relaxed structures and denoted Systems $N \mathrm{a}^{\text {rel }}$ and $\mathrm{Nb}^{\text {rel }}$, respectively. We note here that, for selected systems, molecular dynamics simulations were also performed for up to $4 \mathrm{~ns}$; however, in these instances, no further change in adhesion values was observed. Therefore, we believe that the trends observed after $1 \mathrm{~ns}$ reflect the true trends for polymer aging. The atom-based non-bond summation method was used for molecular dynamics simulations to retain computational tractability with a cut-off distance of $15.5 \AA$ and a long-range tail correction. At the completion of molecular dynamics simulations, energy minimisation was performed on the system with the conjugate gradient procedure at a convergence criterion of $0.04 \mathrm{~kJ} \mathrm{~mol}^{-1}$, and the Ewald summation method to 'truncate' non-bonded interactions at a convergence of $4 \times 10^{-4} \mathrm{~kJ} \mathrm{~mol}^{-1}$. It is important to note here that the two carbon models were kept fixed in their original geometry during relaxation procedures, to maintain their structural integrity. The boundaries of the models were defined as discussed in Section 3.5. 


\subsection{Results and Discussion}

Variations in adhesion strength at the polymer/carbon interface arise from variations in the level of relaxation (minimisation or molecular dynamics), the nature and degree of polyester surface modification, and the chemical and physical surface properties of the carbon model in question. To elucidate the factors involved, we present the results for the Polyester/graphite interfaces first, followed by the results for the Polyester/AmCH interfaces and, finally, end with a discussion comparing and contrasting these results and their relation to experimental aging studies of modified polyester surfaces in the literature.

\subsubsection{Polyester and Graphite Interfaces}

Key interaction parameters for the relaxed Polyester/graphite interfaces (Systems $\mathrm{Na}^{\text {rel }}$ ), are presented in the following page, Table 5.1. These include the work of separation $\left(W_{\text {sep }}\right)$, equilibrium interfacial separation $\left(d_{0}\right)$, interfacial contact volume $\left(V_{c}\right)$, work of separation for hydrogenated interfaces $\left(W_{\text {sep }}{ }^{H}\right)$, chemical contribution $\left(W_{\text {sep }}{ }^{\text {chem }}\right)$, and structural contribution $\left(W_{\text {sep }}^{\text {struc }}\right)$, The effect of the aging process is reflected by the changes in these key interactions parameters from those of the rigid pre-aged interfaces (Systems $\mathrm{Na}^{\text {rig }}$ ) from our previous study. ${ }^{116}$

It is clear from Table 5.1 that, following relaxation, there is a significant increase in interfacial adhesion for the base system (polyester/graphite). This is reflected in a $\sim 106 \%$ increase in the work of separation value, relative to that attained under a rigid regime $\left(W_{\text {sep }}{ }^{a p p}\right)$. In conjunction, there is a corresponding decrease in the equilibrium separation $(\sim 0.3 \AA)$ and rise in interfacial contact volume ( $\sim 88 \%)$. In comparison, the local relaxation procedure led to an increase in theoretical adhesion $\left(W_{\text {sep }}\right)$ of only $\sim 79 \%$.

These same trends are also observed for the modified systems (PolyesternX/graphite interface). Full relaxation of the Polyester $n \mathrm{X} /$ graphite interfaces leads to significant increases in $W_{\text {sep }}(\sim 102-159 \%)$ and $V_{c}(\sim 89-170 \%)$ and decreases in $d_{0}(\sim 0.5-0.8 \AA)$, compared to their rigid counterparts. The increases in interfacial adhesion observed with the local relaxation procedure (energy minimisation) were again comparably smaller for these modified structures. ${ }^{116}$ 
Table 5.1 Adhesion Parameters for Polyester/Graphite Interfaces (Systems Na).

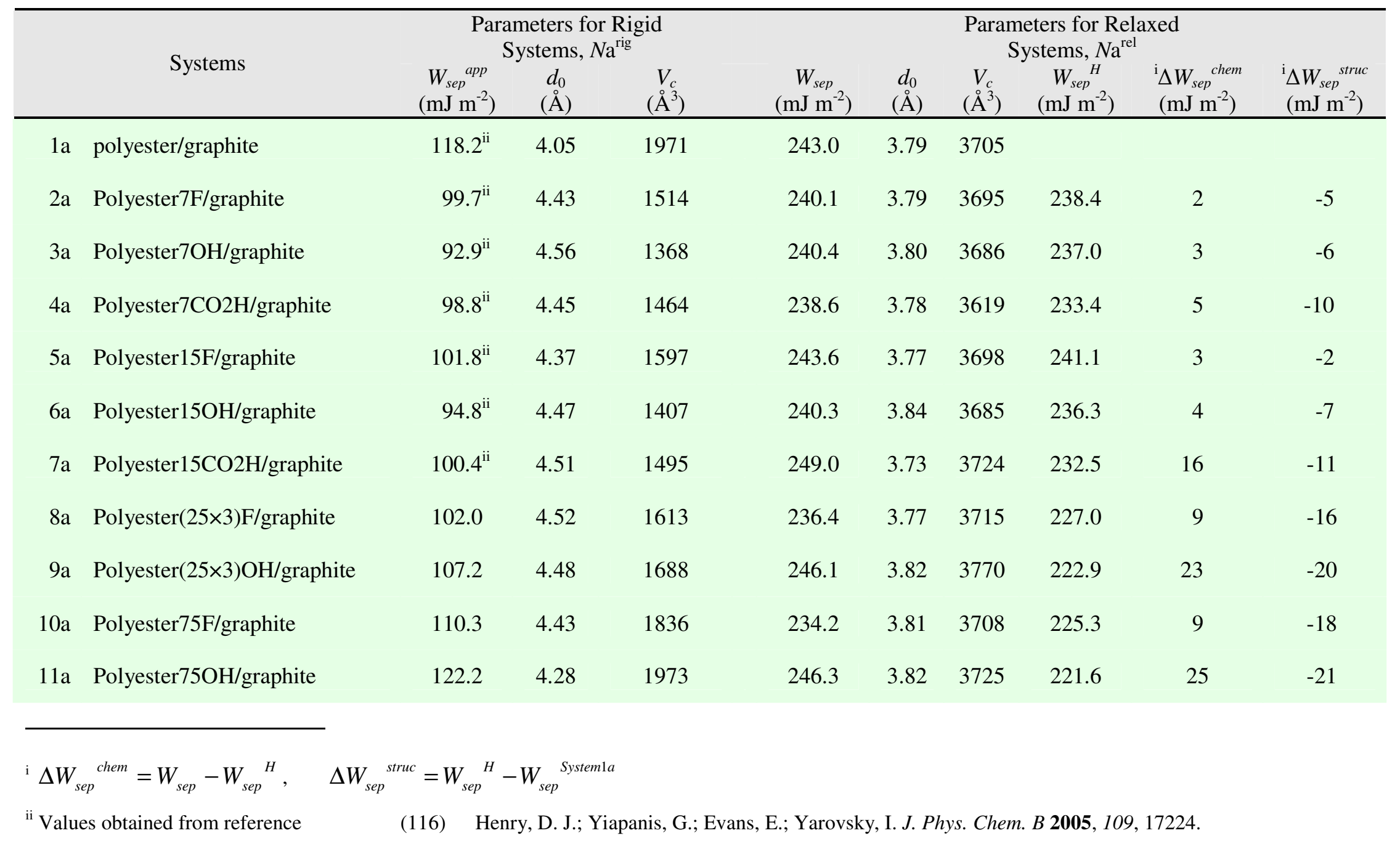


These initial findings suggest that the energy minimisation procedure implemented in the previous chapter provides only a "snapshot" of the initial stages of the relaxation process, involving local repositioning of constituents at the interface. In contrast, extensive MD simulations are necessary to produce changes that can be related to aging of the polyester surface.

One of the most significant features of the aging of these interfaces (Polyester/graphite) is the fact that the adhesion parameters $\left(W_{s e p}, d_{0}\right.$, and $\left.V_{\mathrm{c}}\right)$ of all modified systems appear to be converging toward the corresponding values for the unmodified system. This is demonstrated by the narrower range in the interaction parameter values of the fully relaxed PolyesternX/graphite interfaces $\left(\Delta W_{\text {sep }} \sim 15 \mathrm{~mJ} \mathrm{~m}^{-2}, \Delta d_{0} \sim 0.1 \AA\right)$ than was observed with the rigid interfaces $\left(\Delta W_{\text {sep }}{ }^{a p p} \sim 29 \mathrm{~mJ} \mathrm{~m}^{-2}, \Delta d_{0}{ }^{\text {app }} \sim 0.5 \AA\right)$. Low level functionalised polyesters (Polyester7X and Poylester15X), which under a rigid regime offered the greatest reductions in adhesion, are now found to have little or no effect on the work of separation ( $20-2 \%)$. High levels of hydroxylation (Poylester75OH and Polyester $(25 \times 3) \mathrm{OH})$ lead to marginal increases in adhesion $(\sim 1 \%)$, while high level fluorinated surfaces (Polyester75F and Polyester(25×3)F) exhibit slight reductions in adhesion $(\sim 3-4 \%)$.

As with the previous chapter, to help determine just how effective our nanoscale surface modifications are under a relaxed regime, we evaluated the chemical and structural energy contributions for each modified, relaxed interface. The chemical contribution $\Delta W_{\text {sep }}^{\text {chem }}$, provides a measure of the differences in adhesion values among the modified coatings, that arise from changes in the chemical constitution of the surfaces rather than from changes in the configuration of the surfaces. Meanwhile, the structural contribution $\Delta W_{\text {sep }}^{\text {struc }}$ provides a measure of the difference in adhesion values arising solely from changes in the configuration of the coatings. Both properties were obtained by evaluating the work of separation for the hydrogenated interfaces $\left(W_{\text {sep }}{ }^{H}\right)$. The hydrogenated surfaces are functionalised polymers whose modifications have been essentially removed and replaced with hydrogen atoms. For a full description of these parameters refer to section 4.3.2.

Table 5.1 reveals that the work of separation values for the "hydrogenated" interfaces $\left(W_{\text {sep }}{ }^{H}\right)$ are generally smaller than those for the corresponding functionalised 
systems $\left(W_{\text {sep }}\right)$. Isolation of the chemical effects $\left(\Delta W_{\text {sep }}{ }^{\text {chem }}\right)$ indicates that, in the relaxed interfacial configuration, the polymer substituents generally contribute to a net increase in adhesion $(\sim 1-10 \%)$; i.e., the effective residue-graphite interaction is attractive for all surface types and levels of chemical modification. For the Polyester7X and Polyester15X surfaces, the effective residue-graphite interaction increases interfacial adhesion by $\sim 1-7 \%$, while the $\Delta W_{\text {sep }}^{\text {chem }}$ values for Polyester $75 \mathrm{X}$ and Polyester(25×3)X systems indicate a $\sim 4-10 \%$ increase. Therefore, for highly modified surfaces, the dispersive interaction between the residues and graphite surface results in a stronger attraction at the interface compared to low level modified systems (Polyester7X and Polyester15X).

Table 5.1 shows that the structural effects for the PolyesternX/graphite systems lead to a $\sim 1-9 \%$ reduction in interfacial adhesion. For low level modified surfaces (in systems 2a-7a), the magnitude of this reduction $(\sim 1-4 \%)$ is smaller than that exhibited by highly functionalised surfaces ( 7-9\%). Hence, the high density of the substituents (4.7 residues $/ \mathrm{nm}^{2}$ ) appears to significantly restrict relaxation, relative to lower levels of surface coverage. However, the chemical interaction of the residues with graphite $\left(\Delta W_{\text {sep }}{ }^{\text {chem }}\right)$ generally offsets reductions observed as a result of the structural effects.

\subsubsection{Polyester and Amorphous Carbon Interfaces}

Key interaction parameters for the rigid and fully relaxed Polyester/amorphous carbon interfaces are presented in the following page, Table 5.2. It is clear that relaxation of these interfaces generally leads to the same qualitative trends observed for the Polyester/graphite systems. In particular, there are significant increases in $W_{\text {sep }}$ for both the base and modified interfaces ( 115-150\%), relative to their equivalent rigid systems. There are also increases in contact volume ( 100-138\%) and reductions in equilibrium interfacial separation $(\sim 0.7-1.0 \AA)$ following relaxation of these systems. The corresponding changes in $W_{\text {sep }}, V_{c}$, and $d_{0}$ observed with the energy minimisation were $\sim 60-126 \%, \sim 51-123 \%$, and $\sim 0.5-0.9 \AA$, respectively. In addition, the range of interaction parameters exhibited by the fully relaxed amorphous carbon interfaces $\left(\Delta W_{\text {sep }} \sim 15 \mathrm{~mJ}\right.$ $\mathrm{m}^{-2}, \Delta d_{0} \sim 0.2 \AA$ ) is also comparable to that observed for the relaxed graphite systems. 
Table 5.2 Adhesion Parameters for Polyester/Amorphous Carbon Interfaces (Systems Nb).

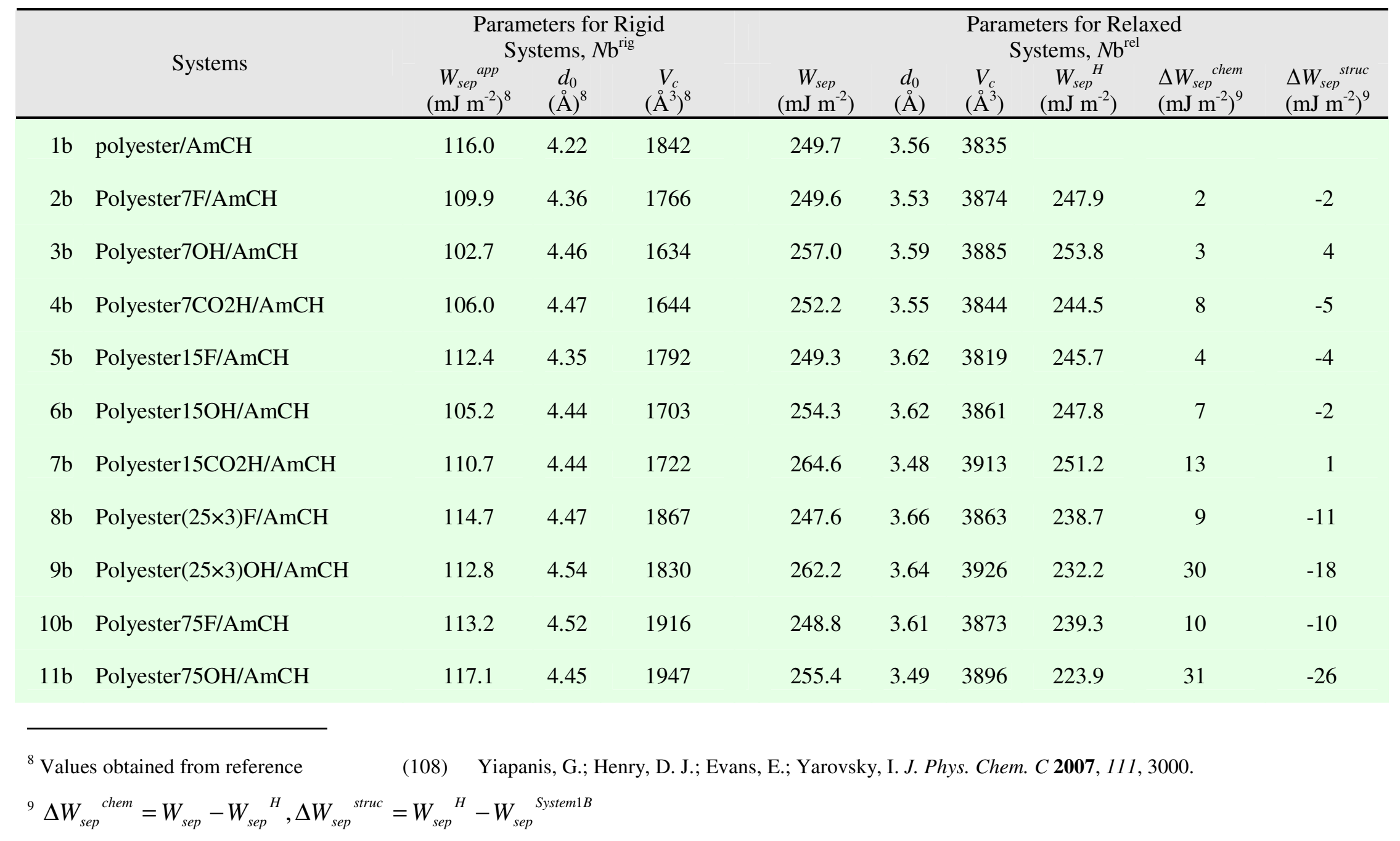


It can be seen from Table 5.2 that, following relaxation, modified polyesters generally exhibit slight increases in adhesion with amorphous carbon relative to the unmodified polyester. In particular, carboxylated and hydroxylated interfaces exhibit $\sim 1$ $6 \%$ increases in $W_{\text {sep }}$, while fluorinated systems exhibit no significant changes in $W_{\text {sep }}$ $(\sim 0-1 \%)$. In comparison, the previous chapter of this study showed that, for artificially rigid as well as locally relaxed polyester surfaces, the presence of substituents resulted in significant adhesive reductions with amorphous carbon ( 11-19\%). Therefore, there again appears to be a general convergence of the adhesion values toward those of the base interface (polyester/AmCH). This is further highlighted by the negligible variations in parameters $d_{0}(\sim 0.0-0.1 \AA)$ and $V_{c}(\sim 0-2 \%)$ of the modified systems relative to the unmodified interface.

Table 5.2 reveals that the work of separation values for the hydrogenated interfaces $\left(W_{\text {sep }}{ }^{H}\right)$ are again smaller than the corresponding functionalised values $\left(W_{\text {sep }}\right)$. Likewise, the chemical and structural components displayed in Table 5.2 exhibit trends very similar to those observed for graphite systems. Firstly, the chemical contribution values $\left(\Delta W_{\text {sep }}{ }^{\text {chem }}\right)$ for the Polyester $n \mathrm{X} / \mathrm{AmCH}$ interfaces lead to similar increases in interfacial adhesion ( 1-12\%). Secondly, in highly modified interfaces (systems 8b-11b), the effective residue-carbon interaction $\left(\Delta W_{\text {sep }}{ }^{\text {chem }}\right)$, results in a stronger attraction at the interface $(\sim 4-12 \%)$ compared to the equivalent interaction for low level modified interfaces $(\sim 1-5 \%)$. Finally, for each of these highly functionalised systems, the conformation of the polymer substrate leads to significant reductions in adhesion $\left(\Delta W_{\text {sep }}^{\text {struc }}\right)$ counteracting the chemical effects. These trends are consistent with those previously observed using the local relaxation procedure (see Section 4.4.2). However, the relaxed interfaces presented in Table 5.2 (Systems $n \mathrm{~b}^{\text {rel }}$ ) exhibit significantly reduced variations in $\Delta W_{\text {sep }}^{\text {struc }}$ compared to their energy minimised equivalent systems (Systems $n \mathrm{~b}^{\mathrm{min}}$, Table 5.2) indicating an overall convergence of adhesive behavior toward the base interface.

Despite the obvious similarity between the graphite and amorphous carbon systems, there are a number of subtle variations. Comparison of Table 5.2 and Table 5.1 reveals that the $W_{\text {sep }}$ and $V_{c}$ values for the amorphous carbon interfaces are all greater than those for the corresponding graphite interfaces. In conjunction with this, the equilibrium 
separation of the various polyester surfaces from the amorphous carbon model is smaller than that of the equivalent graphite interfaces. This variation in parameters is due primarily to the difference in the atomic structure of the systems (Figure 4.4) rather than the presence of partial atomic charges in the amorphous carbon model (see Section 4.4.1). The irregular surface structure of the amorphous carbon model, presents a larger surface area than graphite and due to interlocking, results in a larger interfacial contact and a smaller interfacial separation with the opposing polymer layer. This interlocking is

further evident from the fact that the $W_{\text {sep }}{ }^{H}$ values of the amorphous carbon interfaces are larger than those for the corresponding graphite equivalents.

Experimentally $^{2}$ it has been suggested that low gloss paints exhibit the most significant soil-derived color changes. The reason is attributed to the fact that valleys and pores on the surface of low-gloss paints entrap contaminants more easily that the smooth surface of high-gloss paints. Our findings in conjuction with this article suggest that sub-micron scale surface roughness can in fact increase interfacial adhesion, provided that the groove spacing (distance between protrusions) of one surface is sufficiently wide to cause interlocking with the opposing phase.

\subsubsection{Discussion}

The aging of the polymer surfaces has been simulated by undertaking molecular dynamics (MD), allowing the polyesters to relax at the interface with carbon. Following relaxation, we have noted a convergence of adhesion parameters for the polymer/graphite and polymer/amorphous carbon systems. Inagaki et al. ${ }^{154}$ observed that hydrophilically modified polyesters, aged at room temperature, converge to a similar value of contact angle (61-64 ) irrespective of the large difference in contact angle among the 'just modified' surfaces. While we have not determined the contact angle of our aged polymers, one might expect that due to the strong convergence of adhesion parameters, these polymer surfaces would exhibit similar values in contact angle.

The relaxation of the polymers in proximity of the hydrophobic carbonaceous surfaces also results in the migration of hydrophobic segments from the polymer bulk to the outer surface of the polyester and toward the carbon interface. To support this observation we have included snapshots of the polyester/graphite interface obtained 
under a rigid pre-aged regime (Figure 5.1a) and from the later stages of MD (Figure $5.1 b)$. It can be seen from these images that relaxation leads to the movement of phenyl rings toward the graphite interface.

(a) Rigid polyester/graphite interface

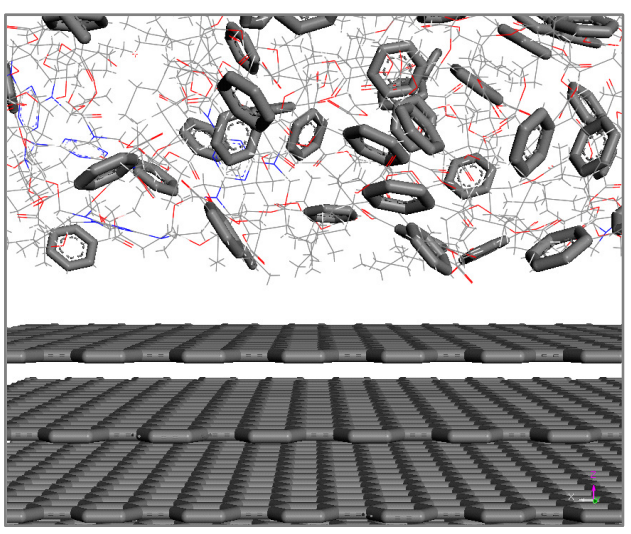

(b) Relaxed polyester/graphite interface

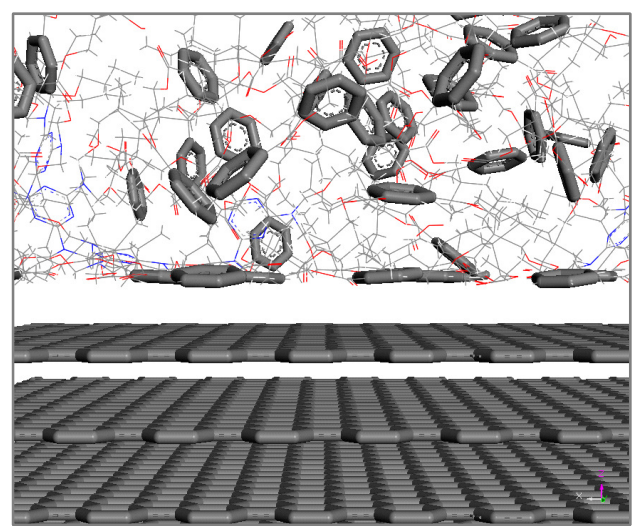

Figure 5.1 Snapshots of the polyester/graphite interface highlighting the repositioning of phenyl rings as a result of MD.

Figure 5.2a,b presents the concentration profiles of aromatic carbon atoms in selected polyester models, as a function of their average equilibrium separation from graphite and amorphous carbon surfaces, respectively. These plots have been obtained by averaging the concentration profiles of the relaxed interface systems during equilibrium stages of molecular dynamic simulations. Concentration profiles of aromatic carbon in the rigid base polyesters have also been included for comparison. It can be seen from both figures that, upon relaxation of the base interfaces (polyester/graphite or polyester/AmCH), the first peak shifted from $\sim 3.8$ to $\sim 3.4 \AA$. This shift is attributed to the migration of aromatic carbon atoms toward the interface region and to the alignment of these aromatic rings with respect to the graphitic layers. In fact, the separation between the polyester aromatic rings and the first graphitic layer of each carbon surface is $\sim 3.4 \AA$ and coincides with the interlayer spacing of the graphite and the average interlayer spacing of the amorphous carbon structures. Similarly, for the modified polyester models, relaxation also results in this carbon stacking arrangement at the interface. Such parallel stacking has been experimentally confirmed by atomic force microscopy (AFM) for a conjugate polymers and single-wall nanotube interfaces. ${ }^{184}$ Occhiello and co-workers, ${ }^{64}$ who used XPS to analyze the surface of plasma-treated 

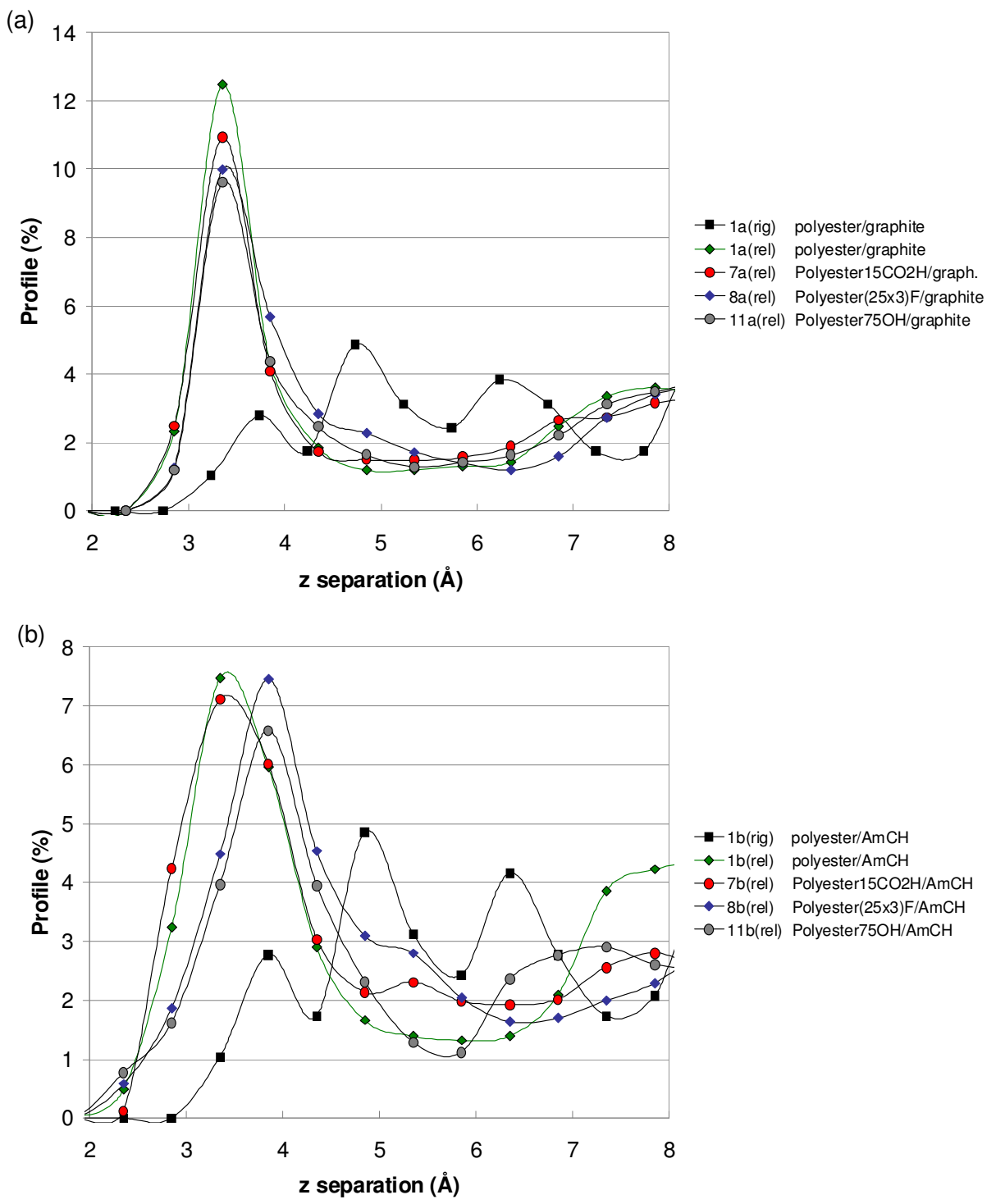

Figure 5.2 Concentration profile of carbon atoms in phenyl rings for various polyester models, as a function of the average separation from the graphite and amorphous carbon boundaries.

polystyrene (PS) samples, were able to show that the shakeup peak, associated with the aromatic ring of the repeating units, disappears directly after treatment. However, after low temperature aging in air, this peak becomes observable again indicating the reemergence of aromatic carbons near the polymer surface. Wen et al. ${ }^{41}$ investigated the stability of plasma fluorination of PET film by dipping the film into potassium hydroxide $(\mathrm{KOH})$ aqueous solution. After dipping, the treated PET films were characterized by XPS analysis, revealing that there was an increase in the carbon content 
at the surface of the film. These carbon atoms were partly associated with carbon types in phenyl rings, and despite aging in a hydrophilic medium, the strong affiliation between hydrophobic segments was clearly apparent.

Figure 5.2a,b also reveals that broader peaks are formed for the amorphous carbon interfaces, compared to the equivalent graphite interfaces. The broader peaks are attributed to the irregular (buckled) surface profile of the amorphous carbon model, which causes variations in the orientation of the aromatic rings in the polymer. In contrast, the graphite model comprises of an ideally smooth surface, and so the opposing rings in the polyester are arranged in similar parallel orientations.

To quantify variations in surface composition of the modified aged surfaces, a scale representation of the modified $\mathrm{Na}^{\text {rel }}$ and $\mathrm{Nb}^{\text {rel }}$ systems is presented in the following page, Figure 5.3. Illustrated graphically for each system is its $d_{0}$ value, together with the relative distance of its highest substitituent and its remaining subsituents from the carbon boundary surfaces (graphite and amorphous carbon).

First, the level of fine scale roughness exhibited by the various modified polyester models can be approximated by the position of the highest substituent, relative to the $d_{0}$ value of the interface (i.e., the separation of the highest modifier from the polyester surface boundary). For rigid interfaces, the highest substituents were located at a separation of $\sim 1.4-3.0 \AA$ from the polymer boundary. ${ }^{108,116}$ Figure 5.3 shows that following relaxation there is now a reduction in the separation of the highest substituent from the polyester surface. In particular, relaxation of the PolyesternX/graphite interfaces results in the highest substituents being located at a separation of $\sim 0.6-1.8 \AA$ from the polymer boundary. For the PolyesternX/AmCH interfaces, the separation of the highest substituent from the polyester is now $\sim 0.1-2.4 \AA$. Therefore, the protruding modifiers for all levels and types of functionalisation are now located closer to the surface boundary of the polymer, compared to the artificially rigid structures. This is a result of the overall migration of the polymer bulk toward the carbon surface in conjunction with the flattening of the "outer" layer (atomic smoothing). As a result, the physical effect of accentuated fine-scale roughness that helps reduce the effective contact area between the surfaces for artificially rigid interfaces is diminished by relaxation. 


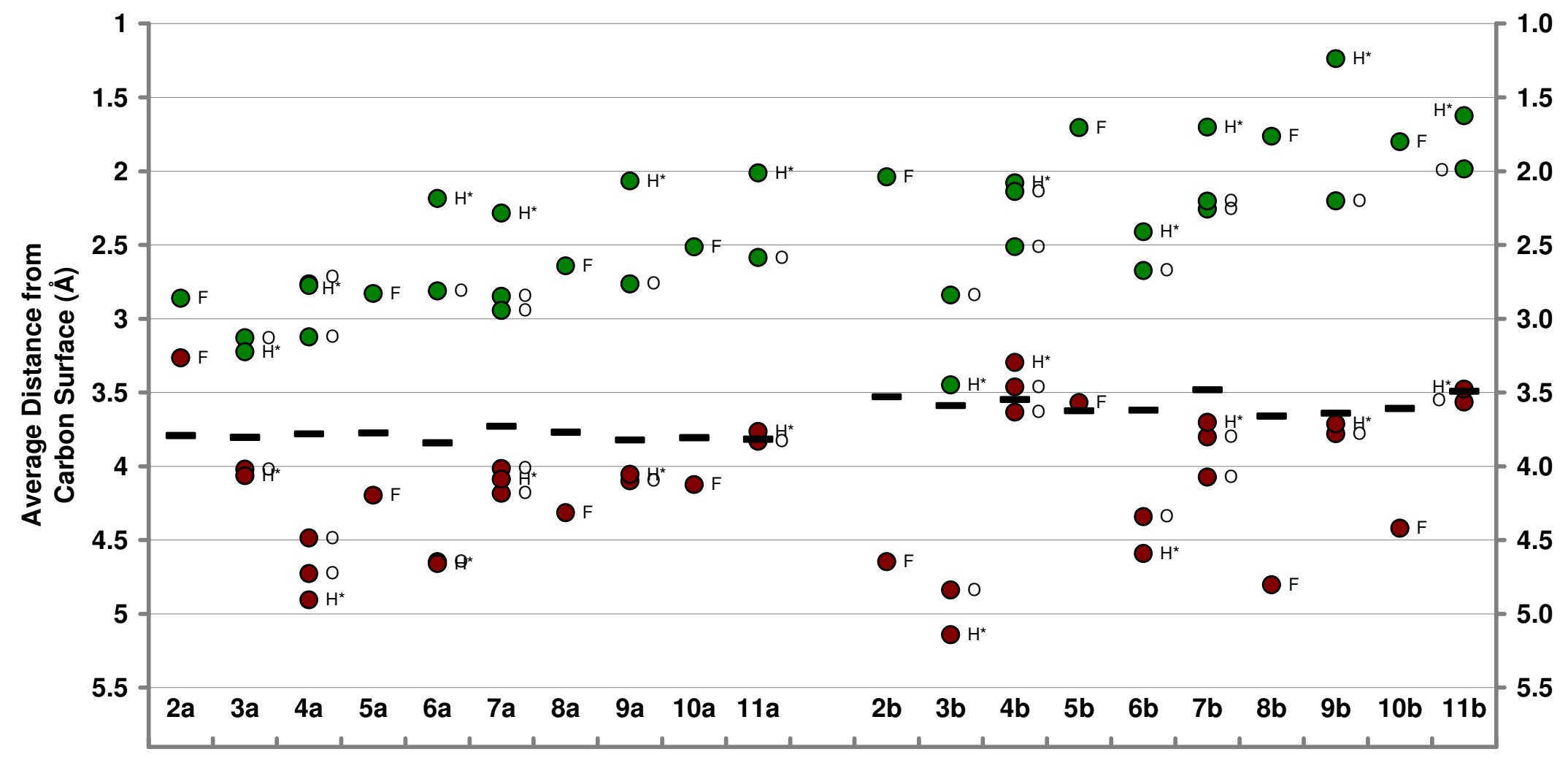

Relaxed Structures (Systems $\mathrm{Na}^{\text {rel }}$ and Systems $\mathrm{Nb}^{\text {rel }}$ )

-Equilibrium Separation

Figure 5.3 Scale representation of the average separation between carbon and polyester substituents, as well as the equilibrium separation for the relaxed interfaces; Polyester/graphite (Systems $\mathrm{Na}^{\mathrm{re}}$ ) and Polyester/AmCH (Systems $\mathrm{Nb}^{\mathrm{rel}}$ ). 
Second, in the relaxed interfacial configuration, the $\Delta W_{\text {sep }}^{\text {chem }}$ values indicate that the net residue-carbon interactions result in an increase in adhesion at the interface. Analysis of the force-field parameters reveals that the effective vdW interaction between aromatic carbon and substituent atom types $(\mathrm{C}, \mathrm{H}, \mathrm{O}$, and $\mathrm{F})$ is attractive, for interatomic separations larger than $\sim 3 \AA$ (see Figure 4.2, Section 4.4.1). It can be seen from the scale representation of Figure 5.3 that, for each aged system, the average separation of the substituents from the model carbon surfaces is $\sim 3.3-5.2 \AA$. Therefore, repulsive effects of polyester modification, which lead to reduction in adhesion to carbon under a rigid regime, are generally removed following relaxation of the interfaces. This is because the functional groups which are initially affixed at the surface of the polyester generally "turn away" from the interface and into the bulk of the polymer during relaxation of the systems.

The reorientation of surface modifiers away from the interface is due to a combination of various non-bond interactions that can occur at the polyester/carbon interface. The overall flattening of protruding modifiers can be related to the vdW attraction between substituents and the underlying polymer bulk. The flattening of protruding hydrogens in $\mathrm{OH}$ residues can also be related to formation of intra-molecular hydrogen bonds. ${ }^{108}$ Also, the carbon stacking arrangement may push hydrophilic modifiers further toward the bulk of the polymer.

Several aging studies have shown that treated polyesters tend to recover to their native state due to a surface reorganization that is very similar to what we have observed here. ${ }^{154,185}$ In these studies the aging behavior, accompanied by the movement of newly formed groups at the surface towards the bulk of the polymer, was attributed partly to the polymer surface instability and partly to minimisation of the surface free energy with the opposing phase. For example, Xanthopoulos and co-workers ${ }^{185}$ observed the hydrophobic recovery of plasma treated PET films, and found that newly formed polar groups at the surface move toward the bulk of the polymer. Moreover, the mobility of these polar segments was shown to increase with increasing storage temperature leading to accelerated aging. Wen at al. ${ }^{41}$ evaluated the stability of $\mathrm{CF}_{4}$ plasma-treated PET samples conditioned in a potassium hydroxide solution. They noted a smooth decrease in the F/C ratio and water contact angle of the surface of the film, which could be attributed 
to the reorientation of fluorine containing groups away from the top layer. Similarly, Selli et al. ${ }^{39}$ detected a lower surface content of fluorine 30 days after plasma treatment of PET surfaces conditioned under humid air. In contrast, Charpentier et al. ${ }^{149}$ found that UV/ozone-treated polyesters remained substantially stable, without any changes in surfaces properties for up to $480 \mathrm{~h}$ under ambient conditions.

We have also observed that the rearrangement of the polyesters and the stability of modifiers during relaxation may depend on the total number of functional groups initially affixed at the surface of the polyester (i.e., on the surface density of the modifiers). We note that, for highly modified systems, the structural effects on adhesion become apparent and lead to notable reductions in $\Delta W_{\text {sep }}^{\text {struc }}$, compared to the low level functionalised systems. It is believed that highly functionalised polyester surfaces exhibit less mobility than the Polyester7X or Polyester15X models during relaxation, largely due to the high density of residues $\left(4.7\right.$ residues $\left./ \mathrm{nm}^{2}\right)$ inhibiting polyester relaxation against the carbon models. Supporting this possibility is the observation in Figure 5.2a,b that, for the highly modified systems, the heights of aromatic carbon peaks are somewhat lower and, for the amorphous carbon interfaces, the peaks are displaced to higher separations in comparison to the base and low level modified systems. It can also be seen from Figure 5.3 that, for the Polyester $(25 \times 3) \mathrm{X}$ and Polyester75X systems, the separation of the highest substituent from the polymer boundary is marginally larger than that for the corresponding Poylester7X or Polyester15X systems, indicating that highly modified polymers exhibit more atomically rough surface profiles than low level modified polyesters, following relaxation.

Selli et al. ${ }^{39}$ reported on the stability of $\mathrm{SF}_{6}$ plasma-treated PET surfaces. They observed that the samples treated under relatively high radio frequency (rf) power exhibited a greater amount of fluorine content immediately after treatment compared to the low-frequency-treated samples. The high-frequency treated samples also exhibited a smaller decrease in the $\mathrm{F} / \mathrm{C}$ ratio during storage in humid air and maintained greater hydrorepellence properties than the less modified low rf treated samples.

Despite the increased stability observed for highly modified polymer surfaces, the attractive residue-carbon interaction $\left(\Delta W_{\text {sep }}{ }^{\text {chem }}\right)$ for these systems generally offsets any structural effects on adhesion, and overall, under this fully relaxed regime, 
functionalised polyester surfaces show negligible reductions in adhesion to carbon $(\sim 1$ 4\%). All modified polyester surfaces exhibit a convergence of interfacial adhesive behavior toward that of the unmodified interface, and this is a clear indication that the relaxation procedure used in this study can be related to the observed aging of modified polyester surfaces. As noted previously, this was not particularly evident when the energy minimisation technique was used to model relaxation of the surfaces. In fact, surface substituents were still shown to lead to notable reductions in adhesion with the graphite or amorphous carbon models (10-19\%). Results presented in this study show that extensive MD simulation under elevated temperature achieves significant restructuring of the polymer models, in qualitative agreement with experiment. ${ }^{185}$

\subsection{Conclusion}

Classical molecular dynamics was used to examine how aging of functionalised polyester surfaces effects interfacial adhesion between the polyester and carbon surfaces. All polyester surfaces undergo considerable changes in surface composition and morphology, leading to significantly increased adhesion to carbon compared to the initially rigid polymers. Under this fully relaxed regime, functionalised polyester surfaces exhibit negligible reductions in adhesion to carbon relative to the unmodified polyester and, in some cases, lead to slight increases in interfacial adhesion. This is because the functional groups, which are initially affixed at the surface of the polyester, generally "turn away" from the interface and into the bulk of the polymer during relaxation of the systems. Overall, the modified polyester surfaces exhibit a convergence of interfacial adhesive behavior toward that of the unmodified interface, and this is a clear indication that the relaxation procedure used in this study reflects the aging process of modified polyester surfaces. These results further indicate that for surface modification to be effective (i.e., reduce adhesion to carbon in the absence of water) the polyester surfaces need to be made more rigid prior to functionalisation. 


\section{Chapter 6}

\section{Polyester Surfaces and Fullerene in Aqueous Environment}

\subsection{Overview}

In this chapter, we will extend our molecular dynamics investigation of contaminant adhesion in vacuum to include an aqueous environment by modelling the interaction of fullerene $\mathrm{C}_{60}$, a soot-like contaminant particle, with polyester surfaces in water. We will firstly examine the effects of polymer surface modifications in the form of fluorine and hydroxyl functionalisation, on the hydrogen bonding network near the polymer. Subsequently we will investigate the influence of surface morphology, chemical modification and water-layer thickness separately on the nature of interaction between polyester and fullerene under an aqueous environment. 


\subsection{Introduction}

The underwater behaviour of polymer surfaces has attracted a great deal of interest in the field of anti-fouling coatings. Anti-fouling coatings are surfaces that are free from the accumulation of unwanted particulates or organisms in aqueous environments. One approach used to design such coatings, is to fabricate polymer surfaces that prefer to be in contact with water rather than the adherent particle. This can be achieved in theory by increasing the hydrogen bonding interactions of water with the surface. For example, Li et al. ${ }^{186}$ investigated the antibacterial behaviour of poly(ethylene terephthalate) (PET) surfaces modified by ion implantation with silver ions $\left(\mathrm{Ag}^{+}\right)$. They found that when silver was successfully implanted into the surface of the PET film there was a reduction in the water contact angle of the surface from 83.5 to $67.6^{\circ}$. Consequently, the quantity of Staphylococcus epidermis SE, an essentially hydrophobic bacterium, adhered onto the Ag-implanted PET film was less than $24 \%$ of the unmodified PET surface. The free energy of adhesion of contaminant on the PET surfaces was determined via Lifshitz-van der Waals/acid-base theory. ${ }^{187}$ For the Ag+ implanted PET film, the free energy of adhesion was found to be positive, indicating that SE adhesion is energetically unfavourable for this case. An alternative approach used to engineer anti-fouling coatings is to design immersible superhydrophobic surfaces. ${ }^{188,189}$ Attard ${ }^{189}$ showed that, when super-hydrophobic surfaces are immersed in water, air bubbles are trapped in micro- and nano-sized pores at the solid surface. Consequently, a mix of solid-liquid and solid-gas interfaces are created. The extent of solid-gas interface is proportional to the degree of hydrophobicity of the material. The higher the hydrophobicity, the larger the solid-gas interface will be, and the air bubble layer creates a barrier that may prevent adsorption of micro-organisms in the short term. Similarly, by maintaining an air film between the polymer surface and the water, the contaminant particle has a lower probability of encountering the surface.

In addition to the chemistry of the surfaces, several studies have shown that surface morphology plays a vital role in mechanical defence against fouling. ${ }^{190}$ Scardino et al. ${ }^{191}$ tested the attachment of various algae species on laser-etched polyimide surfaces in static laboratory bioassays, while Schumacher et al. ${ }^{192}$ studied the effect of surface 
morphology on the settlement of zoospores on poly(dimethylsiloxane) surfaces. These studies demonstrate that adhesion strength is related to the number of attachment points available to the marine organisms on the surface. Provided the primary length scale of roughness is smaller than the contaminant particle, then the expected fouling by organisms should be reduced with increasing roughness, as there are fewer attachment points. Zhang et al. ${ }^{68}$ investigated the fouling behaviour of smooth and nano-scale roughened super-hydrophobic coatings. The effect of nano-scale surface roughness on the adhesion of micro-foulants for periods of up to six months was assessed using visual and wettability measurements. Detailed analysis indicated virtually no organism attached to the super-hydrophobic surfaces in the first weeks of immersion, and the very rough surfaces exhibited high resistance to fouling over a six-month period. In comparison, smooth substrates exhibited fouling within a day.

In this chapter, we investigate the effects of surface modifications on the interaction of fullerene, a soot-like contaminant particle, with polyester in water. The purpose of this investigation is to identify design aspects which may be implemented onto polymer coatings to prevent adhesion of water-borne carbon based particulates. In Section 6.4.1 of this study, we perform molecular dynamics (MD) simulation of a film of water in contact with three polyester based surfaces: unmodified polyester, fluorinated polyester and hydroxylated polyester. Here, we examine the effects of polyester modification on the local organization of the hydrogen bonding network near the surface. In Sections 6.4.2 and 6.4.3, we undertake a series of comparative MD simulations of three hydrated surfaces interacting with fullerene. We investigate the effects of surface morphology, chemical modification, and water layer thickness separately on the nature of interaction between polyester and fullerene under an aqueous environment.

\subsection{Molecular Models and Theoretical Procedures}

\subsubsection{Composition of Molecular Models}

For this specific investigation, three polyester based surfaces were modelled: unmodified polyester (polyester), fluorinated polyester (Polyester75F), and hydroxylated polyester (Polyester75OH). Complete descriptions of the models can be found in Section 3.2. The topographical features of these models are not uniform, with the surface morphology, 
chemical composition and atomic density varying across the plane of all three models. It is also important to note that the functional groups for the modified polyesters (Polyester75F and Polyester75OH) are not distributed uniformly across the surface, and therefore there are some highly modified regions and some areas with low density of functional groups. These observations are illustrated in Figure 6.1, which shows a crosssectional slice taken along the boundary of the unmodified polyester model. We have subdivided this polymer surface into five regions denoted A, B, C, D, and E. Each region encompasses an area of $320 \AA^{2}$. The number of functional groups and surface coverage for each region is also displayed. We have also included the contoured vdW slice along the $x-y$ plane to highlight variations in atomic roughness exhibited by the polyester surface. An iso-value of zero along the slice (pink region) indicates that the slice intersects the $\mathrm{vdW}$ radii of the atoms along the boundary, whereas an iso-value greater than zero (yellow, green and blue regions) indicates that the slice is above the vdW radii of the surface atoms. Therefore, the yellow, green and blue shaded areas represent cavities of $2 \AA, 3 \AA$ and $4 \AA$ deep, respectively, along the surface of the polyester model.

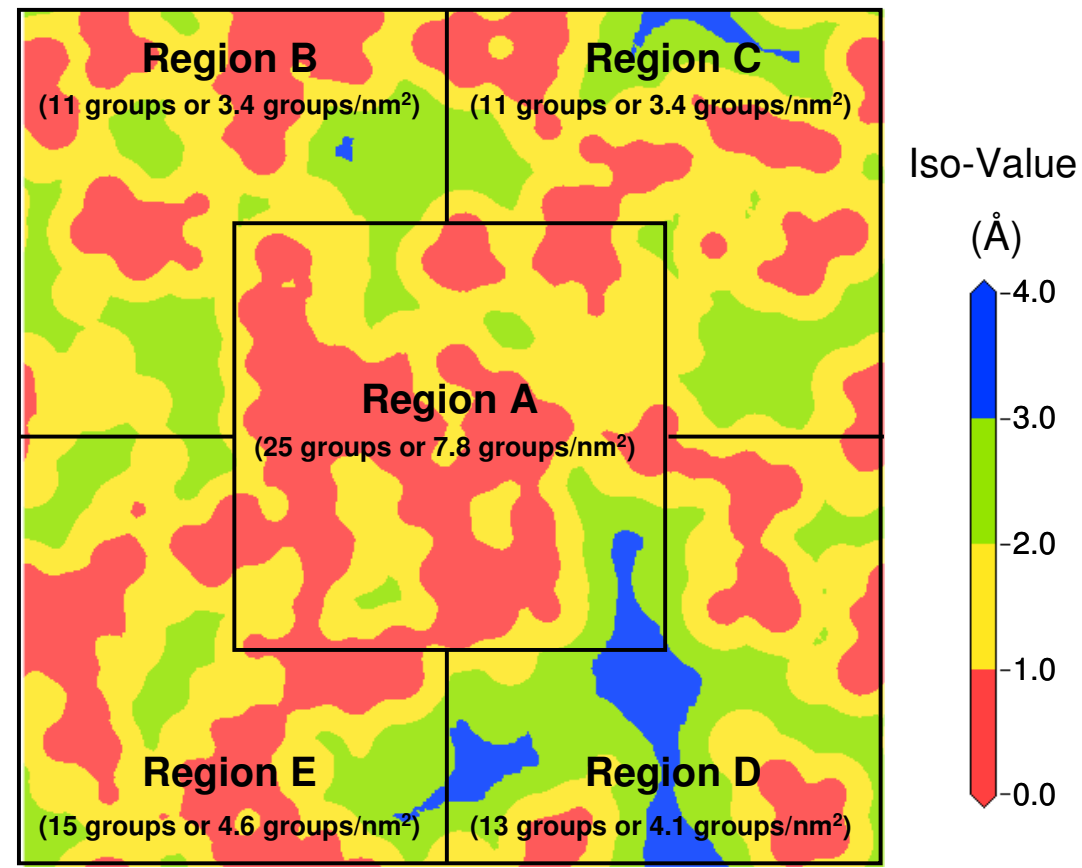

Figure 6.1 Contoured vdW slice of polyester model taken along the surface boundary in its initial configuration. 
Table 6.1 Characteristic Features of Specific Regions of the Polyester Surfaces

\begin{tabular}{|c|c|}
\hline region & major structural and chemical features \\
\hline A & $\begin{array}{ll}\text { - } & \text { high degree of surface roughness } \\
\text { - } & \text { non-porous } \\
\text { - } & \text { high subsurface density } \\
\text { - } & \text { moderate levels of crosslinking }\end{array}$ \\
\hline $\mathrm{B}$ & $\begin{array}{l}\text { - } \text { highly porous with deep as well as shallow grooves } \\
\text { - } \quad \text { high subsurface density } \\
\text { - } \quad \text { high concentration of aromatic carbons }\end{array}$ \\
\hline $\mathrm{C}$ & $\begin{array}{l}\text { - } \quad \text { highly porous with predominantly shallow grooves } \\
\text { - } \quad \text { high subsurface density } \\
\text { - } \quad \text { high levels of crosslinking }\end{array}$ \\
\hline $\mathrm{D}$ & $\begin{array}{l}\text { - characteristically large, deep cavity } \\
\text { - } \quad \text { low subsurface density }\end{array}$ \\
\hline $\mathrm{E}$ & $\begin{array}{l}\text { - } \quad \text { characteristically large, shallow cavity } \\
\text { - } \text { high subsurface density }\end{array}$ \\
\hline
\end{tabular}

Observation of Figure 6.1 reveals that the structure and morphology of the polymer surface are not uniform across all five regions with different levels of atomic surface roughness, crosslinking, subsurface density, and depressions or cavities. Presented in Table 6.1 is a summary of the major characteristics of each region corresponding to Figure 6.1. It is important to note here, that that the features of crosslinking presented in the table above, are associated with the original cured polymer surface model ${ }^{94}$ i.e., no 'deliberate' crosslinking has been undertaken. The crosslinks are concentrated in subsurface regions of the polymer film. In the next chapter (Section 7) there will be a shift in focus towards imparting rigidity to the outer-surface of the polymer through additional surface crosslinking.

In this section, polyester and water interfaces were constructed by solvating each of the polyester models with a $15 \AA$ thick water layer of density of $1 \mathrm{~g} / \mathrm{cm}^{3}$, above the surface boundary of the polymer. Each system was enclosed in a unit cell with 
dimensions $40 \AA \times 40 \AA \times 100 \AA$. Extension of the cell in the $\mathrm{z}$ direction ensures that adjacent systems do not interact along the z-axis when periodic boundary conditions are applied. As a result, infinite quasi-2D solvated interfaces were simulated.

The carbonaceous contaminant particle was modelled using a [60] fullerene particle. Refer to Section 3.3 for a complete description of this particular model. To simulate polyester surfaces interacting with contaminants under an aqueous environment, each polymer surface was combined with $\mathrm{C}_{60}$, and the resulting system, was solvated as described above. A schematic illustration of the polyester $/ \mathrm{H}_{2} \mathrm{O} / \mathrm{C}_{60}$ system is depicted in Figure 6.2. For these solvated polymer/carbon systems, the interfacial separation $(d)$ was defined as the distance between the polyester boundary and centre of mass of the fullerene particle. Several configurations were examined, with the fullerene particle initially placed at various locations ( $\mathrm{x}, \mathrm{y}$, and $\mathrm{z}$ coordinates) relative to the polyester surfaces. These configurations are detailed in the following section.

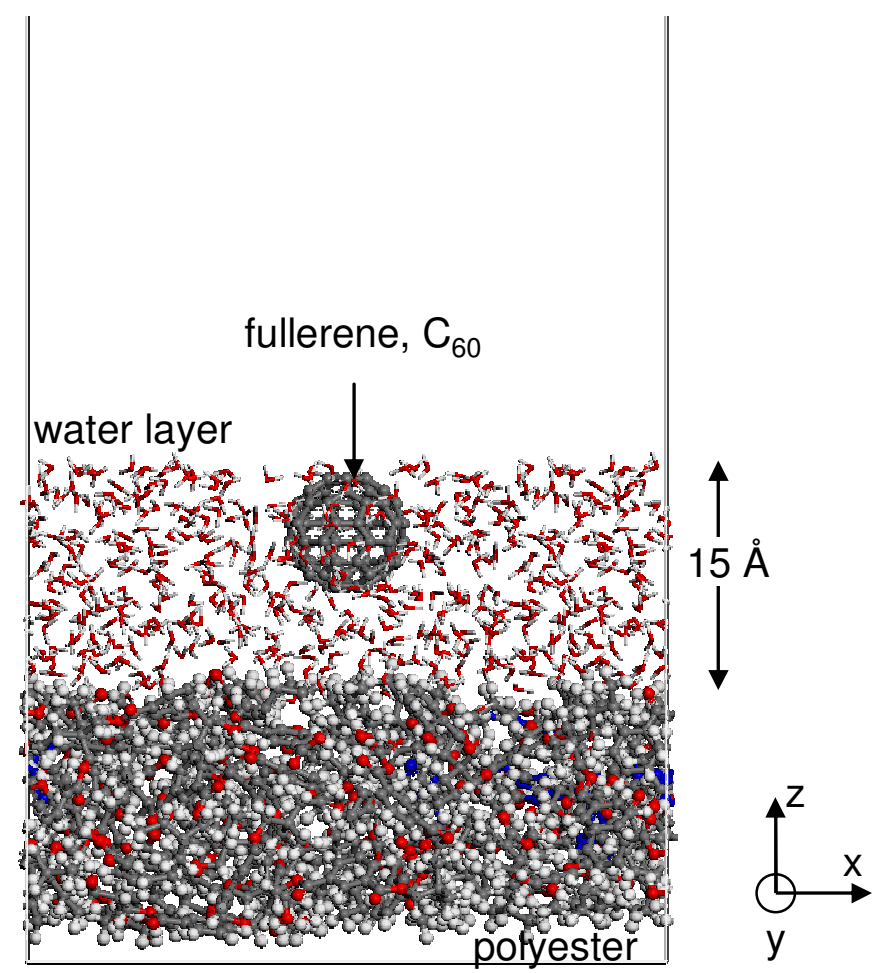

Figure 6.2 Schematic representation of the $\mathrm{C}_{60}$ particle interacting with a hydrated polyester surface, polyester $/ \mathrm{H}_{2} \mathrm{O} / \mathrm{C}_{60}$. The carbon nano-particle represents a soot particulate and the hydrated polymer film represents a wetted surface coating. 


\subsubsection{Theoretical Procedures}

For these hydrated systems, the simple point charge (SPC) method was used to model the water molecules. ${ }^{73}$ In this particular three site model, each atom gets assigned a point charge. Each hydrogen atom is assigned a charge of 0.41 e and the oxygen atom is assigned a charge of -0.82 e. The water model implemented within COMPASS however, is unlike the standard SPC model. ${ }^{193}$ One point of difference is that in COMPASS, the constituent atoms of water are treated as beads on a spring rather than rigid point charges. Prior to generation of a molecular dynamics (MD) trajectory, all systems were subjected to energy minimisation using the conjugate gradient procedure with a convergence criterion of $0.04 \mathrm{~kJ} \mathrm{~mol}^{-1}$. During energy minimisation, the carbon particle and polyester surfaces were kept fixed in their original geometry while the water molecules were free to move. Relaxation of the minimised systems was then undertaken by performing unconstrained MD simulations in the NVT ensemble for 2 ns, with the temperature maintained at $298 \mathrm{~K}$, using the Andersen thermostat, ${ }^{131}$ and a collision ratio of 1.0. Equilibrium was established after $1 \mathrm{~ns}$. Data were acquired for the final $1 \mathrm{~ns}$ of each molecular dynamics simulation run, using a 1.0 fs time step. To retain computational tractability, the atom-based non-bond summation method was used for MD simulations with a cut-off distance of $15.5 \AA$, a spline width of $5.00 \AA$, a buffer of $2.00 \AA$, and a long-range tail correction.

For the polyester/water systems, the local organization of the hydrogen bonding network was described by calculating values of the total number of hydrogen bonds $(\mathrm{H}-$ bonds) per water molecule, the number of H-bonds donated to and/or accepted from surface functional groups, and the average length of hydrogen bonds within the first hydration shell of polyester. The hydration shell was defined as the water molecules within $3 \AA$ from the nearest surface atom. These parameters were calculated as the average over the final 500 ps of the equilibrium trajectory. A hydrogen bond criterion was defined by a cut-off distance of $2.5 \AA$ and a donor-hydrogen-acceptor cut-off angle of $90^{\circ} .{ }^{194}$ Specific interactions of surface atoms with water and fullerene were characterized by averaging the radial distribution function $g(r)$ during the final $1 \mathrm{~ns}$ of trajectory. Values of $g(r)$ were normalized to account for the vacuum spacer introduced in the cell to mimic a 2D interface. 
Several systems were examined, and the MD simulations were started with the centre of mass of the $\mathrm{C}_{60}$ located at 6,8 , and $10 \AA$ from the polyester surfaces. At each of these interfacial separations, five separate MD simulations were run with the fullerene particle positioned in five different regions relative to the polyester surfaces (regions A-E, Figure 6.1). The interaction of fullerene with polyester in solution was evaluated by observing the displacement of the fullerene particle during simulation, as well as determining the average interfacial separation between fullerene and polyester once the equilibrium had been established. To determine the effect of water layer thickness on adhesion between $\mathrm{C}_{60}$ and polyester, we undertook several simulations using a $30 \AA$ thick water layer.
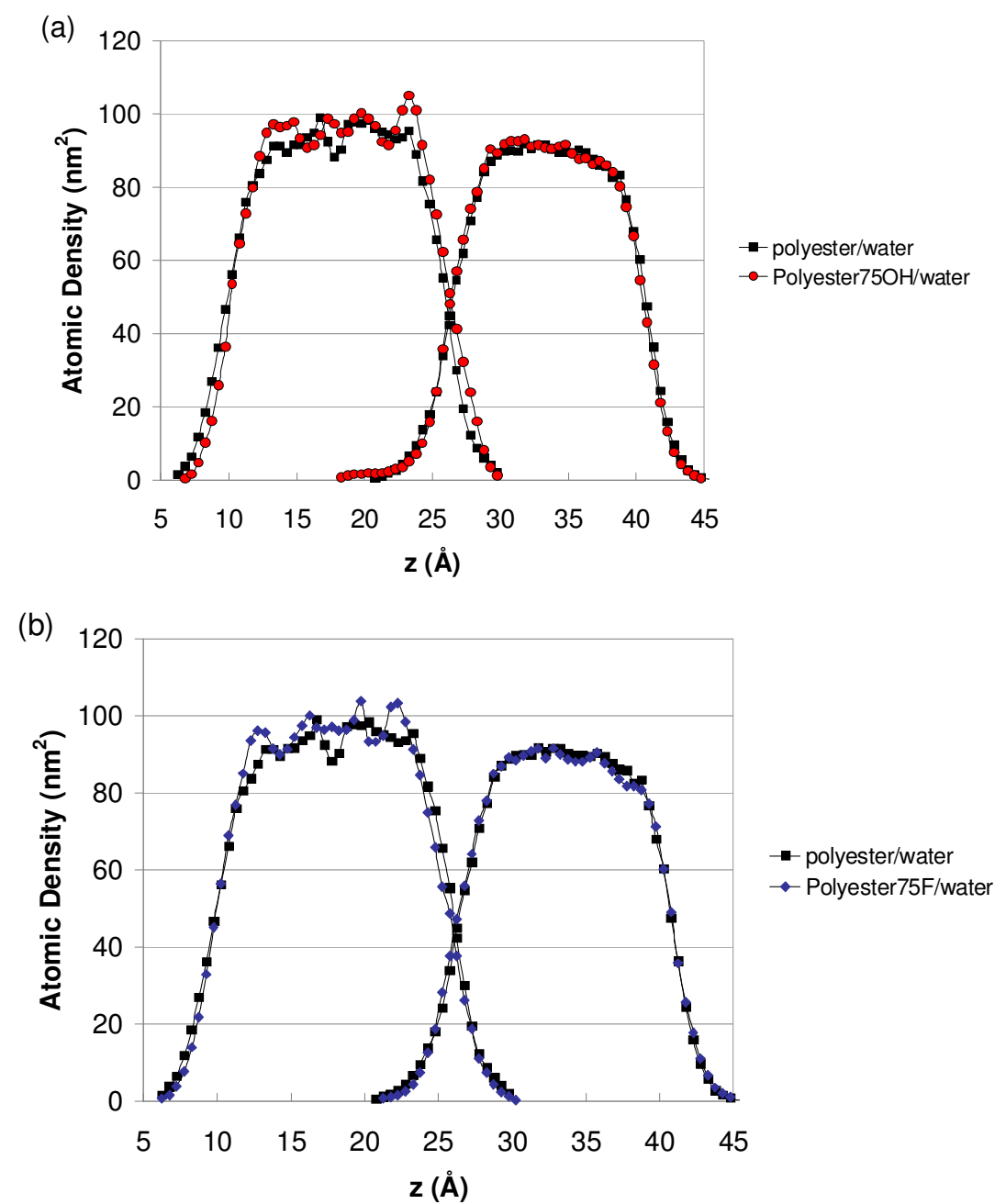

Figure 6.3 Concentration profiles of polyester $/ \mathrm{H}_{2} \mathrm{O}$ systems. Profiles of the polymer surfaces are displayed to the left-hand side of the graphs, while the water profiles are displayed toward the right-hand side. 


\subsection{Results and Discussion}

\subsubsection{The Polyester and Water Interface}

To determine the influence of the surface functionalisation on the water density, concentration profiles of the polyester/water systems were generated. These are presented in Figure 6.3.

All profiles were acquired by averaging the number of atoms in strips, parallel to the $\mathrm{x}-\mathrm{y}$ plane, of $0.5 \AA$ in thickness during the final $1 \mathrm{~ns}$ of molecular dynamics. We included the profiles of the individual components, polymer and water, that comprise each system as a function of $\mathrm{z}$ position. The density profiles of the hydroxylated $\left(\right.$ Polyester $\left.75 \mathrm{OH} / \mathrm{H}_{2} \mathrm{O}\right)$ and the fluorinated systems (Polyester $75 \mathrm{~F} / \mathrm{H}_{2} \mathrm{O}$ ) are displayed in Figure 6.3a and Figure 6.3b, respectively, while the untreated system $\left(\right.$ Polyester $\left./ \mathrm{H}_{2} \mathrm{O}\right)$ is included in both diagrams for comparison. The surface/water interface region of each system is arbitrarily defined as the region of coexistence of the two components (polymer and water) and is approximately located between 23 and $30 \AA$ along the $\mathrm{z}$ axis. Figure 6.3a shows that for the hydroxylated system, there is a slight increase in the concentration of atoms at the interphase region compared to the unmodified system. It can also be seen that the water molecules penetrate further into the "bulk" of the hydroxylated polyester in comparison to the unmodified substrate. Furthermore, the water profile for the hydroxylated interface is displaced slightly closer toward the polymer curve than that for the unmodified system, indicating a possible reduction in the water layer thickness. Interestingly, the concentration profile for the fluorinated interface (Figure 6.3b) exhibits no significant variation relative to that of the unmodified model.

Specific interactions between surface atoms and water are described using radial distribution functions $\mathrm{g}_{\mathrm{X}^{\prime}-\mathrm{O}}(\mathrm{r})$ and $\mathrm{g}_{\mathrm{X}^{\prime}-\mathrm{H}}(\mathrm{r})$ (presented in Figure 6.4, in the following page), where the first subscript $X^{\prime}$, represents the surface atoms of the polyester models, and the second subscript, $\mathrm{O}$ or $\mathrm{H}$, represents the oxygen or hydrogen atoms of the water molecules, respectively. In the case of the unmodified interface, Figure 6.4a, radial distribution functions (RDFs) of surface hydrogen atoms with water oxygen and hydrogen, $\mathrm{g}_{\mathrm{H}^{\prime}-\mathrm{O}}(\mathrm{r})$ and $\mathrm{g}_{\mathrm{H}^{\prime}-\mathrm{H}}(\mathrm{r})$, respectively, do not exhibit strong correlations, 
indicating that these interactions are not significant. Conversely, the radial distribution function of surface carbon atoms with oxygen atoms of water, $\mathrm{g}_{\mathrm{C}^{\prime}-\mathrm{O}}(\mathrm{r})$, exhibits a primary peak at $4.05 \AA$. Analysis of the COMPASS force-field parameters (see Figure 4.2) reveals that the vdW equilibrium separation between the carbon atom types of the polyester and oxygen atoms of water coincides with the position of this peak, reflecting the dominance of $\mathrm{vdW}$ forces for these pairwise interactions.
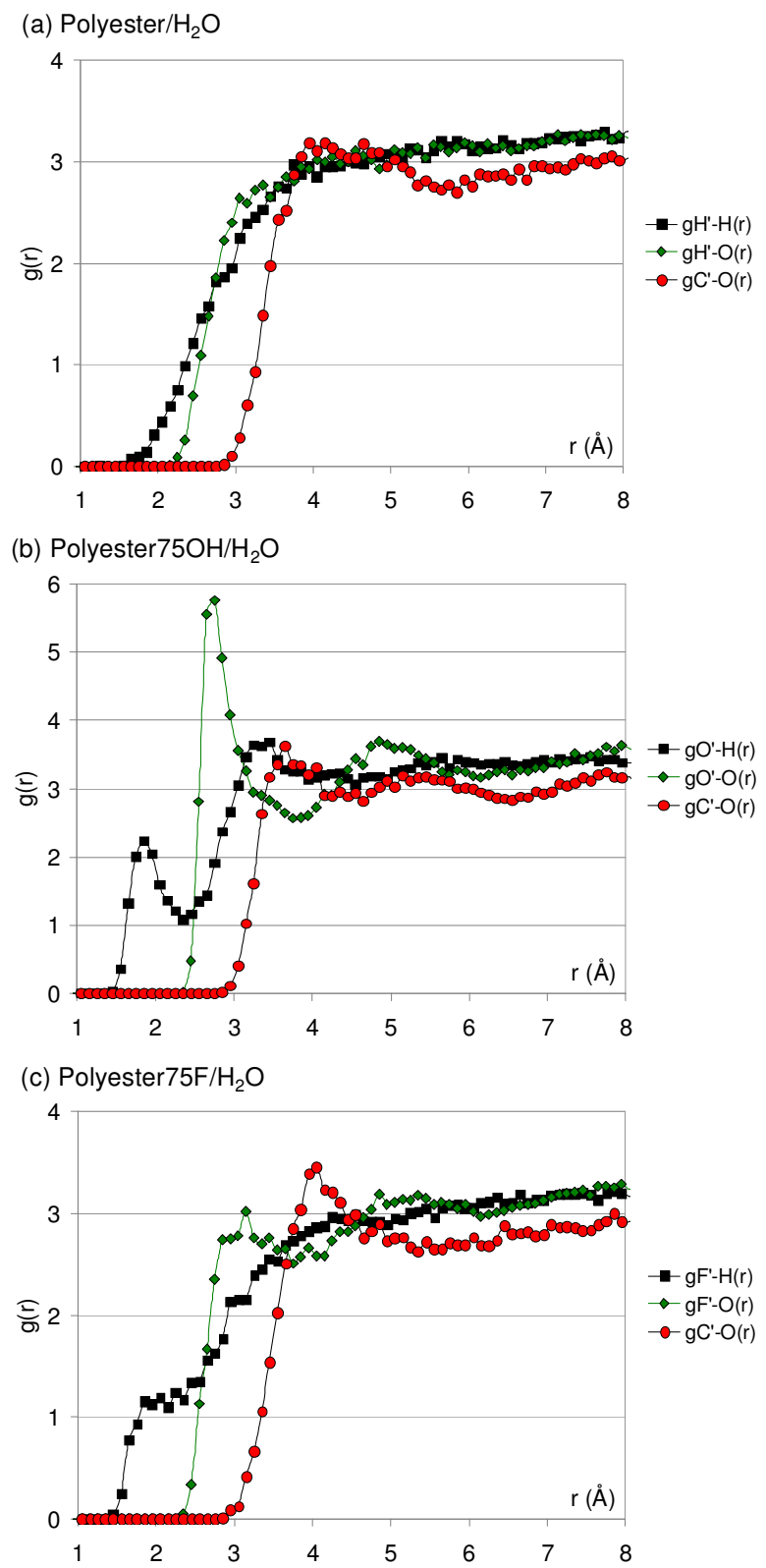

Figure 6.4 Radial distribution functions $g(r)$ of surface atoms in polyester (indicated by a prime), with $\mathrm{O}$ and $\mathrm{H}$ atoms of water. Values of $\mathrm{g}(\mathrm{r})$ are normalized to the overall density (including vacuum space) of the unit cell. 
So how do our nanoscale surface modifications in the form of hydroxyl or fluorine functionalisation effect the distribution of surrounding water molecules? Hydroxylation of the polyester was undertaken by replacing protruding hydrogen atoms with hydroxyl $(\mathrm{OH})$ substituents. Radial distribution functions of these hydroxyl groups with component atoms of water are presented in Figure 6.4b. The RDF of hydroxyl oxygen with hydrogen of water, $\mathrm{g}_{\mathrm{O}^{\prime}-\mathrm{H}}(\mathrm{r})$, exhibits a peak at $1.85 \AA$. The RDF between hydroxyl oxygen and oxygen of water, $\mathrm{g}_{\mathrm{O}^{\prime}-\mathrm{O}}(\mathrm{r})$, exhibits a peak at $2.75 \AA$. These peaks highlight that there is hydrogen bonding between water and hydroxyl groups of the surface. Moreover, the $\mathrm{g}_{\mathrm{C}^{\prime}-\mathrm{O}}(\mathrm{r})$ for the hydroxylated system exhibits an increase in magnitude and a shift to shorter separation $(\Delta \mathrm{r} \sim 0.5 \AA)$ compared to the $\mathrm{g}_{\mathrm{C}^{\prime}-\mathrm{O}}(\mathrm{r})$ for the unmodified and fluorinated systems, indicating a possible reduction in the spacing between the water and substrate.

In the case of the fluorinated polyester, replacement of "light" hydrogen atoms for "heavier" fluorine atoms is expected to result in a stronger vdW interaction with water; both a stronger short-range repulsion as well as a stronger long-range attraction (see Figure 4.2). However, there is also an increase in the strength of electrostatic interactions as a result of the average partial charge assigned to fluorine atoms, $-0.24 \mathrm{e}$, compared to that of the replaced $\mathrm{H}$ atoms of the unmodified surface, $+0.06 \mathrm{e}$. From Figure 6.4c, the radial distribution function $\mathrm{g}_{\mathrm{F}^{\prime}-\mathrm{H}}(\mathrm{r})$ exhibits a significant increase in intensity at short separations (less than $2.5 \AA$ ) compared with the $\mathrm{g}_{\mathrm{H}^{\prime}-\mathrm{H}}(\mathrm{r})$ of the unmodified interface in Figure 6.4a. This particular variation is indicative of the stronger attractive electrostatic forces between fluorine atoms and hydrogen atoms of water. The RDF of surface fluorine atoms with the oxygen atoms of water $\left(\mathrm{g}_{\mathrm{F}^{\prime}-\mathrm{O}}(\mathrm{r})\right)$ exhibits a slight increase in intensity and a marginal shift $(\Delta \mathrm{r} \sim 0.1 \AA)$ to larger separations compared to the $\mathrm{g}_{\mathrm{H}^{\prime}-\mathrm{O}}(\mathrm{r})$ of the unmodified interface. The $\mathrm{g}_{\mathrm{C}^{\prime}-\mathrm{O}}(\mathrm{r})$ for the Polyester $75 \mathrm{~F} / \mathrm{H}_{2} \mathrm{O}$ system also shows an increase in peak intensity as well as a minor shift to larger separations relative to its unmodified counterpart. 


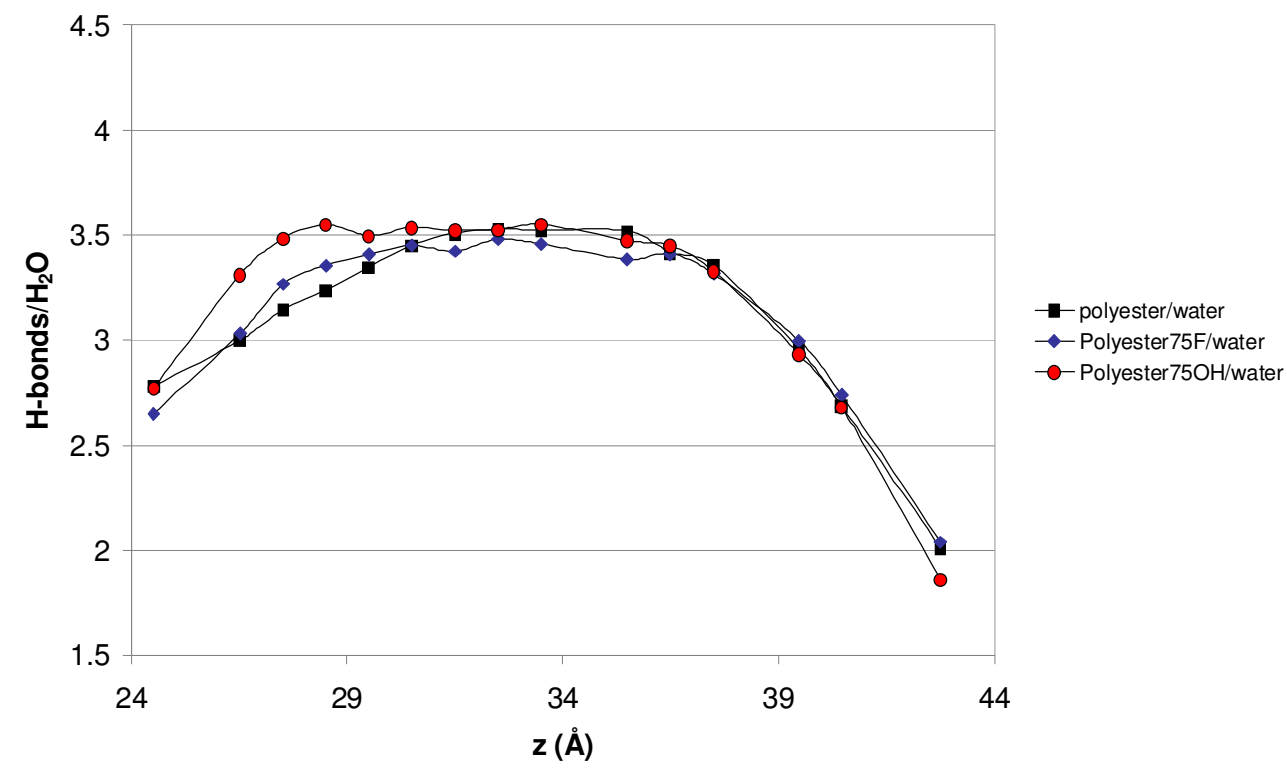

Figure 6.5 Variation in the total number of hydrogen bonds per water molecule with average $z$ position. Cut-off hydrogen bond distance of $2.5 \AA$ and cut-off hydrogen bond angle of $90^{\circ}$ were used as a criteria for $\mathrm{H}$-bond calculation. $^{19}$

To observe any disruption in the hydrogen bonding network of water caused by surface modification, we present the total number of hydrogen bonds formed per water molecule (H-bonds $/ \mathrm{H}_{2} \mathrm{O}$ ) as a function of $\mathrm{z}$ position (Figure 6.5). We previously noted that the surface/water interface is approximately located between 23 and $30 \AA$ along the $\mathrm{z}$ axis. The surface/vacuum interface is approximately located between 39 and $44 \AA$ in the $\mathrm{z}$ direction. This figure clearly shows a reduction in the number of hydrogen bonds per water molecule near the surface/water and surface/vacuum interfaces in relation to the middle region of the water layer (located between 30 and $39 \AA$ ). In particular, the average number of hydrogen bonds per water molecule changes from approximately 2.6 in the near surface region, to 3.5 in the centre of the water layer which is approximately the value for bulk SPC water. ${ }^{195}$ Near the polymer/water interface, there is a reduction in the number of hydrogen bonds per water molecule relative to the "bulk" region due to the reduced number of hydrogen bonding sites on the polymer surface. However, in the case of hydroxyl-modified polyester, each surface $\mathrm{OH}$ group can provide both donor and acceptor sites for hydrogen bonds, enabling a more complete hydrogen bonding network, compared to Polyester or Polyester75F. Near the polymer/water interface, fluorine 
modified polyester system exhibits a reduced number of hydrogen bonds per water molecule compared to its hydroxyl modified counterpart, but on average a greater number of hydrogen bonds per water molecule relative to the untreated surface.

To further clarify these results, we examined the hydrogen bonding network between surface atoms and water molecules located in the first hydration shell. This was arbitrarily defined as the layer of water molecules situated within a radial distance of $3 \AA$ from the nearest polymer atom. For the unmodified system, $25 \%$ of $\mathrm{H}_{2} \mathrm{O}$ molecules within the first hydration layer form a hydrogen bond with the surface with an average H-bond length of $2.05 \AA$. In comparison, water molecules near the hydroxylated and fluorinated polyester layers exhibit a significant increase in the number of hydrogen bonds with the substrate. In particular, near the Polyester $75 \mathrm{OH}$ surface, $100 \%$ of water molecules form a hydrogen bond with the surface, typically with a terminal $\mathrm{OH}$ group. The average length of these H-bonds is $1.99 \AA$ A. For the fluorinated system, $50 \%$ of water molecules form a hydrogen bond with surface atoms, typically with a fluorine atom, with an average bond length of $2.09 \AA$. Therefore, in conjunction with the observed trends in radial distribution functions, these results indicate that modification of the polyester surface with $\mathrm{OH}$ and $\mathrm{F}$ substituents, leads to stronger interactions between surface atoms and water. The strongest interaction between water and the surfaces is clearly exhibited by the hydroxyl modified polyester, while the fluorinated system exhibits more subtle changes relative to the untreated model.

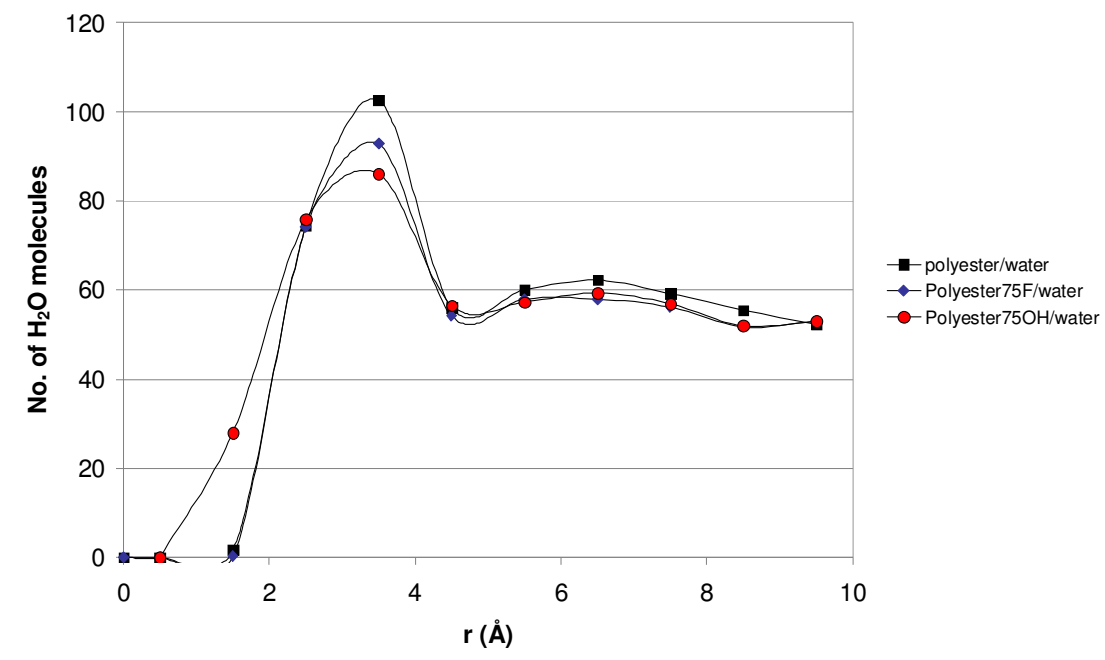

Figure 6.6 Number of water molecules as a function of radial distance from the nearest polymer atom. 
Variations in the spacing between surface and water were attained by profiling the number of water molecules as a function of separation from the nearest surface atom (Figure 6.6, previous page). Results show that hydroxyl modification leads to a reduction in the spacing between water and substrate compared to the untreated and fluorinated system, highlighting an increase in hydrophilic characteristics of this surface. This result is in qualitative agreement with the study of Janecek and Netz, ${ }^{196}$ who employed Monte Carlo simulations to study various wetting properties of hydroxylated hydrocarbonbased surfaces. In their study, water layers of approximately 40-50 ̊ thick were placed on the modified and unmodified surfaces and described by the standard SPC/E model at room temperature $(298 \mathrm{~K})$. Results show that, for hydroxylated surfaces, the strong affinity of water to hydroxyl groups leads to a significant lowering of the depletion thickness which is a measure of the distance between bulk phases. For the fluorinemodified system presented here, there is no significant change in the spacing between water and substrate compared to that of its unmodified counterpart (Figure 6.6). Both fluorinated and untreated systems maintain an "air" gap of $2 \AA$ between surface and water.

\section{(a) Polyester $75 \mathrm{OH} / \mathrm{H}_{2} \mathrm{O}$}

(b) Polyester75F/ $\mathrm{H}_{2} \mathrm{O}$

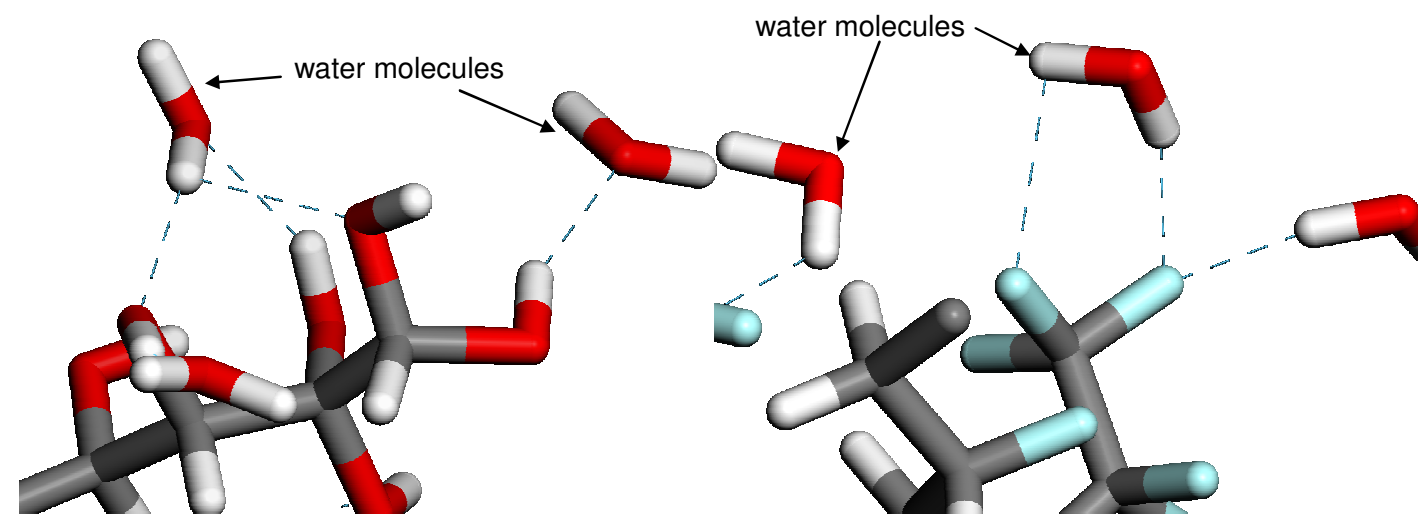

Figure 6.7 Snaphots of polyester/water systems highlighting hydrogen bonding configurations. For an improve clarity of the image, selected atoms have been omitted.

Displayed in Figure 6.7 are snapshot images of the polyester/water systems that highlight local structuring of water molecules typical for areas of high concentration of functional groups such as region A. For hydroxyl-modified polyester, each surface $\mathrm{OH}$ group can provide both donor and acceptor sites for hydrogen bonds. For this reason, 
water molecules at the hydroxyl-substituted interface Figure 6.7a) are typically oriented with one $\mathrm{H}-\mathrm{O}$ bond parallel to the surface plane enabling a more complete H-bonding interaction with the surface $\mathrm{OH}$ groups, while the other $\mathrm{H}-\mathrm{O}$ bond points away from the surface plane (x-y plane). Such a configuration of water molecules accounts for the increase in water density near its surface compared to Polyester or Polyester75F. This interaction also increases the degree of water penetrating the surface boundary as shown in Figure 6.3a. In contrast, the negatively charged fluorine attracts only the hydrogen atoms of water, often resulting in the $\mathrm{H}-\mathrm{O}$ bond of water pointing toward the surface plane and the other $\mathrm{H}-\mathrm{O}$ bond orientated parallel to and further from the surface plane (Figure 6.7b). This arrangement accounts for the stronger correlation observed in the RDFs between surface atoms and water for fluorine-modified polyester, compared to that of its unmodified counterpart. It also explains why there is no significant change in spacing between surface and water for the fluorinated system compared to that of its unmodified counterpart.

Table 6.2 Average Equilibrium Separation Values $\left(d_{a v}\right)$ between $\mathrm{C}_{60}$ and Hydrated Polyester surfaces ${ }^{x}$

\begin{tabular}{lccccc}
\hline \multicolumn{1}{r}{ system } & \multicolumn{5}{c}{ regions } \\
& A & B & C & D & E \\
\hline polyester $/ \mathrm{H}_{2} \mathrm{O}$ & 3.57 & -0.11 & 2.91 & 0.99 & 3.39 \\
Polyester $75 \mathrm{~F} / \mathrm{H}_{2} \mathrm{O}^{\text {xi }}$ & 4.51 & 4.18 & 3.63 & 2.35 & 3.92 \\
Polyester $75 \mathrm{OH} / \mathrm{H}_{2} \mathrm{O}^{\mathrm{ii}}$ & 5.15 & 4.14 & 4.85 & 1.77 & 4.85
\end{tabular}

\subsubsection{Adhesion of $\mathrm{C}_{60}$ on Hydrated Unmodified Polyester}

Presented in Table 6.2, are the average equilibrium separation values between fullerene $\mathrm{C}_{60}$ and the hydrated polymer surfaces. In the absence of water, van der Waals forces have been shown to be sufficiently strong to cause physisorption of the fullerene particle

\footnotetext{
${ }^{\mathrm{x}}$ For systems with initial separation of 6-8 $\AA$.

${ }^{\mathrm{xi}}$ Systems where fullerene was repelled from the surface are excluded in calculation of the average.
} 
onto polyester. ${ }^{98}$ Fullerenes are able to at least partially infiltrate the flexible polymer surface by opening surface cavities. In this section, we investigate how a water layer affects the interaction of the fullerene particle with our selected polyester surfaces. Interestingly, we find that, when molecular dynamic (MD) simulations are begun with fullerene positioned at $\sim 6-8 \AA$ from the unmodified polyester, the carbon particle is seen in all cases to be attracted toward the polymer substrate as was observed for the in vacuo system. Consequently, values of the equilibrium separation between $\mathrm{C}_{60}$ and unmodified polyester, which are displayed in Table 6.2 are less than the initial separation of 6 or 8 $\AA$. In a typical simulation, the hydrophobic carbon particle moves through the water layer, approaches the surface, and then establishes a close contact with the hydrophobic polyester. Presented in Figure 6.8 is an example of a typical approach curve for a polyester $/ \mathrm{H}_{2} \mathrm{O} / \mathrm{C}_{60}$ system, which also includes snapshots of the system in its initial and equilibrium configurations.

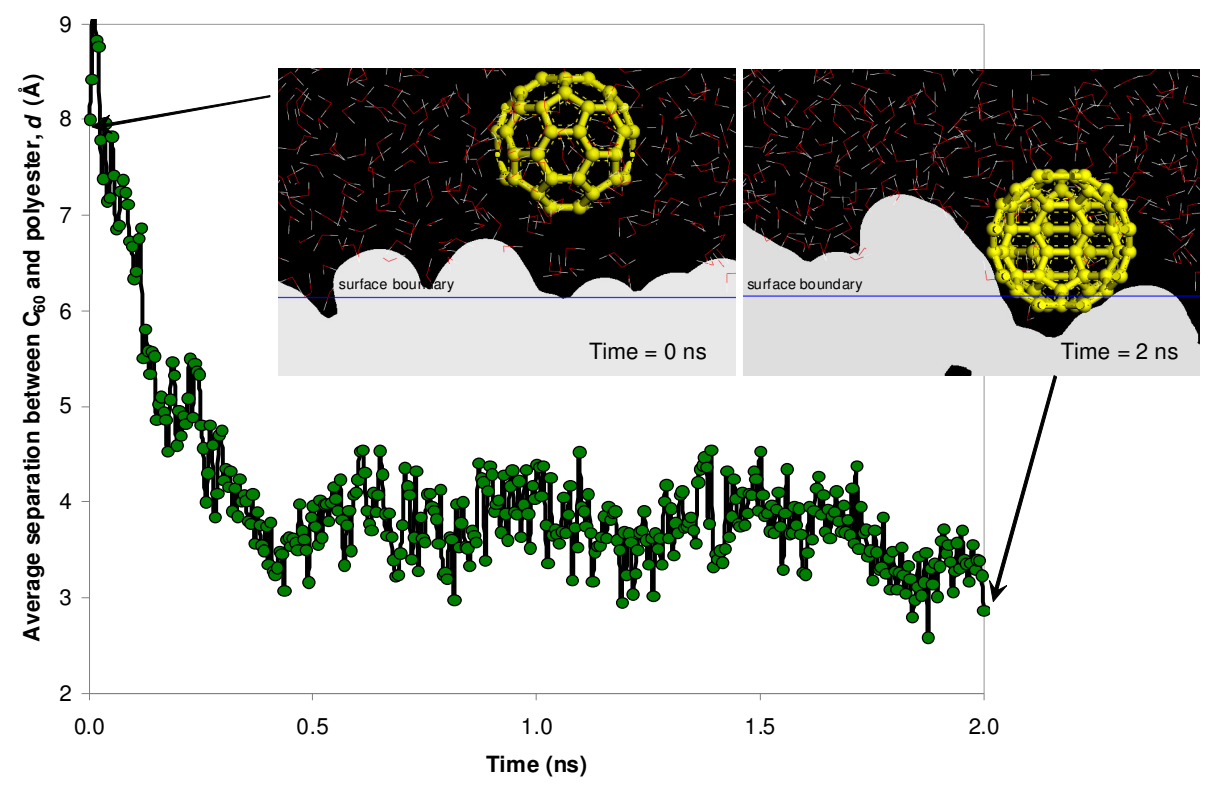

Figure 6.8 Approach curve for a typical Polyester $/ \mathrm{H}_{2} \mathrm{O} / \mathrm{C} 60$ system. Insets show snapshots of initial and final configurations of the system.

The plot depicts the separation $d$ as a function of simulation time. The separation $d$ is defined as the vertical distance between the centre of mass of the fullerene and the defined boundary of the surface shown in the images. Since the fullerene particle has a radius of $\sim 3.7 \AA$, contact between surface and particle is generally achieved when the 
separation $d$, is below $\sim 4 \AA$. It can be seen for this approach curve that the fullerene particle establishes contact with the surface after $0.3 \mathrm{~ns}$ of simulation time. Included in each image, is a cross-sectional slice of the Polyester substrate taken parallel to the $\mathrm{z}-\mathrm{x}$ plane through the centre of mass of the $\mathrm{C}_{60}$ particle. These images show that relaxation leads not only to the movement of $\mathrm{C}_{60}$ toward the surface, but also infiltration of the carbon particle into a shallow cavity, maximizing the contact area of the hydrophobic fullerene with the polymer surface. Clearly, the vdW interaction between the surface and the fullerene remains strong enough to draw the particle onto the surface from short initial separations, despite the presence of water.

In Chapters 4 and 5, we were able to quantitatively calculate the work of separation $\left(W_{\text {sep }}\right)$ between carbon contaminant and polyester surfaces and to relate variations in $W_{\text {sep }}$ to physical and chemical features of the surface via the Hamaker relation ${ }^{13}$ from eq. (4.4). Generally, surfaces or regions with high atomic density exhibit stronger interactions with carbonaceous solids, whereas regions with high atomic surface roughness, which creates zones of lower atomic density, exhibit a weaker interaction with carbonaceous solids. In this section, we will show that this relation also holds true for aqueous systems.

Observation of Figure 6.1 reveals that the structure and the morphology of the polymer surface are not uniform across all five regions with different levels of atomic surface roughness, crosslinking, subsurface density, and depressions or cavities. Table 6.1 depicts a summary of the major characteristics of each region. While there is some variation in these features during the course of each MD run, each location retains its own general characteristics. As a result, the behavior of the fullerene particle is consistent within a single region yet subtly different across the various locations. We therefore present an analysis of typical $\mathrm{C}_{60}$ behavior on each type of surface structure below.

Region A exhibits a high degree of surface roughness on top of a high-density rigid subsurface due to crosslinking. We find that this combination of characteristics in region A provides resistance to penetration of the fullerene. The average equilibrium separation between $\mathrm{C}_{60}$ and polyester $\left(d_{a v}\right)$ in region $\mathrm{A}$ is $3.57 \AA$ and in this particular region, the carbon particle does not completely infiltrate the polyester surface. Presented in Figure 
6.9 are selected radial distribution functions for the interaction between carbon atoms in Polyester to carbon atoms in $\mathrm{C}_{60}\left(\mathrm{~g}_{\mathrm{C}^{\prime}-\mathrm{C}}(\mathrm{r})\right)$. These radial distribution functions provide an indication of the extent of interaction between the fullerene and hydrophobic segments of the polyester. The $\mathrm{g}_{\mathrm{C}^{\prime}-\mathrm{C}}(\mathrm{r})$ for region $\mathrm{A}$ exhibits a reduction in intensity relative to other regions due to a specific combination of densely packed rigid subsurface structure and fine scale roughness on top of this area. Asperities and grooves in region A are sufficiently sparse and significantly smaller than the size of the particle, thus reducing the overall contact area between fullerene and substrate. This is in accordance with the Cassie and Baxter construction ${ }^{57}$ where hydrophobic surfaces can be made superhydrophobic by introducing fine-scale roughness that reduces the contact area between liquid and solid, as in the case of the Lotus leaf. ${ }^{7}$ Our findings are also similar to those of Scardino et al., ${ }^{191}$ who studied the attachment of various algae cells on laser etched polyimide surfaces in static laboratory bioassays. Results showed that the lowest level of attachment occurred where cells were slightly larger than the microtexture wavelength of the surface. Furthermore, while the high density subsurface in region A should lead to a stronger vdW interaction with the particle, it is the rigidity due to crosslinking that prevents infiltration of the $\mathrm{C}_{60}$ into the surface.

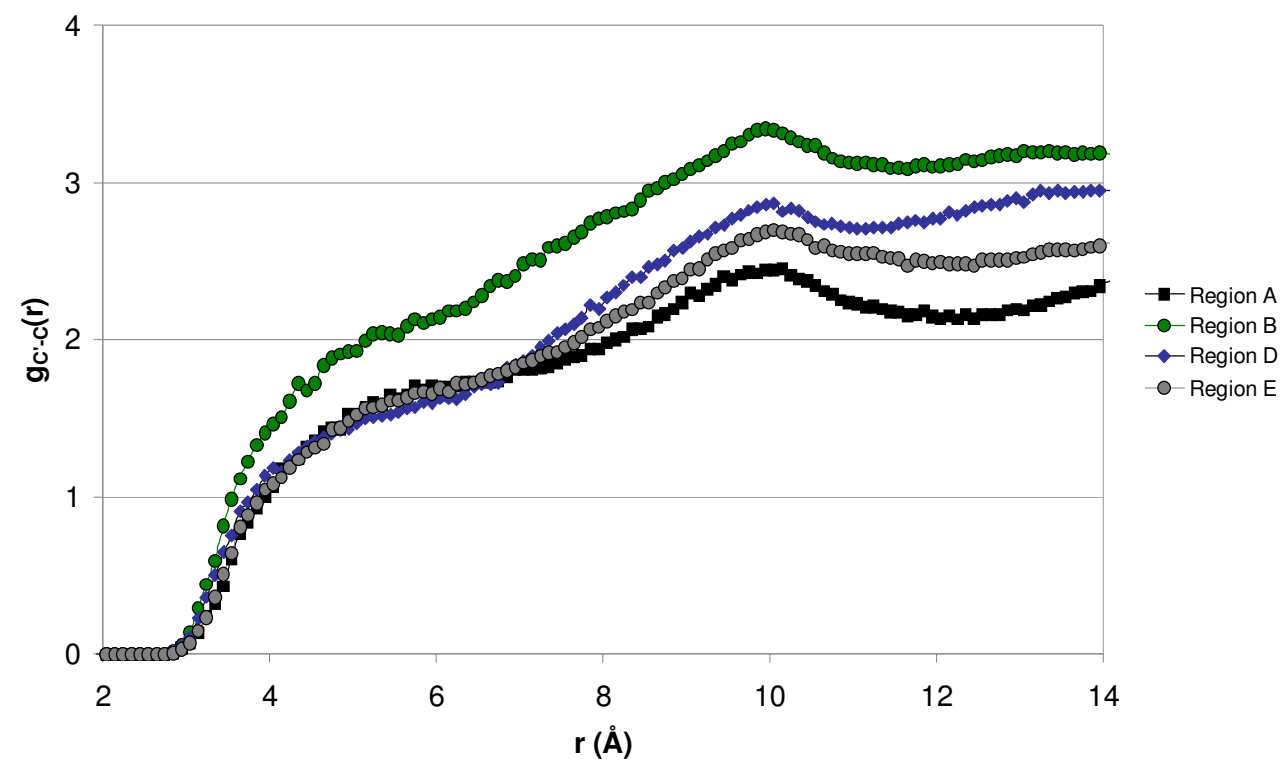

Figure 6.9 Selected radial distribution functions of carbon in polyester (C') and carbon of $\mathrm{C}_{60}$ (C). These radial distribution functions provide an indication of the extent of interaction between the fullerene and hydrophobic segments of the polyester. 
In contrast, region B is characterized by a high density subsurface, punctuated by a combination of very deep as well as quite shallow surface cavities. The deep cavities provide ample opportunity for the fullerene to enter the polyester surface and move through to the bulk of the polymer. The average equilibrium separation in region $\mathrm{B}\left(d_{a v}\right)$ is therefore particularly short (-0.11 $\AA$ ). One mechanism driving the strong attraction in region $\mathrm{B}$ is $\pi-\pi$ interaction between the aromatic carbons of the fullerene and phenyl rings of the polyester. In some locations, this interaction is sufficiently strong to allow the fullerene to penetrate the surface of the polyester which can then lead to the formation of a cage-like arrangement of hydrocarbon chains and aromatic carbon rings surrounding the $\mathrm{C}_{60}$ particle (Figure 6.10). It can be seen from this image that alkyl fragments and aromatic rings surround the sides of the fullerene particle at a distance of 3.5-4.5 $\AA$. In the previous chapter, we showed that relaxation of the polyester in proximity to the hydrophobic carbonaceous surfaces results in the migration of hydrophobic segments from the bulk to the outer surface of the polyester and toward the carbon interface. The vdW attraction between the $\mathrm{C}_{60}$ particle and carbon moieties of the polyester substrate is also observed here. It can be seen that the radial distribution function for region B (Figure 6.9) is characterized by a shift to shorter separations and a significant increase in magnitude compared to other regions on the surface, reflecting the strong hydrophobic interactions.

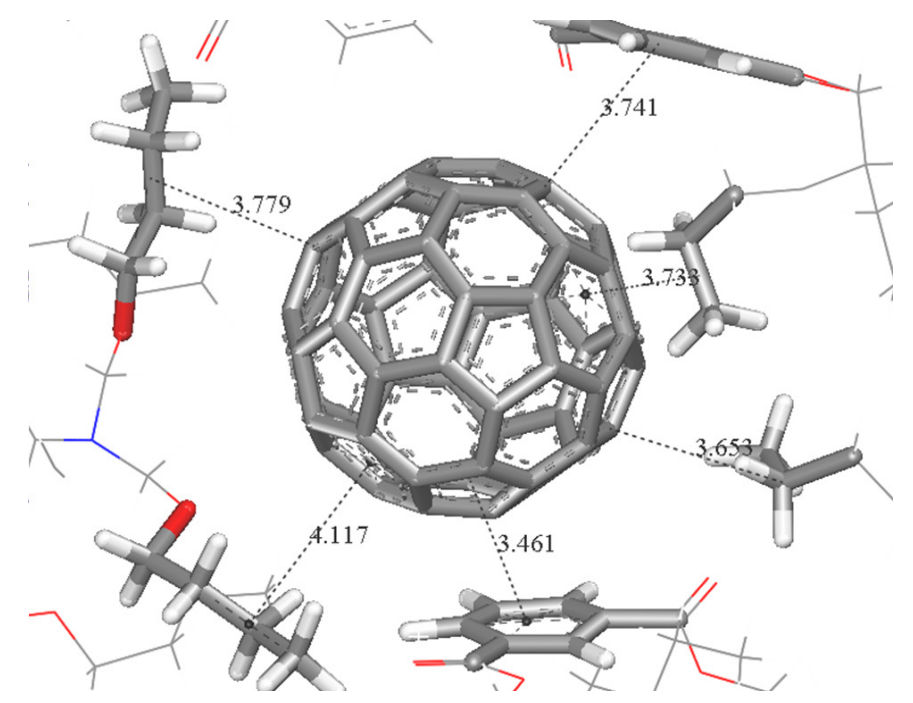

Figure 6.10 A close-up snapshot of fullerene particle inside a polyester layer. The image has been captured during molecular dynamics. It is representative of a typical equilibrium state and highlights a common cage-like formation between hydrophobic segments of the interface. 
The extent to which the $\mathrm{C}_{60}$ particle penetrates the polyester surface may also vary depending on the chemical nature of the underlying surface, specifically, the degree of crosslinking. While the polyester has an overall average crosslinking density of $8.8 \times$ $10^{-4}$ groups $\AA^{-3}$, within the model surface there are areas with significant crosslinking (region C), which are therefore more rigid, and some regions with little or no crosslinking, which exhibit significant mobility at $298 \mathrm{~K}$ (region D). Noticeably, if the $\mathrm{C}_{60}$ particle migrates to region $\mathrm{C}$, which has a high crosslinking density, the extent to which the carbon particle penetrates the polyester surface is reduced $\left(d_{a v}=2.91 \AA\right)$.

A characteristic feature of region $\mathrm{D}$ is a deep but very broad surface depression. Here, the contaminant particle is seen to penetrate the polymer substrate $\left(d_{a v}=0.99 \AA\right)$ as observed for region B. However, region D of the polyester exhibits a much lower atomic density compared to region $\mathrm{B}$. As a result of this, at region D of the surface there is a relatively reduced interaction between fullerene and hydrophobic segments of polyester. The $\mathrm{g}_{\mathrm{C}^{\prime}-\mathrm{C}}(\mathrm{r})$ for region $\mathrm{D}$ (Figure 6.9) exhibits a significant decrease in magnitude compared to that of region $\mathrm{B}$, which can be attributed to the lowered atomic surface density of the polyester in this area.

The most prominent features of region $\mathrm{E}$ are a high subsurface density and a very shallow cavity that the contaminant particle is seen to enter. Because of the absence of surface roughness, interactions between fullerene and hydrophobic segments of the polyester are stronger than those observed in region A of the surface. However, the high subsurface density creates a degree of rigidity such that the fullerene particle rests in the shallow cavity rather than penetrate the bulk of the polymer as commonly observed in regions that feature deeper cavities (e.g., region B). As a result of this, region E gives an average separation intermediate between that of regions A and B. Moreover, the $\mathrm{g}_{\mathrm{C}^{\prime}-\mathrm{C}}(\mathrm{r})$ for region $\mathrm{E}$ (Figure 6.9) exhibits an increase in magnitude compared to that of region $\mathrm{A}$ and a decrease in magnitude compared that of region $\mathrm{B}$.

\subsubsection{Adhesion of $\mathrm{C}_{60}$ on Hydrated Modified Polyester}

In Chapters 4 and 5, we examined the in-vacuo interactions between modified polyester and carbon contaminants, graphite and amorphous carbon. It was shown that chemical modification in the form of hydroxyl or fluorine surface functionalisation can reduce 
adhesion between the carbon contaminant and the polymer surface. The surface modifications offer a strong van der Waals repulsion with the opposing carbon surface, reducing the strength of the adhering contaminant. Similarly, in the presence of water, we find that the extent of infiltration by the contaminant model is reduced by surface modification, and in some areas, the fullerene particle does not come into contact with the modified polyesters. The reason for this is twofold: first, the fluorine and hydroxyl substituents "hold" the fullerene particle at slightly greater separation from the surface, reducing the probability of infiltration, and second, the incorporation of hydroxyl or fluorine substituents to the polyester surface leads to a stronger interaction with water, effectively screening the interaction between $\mathrm{C}_{60}$ and the substrate.

In region $\mathrm{A}$ of the polyester, 25 functional groups $(\mathrm{OH}$ and $\mathrm{F})$ were introduced, achieving a relatively high surface coverage density (Figure 6.1). Here, the $\mathrm{C}_{60}$ particle, initially positioned at $6 \AA$ from the substrate, is seen to be attracted toward the modified polymers as was observed for unmodified polyester. Despite this, the average equilibrium separation between fullerene and the modified polyester surfaces is greater than in the unmodified system by approximately 1-2 $\AA$. Furthermore, the $\mathrm{g}_{\mathrm{C}^{\prime}-\mathrm{C}}(\mathrm{r})$ for these functionalised systems (Figure 6.11a) exhibits a significant decrease in magnitude and shift to larger separation compared to that of their unmodified counterpart. This indicates that as a result of surface modifications, there is a reduction in the overall interaction between fullerene and carbonaceous segments of the polyester. Interestingly, when the MD run is begun with the $\mathrm{C}_{60}$ particle initially positioned at $8 \AA$ above region A of the modified surfaces, we find that the carbon model does not adhere to the substrate but remains suspended in solution. To further confirm this increase in resistance to carbon adhesion, we performed the following computational experiment: (a) isolated the modified systems in their initial configuration, (b) replaced the substituents located in region A of each of the polyester models with hydrogen atoms with re-determination of partial atomic charges to maintain neutrality, and (c) repeated the MD run for these partially "hydrogenated" interfaces. When MD runs were restarted, we observed that the carbon particle is again attracted to these partially hydrogenated surfaces. It appears that a specific combination of chemical and physical surface properties can prevent adhesion of the water-borne $\mathrm{C}_{60}$ particle onto the polymer layer. 


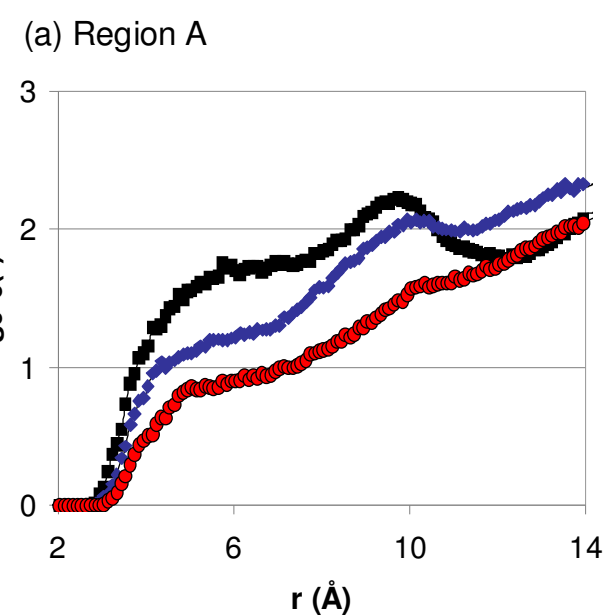

(d) Region D

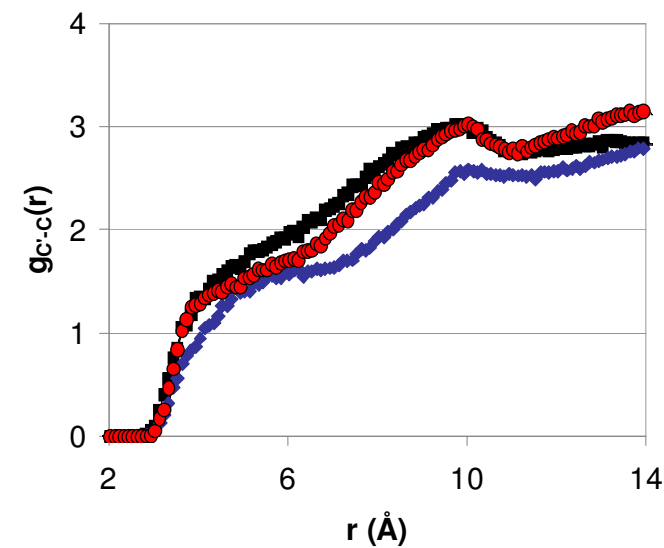

(b) Region B

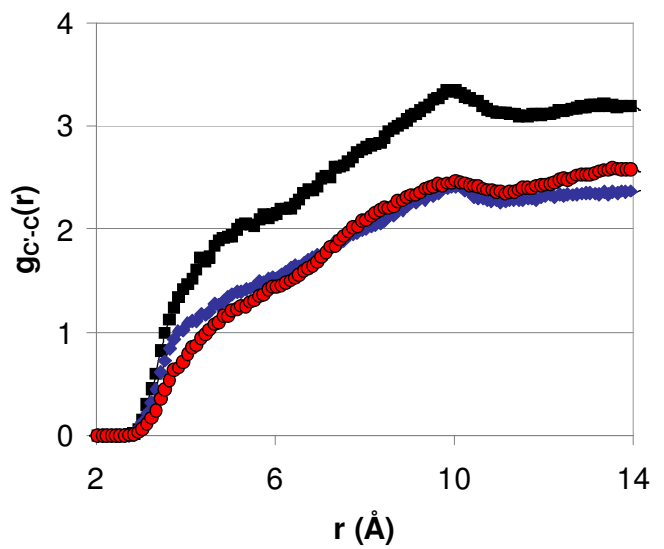

(e) Region E

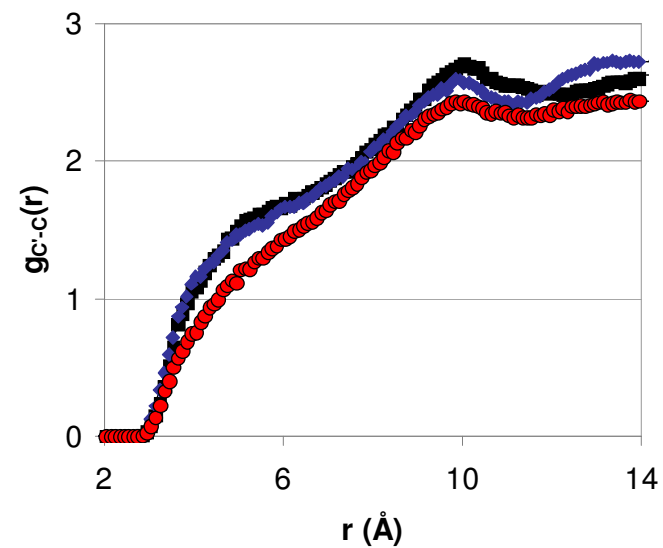

(c) Region C

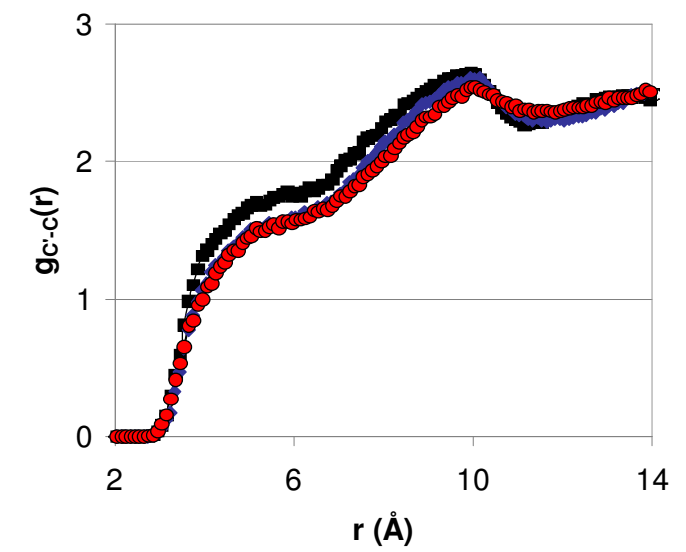

- Polyester75F/Water/Fullerene

- - Polyester75OH/Water/Fullerene

Figure 6.11 Selected radial distribution functions of carbon in polyester (C') with carbon of $C_{60}(C)$. These radial distribution functions provide an indication of the extent of interaction between the fullerene and hydrophobic segments of the polyester. 
Firstly, as a result of chemical modifications, there is a variation in surface/water interactions and local structuring of water molecules (Figure 6.7). This behaviour in conjunction with the inherent resistance of this region due to surface roughness, "rigid" dense subsurface, and lack of porosity, are sufficient to prevent adhesion of the particle onto the surface.

In region $\mathrm{B}$, functional groups were introduced at a considerably lower density than in region $\mathrm{A}$ (Figure 6.1). In region $\mathrm{B}$, the carbon particle is seen to be attracted toward the modified substrates from starting separations of 6-8 $\AA$. Nevertheless, analysis of the 'region B systems' reveals that the carbon particle is held at a larger equilibrium separation from the modified surfaces compared to unmodified polyester $\left(\Delta d_{a v}=4.2-4.3\right.$ $\AA$ ). Moreover, the $\mathrm{g}_{\mathrm{C}^{\prime}-\mathrm{C}}(\mathrm{r})$ for these modified systems (Figure 6.11b) exhibits a significant decrease in magnitude compared to that of their unmodified counterpart, indicating reduced hydrophobic interactions for these modified systems. Therefore, despite adhesion of the carbon particle onto the modified substrates, the presence of surface functional groups reduces the extent of contact between $\mathrm{C}_{60}$ and polyester. This is because of a combination of factors, which include a stronger interaction between the modified substrate and water (Figure 6.4 and Figure 6.5) as well as an increase in the strength of short-range vdW repulsion between functional groups and carbonaceous models compared to that of the original untreated polyester (see Section 4.4.1).

Presented in Figure 6.12 in the following page, are snapshots of typical configurations of Polyester $/ \mathrm{H}_{2} \mathrm{O} / \mathrm{C}_{60}$ systems. Included in each image is a cross-sectional slice taken through the vdW surface of the systems. The slices were generated parallel to the $\mathrm{z}-\mathrm{x}$ plane through the centre of mass of the $\mathrm{C}_{60}$ particle in region $\mathrm{B}$ of the surfaces. The polyester surface in each image is indicated in red and the water layer is indicated in blue. The unmodified model is displayed in Figure 6.12a, and the equivalent hydroxyl modified system is displayed in Figure 6.12b. It can be seen that in the case of the unmodified polyester, Figure 6.12a, the carbon particle adheres to the surface by infiltrating its boundary and opening a cavity. In contrast, for the hydroxyl modified polyester, Figure $6.12 \mathrm{~b}$, water situated underneath the particle reduces the extent of contact between $\mathrm{C}_{60}$ and the substrate. 


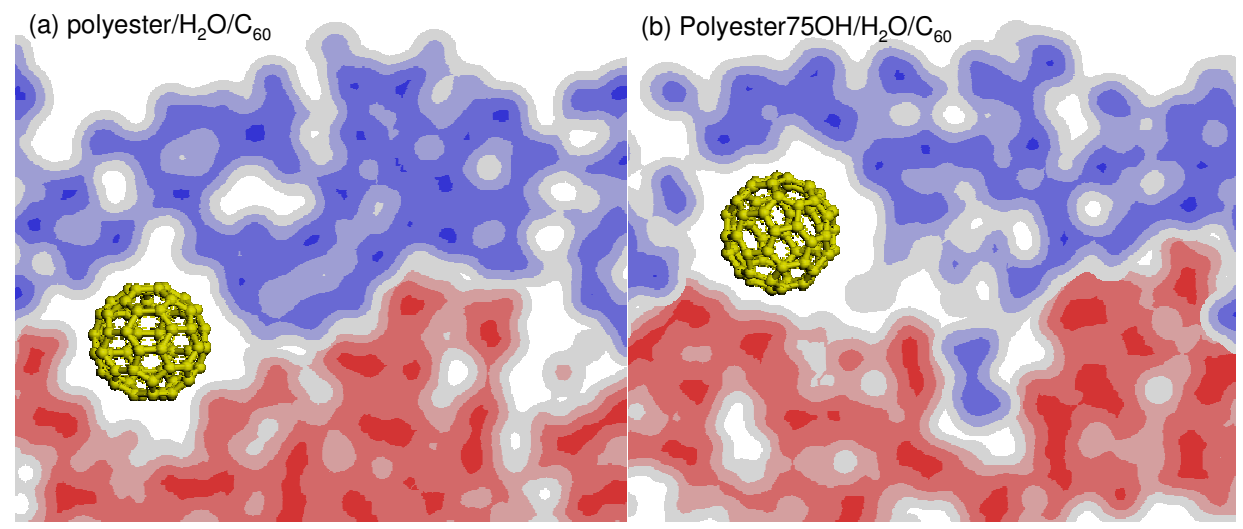

Figure 6.12 A cross-sectional slice taken through the vdW surface of the systems. The polyester surface is indicated in red, the water layer is indicated in blue and the fullerene particle is represented by the yellow, ball and stick structure. Areas of low color intensity along the slice, correspond to regions of reduced atomic concentration while more intense colors, represent regions of increased atomic concentration. (a) In the absence of surface modifications, the fullerene particle is seen to infiltrate the polymer layer. (b) In the case of the hydroxyl modified polymer, water situated underneath the $\mathrm{C}_{60}$ particle, reduces the extent of contact between $\mathrm{C}_{60}$ and the substrate.

A similar behaviour is noted in region D of the surface. For starting separations of 6 $\AA$, modification results in a reduction in interaction between fullerene and carbonaceous segments of the polyester (Figure 6.11d). When the MD run is begun from an initial distance of $8 \AA$ from the modified polyester layer, the carbon model remains suspended in solution. In this instance, surface modification in combination with the large deep cavity that provides sufficient space for water molecules to enter, prevent fullerene from infiltrating the surface.

In contrast, regions $\mathrm{C}$ and $\mathrm{E}$ of the surface are punctuated by cavities that are smaller in size and shallower compared to those along regions B and D. The probability of water filling cavities such as these and preventing the $\mathrm{C}_{60}$ from penetrating the polymer is low, even in the presence of functional groups. Consequently, in these regions, functionalised models do not exhibit the notable increase in resistance to carbon adhesion demonstrated by regions B or D. Here, modification results in only a marginal increase in the average separation between $\mathrm{C}_{60}$ and polyester $\left(\Delta d_{a v}=0.5-0.7 \AA\right)$. The $\mathrm{g}_{\mathrm{C}^{\prime}-\mathrm{C}}(\mathrm{r})$ for these modified systems (Figure 6.11c,e) exhibit minor reductions in magnitude relative to their unmodified counterparts. 


\subsubsection{Effects of Water Layer Thickness on Polyester- $\mathrm{C}_{60}$ Adhesion}

To determine the effect of water layer thickness on the interactions between $\mathrm{C}_{60}$, our contaminant model, and polyester, we generated MD trajectories with water layer thicknesses of $15 \AA$ and $30 \AA$. For a $15 \AA$ thick water layer, and the $\mathrm{C}_{60}$ particle initially located at $\sim 10 \AA$ from the polyester surfaces, we observe that for the majority of MD runs $(\sim 80 \%)$, the contaminant model does not come into contact with the substrates. In these instances, the $\mathrm{C}_{60}$ particle typically moves away from the substrate and within $\sim 1$ ns migrates toward the "air"/water interface, where it remains for the final $1 \mathrm{~ns}$ of the MD simulation. This was also the case for MD runs undertaken for $4 \mathrm{~ns}$. However, when MD simulations were performed with a water layer thickness of $\sim 30 \AA$, the fullerene particle was seen to adhere to the polyester surface even from a significant starting separation of $\sim 10 \AA$. This particular result shows that the hydrophobic particle has a tendency to migrate away from the middle region of the water layer toward the nearest hydrophobic interface, minimising its contact with water.

\subsection{Conclusions}

Using classical molecular dynamics, we studied the interaction of fullerene $\left(\mathrm{C}_{60}\right)$ with modified polyester surfaces in water. We investigated how physical and chemical aspects of the surfaces as well as the interfacial water layer thickness can affect the nature of interaction between polyester and the fullerene. In the absence of chemical surface modifiers, the vdW interaction between fullerene and polyester is sufficiently strong to draw the particle onto the surface, even for a significant initial water layer thickness of $\sim 8 \AA$. However, with the addition of functional groups (F or OH groups) to the polyester surface, there is a clear reduction in the extent of fullerene infiltration. The reason for this is twofold: first, the fluorine and hydroxyl substituents "hold" the $\mathrm{C}_{60}$ particle at slightly greater separation from the surface, reducing the probability of infiltration, and second, the incorporation of hydroxyl or fluorine substituents to the polyester surface leads to a stronger interaction with water, effectively screening the interaction between $\mathrm{C}_{60}$ and the substrate. We suggest that a specific combination of atomic roughness, surface rigidity, and functionalisation can achieve significant reductions in adhesion between polyesters and carbonaceous particles in water. 


\section{Chapter 7}

\section{Effects of Surface Crosslinking on Adhesion and Hardness}

\subsection{Overview}

In this section, we propose a surface crosslinking procedure, aimed at improving the hardness of the outer-region of the polyester coating, and reduce the strength of adhesion between coating and contaminant. We will show how surface crosslinked polyesters may be modelled and present an in-silico nanoindentation experiment whereby nanoparticles, fullerene in this case, are used to probe different regions of the polymer surface enabling us to characterize the surface hardness of the polymer film and calculate the work of adhesion between polymer and nano-particle $\left(\mathrm{C}_{60}\right)$. We will show that surface crosslinking of the polymer with isophorone di-isocyanate molecules, improves the stability of the coating's outer surface leading to significantly weaker adhesion with our $\mathrm{C}_{60}$ contaminant model. 


\subsection{Introduction}

Crosslinking involves the bonding of polymer chains with one another, and is in practice, achieved during the curing process of the paint. ${ }^{197,198}$ Such a procedure usually increases the density of the polymer, limits the mobility of the chains, and results in an increase in rigidity of the material. When the crosslinks are formed predominantly near the surface region of the polymer, the process is referred to as surface crosslinking. It has been suggested that surface crosslinking may limit the reorientation of surface groups and prevent aging effects. ${ }^{158}$ In one example of surface crosslinking, Hyun et al. ${ }^{159}$ irradiated PET films with $\mathrm{Ar}^{+}$ion beam prior to $\mathrm{O}_{2}$ plasma surface modification, in an attempt to reduce polymer chain mobility and hydrophobic recovery of the samples through crosslinking. Spectroscopic ellipsometry, AFM and FTIR were used to characterise the composition of the surfaces and contact angle measurements were undertaken to evaluate the degree of hydrophobic recovery. Results showed that $\mathrm{Ar}^{+}$ion beam irradiation through radical-radical coupling induces surface crosslinking of the PET films. Moreover, this crosslinking pre-treatment was shown to reduce hydrophobic recovery of the $\mathrm{O}_{2}$ plasma treated samples. Similar experimental studies of Tretinnikov ${ }^{199}$ and Fisher ${ }^{200}$ have shown that irradiation of polymer materials with electron beams and alpha particles respectively, can induce surface crosslinking. Both of these studies showed that radiation induced crosslinking occurs concurrently with polymer chain scission, also know as fragmentation. Whilst fragmentation can lead to an increase in the molecular mobility of the polymer, in these specific examples, indentation tests have shown the surface crosslinking results in an overall increase in the hardness values of the polymer samples. Hence, the effect of fragmentation is largely offset by the presence of surface crosslinking. Aside from irradiation, chemical formulation has also been used to construct surface crosslinked polymer coatings. Lukey et al. $^{201}$ formulated crosslinked polyester coatings with varying amounts of hexamethoxymethylmelamine (HMMM). XPS analysis has shown that for high melamine content polyesters, the HMMM crosslinker is concentrated in the upper layers of the samples rather than homogeneously distributed throughout. These high melamine content samples also exhibited reduced adhesion with zirconia particles compared to 
their low melamine content counterparts. It was suggested that the lower adhesion arises from reduced contact as a result of the less deformable asperities of the crosslinked melamine enriched surface layer.

Yarovsky and Evans ${ }^{94}$ presented a detailed methodology for constructing realistic models of crosslinked polymer resins using classical molecular dynamic (MD) simulations. This method involves packing a mixture of polyester chains and crosslinker molecules in experimental mole ratio in a periodic cell. Following equilibration of the mixture, reactive sites within a suitable cut-off radius are identified and new covalent bonds formed between these sites. The process of equilibration and bond formation is continued until no further change in the crosslinking density is observed. Results show that the accessibility of the reactive sites affects the number of crosslinks formed, and that the size of the crosslinker molecules affects their mobility to reach the reactive sites. Hein et al. ${ }^{96}$ also constructed crosslinked networks of poly(dimethylsiloxane) (PDMS) via molecular dynamics. Starting from a united atom model of an equilibrated melt of PDMS chains, tetrakis(dimethylsiloxy)silane crosslinkers were attached to terminal $\mathrm{OH}$ groups of the polymer that were within a short capture distance, similar to the method described by Yarovky and Evans. ${ }^{94}$ In turn, the elastic moduli of the crosslinked polymers were determined by uniaxial elongation of each network. The results were in qualitative agreement with experimental measurements of model end-linked PDMS networks. More recently, Wu et al. ${ }^{76}$ described a similar method to construct atomistic models of crosslinked polymers. They calculated the density and elastic constants of the crosslinked systems which compare well with experimental data.

However, in each of these theoretical studies, crosslinking is carried out on the bulk of the polymer resulting in polymer models where the crosslinks are distributed throughout the samples rather than specifically concentrated in the surface region. In this chapter, we present a methodology to chemically surface crosslink the polyester coating with hexamethylene di-isocyanate (HDI) and isophorone di-isocyanate (IPDI) molecules respectively. The aim of this is to reduce the mobility of the topmost region of the coating, but keep the mobility of the core region unperturbed. We subsequently present a series of molecular dynamics simulations of nano-identation that occurs as a fullerene particle is brought onto these polyester films. Force versus separation curves generated 
during loading of the 'spherical' $\mathrm{C}_{60}$ particle indenter, are used to determine the hardness of the polyester coatings. Both the hardness of the outermost and sub-surface regions of the coatings are evaluated. We also determine, through in-silico nanoindentation, the work of adhesion between polyester and $\mathrm{C}_{60}$, our soot contaminant model. This enables us to examine the influence of surface crosslinking on the contaminant-sheilding behaviour of the coating.

\subsection{Molecular Models and Theoretical Procedures}

\subsubsection{Composition of Molecular Models}

The polyester model under consideration has been described in detail in Section 3.1. The polyester chains of the model have been previously crosslinked resulting in a structure with a crosslinker density of 0.31 crosslinkers $\mathrm{nm}^{-3}$, an overall density of $1.14 \mathrm{~g} \mathrm{~cm}^{-3}$. However, the crosslinks are randomly distributed throughout the structure, and so the polymer chains at the surface of the film still exhibit a high degree of mobility at ambient temperature (see Chapter 5 on aging effects).

For this particular investigation, we have chosen two di-isocyanate molecules as potential surface crosslinkers: hexamethylene-1,6-di-isocyanate (hexamethylene diisocyanate, HDI) and 3-isocyanatomethyl-3,5,5-trimethylcyclohexylisocyanate (isophorone di-isocyanate, IPDI). These di-isocyante molecules are presented in Figure 7.1 in the following page. Also included in this figure, is the chemical formula of the related crosslinking reaction, whereby isocyanate molecules, denoted R-NCO, react with alcohols on the polymer chain to form the crosslinks. Due to the lack of hydroxyl groups $(\mathrm{OH})$ on the surface of the polyester model, we functionalised the polyester surface with $\mathrm{OH}$ groups as described in Section 3.1, simulating an oxidation via UV/ozone process. We introduced 75 hydroxyl groups per unit cell achieving a surface coverage of $4.7 \mathrm{OH}$ groups per $\mathrm{nm}^{2}$. In addition to the polymer crosslinking reaction described above, a selfcrosslinking reaction may also take place, however to maintain computational tractability, self-crosslinking of isocyanates was not considered. 

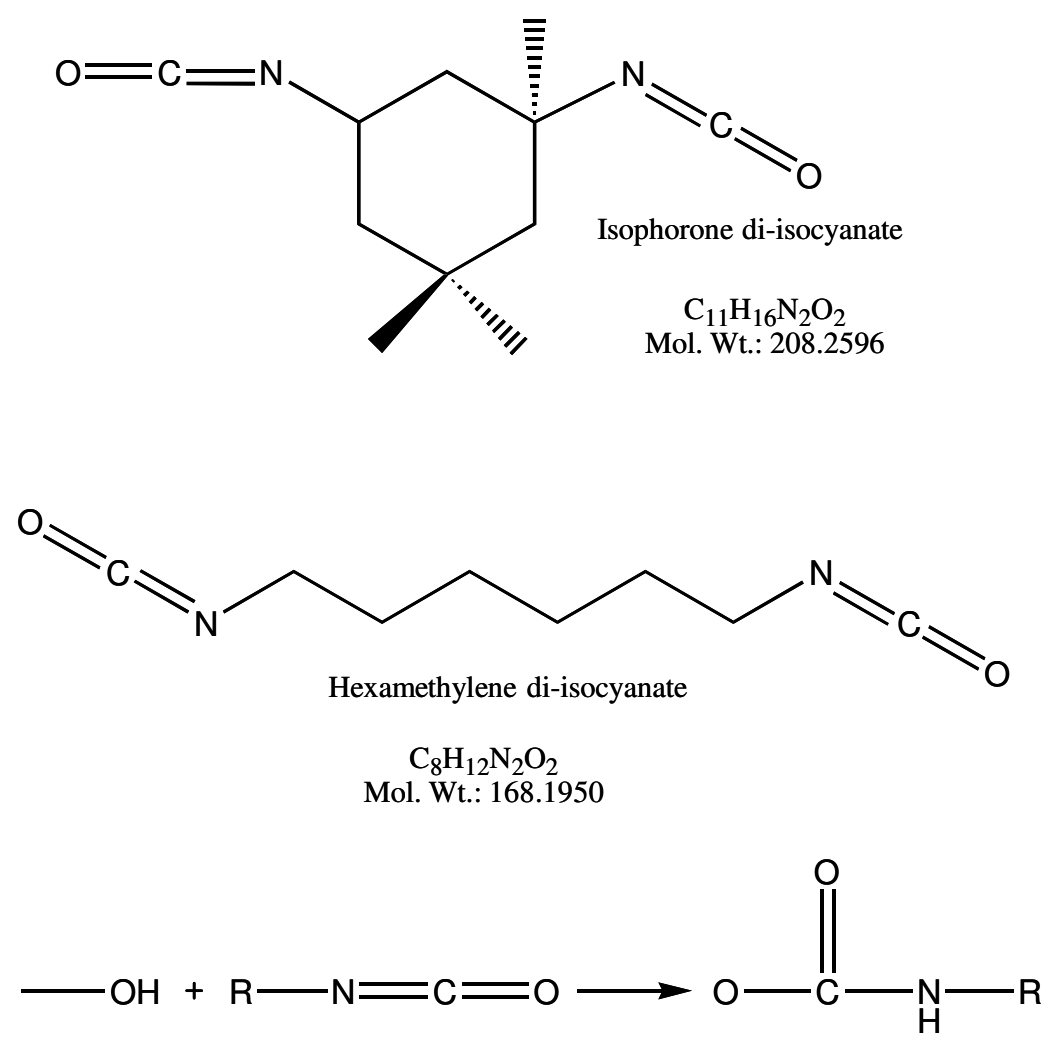

Figure 7.1 Chemical composition of the di-isocyanate crosslinking molecules and the chemical formula of the related crosslinking reaction where $R=\left(\mathrm{CH}_{2}\right)_{6}$ and $\mathrm{R}=\mathrm{C}_{10} \mathrm{H}_{8}$ for Hexamethylene di-isocyanate (HDI) and Isophorone diisocyanate (IPDI) crosslinkers respectively.

The Amorphous Cell procedure, which incorporates some features of the original Theodorou and Suter approach ${ }^{202}$ and the Meirovitch scanning method, ${ }^{203}$ was used to construct a layer of each crosslinker molecule. We inserted forty HDI molecules into a cell of dimensions $40 \times 40 \mathrm{x} \sim 7 \AA$ to create a liquid layer with the experimental density of $1.05 \mathrm{~g} \mathrm{~cm}^{-1}$. The procedure was repeated for IPDI molecules to give a liquid layer, with a unit cell dimensions of $40 \mathrm{x} 40 \mathrm{x} \sim 9 \AA$ A and known density of $1.06 \mathrm{~g} \mathrm{~cm}^{-1} .^{204}$ The number of di-isocyanate molecules included in each liquid layer was selected so as to obtain a 1.1:1.0 mole ratio of isocyanate groups ( $\mathrm{NCO})$ to polyester hydroxyl groups $(\mathrm{OH})$ in accord with experimental studies. ${ }^{197,198,205}$ Each crosslinker layer was equilibrated by undertaking molecular dynamics in the NVT ensemble for $4 \mathrm{~ns}$. 


\subsubsection{Modelling Surface Crosslinking of Polymers}

Experimentally, polymer crosslinks are achieved by mixing a solution of the polymer with a solution of crosslinker. ${ }^{197,201,204-206}$ During curing, the mixture is usually cast onto metal panels or moulds and allowed to crosslink. In the case of di-isocyanate crosslinking, the curing process can occur at ambient conditions ${ }^{197,198}$ as well as higher temperatures. ${ }^{204-206}$ This chemical approach generally results in crosslinked samples where the crosslinks are randomly distributed throughout the polymer samples rather than specifically concentrated in the surface region. In this study, we simulate an analogous procedure to construct surface crosslinked polyester models. The procedure involves several steps described below in full detail:

Step 1: The polyester-crosslinker systems were initially prepared by combing the hydroxyl modified polyester with each amorphous crosslinker layer in a $3 \mathrm{D}$ periodic cell of $\mathrm{x}, \mathrm{y}$ and $\mathrm{z}$ dimensions of $40 \mathrm{x} 40 \mathrm{x} 50 \AA$, respectively. Each cell was then extended along the $\mathrm{z}$ axis to create a vacuum layer approximately $15 \AA$ thick, which ensures that interactions between adjacent cells in the $\mathrm{z}$ direction are neglible, resulting in quasi-2D periodic systems.

Step 2: Each polyester-crosslinker system was then equilibrated by undertaking MD simulations in the NVT ensemble for $1 \mathrm{~ns}$ at $298 \mathrm{~K}$, in accord with experimental crosslinking conditions. ${ }^{197}$

Step 3: Following equilibration, the polyester - crosslinker systems were analysed to identify isocyanate and hydroxyl groups in sufficient proximity to undergo reaction. The distance of $4 \AA$ between carbon (C) of the isocyanate group and oxygen of polyester (O) was used as a reaction cutoff distance, i.e, C-O pairs separated by a longer distance were not considered reactive. Although the choice of cutoff distance is somewhat arbitrary, it was based on several trials of various distances and was considered the most appropriate to achieve a crosslinker surface concentration that is comparable to that attained by experiment. ${ }^{201}$ In particular, the ratio of nitrogen of isocyanate to carbon is 0.09 within a surface depth of $12 \AA$ for both crosslinked models.

Step 4: The sites identified in the previous step were chemically reacted, with new bonds formed, during which the hydrogen atom of the hydroxyl oxygen was transferred to the isocyanate nitrogen atom. The resultant molecular systems were then energy 
minimised, relieving any strain due to the formation of new bonds and re-equilibrated using molecular dynamics in the NVT ensemble (1ns).

Step 5: Steps three and four were repeated until no further crosslinking reactions were possible (i.e. no more reactive sites were identified within the selected reaction radius). When the crosslinks were formed and the construction terminated, all excess species were removed from the systems. These include unreacted hydroxyl groups of the surface, as well as di-isocyanate molecules with either or both of their isocyanate groups unreacted during crosslinking. This approach enables us to isolate the effect of surface crosslinking on the mechanical properties of the film, by removing complexities associated with dangling crosslinker molecules, which create highly irregular surface profiles and increase surface polarity. Also, pendent crosslink molecules are expected to have negligible effects on the hardness of the film compared to bonded crosslinks. The resulting sites were then capped with hydrogen atoms, and the final crosslinked samples were energy minimised removing any unfavourable interactions caused be the deletion of excess molecules. For the polyester-hexamethylene model (Polyester15HDI), a maximum of 15 HDI molecules were attached per unit cell surface to achieve a surface coverage of 1.1 crosslinker molecules per $\mathrm{nm}^{2}$. In the case of the polyester-isophorone model (Polyester14IPDI), a maximum of 14 IPDI molecules were attached per unit cell surface, leading to a surface coverage of 1.0 crosslinker molecule per $\mathrm{nm}^{2}$.

Step 6: In order to enable shrinkage (volume reduction) of the surface that may occur during the curing process, molecular dynamics was undertaken for $3 \mathrm{~ns}$ in the NPT ensemble. Atmospheric pressure was applied to the atomistic model surface, at a (curing) temperature of $298 \mathrm{~K}$, so that the densities of the layers reached their equilibrium values.

\subsubsection{Modelling Nanoindentation}

To explore the effects of surface crosslinking on hardness, the crosslinked polyester surfaces (Polyester15HDI and Polyester14IPDI) were analysed by a simulated nanoindentation process in comparison with the base polyester (Polyester). The $\mathrm{C}_{60}$ fullerene was chosen as the indenter particle, as it has a higher elastic modulus than the material being tested, which is a requirement for indentation experiments. In the 
previous chapter, we also used $\mathrm{C}_{60}$ as a model for soot contaminant particle, and therefore adhesion values determined between polyester and $\mathrm{C}_{60}$ during indentation can provide a measure of contaminant adhesion as well. Prior to indentation, each of the three samples were placed on top of a fixed graphene layer in a simulation cell of size $36.90 \times 38.35 \times 60.00 \AA$, and equilibrated by undertaking MD simulations in the NVT ensemble for $2 \mathrm{~ns}$. The graphene layer is used to mimic a rigid substrate that supports each surface sample during indentation.

Indentation (loading) was then performed by simulating the approach of the 'spherical' $\mathrm{C}_{60}$ indenter towards the surface in the $\mathrm{z}$ direction (the normal to the plane of the surface) as shown in Figure 7.2. The $C_{60}$ particle was initially positioned at $\sim 14 \AA$ away from the surface where the interaction between the surface and the particle is negligible. The $\mathrm{C}_{60}$ particle was then displaced by $2 \AA$ steps towards the surface. At each carbon-surface separation, molecular dynamics was undertaken in the NVT ensemble for 500 ps with carbon atoms of the $\mathrm{C}_{60}$ indenter and graphene substrate held rigid while the polymer was free to relax. The interaction energy (potential) between carbon and surface was monitored during molecular dynamics. The following quantities were averaged over the final 250 ps: (1) the force $F_{z}$, on the $\mathrm{C}_{60}$ particle along the $\mathrm{z}$ direction, which was calculated by taking the derivative of the potential between $\mathrm{C}_{60}$ and surface as function of the $\mathrm{z}$ coordinates (2) the separation $d$, between $\mathrm{C}_{60}$ and surface, which was evaluated by averaging all vertical distances between each $\mathrm{C}_{60}$ carbon atom and its nearest polyester surface atom. As the particle approached the surface, the step size was reduced to ensure that the indenter speed is sufficiently slow to ensure equilibration. When the $\mathrm{C}_{60}$ particle was brought to an average separation of $4.45 \AA$, the step size was reduced to $1 \AA$. Then from $2.16 \AA$, the step size was further reduced to $0.75 \AA$. We note that, as the particle was displaced toward the surface, there was an obvious reduction in the separation value $d$. However, the magnitude of displacement (the step size) is always larger than the reduction in $d$, because the polymer is highly deformable and its surface molecules can move to meet the fullerene particle. For each surface sample, Polyester, Polyester15HDI and Polyester14IPDI, five separate indentations were carried out, with the $\mathrm{C}_{60}$ particle driven into different regions of the surfaces. The regions are displayed in Figure 6.1. Data of force $F_{z}$ versus separation $d$ were generated. The data was fitted 
through linear interpolation and averaged across the five regions to obtain a single load versus separation plot for each sample.

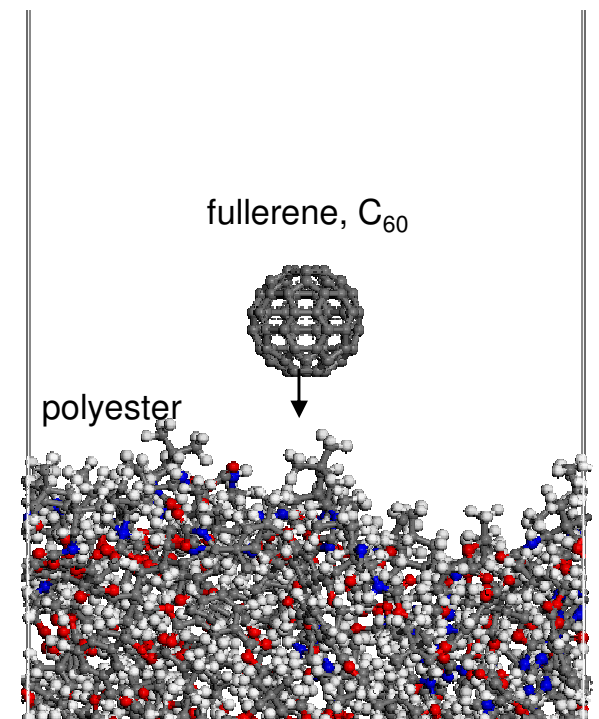


avoid 'double counting' during calculation of the contact area, a coefficient of one half was employed. Using the Connolly algorithm ${ }^{144}$ the surface area of each structure was evaluated by obtaining the area of the surface at the boundary between a probe of radius $1.7 \AA$ and the vdW radii of the atoms in the structure, in externally accessible regions only.

From equations (7.1) and (7.2), we evaluated the hardness of the model surfaces at maximum load values $\left(F_{\max }\right)$ of $0.3 \mathrm{nN}$ and $0.6 \mathrm{nN}$. The reason for this was to be able to quantify the hardness of the outermost and sub-surface regions of the coatings. Hardness measurements undertaken at $0.3 \mathrm{nN}$ resulted in very small indentation depths (less than 1 $\AA$ ) and therefore represent the average hardness of the topmost region or exterior of the surface. In comparison, hardness measurements undertaken at $0.6 \mathrm{nN}$ resulted in larger indentation depths (greater than $1 \AA$ ) and represent the average hardness of the subsurface region of the coating. We note that measurements of hardness and other mechanical properties at such small length scales (typically at the nanometer level) can lead to values that are considerably different from the experimentally determined macroscale values of the material. ${ }^{208,209}$ Furthermore, at such small loads, the observed value of hardness is dependent on the depth of indentation. However, such measurements enable a comparison of the relative hardness of different surfaces.

To examine the effect of surface crosslinking on adhesion, we evaluated the adhesion energy $\left(E_{a d h}\right)$ between the $\mathrm{C}_{60}$ particle and each polyester sample, by integrating the force plots generated during indentation.

$$
E_{a d h}=\frac{\int_{\infty}^{-\infty} F_{z} d z}{A_{C 60}}
$$

From plots of adhesion energy versus separation we determined the equilibrium separation $d_{0}$, and work of adhesion $W_{\text {adh }},{ }^{32,143,210}$ (see eq. (1.2)) between polyester and $\mathrm{C}_{60}$.

\subsubsection{Computational Details}

Energy minimisation was carried out using the conjugate gradient procedure at a convergence criterion of $0.01 \mathrm{kcal} / \mathrm{mol} / \AA$ A. During minimisation, the non-bonded 
interactions were calculated using the Ewald procedure with an accuracy of 0.01 $\mathrm{kcal} / \mathrm{mol}$ and an update width of $1.0 \AA$. For molecular dynamics procedures and to retain computational tractability, non-bonded interactions were calculated using the atombased summation method, with a cut-off radius of $15.5 \AA$, a spline width of $5 \AA$ and a buffer width of $2 \AA$. A long range vdW tail correction was also applied for non-bonded interactions larger than the cut-off radius.

A 1.0 fs time step was used for NPT and NVT dynamics. The Andersen thermostat (see Section 2.9) was employed to control the temperature with a collision ratio of 1.0. For NPT dynamics, the Berensden barostat (see Section 2.10) was used to maintain a pressure of $1 \mathrm{~atm}$ with a pressure decay constant of $0.10 \mathrm{ps}$. All systems were equilibrated by ensuring that no free energy drifts occur during molecular dynamics. A minimum of 250 ps was required to reach equilibrium with some systems requiring significantly longer times (up to $4 \mathrm{~ns}$ ). The force $F_{\mathrm{z}}$ was averaged over the final $250 \mathrm{ps}$ of dynamics, whilst the separation $d$ and the contact area $A$ were averaged over 50 ps of dynamics.

\subsection{Results and Discussion}

\subsubsection{Surface Crosslinking of Polyester films}

The crosslinking reaction considered in this study involves isocyanate groups (NCO) of hexamethylene di-isocyanate (HDI) or isophorone di-isocyanate (IPDI) that react with hydroxyl groups along the polyester surface (see Figure 7.1). Figure 7.3a depicts the percentage of reacted hydroxyl $(\mathrm{OH})$ groups as a function of time during the simulated crosslinking process, allowing us to qualitatively assess the rate of this reaction from the slope of the curve. It is clear from Figure 7.3a that in both cases of crosslinking, the rate of the reaction decreases as crosslinking proceeds. Also, the rate of the reaction is generally independent of the type of di-isocyanate molecule used, since both curves exhibit a very similar shape. Lastly, we find that $\sim 61$ to $67 \%$ of $\mathrm{OH}$ groups along the polyester surface participate in the reaction, with $\sim 33$ to $39 \%$ of $\mathrm{OH}$ groups remaining unreacted. 
(a)

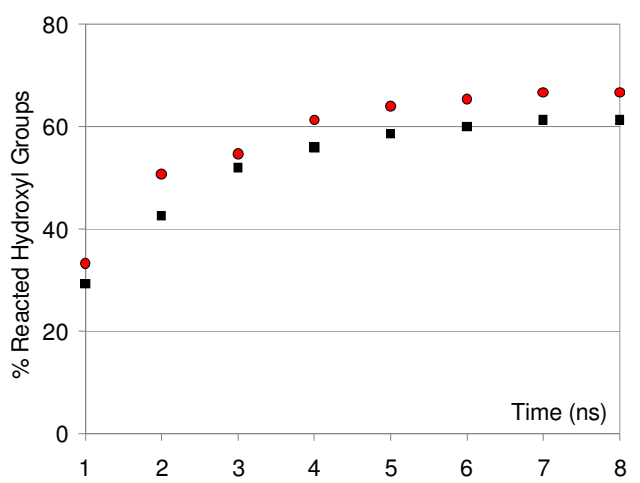

(c)

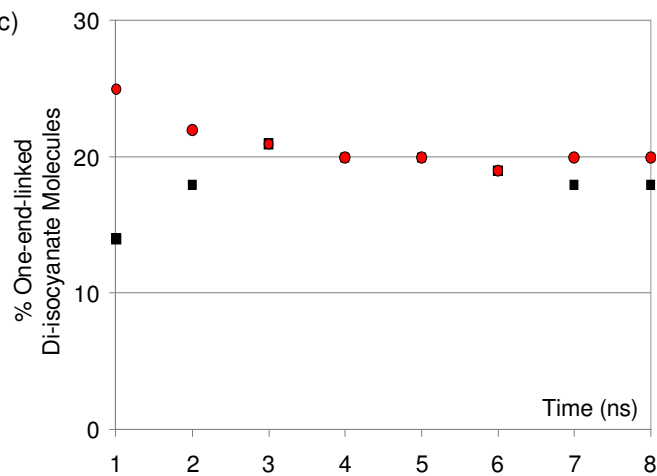

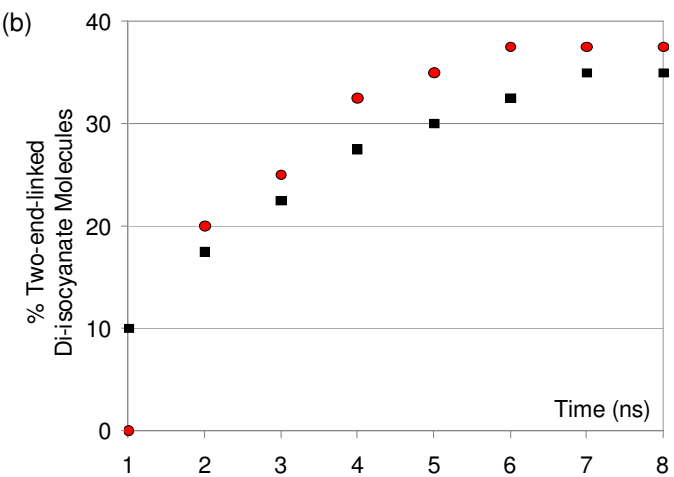

- Hexamethylene di-isocyanate (HDI) crosslinking - Isophorone di-isocyanate (IPDI) crosslinking

\section{Figure 7.3 (a) Percentage of reacted $\mathrm{OH}$ groups during crosslinking (b) Percentage of crosslinks formed during crosslinking (c) Percentage of dangling residues during crosslinking.}

The formation of crosslinks within the samples was monitored by evaluating the percentage of two-end-linked di-isocyanate molecules during crosslinking Figure 7.3b. A two-end-linked di-isocyanate molecule has both of its isocyanate groups reacted to the surface of the polyester. The most striking feature of Figure $7.3 \mathrm{~b}$ is that at the start of the crosslinking process, within the first nanosecond, there is a significant difference in crosslink formation between the two samples. In particular, the hexamethylene crosslinking curve exhibits a delay followed by a rapid rise in the percentage of two-endlinked di-isocyanate molecules at $2 \mathrm{~ns}$. This feature is not observed in the isophorone diisocyanate crosslinking curve which exhibits a more immediate rise in crosslink formation. This can be attributed primarily to the difference in structure between the two di-isocyanate molecules. When the polyester surface is first exposed to the linear HDI molecules, we find that the majority of HDI crosslinkers align perpendicular to the plane of the surface. Therefore when this arrangement is equilibrated, and appropriate sites 
chemically reacted, HDI molecules that are attached to the surface stick out from the polyester forming dangling residues. The dangling molecules are referred to as one-endlinked HDI molecules. Figure $7.3 \mathrm{c}$ displays the percentage of one-end-linked diisocyanate molecules during crosslinking. Expectedly, the plot indicates that within the first nanosecond of the crosslinking process, there is a high percentage of dangling HDI molecules. As crosslinking proceeds and the system adjusts to the new composition, the unreacted ends of the dangling residues bend or drop towards the surface forming additional reaction sites with $\mathrm{OH}$ groups of the polyester. When these sites are chemically reacted, we observe a sharp increase in the percentage of two-end-linked HDI molecules (Figure 7.3b) and reduction in the percentage of one-end-linked HDI molecules (Figure 7.3c). This behaviour explains the delay and rapid rise in the percentage of crosslinks, but in addition to this, demonstrates the significant flexibility exhibited by HDI molecules during initial crosslinking of the polyester surface. In contrast, the cyclic IPDI molecules have a more compact molecular structure relative to HDI molecules. Also important is the fact that the isocyanate groups of isophorone diisocyanate are separated by a shorter distance $(\sim 6 \AA)$ compared to isocyanate groups of hexamethylene di-isocyanate $(\sim 12 \AA)$. The reduced spacing between isocyanate pairs leads to a greater probability that the two isocyanate groups of a given IPDI molecule, will react with the surface concurrently. For this reason, we observe a reduced number of dangling residues (Figure 7.3c) and a greater portion of two-end-linked residues during (Figure 7.3b) the initial stage of isophorone di-isocyanate crosslinking. However, aside from this difference Figure $7.3 \mathrm{~b}$ shows the rate of crosslink formation is generally independent of the type of di-isocyanate molecule used. A maximum of $15 \mathrm{HDI}$ and 14 IPDI crosslinks were formed in the hexamethylene and isophorone crosslinked surfaces, respectively.

On completion of the surface crosslinking process, the atomic density of each polyester sample was evaluated. We found that the atomic density values of the hexamethylene crosslinked polyester (Polyester15HDI), and isophorone crosslinked polyester (Polyester14IPDI), were $\sim 5 \%$ and $\sim 2 \%$ lower than the density of the original polyester, respectively. The reason for this reduction in atomic density is due to 
protruding crosslinker fragments that accentuate the atomic roughness of the polyester film.

\subsubsection{Force plots of polyester- $\mathrm{C}_{60}$ systems}

By lowering the $\mathrm{C}_{60}$ particle (fullerene) in a step-wise manner onto the surface and monitoring the force acting on the particle along the $\mathrm{z}$ direction, plots of force $\left(F_{\mathrm{z}}\right)$ versus separation were generated (Figure 7.4). The evolution of the surfaces was also monitored, by taking $2 \mathrm{D}$ cross-section images of the structures at various separations during indentation for a single region of the surface. Images of the indentation of the unmodified polyester surface (Polyester - $\mathrm{C}_{60}$ ) are shown in Figure 7.5 in the following page. Each 2D image comprises five snapshots depicting the vdW surface of the equilibrated structure, over-layed onto one another. The snapshots have been taken through the centre of the indenter parallel to the $\mathrm{x}-\mathrm{z}$ plane.

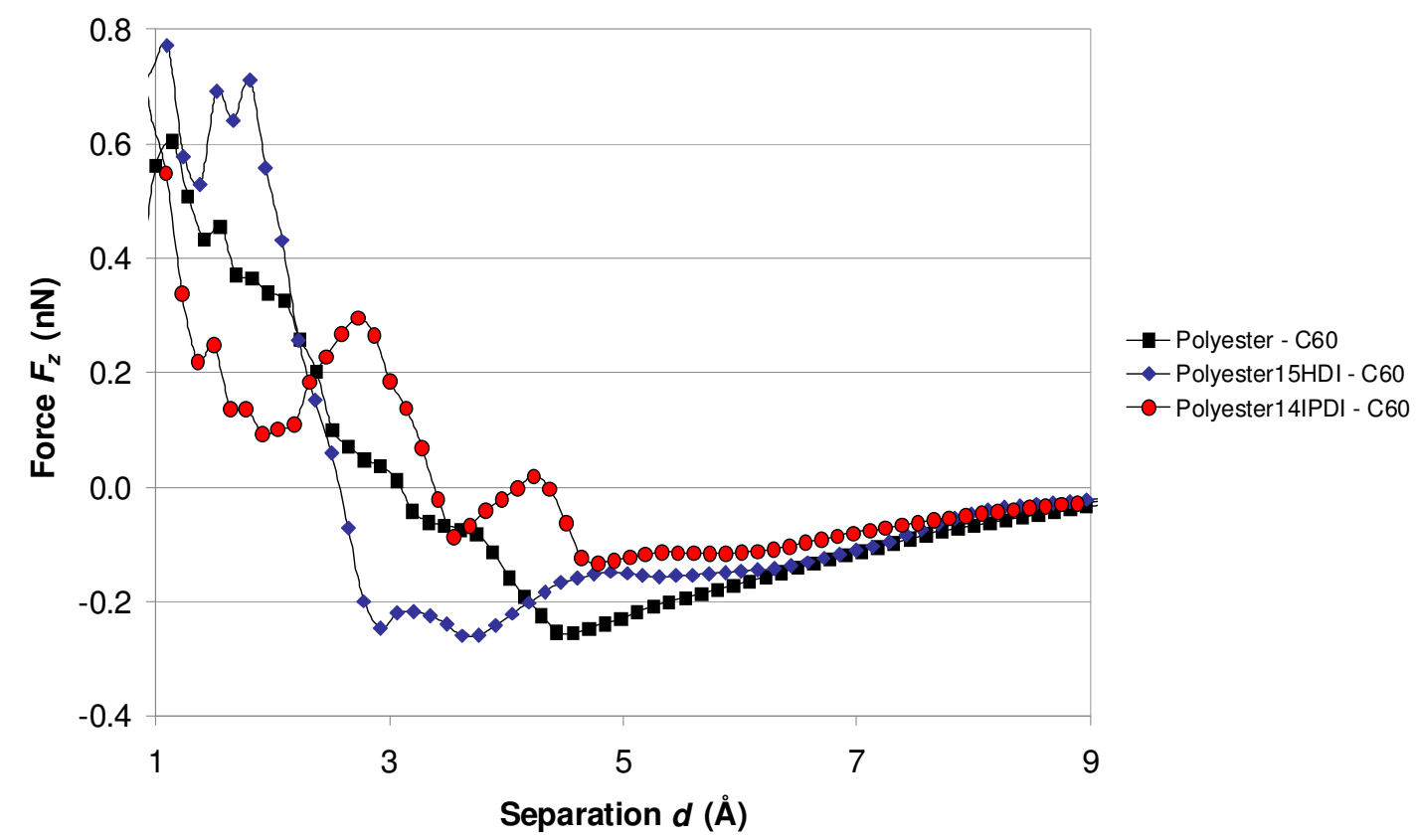

Figure 7.4 Plots of the normal force on the $\mathrm{C}_{60}$ particle versus the average distance between $\mathrm{C}_{60}$ and surface. 
(a) $d=8.23 \AA$

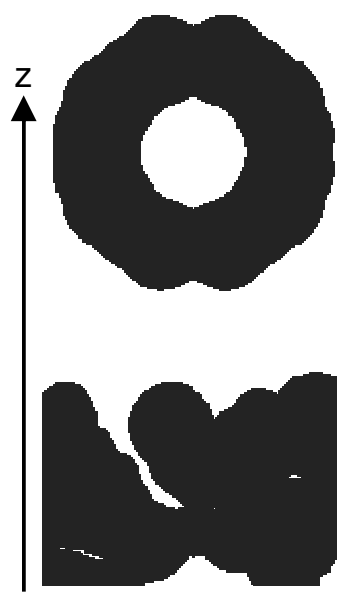

(b) $d=4.41 \AA$
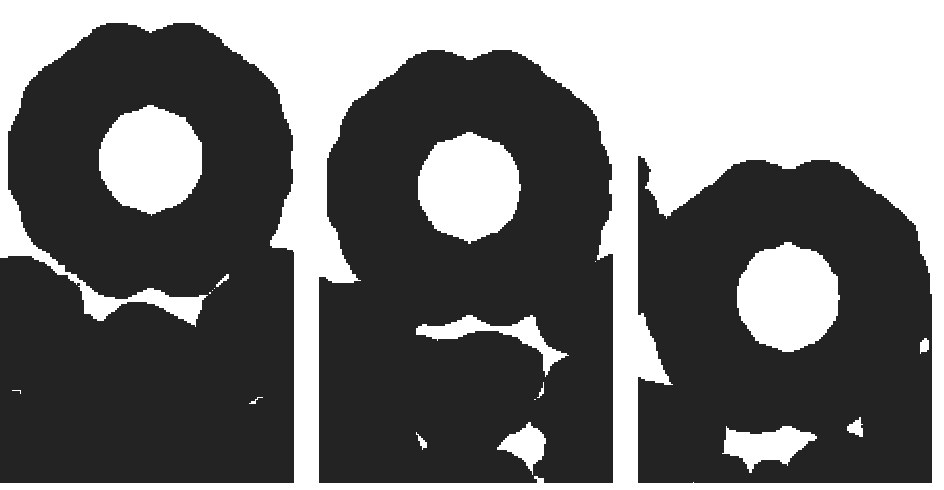

Figure 7.5 Overlayed snapshots of vdW contours of the unmodified polyester surface and $\mathrm{C}_{60}$ particle during indentation.

The features of the loading curve for unmodified polyester (Polyester- $\mathrm{C}_{60}$ ) presented in Figure 7.4, match qualitative features of experimental loading tests, ${ }^{211}$ albeit on different scales of force and separation. Specifically, starting at a large separation, the force $F_{z}$ is negative and the particle is attracted towards the Polyester. During the initial attraction, we observe two distinct features in the shape of the plot. The first feature is an increase in the magnitude of the attractive force between $\mathrm{C}_{60}$ and Polyester at separations of 9.0 to $4.6 \AA$. This increase in the strength of attraction is accompanied by a 'jump to contact' with $\mathrm{C}_{60}$ as shown by the change in structure from Figure 7.5a-b. This 'jump to contact' is driven by the tendency of the atoms near the surface of Polyester to bulge up slightly to meet the particle, which is clear from the relative movement of the surface in the $\mathrm{z}$ direction. The second feature noted during the stage of attractive interaction between $\mathrm{C}_{60}$ and Polyester, is a reduction in the strength of attractive force at separation from 4.6 to $3.1 \AA$. Here, surface atoms that are in very close proximity to the carbon particle repel it while the underlying polymer atoms continue to attract the carbon particle. The net effect is a reduced strength of attractive force between particle and surface. This force response is accompanied by compression and rearrangement of protruding atoms as a surface cavity is formed (Figure 7.5b-c). This is in agreement with the work of Henry et al. ${ }^{98}$ which has shown that due to the strong adhesion between $\mathrm{C}_{60}$ and polyester, fullerenes are able to partially infiltrate the flexible polymer by opening 
up surface cavities. Upon further advancement of the carbon particle into the surface, the sign of the force $F_{z}$ becomes positive and the particle is repelled from the Polyester model. At a separation interval between 3.1 to $1.5 \AA$, we observe a monotonic rise in the magnitude of the repulsive force between $\mathrm{C}_{60}$ and Polyester resulting from indentation of the underlying layer (Figure 7.5d). The gradient of this force rise from the loading curve of Figure 7.4, provides a measure of the level of surface hardness of the coating, and the steeper the gradient, the harder the surface. At very small separations the strength of the repulsive force begins to vary in a non-monotonic fashion due to plastic deformation and further envelopment of the particle by the surface.

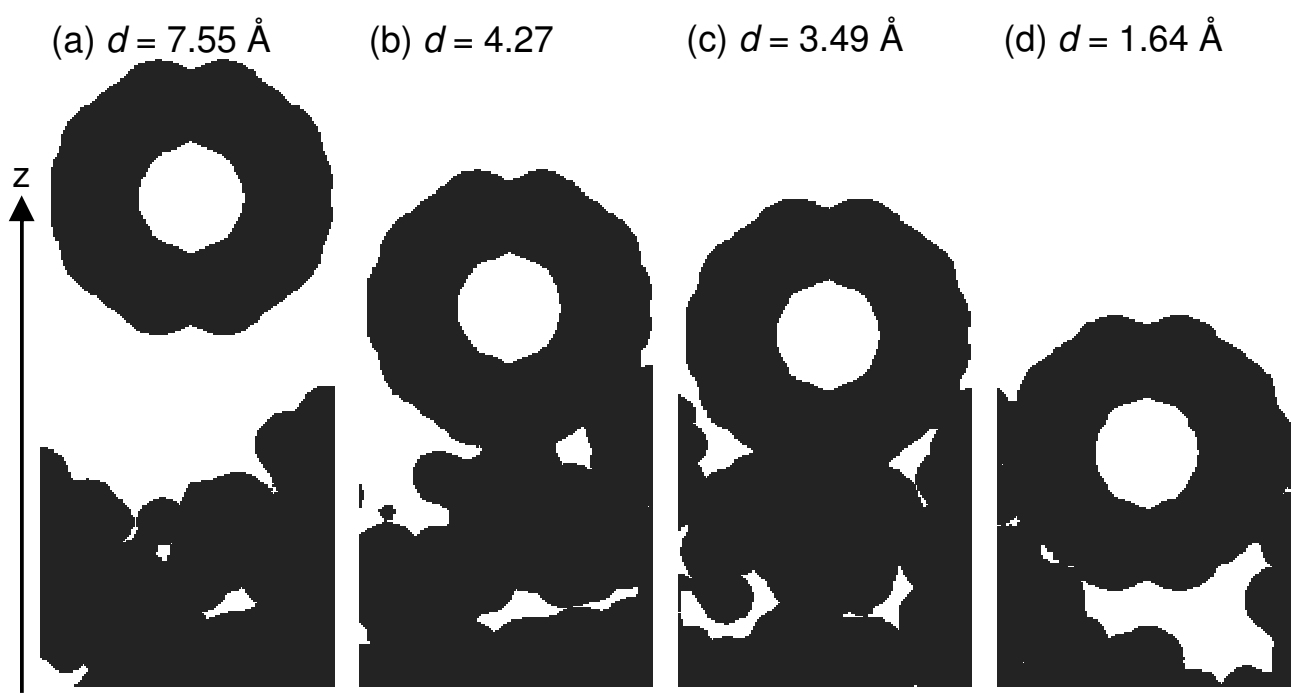

Figure 7.6 Overlayed snapshots of vdW contours of the Polyester15HDI surface and $\mathrm{C}_{60}$ particle during indentation.

The loading curve of hexamethylene crosslinked polyester (Polyester15HDI-C 60 ), exhibits features different from those detected in the loading curve of the unmodified polymer (Figure 7.4). The first such feature is a reduced strength of attractive force between $\mathrm{C}_{60}$ and Polyester15HDI at separations larger than $4.2 \AA$. This suggests a weaker long range adhesion between $\mathrm{C}_{60}$ and the crosslinked surface than between $\mathrm{C}_{60}$ and the untreated surface. The second characteristic feature occurs at a separation of 4.8 $\AA$ to $2.9 \AA$ and consists of an increase in the strength of the attractive force followed by a plateau-like response in $F_{z}$, not observed in the loading curve of the unmodified polyester. As a result of this, at separations of less than $4.2 \AA$, the $\mathrm{C}_{60}$ exhibits a stronger attraction with the hexamethylene crosslinked surface than with the original polyester 
layer. Inspection of the hexamethylene polyester model reveals that the protruding HDI crosslinks exhibit a relatively large displacement towards the $\mathrm{C}_{60}$ particle during the jump to contact (Figure 7.6a-b in the previous page). This displacement results from the increase in the strength of the attractive force between $\mathrm{C}_{60}$ and Polyester15HDI. In addition to this, Figure 7.6c shows that at a separation of $3.49 \AA$, protruding HDI moieties can envelope the particle by wrapping around its periphery. Such an early envelopment is not observed in the case of the original untreated polyester (Figure 7.5c), where the surface protrusions are less pronounced. Overall, it appears that the HDI crosslinks make the surface more deformable initially (upon first contact with $\mathrm{C}_{60}$ ) than the untreated polyester. However, in response to indentation of the crosslinked surface, we observe a steeper rise in the force $F_{z}$ compared to the response of the untreated polymer. This indicates an overall increase in hardness of the crosslinked film, despite the fact that the protruding HDI crosslinks are more deformable than the untreated polyester.

The loading curve of the isophorone crosslinked polyester also exhibits features which differ from those identified in the force plot of the original polymer. In particular, we observe a smaller initial rise in the attractive force, indicating a weaker long range attraction between $\mathrm{C}_{60}$ and the isophorone polyester than between $\mathrm{C}_{60}$ and the untreated polyester. In addition to this, the loading curve of isophorone polyester exhibits a distinct saw-tooth like variation in the force at separations of $\sim 4.8$ to $2.7 \AA$. These abrupt force fluctuations can be caused by protruding IPDI crosslinks that can be displaced by the $\mathrm{C}_{60}$ during loading. An example of this is illustrated in the following page Figure 7.7, which depicts characteristic conformations of both crosslinked systems during loading. The images highlight differences in the restructuring of the interfaces. The first type of restructuring is commonly observed for the hexamethylene crosslinked polymer. Protruding HDI moieties adjust conformationally by matching the contour of the carbon particle (Figure 7.7a). The result is an envelopment of the particle by the surface as shown from Figure 7.6b-c. Similar envelopment has also been observed in the case of the unmodified polyester. 
(a) Polyester15HDI-C $60 \stackrel{\mathbf{z}}{\leftarrow} \mathbf{x}$

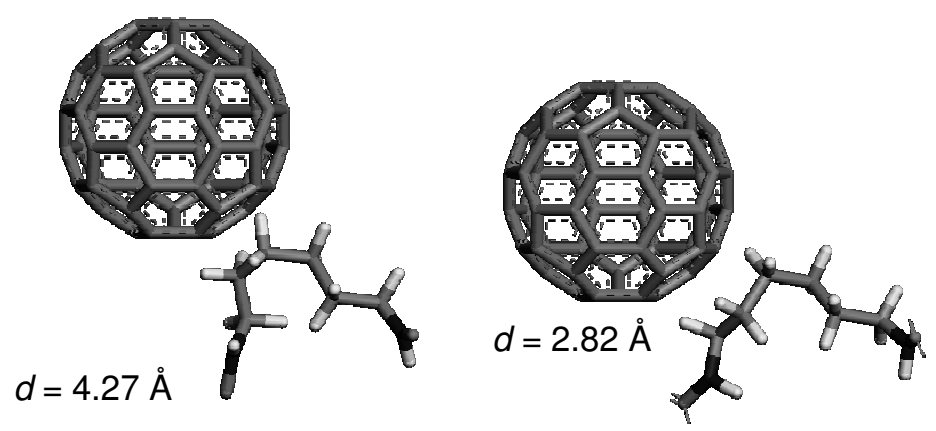

(b) Polyester14IPDI-C $60 \stackrel{\mathbf{z}}{\longleftrightarrow} \mathbf{y}$

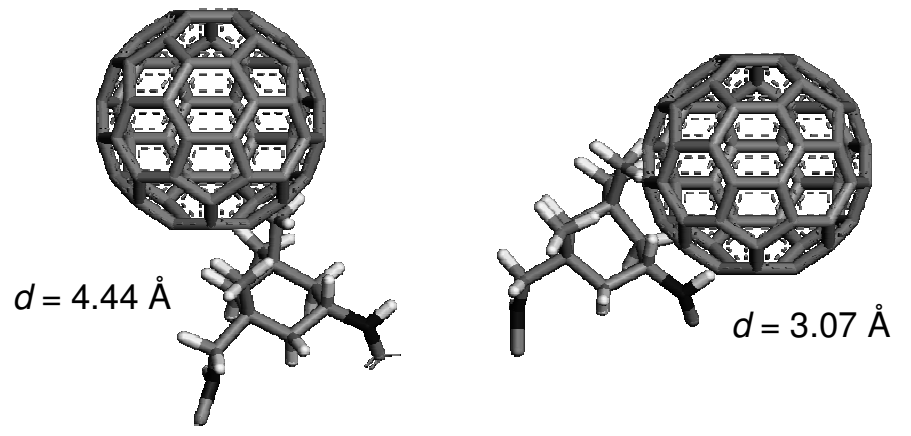

Figure 7.7 Snapshots of the equilibrated structures during loading highlighting two common forms of interfacial re-structuring: (a) conformational change of hexamethylene di-isocyanate (b) displacement of isophorone di-isocyanate.

The second type of restructuring is commonly observed for the isophorone crosslinked polymer. Here, the IPDI molecules rather than change conformations move out of position due to the stress imposed by the particle on the surface, causing abrupt fluctuation in the force $F_{z}$. Such force fluctuations are often a characteristic feature of stick-slip phenomena, occurring when the coefficient of static friction, the force needed to initiate movement, is higher than the coefficient of kinetic friction, the force during movement. This prompts an intermittent motion and an abrupt force response rather than a smooth force response. On the atomic-scale, this stick-slip phenomenon is expected to be particularly prevalent for hard and atomically rough surfaces which often would exhibit high coefficient of static friction. For example, in the experimental work of Rao et $\mathrm{al},{ }^{212}$ polymer surfaces were irradiated with $\mathrm{Ar}^{+}$ions, and the friction coefficient of the samples was monitored as a steel sphere was traversed along the surfaces using a 
friction microprobe. A wave-like friction response was noted for the irradiated films. This response was attributed to the crosslinked structure of the treated samples, and believed to be caused by periodic extension and sudden release of the surface moieties during adhesion with the steel sphere. The magnitude of the attractive force between $\mathrm{C}_{60}$ and isophorone polymer remains smaller than that noted between $\mathrm{C}_{60}$ and the untreated sample, even when the particle is brought close to the surface ( $4 \AA)$. In addition to this, the extent of contact between $\mathrm{C}_{60}$ and Polyester14IPDI (Figure 7.8a-b) is notably smaller than the contact between $\mathrm{C}_{60}$ and the untreated polymer (Figure 7.5b-c). This behavior is in contrast to that observed for the hexamethylene crosslinked polyester, which exhibits a much stronger short range attraction with $\mathrm{C}_{60}$, leading to the envelopment by the deformable HDI species (Figure 7.6c). Lastly, the repulsion associated with indentation of the isophorone crosslinked polymer increases more rapidly than the corresponding repulsive force rise of the untreated film. This suggests an increase in surface hardness as a result of IPDI crosslinking. However, at very small separations and large loads, the strength of repulsive force fluctuates considerably pointing to significant deformation of the isophorone crosslinked layer.

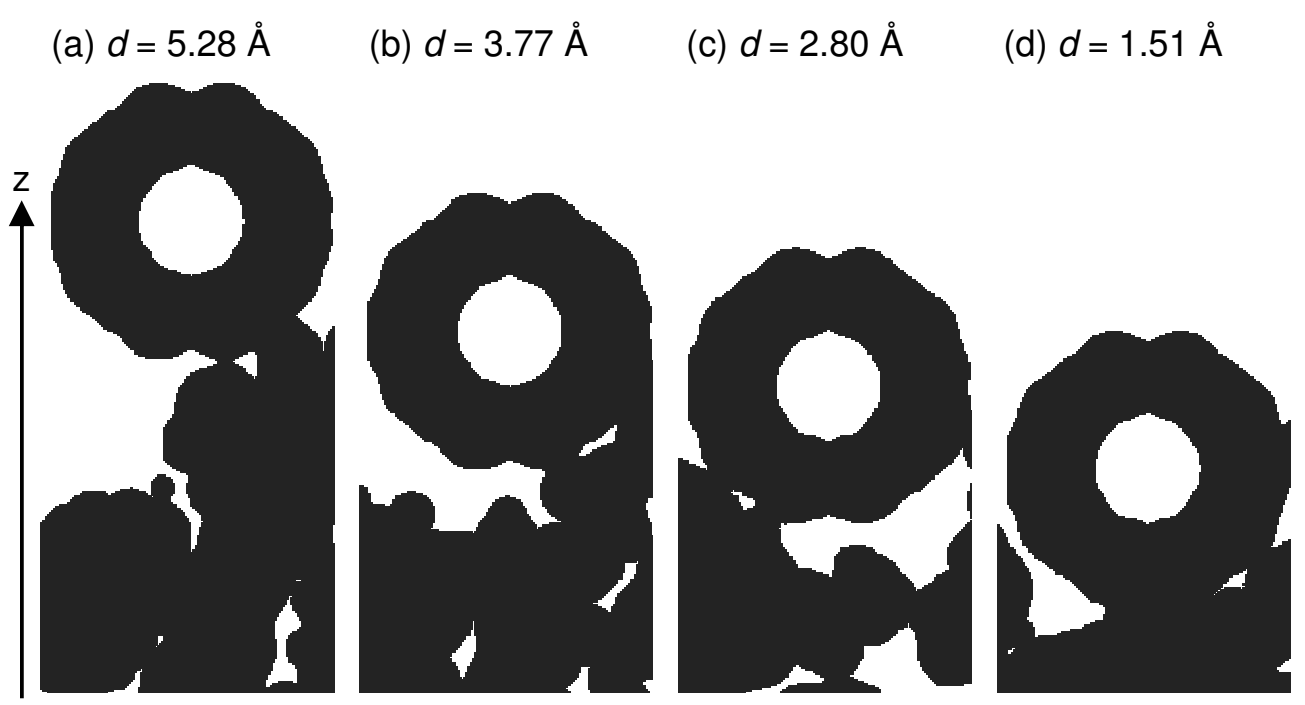

Figure 7.8 Overlayed snapshots of vdW contours of the Polyester14IPDI surface and $\mathrm{C}_{60}$ particle during indentation. 


\subsubsection{Adhesion plots of polyester- $\mathrm{C}_{60}$ systems}

The adhesion energy between fullerene and each polyester sample was evaluated by integrating the force $F_{z}$ during loading of the surfaces. Plots of adhesion energy versus separation $d$ are shown in Figure 7.9 from which the values of the work of adhesion and equilibrium separation were determined. The work of adhesion between unmodified polyester (Polyester) and $\mathrm{C}_{60}$ is $24.74 \mathrm{~mJ} \mathrm{~m}^{-2}$. This is in good agreement with the experimental work of Lukey et al. ${ }^{213}$ who used the Johnson-Kendall-Roberts (JKR) theory to calculate the work of adhesion between micron-sized carbon spheres and polyester and found it to be $34 \mathrm{~mJ} \mathrm{~m}^{-2}$. We also observe that our value for the work of adhesion is significantly less than the work of separation $\left(W_{\text {sep }}\right)$ value previously predicted by molecular dynamics simulations of fullerene and polyester interfaces $(79.4$ $\left.\mathrm{mJ} \mathrm{m}^{-2}\right){ }^{98}$ This difference is to be expected since, the work of adhesion takes into account additional energy due to diffusion, surface segregation, relaxation, and/or reconstruction during cleavage of the interface.

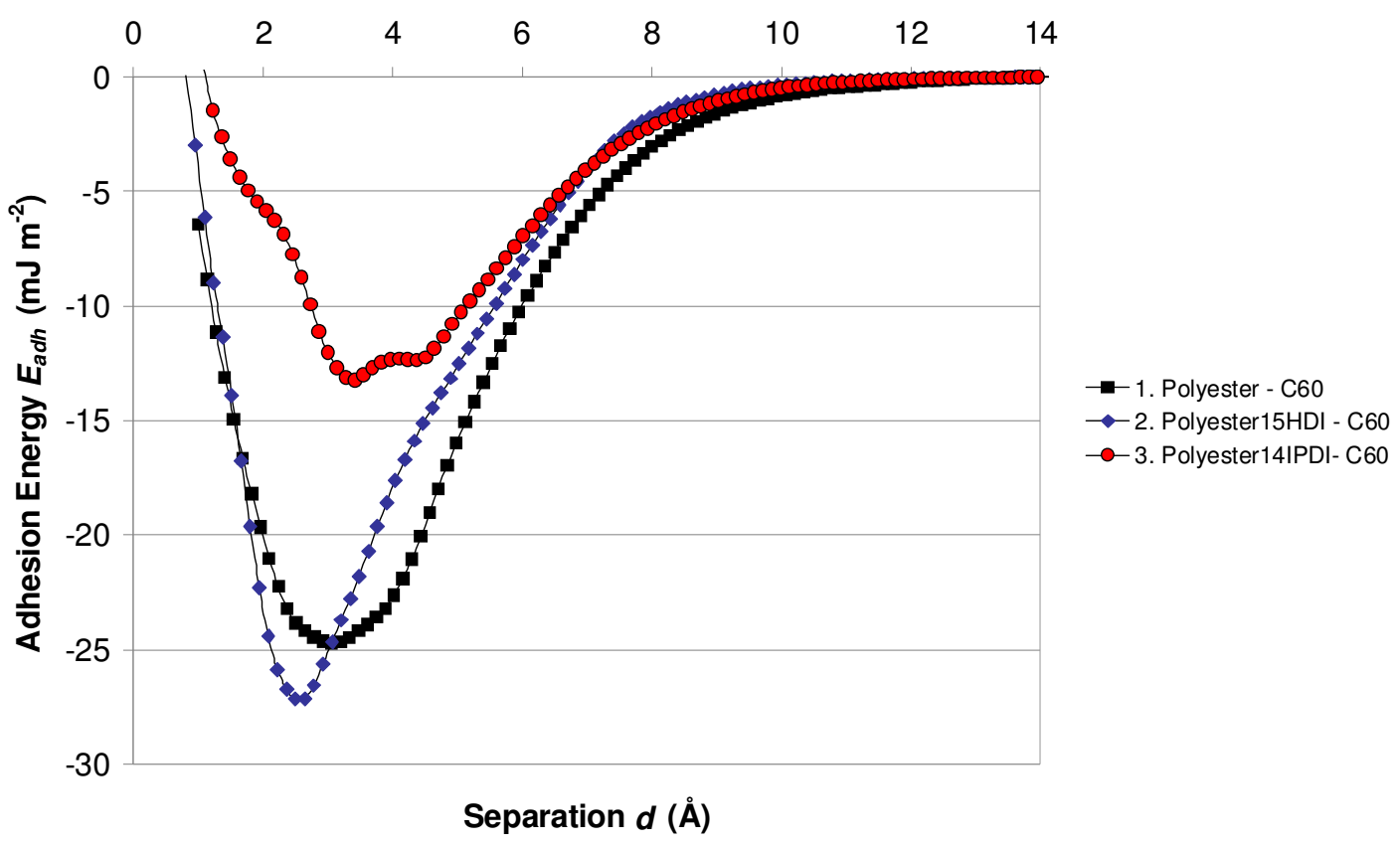

Figure 7.9 Adhesion energy plots for polyester- $\mathrm{C}_{60}$ systems

From Figure 7.9, the work of adhesion between $\mathrm{C}_{60}$ and hexamethylene crosslinked polyester is found to be $27.17 \mathrm{~mJ} \mathrm{~m}^{-2}$ and $\sim 10 \%$ stronger in magnitude than that for 
untreated polyester. Also, the equilibrium separation $\left(d_{0}\right)$ between fullerene and hexamethylene crosslinked polyester is $\sim 0.6 \AA$ smaller than the $d_{0}$ value of the untreated polyester-fullerene interface. Clearly, surface crosslinking with hexamethylene diisocyanate molecules results in a stronger adhesion between polymer and fullerene. A possible explanation for this behaviour is associated with the protruding deformable hexamethylene residues. These residues wrap and / or move around the particle's periphery as shown in Figure 7.6c, enveloping the particle and increasing its adhesion to the surface.

In the case of the isophorone crosslinked polyester, the work of adhesion between the surface and $\mathrm{C}_{60}$ is $13.23 \mathrm{~mJ} \mathrm{~m}^{-2}$ and $\sim 47 \%$ weaker in magnitude compared to unmodified polyester. The isophorone crosslinked polymer also exhibits a $\sim 0.4 \AA$ increase in its equilibrium separation $\left(d_{0}\right)$ with fullerene. Therefore, in contrast to the hexamethylene crosslinked layer, surface crosslinking of polyester using isophorone diisocyanates leads to a significant reduction in adhesion with fullerene. This behaviour can be associated with the observed reduction in surface deformation and interfacial restructuring of the IPDI residues. Isophorone di-isocyanate molecules have a higher molecular weight than their hexamethylene counterparts (see Figure 7.1) and also, due to the presence of a ring in the IPDI structure, can be expected to exhibit reduced deformability, ${ }^{198}$ elasticity $^{214}$ and thermal expansion. ${ }^{204}$ Experimental studies have shown that reduced interfacial restructuring can result in a lowered interfacial adhesion. Kinning $^{215}$ formulated silicone-polyurea copolymers containing poly(dimethylsiloxane) (PDMS) with varying amounts of soft segments (polypropylene oxide diamine) as well as hard segments (isophorone di-isocyanate and 1,3-diamino pentane groups). Adhesion strength between each co-polymer and an alkyl acrylate based adhesive tape were measured. It was suggested that low adhesion can be more readily attained for the copolymers having a higher hard segment content, due to their reduced segmental mobility and reduced degree of interfacial restructuring. In the experimental study of Lukey et al. $^{213}$ it was shown that work of adhesion between polyester and carbon decreases with increasing crosslink density. The crosslinking molecule studied was hexamethoxymethylmelamine (HMMM). It exhibits a similar cyclic structure to isophorone di-isocyanate. It is believed that for samples with a high HMMM content, the 
crosslinks segregate to the upper layers of the samples. ${ }^{201}$ This effectively reduces the extent of deformation of the surface region of the samples and in turn results in a reduced contact and adhesion between particle and surface. These experimental findings are in qualitative agreement with our observation of the simulated adhesion / mechanisms of crosslinked surfaces of varying deformability.

While the hexamethylene and isophorone crosslinked polymers exhibit a considerably different strength of adhesion with fullerene, these crosslinked polymers share one notably common trend. Adhesion plots of Figure 7.9 show that at separations larger than $\sim 4 \AA$, both crosslinked polyesters exhibit a weaker long range adhesion with $\mathrm{C}_{60}$ compared to unmodified polyester. Our calculated values of adhesion energy $\left(E_{a d h}\right)$ are directly affected by the vdW interactions of the system and not by electrostatic interaction since constituent atoms of fullerene carry a zero partial atomic charge. Therefore variations in adhesion can in theory be explained by the Hamaker relation, characterising the vdW interaction energy $(W)$ between macroscopic pairs of bodies of fixed geometries, in this case, a spherical particle and an infinite surface (see eq. (4.4)).

According to the Hamaker relation, a reduction in the atomic density of the surface can lessen the overall vdW interaction energy. We have shown that on completion of the surface crosslinking process, the crosslinker fragments protrude from the polyester reducing its overall atomic density i.e., the protruding fragments create a zone of lowered surface density. This reduction in atomic density is a possible cause for the weaker long range adhesion we have observed between fullerene and surface crosslinked polymers. However, as the carbon particle is brought closer towards the polymers, the surfaces are seen to deform as protruding segments of the polymer jump into contact with $\mathrm{C}_{60}$, and cavity formation in the surface is observed. This surface restructuring perturbs the fixed geometries assumed by the Hamaker relation. ${ }^{13}$ For this reason the atomic density alone is not sufficient to accurately describe all trends in interfacial adhesion, especially those at short interfacial separations. This observation is exemplified in the case of the hexamethylene crosslinked polyester where surface restructuring appears to be particularly prevalent. Evidently, the hexamethylene crosslinked polyester exhibits a weaker long-range adhesion with fullerene compared to unmodified polyester, whereas at shorter separations, the reverse is true i.e., the 
hexamethylene crosslinked polyester exhibits a significantly stronger work of adhesion with $\mathrm{C}_{60}$ than unmodified polyester.

\subsubsection{Hardness of polyester surfaces}

To be able to quantify the hardness of the outermost and sub-surface regions of the coatings, we determined the hardness of the polymers at two separate force values of 0.3 $\mathrm{nN}$ and $0.6 \mathrm{nN}$, respectively. Hardness measurements undertaken at $0.3 \mathrm{nN}$ resulted in very small indentation depths (less than $1 \AA$ ) and therefore represent the average hardness of the topmost region of the surface. In comparison, hardness measurements undertaken at $0.6 \mathrm{nN}$ resulted in larger indentation depths (greater than $1 \AA$ ) and represent the average hardness of the sub-surface region of the coating.

Table 7.1 Hardness Parameters for Polyester Surfaces

\begin{tabular}{cccc}
\hline \multirow{2}{*}{ Force $(\mathrm{nN})$} & \multicolumn{3}{c}{ Surface Hardness (MPa) } \\
& Polyester & Polyester15HDI & Polyester14IPDI \\
\hline 0.30 & 192.19 & 207.19 & 277.69 \\
0.60 & 264.13 & 370.50 & 280.45
\end{tabular}

In the case of the original polyester surface, the hardness value measured at $0.3 \mathrm{nN}$ is $\sim 27 \%$ smaller than that measured at $0.6 \mathrm{nN}$. This is to be expected, since residues at the topmost region of the surface exhibit greater mobility than those located closer towards the core of the layer. Typical hardness values of polyester films such as polyethylene-terepthalate (PET) lie in the range of $100 \mathrm{MPa}$ to $300 \mathrm{MPa}$ and depend on properties such as the level of crosslinking and or crystallinity. For example, Fakirov et al. $^{216}$ determined the hardness of amorphous uncrosslinked polyester to be $120 \mathrm{MPa}$, while Frings et al. ${ }^{217}$ predicted the average hardness of amorphous crosslinked polyester to be $240 \mathrm{MPa}$. More recently, Beake et al. ${ }^{218}$ determined the average hardness of a semi-crystalline PET film to be 250MPa. Our predicted hardness measurements of the untreated polyester model are slightly higher than those obtained by experimental indentation tests of amorphous uncrosslinked polyester, since all our models are bulk 
crosslinked. Our values do however agree well with experimental hardness measurements of semi-crystalline or crosslinked polyester films.

The value of surface hardness evaluated at $0.3 \mathrm{nN}$ for the hexamethylene crosslinked polymer is slightly greater $(\sim 8 \%)$ than the equivalent value for the untreated polyester. However, indented at $0.6 \mathrm{nN}$, the hexamethylene polyester is significantly harder $(\sim 40 \%)$ that the untreated model. Therefore, surface crosslinking of polyester with hexamethylene di-isocyanate molecules leads to an increase in indentation hardness at surface depths greater than $\sim 1 \AA$, but the topmost region of the crosslinked surface still maintains significant mobility / deformability.

In contrast, isophorone crosslinked polyester exhibits a significant increase in surface hardness measured at $0.3 \mathrm{nN}$ relative to the untreated polymer ( $44 \%)$, but only a small increase in hardness evaluated at $0.6 \mathrm{nN}(\sim 6 \%)$. Therefore, surface crosslinking of polyester with isophorone di-isocyanate molecules is predicted to result in a significant increase in surface hardness at the topmost region of the layer (at indentation depths less than $1 \AA$ ) while only a slight increase in hardness is expected at greater depths into the surface. In comparison to the hexamethylene di-isocyanate surface crosslinks, the isophorone molecules appear to have a more localised effect on surface rigidity.

\subsection{Conclusion}

In this section, we presented a methodology to atomistically simulate chemical crosslinking of polymers. In conjunction with this, using molecular mechanics and dynamics, we simulated a nanoindentation process whereby nano-particles (fullerene in this case) are lowered in step wise manner onto different regions of the polymer surface enabling us to simultaneously quantitatively characterise the average surface hardness of the polymer film and calculate the work of adhesion between polymer and indenter particle. Our results indicate that polyester surface crosslinking with hexamethylene diisocyanate molecules leads to an increase in indentation hardness at surface depths greater than $\sim 1 \AA$, but the top surface of this crosslinked layer appears to maintain significant mobility. The hexamethylene residues have a linear structure, and those that protrude from the surface are deformable, resulting in a stronger adhesion between 
surface and fullerene compared to the untreated polymer. In contrast, isophorone surface crosslinks result in significantly weaker adhesion with carbon, due to their less deformable rigid structure. In this case, rigidity is imparted to the polymer top surface, whilst at the same time the flexibility of the core region of the polymer is maintained. 


\section{Summary}

The leaf of the Lotus plant provides an elegant example of how a natural surface can remain clean even in the dirtiest of environments. The leaf's fine-scale surface structure combined with its hydrophobic chemistry ensures water droplets bead off its surface, carrying away contaminant particles. An alternative dirt-shedding surface, also found in nature, is based on hydrophilic surface chemistry. Water droplets that come into contact with hydrophilic surfaces spread, forming a thin film of water lifting contaminants from the surface. There is significant motivation to pursue both of these types of behaviour for man made systems because of the wide range of possible applications ranging from selfcleaning paints to dirt-resistant clothing. However, for some applications, the synthesis of a self-cleaning surface is indeed a difficult task to achieve, and one needs to first elucidate the precise properties required to generate a certain level of resistance to the adhering contaminant. Atomistic simulations provide a useful tool to gain such insight and help design surfaces where stay-clean properties are highly desirable. Here, using force-field based molecular mechanics and dynamics we explored the properties of polymer surfaces in order to develop design aspects for self-cleaning industrial paintcoatings. A polyester surface model was constructed, based on a realistic cured paint coating while various carbon models were selected, to emulate commonly encountered atmospheric dirt particulates. A fundamental understanding of the nature of interactions between contaminant and polymer coating in various environments, including aqueous conditions, was achieved and nanoscale modifications of the coating that help reduce the strength of the adhering contaminant were explored. The modifications were based on hydrophobic or alternatively hydrophilic surface treatments. We demonstrated that the chemistry, morphology and stability of the surface play a vital role in resisting adhesion of contaminant particles. Nanoscale surface modification combined with fine-scale roughness reduced adhesion between coating and contaminant by up to $21 \%$. The newly formed surface functional groups comprise of 'heavy' atoms which repel the carbon contaminant at a close proximity to the surface via vdW interactions. Meanwhile we showed that the atomic-scale surface roughness reduces the effective contact area between surface and contaminant, in accordance with the Cassie-Baxter construction. 
However, our modelling suggested that flexible polymer surfaces undergo significant rearrangement, even at ambient conditions, in agreement with aging and hydrophobic recovery studies. As a result of this, the physical and chemical properties of the coating that initially help shield the surface from the adhering contaminant are diminished with time. Even in aqueous environment, the mobility of the polymer chains plays a vital role in the long-term functionality of the surface. We proposed a surface crosslinking procedure, aimed at improving the hardness of the outer-region of the coating as a preventative measure against aging. For surface crosslinking of the polymer with isophorone di-isocyanate (IPDI) molecules, we observed a much improved stability of the coating's outer surface, and consequently a $47 \%$ weaker adhesion with our contaminant particle. The rigid outer surface prevents the polymer chains from wrapping around the contaminant, improving the coating's dirt-resistance capabilities. We anticipate that our modelling studies will be a starting point for the fabrication of a polymer coating that exhibits permanent dirt-shielding qualities. The synthesis of such a coating will require careful control of the chemistry, atomic-scale roughness, and stability of the surface. 


\section{Future Work}

We showed that similar to the natural stay-clean ability of plant leaves, the physical as well as chemical aspects of the material surface are critical in rendering a polymer coating self-cleaning. But we also showed that maintaining these properties over long periods of time remains a difficult task to achieve. This is particularly the case for naturally flexible polymers that are often associated with industrial polymer coatings. Future work may therefore include the design of a robust self-cleaning polymer surface that is resistant to aging effects or hydrophobic recovery. In this thesis, we have demonstrated that the surface crosslinking of polyester can improve the stability of the outer-most region of the material. In future work, this crosslinked surface model may therefore provide guidance to achieve stable attachment points for hydrophobic or hydrophilic functional groups. Furthermore, the surface crosslinking and in-silico nanoindentation procedures that were introduced here may be used as a starting point for the development and testing of an array of surface crosslinked polymer coatings.

An alternative approach to the design of stay-clean polymer coatings may be the development of a responsive surface requiring an external trigger rather than a selfcontained solution. Synthetic polymers capable of responses to external stimuli represent an exciting and emerging area in polymer science. There are a number of opportunities in the design, modelling and synthesis of stimuli-responsive surfaces, prompted by their endless scope of commercial applications. In regards to the stay-clean properties of polymer coatings, a stimuli-responsive mechanism can in theory induce morphological or chemical changes to the surface that could help retain its contamination-resistant properties over long periods of time, and in varying environmental conditions. However, there are significant challenges associated with having to maintain the mechanical integrity of the coating. There are a number of stimuli which can induce switching such as light, temperature, $\mathrm{pH}$ and solvent, however, not all of these mechanisms are appropriate for application to industrial coatings. Environmental triggers, such as light or temperature may provide the most attractive pathway for adaptable industrial paint coatings, but are harder to control and weaker compared to artificial triggers. 


\section{References}

(1) Weiss, K. D. Prog. Polym. Sci. 1997, 22, 203.

(2) Gauntt, D. L.; Clark, K. G.; Hirst, D. J.; Hegedus, C. R. J. Coating. Technol. 1991, 63, 25.

(3) Posfai, M.; Gelencser, A.; Simonics, R.; Arato, K.; Li, J.; Hobbs, P. V.; Buseck, P. R. J Geophys Res-Atmos 2004, 109, D06213.

(4) Parkin, I. P.; Palgrave, R. G. J. Mater. Chem. 2005, 15, 1689.

(5) Artus, G. R. J.; Jung, S.; Zimmermann, J.; Gautschi, H. P.; Marquardt, K.; Seeger, S. Adv. Mater. 2006, 18, 2758.

(6) Solga, A.; Cerman, Z.; Striffler, B. F.; Spaeth, M.; Barthlott, W. Bioinspir. Biomim. 2007, 2, S126.

(7) Barthlott, W.; Neinhuis, C. Planta 1997, 202, 1.

(8) Patankar, N. A. Langmuir 2004, 20, 8209.

(9) Neinhuis, C.; Barthlott, W. Ann. Bot.-London 1997, 79, 667.

(10) Mills, A.; Lepre, A.; Elliott, N.; Bhopal, S.; Parkin, I. P.; O'Neill, S. A. J. Photoch. Photobio. A 2003, 160, 213.

(11) Wang, R.; Hashimoto, K.; Fujishima, A.; Chikuni, M.; Kojima, E.; Kitamura, A.; Shimohigoshi, M.; Watanabe, T. Nature 1997, 388, 431.

(12) Young, T. Philos. T. R. Soc. Lond. 1805, 95, 65.

(13) Israelachvili, J. Intermolecular and Surface Forces; Academic Press: London, UK, 1992.

(14) Omenyi, S. N.; Neumann, A. W.; Vanoss, C. J. J. Appl. Phys. 1981, 52, 789.

(15) Owens, D. K.; Wendt, R. C. J. Appl. Polym. Sci. 1969, 13, 1741.

(16) Dettre, R. H.; Johnson, R. E. J. Colloid Interf. Sci. 1969, 31, 568.

(17) Grundke, K.; Augsburg, A. J. Adhes. Sci. Technol. 2000, 14, 765.

(18) Wu, S. J. Polym. Sci. Pol. Sym. 1971, 34, 19.

(19) Mittall, K. L. Polymer Surface Modification: Relevance to Adhesion; Brill Academic: Netherlands, 2000.

(20) Hata, T.; Kitazaki, Y.; Saito, T. J. Adhesion 1987, 21, 177.

(21) Wu, S. J. Phys. Chem.-Us. 1968, 72, 3332.

(22) Brandrup, J.; Immergut, E. H.; Grulke, E. A. Polymer Handbook; 4th ed.; John Wiley \& Sons: New York, 2003.

(23) Ratner, B. D.; Castner, D. G. Surface Modification of Polymeric Biomaterials; Plenum Press: New York, 1997.

(24) Janczuk, B.; Bialopiotrowicz, T. J. Colloid Interf. Sci. 1989, 127, 189.

(25) Kitazaki, Y.; Hata, T. J. Adhesion 1972, 4, 123.

(26) Boulangé-Petermann, L.; Debacq, C.; Poiret, P.; Cromières, B. In Contact Angle, Wettability and Adhesion; Mittal, K. L., Ed.; VPS: 2003; Vol. 3.

(27) Brandrup, J.; Immergut, E. H. Polymer Handbook; 3rd ed.; John Wiley \& Sons: New York, 1989.

(28) Ko, Y. C.; Ratner, B. D.; Hoffman, A. S. J. Colloid Interf. Sci. 1981, 82, 25.

(29) Caykara, T.; Yerlikaya, Z.; Kantoglu, O. J. Macromol. Sci. A 2003, 40, 1173. 
(30) Mittall, K. L. Adhesion Aspects Of Thin Films; Brill Academic: Netherlands, 2005.

(31) Starov, V. M.; Kosvintsev, S. R.; Velarde, M. G. J. Colloid Interf. Sci. 2000, 227, 185.

(32) Dupré, A. Theorie Mecanique de la Chaleur; Gauthier-Villars: Paris, 1869 .

(33) Jarvis, S. P.; Pethica, J. B. Thin Solid Films 1996, 273, 284.

(34) Binggeli, M.; Mate, C. M. Appl. Phys. Lett. 1994, 65, 415.

(35) Frisbie, C. D.; Rozsnyai, L. F.; Noy, A.; Wrighton, M. S.; Lieber, C. M. Science 1994, 265, 2071.

(36) Malshe, V. C.; Sangaj, N. S. Prog. Org. Coat. 2005, 53, 207.

(37) Montefusco, F.; Bongiovanni, R.; Priola, A.; Ameduri, B. Macromolecules 2004, 37, 9804.

(38) Sigurdsson, S.; Shishoo, R. J. Appl. Polym. Sci. 1997, 66, 1591.

(39) Selli, E.; Mazzone, G.; Oliva, C.; Martini, F.; Riccardi, C.; Barni, R.; Marcandalli, B.; Massafra, M. R. J. Mater. Chem. 2001, 11, 1985.

(40) Kim, Y.; Lee, Y.; Han, S.; Kim, K.-J. Surf Coat Tech 2006, 200, 4763.

(41) Wen, C.-H.; Chuang, M.-J.; Hsiue, G.-H. Thin Solid Films 2006, 503,

(42) Ghenaim, A.; Elachari, A.; Louati, M.; Caze, C. J. Appl. Polym. Sci. 2000, 75,10 .

(43) Hong, S. I.; Kim, K. B.; Lee, Y.; Cho, S. Y.; Ko, J. A.; Hong, S. K.; Park, H. J. J. Appl. Polym. Sci. 2009, 113, 2988.

(44) Tressaud, A.; Durand, E.; Labrugere, C.; Kharitonov, A. P.; Kharitonova, L. N. J. Fluorine Chem. 2007, 128, 378.

(45) Kharitonov, A. P. Prog. Org. Coat. 2008, 61, 192.

(46) Lee, S. A.; Oh, S. H.; Lee, W. J. Colloid Interf. Sci. 2009, 332, 461.

(47) Kharitonov, A. P. J. Fluorine Chem. 2000, 103, 123.

(48) Leroux, J. D.; Paul, D. R.; Arendt, M. F.; Yuan, Y.; Cabasso, I. J. Membrane. Sci. 1994, 90, 37.

(49) Vandencasteele, N.; Merche, D.; Reniers, F. Surf. Interface Anal. 2006, $38,526$.

(50) Vandencasteele, N.; Reniers, F. Surf. Interface Anal. 2004, 36, 1027.

(51) Song, W. L.; Veiga, D. D.; Custodio, C. A.; Mano, J. F. Adv. Mater. 2009, 21, 1830.

(52) Mizes, H. A.; Loh, K. G.; Miller, R. J. D.; Ahuja, S. K.; Grabowski, E. F. Appl. Phys. Lett. 1991, 59, 2901.

(53) Wenzel, R. N. Ind. Eng. Chem. 1936, 28, 988.

(54) Mardare, D.; Luca, D.; Teodorescu, C. M.; Macovei, D. Surf. Sci. 2007, $601,4515$.

(55) Jung, Y. C.; Bhushan, B. Nanotechnology 2006, 17, 4970.

(56) Coulson, S. R.; Woodward, I.; Badyal, J. P. S.; Brewer, S. A.; Willis, C. J. Phys. Chem. B 2000, 104, 8836.

(57) Cassie, A. B. D.; Baxter, S. T. Faraday Soc. 1944, 40, 0546.

(58) Yu, J. G.; Zhao, X. J.; Zhao, Q. N.; Wang, G. Mater. Chem. Phys. 2001, $68,253$. 
(60) Inoue, Y.; Yoshimura, Y.; Ikeda, Y.; Kohno, A. Colloid. Surface. B 2000, $19,257$.

(61) Marmur, A. Langmuir 2004, 20, 3517.

(62) Zhang, L.; Zhou, Z. L.; Cheng, B.; DeSimone, J. M.; Samulski, E. T. Langmuir 2006, 22, 8576.

(63) Ming, W.; Wu, D.; van Benthem, R.; de With, G. Nano Lett. 2005, 5, 2298.

(64) Garbassi, F.; Morra, M.; Occhiello, E. Polymer Surfaces- From Physics to Technology; Wiley: Chichester, 1994.

(65) Andrade, J. D.; Chen, W. Y. Surf. Interface Anal. 1986, 8, 253.

(66) Baszkin, A.; Terminas.L Polymer 1974, 15, 759.

(67) Holly, F. J. J. Biomed. Mater. Res. 1975, 9, 315.

(68) Zhang, H.; Lamb, R.; Lewis, J. Sci. Technol. Adv. Mat. 2005, 6, 236.

(69) Rios, P. F.; Dodiuk, H.; Kenig, S.; McCarthy, S.; Dotan, A. Polym. Advan. Technol. 2008, 19, 1684.

(70) Zimmermann, J.; Reifler, F. A.; Schrade, U.; Artus, G. R. J.; Seeger, S. Colloid. Surface. A 2007, 302, 234.

(71) Lee, S.; Kang, J. H.; Lee, S. J.; Hwang, W. Lab Chip 2009, 9, 2234.

(72) Houmard, M.; Riassetto, D.; Roussel, F.; Bourgeois, A.; Berthome, G.; Joud, J. C.; Langlet, M. Surf. Sci. 2008, 602, 3364.

(73) Sun, H. J. Phys. Chem. B 1998, 102, 7338.

(74) Misra, S.; Fleming, P. D.; Mattice, W. L. J. Comput.-Aided Mater. 1995, 2,101 .

(75) Natarajan, U.; Misra, S.; Mattice, W. L. Comput. Theor. Polym. S. 1998, $8,323$.

(76) Wu, C. F.; Xu, W. J. Polymer 2006, 47, 6004.

(77) Rappe, A. K.; Casewit, C. J.; Colwell, K. S.; Goddard, W. A.; Skiff, W. M. J. Am. Chem. Soc. 1992, 114, 10024.

(78) Mayo, S. L.; Olafson, B. D.; Goddard, W. A. J. Phys. Chem.-Us. 1990, $94,8897$.

(79) Cornell, W. D.; Cieplak, P.; Bayly, C. I.; Gould, I. R.; Merz, K. M.; Ferguson, D. M.; Spellmeyer, D. C.; Fox, T.; Caldwell, J. W.; Kollman, P. A. J. Am. Chem. Soc. 1996, 118, 2309.

(80) Mackerell, A. D.; Wiorkiewiczkuczera, J.; Karplus, M. J. Am. Chem. Soc. 1995, 117, 11946.

(81) Jorgensen, W. L.; Maxwell, D. S.; TiradoRives, J. J. Am. Chem. Soc. 1996, $118,11225$.

(82) Allinger, N. L.; Yuh, Y. H.; Lii, J. H. J. Am. Chem. Soc. 1989, 111, 8551.

(83) Lii, J. H.; Allinger, N. L. J. Am. Chem. Soc. 1989, 111, 8566.

(84) Lii, J. H.; Allinger, N. L. J. Am. Chem. Soc. 1989, 111, 8576.

(85) Allinger, N. L.; Chen, K. S.; Katzenellenbogen, J. A.; Wilson, S. R.; Anstead, G. M. J. Comput. Chem. 1996, 17, 747.

(86) Allinger, N. L.; Chen, K. S.; Lii, J. H. J. Comput. Chem. 1996, 17, 642.

(87) Nevins, N.; Chen, K. S.; Allinger, N. L. J. Comput. Chem. 1996, 17, 669. 
(88) Hwang, M. J.; Stockfisch, T. P.; Hagler, A. T. J. Am. Chem. Soc. 1994, 116,2515 .

(89) Maple, J. R.; Hwang, M. J.; Stockfisch, T. P.; Dinur, U.; Waldman, M.; Ewig, C. S.; Hagler, A. T. J. Comput. Chem. 1994, 15, 162.

(90) Maple, J. R.; Hwang, M. J.; Stockfisch, T. P.; Hagler, A. T. Israel. J. Chem. 1994, 34, 195.

(91) Todorova, N.; Legge, F. S.; Treutlein, H.; Yarovsky, I. J. Phys. Chem. B 2008, $112,11137$.

(92) Vergelati, C.; Perwuelz, A.; Vovelle, L.; Romero, M. A.; Holl, Y. Polymer 1994, 35, 262.

(93) Flory, P. J. Macromolecules 1974, 7, 381.

(94) Yarovsky, I.; Evans, E. Polymer 2002, 43, 963.

(95) Henry, D. J.; Lukey, C. A.; Evans, E.; Yarovsky, I. Mol. Simulat. 2005, $31,449$.

(96) Heine, D. R.; Grest, G. S.; Lorenz, C. D.; Tsige, M.; Stevens, M. J. Macromolecules 2004, 37, 3857.

(97) Henry, D. J.; Evans, E.; Yarovsky, I. Polymer 2007, 48, 2179.

(98) Henry, D. J.; Evans, E.; Yarovsky, I. J. Phys. Chem. B 2006, 110, 15963.

(99) Bermejo, J. S.; Ugarte, C. M. Macromol. Theor. Simul. 2009, 18, 317.

(100) Bermejo, J. S.; Ugarte, C. M. Macromol. Theor. Simul. 2009, 18, 259.

(101) Liu, J.; Cao, D.; Zhang, L. J. Chem. Phys. 2009, 131, 034903.

(102) Lin, P.-H.; Khare, R. Macromolecules 2009, 42, 4319.

(103) Varshney, V.; Patnaik, S. S.; Roy, A. K.; Farmer, B. L. Macromolecules 2008, $41,6837$.

(104) Komarov, P. V.; Yu-Tsung, C.; Shih-Ming, C.; Khalatur, P. G.; Reineker, P. Macromolecules 2007, 40, 8104.

(105) Fan, H. B.; Yuen, M. M. F. Polymer 2007, 48, 2174.

(106) Clancy, T. C.; Frankland, S. J. V.; Hinkley, J. A.; Gates, T. S. Polymer 2009, 50, 2736.

(107) Clancy, T. C.; Mattice, W. L. Comput. Theor. Polym. S. 1999, 9, 261.

(108) Yiapanis, G.; Henry, D. J.; Evans, E.; Yarovsky, I. J. Phys. Chem. C 2007, 111, 3000 .

(109) Mansfield, K. F.; Theodorou, D. N. Macromolecules 1991, 24, 4295.

(110) Attwood, D.; Marshall, P. I. Compos. Part a-Appl. S. 1996, 27A, 775.

(111) Williams, J. G.; James, M. R.; Morris, W. L. Composites 1994, 25, 757.

(112) Hocquet, A.; Langgard, M. J. Mol. Model. 1998, 4, 94.

(113) Liao, K.; Li, S. Appl. Phys. Lett. 2001, 79, 4225.

(114) Wong, M.; Paramsothy, M.; Xu, X. J.; Ren, Y.; Li, S.; Liao, K. Polymer 2003, 44, 7757.

(115) Yang, M. J.; Koutsos, V.; Zaiser, M. J. Phys. Chem. B 2005, 109, 10009.

(116) Henry, D. J.; Yiapanis, G.; Evans, E.; Yarovsky, I. J. Phys. Chem. B 2005, 109, 17224.

(117) Myers, D. Surfaces, Interfaces and Colloids: Principles and Applications; John Wiley \& Sons: Canada, 1999.

(118) Halgren, T. A. J. Am. Chem. Soc. 1992, 114, 7827. 
(119) Yarovsky, I.; Henry, D. J.; Yiapanis, G.; Evans, E. Chem. Aust. 2006, 73, 11.

(120) Jensen, F. Introduction to Computational Chemistry; John Wiley \& Sons: Denmark, 2007.

(121) Leach, A. R. Molecular Modelling: Principles and Applications; Longman, 1996.

(122) Werder, T.; Walther, J. H.; Jaffe, R. L.; Halicioglu, T.; Noca, F.; Koumoutsakos, P. Nano Lett. 2001, 1, 697.

(123) Fan, C. F.; Cagin, T. J. Chem. Phys. 1995, 103, 9053.

(124) Kisin, S.; Vukic, J. B.; van der Varst, P. G. T.; de With, G.; Konin, C. E. Chem. Mater. 2007, 19, 903.

(125) Swope, W. C.; Andersen, H. C.; Berens, P. H.; Wilson, K. R. J. Chem. Phys. 1982, 76, 637.

(126) Verlet, L. Phys. Rev. 1967, 159, 98.

(127) Karasawa, N.; Goddard, W. A. J. Phys. Chem.-Us. 1989, 93, 7320.

(128) Hill, T. L. An Introduction to Statistical Thermodynamics; Dover Publication, Inc.: New York, 1986.

(129) Woodcock, L. V. Chem. Phys. Lett. 1971, 10, 257.

(130) Berendsen, H. J. C.; Postma, J. P. M.; Vangunsteren, W. F.; Dinola, A.; Haak, J. R. J. Chem. Phys. 1984, 81, 3684.

(131) Andersen, H. C. J. Chem. Phys. 1980, 72, 2384.

(132) Haack, L. P.; Straccia, A. M.; Holubka, J. W.; Bhurke, A.; Xie, M.; Drzal, L. T. Surf. Interface Anal. 2000, 29, 829.

(133) Davidson, M. R.; Mitchell, S. A.; Bradley, R. H. Surf. Sci. 2005, 581, 169.

(134) Lee, M.; Lee, M. S.; Wakida, T.; Tokuyama, T.; Inoue, G.; Ishida, S.; Itazu, T.; Miyaji, Y. J. Appl. Polym. Sci. 2006, 100, 1344.

(135) Ghzaoui, E.; Lindheimer, M.; Lindheimer, A.; Lagerge, S.; Partyka, S. Colloid. Surface. A 2004, 233, 79.

(136) Ishiguro, T.; Takatori, Y.; Akihama, K. Combust. Flame 1997, 108, 231.

(137) Wentzel, M.; Gorzawski, H.; Naumann, K. H.; Saathoff, H.; Weinbruch, S. J. Aerosol Sci. 2003, 34, 1347.

(138) McKie, D.; McKie, C. Essentials of crystallography; Blackwell Scientific Publications: Oxford, 1986.

(139) Petersen, T.; Yarovsky, I.; Snook, I.; McCulloch, D. G.; Opletal, G. Carbon 2004, 42, 2457.

(140) Opletal, G.; Petersen, T.; O'Malley, B.; Snook, I.; McCulloch, D. G.; Marks, N. A.; Yarovsky, I. Mol. Simulat. 2002, 28, 927.

(141) Petersen, T.; Yarovsky, I.; Snook, I.; McCulloch, D. G.; Opletal, G. Carbon 2003, 41, 2403.

(142) Yarovsky, I. Aust. J. Phys. 1997, 50, 407.

(143) Finnis, M. W. J. Phys.-Condens. Mat. 1996, 8, 5811.

(144) Connolly, M. L. Science 1983, 221, 709.

(145) Reeves, W. A.; Beninate, J. V.; Perkins, R. M.; Drake, G. L. Am. Dyest. Rep. 1968, 57, 1053. 
(146) Allman, W. T.; Dunlap, R. K.; Zybko, W. C. Am. Dyest. Rep. 1968, 57,

1086.

(147) Allman, W. T. Am. Dyest. Rep. 1967, 56, 761.

(148) Montazer, M.; Sadighi, A. J. Appl. Polym. Sci. 2006, 100, 5049.

(149) Charpentier, P. A.; Maguire, A.; Wan, W. Appl. Surf. Sci. 2006, 252,

6360 .

(150) Walzak, M. J.; Flynn, S.; Foerch, R.; Hill, J. M.; Karbashewski, E.; Lin, A.; Strobel, M. J. Adhes. Sci. Technol. 1995, 9, 1229.

(151) Öktem, T.; Seventekin, N.; Ayhan, H.; Pişkin, E. Turk. J. Chem. 2000, 24, 275.

(152) Hossain, M. M.; Herrmann, A. S.; Hegemann, D. Plasma Process. Polym. 2006, 3, 299.

(153) Costa, T. H. C.; Feitor, M. C.; Alves-Jr., C.; Freire, P. B.; Bezerra, C. M. D. J. Mater. Process. Tech. 2006, 173, 40.

(154) Inagaki, N.; Narushim, K.; Tuchida, N.; Miyazaki, K. J. Polym. Sci. Pol. Phys. 2004, 42, 3727.

(155) De Geyter, N.; Morent, R.; Leys, C. Surf Coat Tech 2006, 201, 2460.

(156) Cireli, A.; Kutlu, B.; Mutlu, M. J. Appl. Polym. Sci. 2007, 104, 2318.

(157) De Geyter, N.; Morent, R.; Leys, C.; Gengembre, L.; Payen, E. Surf Coat Tech 2007, 201, 7066.

(158) Morent, R.; De Geyter, N.; Leys, C.; Gengembre, L.; Payen, E. Text. Res. J. 2007, 77, 471.

(159) Hyun, J.; Barletta, P.; Koh, K.; Yoo, S.; Oh, A.; Aspnes, D. E.; Cuom, J. J. J. Appl. Polym. Sci. 2000, 77, 1679.

(160) Riccardi, C.; Barni, R.; Selli, E.; Mazzone, G.; Massafra, M. R.; Marcandalli, B.; Poletti, G. Appl. Surf. Sci. 2003, 211, 386.

(161) Zhou, H.; Götzinger, M.; Peukertb, W. Powder Technol. 2003, 135, 82.

(162) Rabinovich, Y. I.; Adler, J. J.; Ata, A.; Singh, R. K.; Moudgil, B. M. J. Colloid Interf. Sci. 2000, 232, 10.

(163) Rabinovich, Y. I.; Adler, J. J.; Ata, A.; Singh, R. K.; Moudgil, B. M. J. Colloid Interf. Sci. 2000, 232, 17.

(164) Benz, M.; Rosenberg, K. J.; Kramer, E. J.; Israelachvili, J. N. J. Phys. Chem. B 2006, 110, 11884.

(165) Zhu, P. X.; Teranishi, M.; Xiang, J. H.; Masuda, Y.; Seo, W. S.; Koumoto, K. Thin Solid Films 2005, 473, 351.

(166) Athanasopoulos, D. C.; Garofalini, S. H. J. Chem. Phys. 1992, 97, 3775.

(167) Feuston, B. P.; Garofalini, S. H. J. Appl. Phys. 1990, 68, 4830.

(168) Garofalini, S. H. J. Chem. Phys. 1983, 78, 2069.

(169) Garofalini, S. H. Structure and Bonding in Non Crystaline Solid; Plenum Press: New York, 1985.

(170) Yarovsky, I.; Aguilar, M. I.; Hearn, M. T. W. Anal. Chem. 1995, 67, 2145.

(171) Yarovsky, I.; Aguilar, M. I.; Hearn, M. T. W. J. Phys. Chem. B 1997, $101,10962$.

(172) Zhuravlev, L. T. Langmuir 1987, 3, 316.

(173) Yang, R.; Rendell, A. P. J. Phys. Chem. B 2006, 110, 9608. 
(174) Rimai, D. S.; Alexandrovich, P.; Quesnel, D. J. J. Adhesion 2003, 79,

1041.

(175) Bhattacharjee, S.; Ko, C.-H.; Elimelech, M. Langmuir 1998, 14, 3365.

(176) Kuo, S.-W.; Wu, Y.-C.; Wang, C.-F.; Jeong, K.-U. J. Phys. Chem. C 2009, 113, 20666.

(177) Sun, T. L.; Wang, G. J.; Feng, L.; Liu, B. Q.; Ma, Y. M.; Jiang, L.; Zhu, D. B. Angew. Chem. Int. Edit. 2004, 43, 357.

(178) Lin, H. C.; Wang, C. F.; Kuo, S. W.; Tung, P. H.; Huang, C. F.; Lin, C. H.; Chang, F. C. J. Phys. Chem. B 2007, 111, 3404.

(179) Ma, K. X.; Chung, T. S. J. Phys. Chem. B 2001, 105, 4145.

(180) Hill, J. M.; Karbashewski, E.; Lin, A.; M., S.; Walzak, M. J. J. Adhes. Sci. Technol. 1995, 9, 1575.

(181) Peeling, J.; Courval, G.; Jazzar, M. S. J. Polym. Sci. Pol. Chem. 1984, 22, 419.

(182) Strobel, M.; Lyons, C. S.; Strobel, J. M.; Kapaun, R. S. J. Adhes. Sci. Technol. 1992, 6, 429.

(183) Jacobs, T.; Morent, R.; De Geyter, N.; Leys, C. Plasma Process. Polym. 2009, 6, S412.

(184) Steuerman, D. W.; Star, A.; Narizzano, R.; Choi, H.; Ries, R. S.; Nicolini, C.; Stoddart, J. F.; Heath, J. R. J. Phys. Chem. B 2002, 106, 3124.

(185) Gupta, B.; Hilborn, J.; Hollenstein, C.; Plummer, C. J. G.; Houriet, R.; Xanthopoulos, N. J. Appl. Polym. Sci. 2000, 78, 1083.

(186) Li, J. X.; Wang, J.; Shen, L. R.; Xu, Z. J.; Li, P.; Wan, G. J.; Huang, N. Surf Coat Tech 2007, 201, 8155.

(187) Sharma, P. K.; Rao, K. H. Adv. Colloid Interfac. 2002, 98, 341.

(188) Marmur, A. Biofouling 2006, 22, 107.

(189) Attard, P. Langmuir 2000, 16, 4455.

(190) Bers, A. V.; Wahl, M. Biofouling 2004, 20, 43.

(191) Scardino, A. J.; Harvey, E.; Nys, R. D. Biofouling 2006, 22, 55.

(192) Schumacher, J. F.; Carman, M. L.; Estes, T. G.; Feinberg, A. W.; Wilson, L. H.; Callow, M. E.; Callow, J. A.; Finlay, J. A.; Brennan, A. B. Biofouling 2007, $23,55$.

(193) Berendsen, H. J. C.; Postma, J. P. M.; vanGunsterenand, W. F.; Hermans, J. In Intermolecular Forces; Pullman, B., Ed.; D. Riedel: Dordrecht, 1981, p 331.

(194) Buck, M.; Karplus, M. J. Phys. Chem. B 2001, 105, 11000.

(195) Wang, J.; Kalinichev, A. G.; Kirkpatrick, R. J. Geochim. Cosmochim. Ac. 2004, 68, 3351 .

(196) Janecek, J.; Netz, R. R. Langmuir 2007, 23, 8417.

(197) Han, Q.; Urban, M. W. J. Appl. Polym. Sci. 2001, 81, 2045.

(198) Ghosh, S.; Krishnamurti, N. Polym.-Plast. Technol. 2001, 40, 539.

(199) Tretinnikov, O. N.; Ogata, S.; Ikada, Y. Polymer 1998, 39, 6115.

(200) Fisher, G. L.; Lakis, R. E.; Davis, C. C.; Szakal, C.; Swadener, J. G.; Wetteland, C. J.; Winograd, N. Appl. Surf. Sci. 2006, 253, 1330.

(201) Lukey, C. A.; Brown, H. R.; Spinks, G. M. J. Adhesion 2004, 80, 647.

(202) Theodorou, D. N.; Suter, U. W. Macromolecules 1986, 19, 139.

(203) Meirovitch, H. J. Chem. Phys. 1983, 79, 502. 
(204) Aldrighetti, C.; Tassone, P.; Ciardelli, F.; Ruggeri, G. Polym. Degrad. Stabil. 2005, 90, 346.

(205) Ni, H.; Daum, J. L.; Soucek, M. D.; Simonsick, W. J. J. Coating. Technol. 2002, 74, 49.

(206) Kaminski, A. M.; Urban, M. W. J. Coating. Technol. 1997, 69, 55.

(207) Oliver, W. C.; Pharr, G. M. J. Mater. Res. 1992, 7, 1564.

(208) Stevenson, M. E.; Bradt, R. C. Practical Fail. Anal. 2001, 5, 37.

(209) Miyake, K.; Satomi, N.; Sasaki, S. Appl. Phys. Lett. 2006, 89, 0319251.

(210) Podczeck, F. Particle-particle adhesion in pharmaceutical powder handling; Imperial College Press: London, UK, 1998.

(211) Butt, H. J.; Cappella, B.; Kappl, M. Surf. Sci. Rep. 2005, 59, 1.

(212) Rao, G. R.; Blau, P. J.; Lee, E. H. Wear 1995, 184, 213.

(213) Lukey, C. A.; Greenhalgh, A. K.; Brown, H. R. J. Adhesion 2007, 83, 319.

(214) Hasirci, N.; Aksoy, E. A. High. Perform. Polym. 2007, 19, 621.

(215) Kinning, D. J. J. Adhesion 2001, 75, 1.

(216) Fakirov, S.; Calleja, F. J. B.; Krumova, M. J. Polym. Sci. Pol. Phys. 1999, $37,1413$.

(217) Frings, S.; van Nostrum, C. F.; van der Linde, R.; Meinema, H. A.; Rentrop, C. H. A. J. Coating. Technol. 2000, 72, 83.

(218) Beake, B. D.; Leggett, G. J. Polymer 2002, 43, 319. 